\title{
Almost Disjoint Families em Topologia
}

\author{
Vinicius de Oliveira Rodrigues
}

DissERTAÇÃo APRESENTADA

$\mathrm{AO}$

Instituto DE MatemÁtica E EstatísticA

DA

UNIVERSIDADE DE SÃo PAUlo

PARA

OBTENÇÃO DO TÍTULO

$\mathrm{DE}$

Mestre em CiÊnCIAS

Programa: Mestrado em Matemática

Orientador: Prof. Dr. Artur Hideyuki Tomita

Durante o desenvolvimento deste trabalho o autor recebeu auxílio financeiro da FAPESP (Processo 2015/15166-7)

31 de julho de 2018 


\section{Almost Disjoint Families em Topologia}

Esta versão da dissertação/tese contém as correções e alterações sugeridas pela Comissão Julgadora durante a defesa da versão original do trabalho, realizada em 11/12/2017. Uma cópia da versão original está disponível no

Instituto de Matemática e Estatística da Universidade de São Paulo.

Comissão Julgadora:

- Prof. Dr. Artur Hideyuki Tomita (orientador) - IME-USP

- Prof ${ }^{\mathrm{a}}$. Dra ${ }^{\mathrm{a}}$. Ana Carolina Boero - UFABC

- Prof. Dr. Leandro Fiorini Aurichi - ICMC-USP 


\section{Agradecimentos}

À minha mãe Tânia e ao meu pai Alberto, sem o apoio de vocês, esta realização não teria sido possível,

Ao meu orientador Artur Hideyuki Tomita, por acreditar em mim desde o primeiro semestre da minha graduação e me apoiar ao longo de todo o caminho,

Aos meus colegas e também amigos de curso, pois sempre persistimos, estudamos e trabalhamos juntos,

À toda a minha família,

À FAPESP, que, por meio de apoio financeiro, tornou este projeto possível.

Obrigado a todos vocês. 


\section{Resumo}

Rodrigues, V. O. Almost Disjoint Families em Topologia. 2017. 126 f. Dissertação (Mestrado) - Instituto de Matemática e Estatística, Universidade de São Paulo, São Paulo, 2017.

Uma almost disjoint family é uma coleção infinita de subconjuntos infinitos de números naturais tal que a interseção de quaisquer dois de seus elementos distintos é finita. Almost disjoint families podem ser utilizadas para construir um espaço topológico associado chamado de $\Psi$-espaços, também conhecido como espaços de Mrówka. As propriedades topológicas deste espaço topológico dependem das propriedades combinatórias da família que o deu origem, e estes espaços podem ser utilizados para responder perguntas sobre topologia geral, muitas vezes não inicialmente relacionadas com almost disjoint families ou seus respectivos espaços de Mrówka. Neste documento, exploramos diversas construções envolvendo estes objetos utilizando combinatória infinita e princípios combinatórios como diamante, Axioma de Martin e técnicas como Forcing e tratamos de problemas envolvendo compactificações de Stone-Čech, espaços sequenciais, a propriedade de Lindelöf em espaços de funções, hiperespaços de Vietoris, dentre outros. O primeiro capítulo contém diversos pré-requisitos necessários para a leitura desta dissertação a fim de torná-la o mais autocontida possível. O segundo capítulo introduz as almost disjoint families e seus $\Psi$-espaços associados, provando diversas propriedades importantes. Os demais capítulos são independentes entre si e tratam de problemas de Topologia Geral que podem ser solucionados com estes conceitos, ou de problemas que derivam destes conceitos.

Palavras-chave: Topologia Geral, Almost Disjoint Families, Combinatória Infinita, Espaços de Mrówka. 


\section{Abstract}

Rodrigues, V. O. Almost Disjoint Families in Topology. 2017. 126 f. Dissertação (Mestrado) Instituto de Matemática e Estatística, Universidade de São Paulo, São Paulo, 2017.

An almost disjoint family is an infinite collection of infinite subsets of natural numbers such that the intersection of any two of its elements is finite. Almost disjoint families may be used to construct an associated topological space called psi space, also know as Mrówka space. The topological properties of this topological space depends on the combinatorical properties of the family that originated it, and these spaces may be used to answer questions in general topology, many times initially unrelated to almost disjoint families or to their Mrówka spaces. In this document, we explore several constructions involving these objects by using infinitary combinatorics and combinatorical principles like diamond, Martin's Axiom, forcing techniques and we treat abour problems regardins Stone-Čech compactifications, sequencial spaces, the property of Lindelöf on spaces of functions, hyperspaces of Vietoris, among others. The first chapter contains several pre requirements that are neccessary to read this dissertation in order to make it as self contained as possible. The second chapter introduces almost disjoint families and their associated Psi spaces, proving several important properties. The following chapters are independent from each other and treat about problems on General Topology that may be solved by using these concepts, or about problems that arises from these concepts.

Keywords: General Topology, Almost Disjoint Families, Infinitary Combinatorics, Mrówka Spaces. 


\section{Sumário}

1 Introdução $\quad \mathbf{1}$

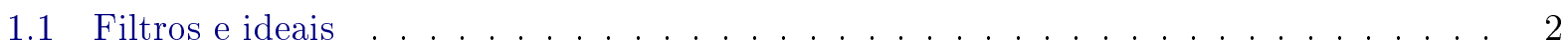

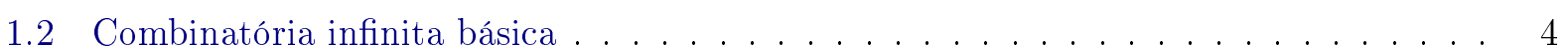

1.3 Axioma de Martin . . . . . . . . . . . . . . . . . . . . 5

1.4 Topologia da ordem e princípios combinatórios . . . . . . . . . . . 7

1.5 Espaços conexos, zero Dimensionais e totalmente desconexos . . . . . . . . . . . . 11

1.6 Compactificações . . . . . . . . . . . . . . . . . . . . 12

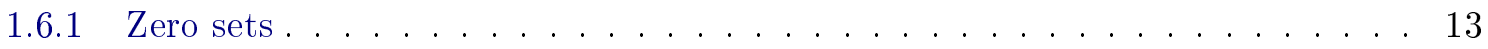

1.6.2 Compactificação por um ponto . . . . . . . . . . . . . . 15

1.6.3 Compactificação de Stone-Čech . . . . . . . . . . . . . . . . . . 16

1.7 Pseudocompacidade . . . . . . . . . . . . . . . . . . . . 20

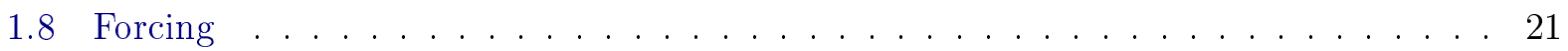

1.8.1 Adicionando uma Interpretação $M \ldots \ldots \ldots \ldots \ldots$. . . . . . . 22

1.8 .2 Forcing . . . . . . . . . . . . . . . . . . 23

2 Almost disjoint families e $\Psi$-espaços $\quad 27$

2.1 Almost disjoint families . . . . . . . . . . . . . . . 27

2.2 Pequenos Cardinais . . . . . . . . . . . . . . . . . . . . . . . . . 29

$2.3 \quad \Psi$-espaços . . . . . . . . . . . . . . . . . . . . . . 33

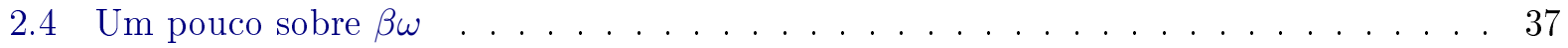

2.5 Almost distoint families e happy families . . . . . . . . . . . . . . . . 40

2.6 Normalidade . . . . . . . . . . . . . . . . . . . . . . . . . . 42

3 Espaços de funções $\quad 45$

3.1 A propriedade de Lindelöf em um espaço de funções . . . . . . . . . . . . . . . . . 45

$4 \quad \Psi$-espaços e espaços sequenciais $\quad 55$

4.1 Introdução . . . . . . . . . . . . . . . . . . . . . . . 55

4.2 Espaços sequenciais e espaços de Fréchet . . . . . . . . . . . . . . . . 55

5 Os espaços $\beta(\Psi(\mathcal{A})) \quad \mathbf{6 1}$

5.1 Compactificação por dois pontos . . . . . . . . . . . . . . 61

5.2 Unicidade de compactificação . . . . . . . . . . . . . . . . . . . . . . 64

5.3 Mais sobre famílias de Mrówka . . . . . . . . . . . . . . . . . . 66

5.4 Espaços métricos compactos e remainders de $\Psi$-espaços . . . . . . . . . . . . . . . 70 
6 MAD families e os racionais $\quad 73$

6.1 Introdução . . . . . . . . . . . . . . . . . . . . 73

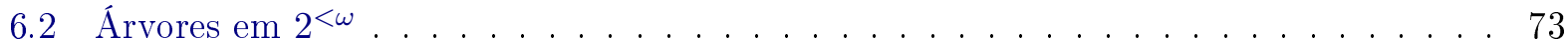

6.3 O Forcing de Sacks . . . . . . . . . . . . . . . . . 78

7 Pseudocompacidade de hiperespaços $\quad 83$

7.1 Introdução . . . . . . . . . . . . . . . . . . . . . . . . . . 83

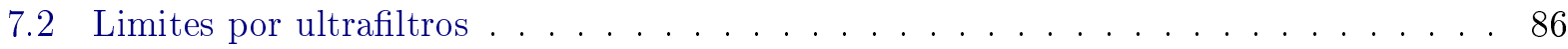

7.3 Pseudocompacidade de $\mathrm{CL}(\Psi(\mathcal{A}))$ sob $\mathfrak{p}=\mathfrak{c} \ldots \ldots \ldots \ldots$. . . . . . . 88

7.4 O Base Tree Lemma . . . . . . . . . . . . . . . . . . . . . . . 91

7.5 Pseudocompacidade de $\mathrm{CL}(\Psi(\mathcal{A}))$ sob $\mathfrak{h}<\mathfrak{c} \ldots \ldots \ldots \ldots \ldots$

$7.6 \mathrm{Um}$ exemplo em ZFC . . . . . . . . . . . . . . . . . . . . . 97

$\begin{array}{ll}\text { A MA } \sigma \text {-Centrado } & 101\end{array}$

B O Forcing de Cohen e pequenos cardinais $\quad 105$

B.1 O modelo de Cohen . . . . . . . . . . . . . . . . . . . . . . . . . . 105

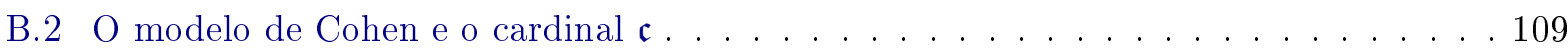

B.3 O modelo de Cohen e o cardinal $\mathfrak{b} \ldots \ldots \ldots \ldots \ldots$. . . . . . . . . . . . . . . . . . . . . .

B.4 O Modelo de Cohen e o cardinal $\mathfrak{a} \ldots \ldots \ldots \ldots$. . . . . . . . . . . . . . . . .

B.5 O Modelo de Cohen e o cardinal d . . . . . . . . . . . . . . . . 112

Referências Bibliográficas $\quad 115$ 


\section{Capítulo 1}

\section{Introdução}

Uma almost disjoint family, que é uma generalização natural de família disjunta, é uma coleção infinita de subconjuntos infinitos de números naturais tal que a interseção de quaisquer dois de seus elementos distintos é finita. Almost disjoint families podem ser utilizadas para construir um espaço topológico associado chamado de $\Psi$-espaços, também conhecido como espaços de Mrówka. Conforme exemplificaremos diversas vezes, as propriedades topológicas de $\Psi$-espaços dependem das propriedades combinatórias da almost disjoint family que os deu origem, e estes espaços podem ser utilizados para responder perguntas sobre topologia geral, muitas vezes não inicialmente relacionadas com almost disjoint families ou seus respectivos espaços de Mrówka. Tais espaços são muito úteis na construção de exemplos que respondem perguntas em Topologia Geral que nem sempre são relacionadas inicialmente a eles ou a almost disjoint families, como ficará evidenciado neste documento. Além disso, existe interesse em estudar puramente tanto almost disjoint families como seus $\Psi$-espaços associados.

Neste primeiro capítulo, revisaremos algumas noções e definições essenciais para compreender o material desta dissertação. Para este fim, daremos as definições e provaremos alguns fatos mais básicos sobre topologia geral com a finalidade de tornar esse documento o mais autocontido possível. Nos basearemos principalmente nos livros [Kun80], [Kun11] e [Wal75].

No Capítulo 2, definiremos almost disjoint families, $\Psi$-espaços e apresentaremos alguns teoremas sobre estes objetos que podem ser encontrados principalmente em [Kun80], [Kun11], [Bla10], [Mro70], [Mro77], [Hru14] e [Wal75] (para a seção sobre $\beta \omega$ ) e [Mat77] (para a seção sobre happy families).

No Capítulo 3, estudaremos resultados sobre a propriedade de Lindelöf em espaços de funções a luz de $\Psi$-espaços, seguindo primariamente o artigo [DS06].

No Capítulo 4, trataremos de espaços sequenciais e de Fréchet, exibindo, utilizando MAD families, um produto de dois espaços compactos Hausdorff de Fréchet cujo produto não é de Fréchet, originalmente de [Sim80]. Nos basearemos principalmente nos artigos [Fra65], [Fra67] e [Dow95].

No Capítulo 5, estudaremos compactificações de Stone-Čech de espaços de Mrówka, baseandonos principalmente em construções dos artigos [Hru14], [Mro70], [Mro77] e [Ter80]. Veremos uma condição necessária e suficiente para que o remainder seja conexo, estudaremos o problema da unicidade de compactificação e mostraremos que todo espaço métrico compacto sem pontos isolados é o remainder do $\Psi$-espaço de alguma mad family.

O Capítulo 6, baseado em [Hru01], trata da absolutidade do conceito de MAD family com relação a forcing, enfatizando o Forcing de Sacks. Em particular, mostraremos que existe uma MAD family Sacks-indestrutível.

O Capítulo 7 estuda a pseudocompacidade de hiperespaços de Vietoris utilizando espaços de Mrówka. Baseamo-nos nos artigos [Mic51] e [HHR07]. Neste capítulo, mostramos também uma nova demonstração, que consideramos mais simples, para o fato de que $\mathfrak{t}=\mathfrak{c}$ implica que todo hiperespaço de Vietoris de uma família de Mrówka é pseudocompacto.

No Apêndice A, como material complementar, mostramos que $\mathfrak{p}$ é o primeiro cardinal $\kappa$ para o qual o qual $\mathrm{MA}_{\sigma}(\kappa)$ falha. No Apêndice $\mathrm{B}$, discutimos sobre o comportamento de alguns dos 
pequenos cardinais no modelo de Cohen, provando a consistência de algumas desigualdades dentre eles. Para os apêndices, nos baseamos primariamente em [Kun11].

\subsection{Filtros e ideais}

Começaremos com definições e fatos básicos sobre filtros e ideais.

Definição 1.1. Um ideal $\mathcal{I}$ em $X$ é uma coleção não vazia de subconjuntos de $X$ satisfazendo:

a) Para todos $A, B \subseteq X$, se $A \in \mathcal{I}$ e $B \subseteq A$ então $B \in \mathcal{I}$.

b) Se $A, B \in \mathcal{I}$ então $A \cup B \in \mathcal{I}$

Se $\mathcal{I}$ é um ideal tal que $X \notin \mathcal{I}$, então $\mathcal{I}$ é dito ser um ideal próprio.

Se $\mathcal{I}$ é um ideal próprio para o qual não existe um ideal próprio que contém $\mathcal{I}$ propriamente, dizemos que $\mathcal{I}$ é um ideal primo (ou maximal).

Um filtro $\mathcal{F}$ em $X$ é uma coleção não vazia de subconjuntos de $X$ satisfazendo:

a) Para todos $A, B \subseteq X$, se $A \in \mathcal{F}$ e $A \subseteq B$ então $B \in \mathcal{F}$.

b) Se $A, B \in \mathcal{F}$ então $A \cap B \in \mathcal{F}$

Se $\mathcal{F}$ é um filtro tal que $\emptyset \notin \mathcal{I}$, então $\mathcal{F}$ é dito ser um filtro próprio.

Se $\mathcal{F}$ é um filtro próprio para o qual não existe um filtro próprio que contém $\mathcal{F}$ propriamente, dizemos que $\mathcal{F}$ é um ultrafiltro.

O filtro dual de um ideal $\mathcal{I}$ em $X$, é o conjunto $\mathcal{I}^{*}=\{X \backslash A: A \in \mathcal{I}\}$.

O ideal dual de um filtro $\mathcal{I}$ em $X$, é o conjunto $\mathcal{F}^{*}=\{X \backslash A: A \in \mathcal{F}\}$.

Seja $\kappa$ um cardinal. Um filtro (ideal) é dito $\kappa$-completo se for fechado para interseções (uniões) de cardinalidade $<\kappa$.

Filtros podem ser pensados como uma coleção de subconjuntos "grandes" de $X$ (como, por exemplo, os conjuntos cofinitos, coenumeráveis, etc), e ideais, subconjuntos "pequenos" (como os finitos, os enumeráveis, etc). Na literatura, é comum encontrar textos que exigem que para que $\mathcal{F}$ seja filtro, que $\emptyset \notin \mathcal{F}$, e que para que $\mathcal{I}$ seja ideal, que $X \notin \mathcal{I}$. Definindo-se filtro e ideal dessa forma, é claro que o conceito de ideal próprio e de filtro próprio torna-se redundante. Nesse texto, preferimos tomar a abordagem da definição acima, admitindo que $\mathscr{P}(X)$ seja um ideal (filtro) não próprio.

A proposição abaixo decorre imediatamente das definições e pode facilmente ser verificada pelo leitor.

Proposição 1.1. Sejam $\mathcal{I}, \mathcal{F}$ um ideal e um filtro em $X$, respectivamente. Então $\mathcal{I}^{*}$ é um filtro, $\mathcal{F}^{*}$ é um ideal e valem as igualdades $\left(\mathcal{I}^{*}\right)^{*}=I,\left(\mathcal{F}^{*}\right)^{*}=F$. A propriedade de ser próprio é preservada por essa operação. Além disso, o ideal dual de um ultrafiltro é um ideal primo, e o filtro dual de um ideal primo é um ultrafiltro.

Note que o conjunto de todos os subconjuntos finitos de $X \neq \emptyset$, denotado por $[X]^{<\omega}$ é um ideal próprio em $X$. O seu dual é o filtro dos subconjuntos cofinitos de $X$, que, no caso de $X=\omega$, denotaremos por Fr, e este é chamado de Filtro de Fréchet.

Notemos que a união de uma cadeia de filtros (ideais) próprios em $X$ também é um filtro (ideal) próprio em $X$. Portanto, a proposição abaixo é uma aplicação imediata do lema de Zorn e a demonstração fica a cargo do leitor.

Proposição 1.2. Todo filtro próprio está contido em um ultrafiltro e todo ideal próprio está contido em um ideal primo. 
Definição 1.2. Seja $X$ um conjunto não vazio. Dizemos que $\mathcal{A} \subseteq \mathscr{P}(X)$ tem a propriedade da interseção finita (pif) se para todo $F \subseteq \mathcal{A}$ não vazio e finito, $\cap F \neq \emptyset$.

Note que todo filtro próprio tem pif. Para uma recíproca parcial, veremos abaixo que toda coleção de subconjuntos de $X$ que possui a pif está contida em ao menos um filtro próprio.

Proposição 1.3. Seja $X$ um conjunto não vazio e $\mathcal{A} \subseteq \mathscr{P}(X)$. Existe um filtro próprio $\mathcal{F}$ que contém $\mathcal{A}$ se, e somente se, $\mathcal{A}$ tem pif. Nesse caso, existe um filtro próprio $\mathcal{F}$ com a propriedade de ser menor filtro que contém $\mathcal{A}$, ou seja, se $\mathcal{G}$ é um filtro que contém $\mathcal{A}$, então $\mathcal{G}$ contém $\mathcal{F}$.

Demonstração. Suponha que $\mathcal{A}$ tem pif. Seja $\mathcal{F}=\{C \subseteq X: \exists B \subseteq \mathcal{A}$ (B é finito, não vazio e $\cap B \subseteq$ $C)\} \cup\{X\}$. ${ }^{1}$ Temos que $\mathcal{F}$ é não vazio. Se $C \in \mathcal{F}$ e $C \subseteq D \subseteq X$, temos que ou $X=C=D$, e, nesse caso, $D \in \mathcal{F}$, ou existe $B \subseteq X$ não vazio e finito tal que $\bigcap B \subseteq C \subseteq D$, e, portanto, $D \in \mathcal{F}$. Se $C, D \in \mathcal{F}$ são distintos de $X$, existem $A, B \subseteq X$ finitos e não vazios tais que $\bigcap A \subseteq C, \bigcap B \subseteq D$. Temos que $\bigcap(A \cup B) \subseteq C \cap D$, logo, $C \cap D \in \mathcal{F}$. Caso um deles seja $X$, a interseção entre eles é igual ao outro. Finalmente, $\emptyset \notin \mathcal{F}$ pois, caso contrário teríamos que $X=\emptyset$ ou existiria $B \subseteq \mathcal{A}$ finito tal que $\bigcap B=\emptyset$, o que viola a pif. Assim, $\mathcal{F}$ é um filtro próprio.

Temos que $\mathcal{A} \subseteq \mathcal{F}$, pois, dado $C \in \mathcal{A}$, basta tomar $B=\{C\}$. Resta ver que $\mathcal{F}$ é o menor filtro que contém $\mathcal{A}$. Suponha que $\mathcal{G}$ é filtro e que $\mathcal{A} \subseteq \mathcal{G}$. Fixe $C \in \mathcal{F}$. Existe $B \subseteq \mathcal{A}$ finito e não vazio tal que $\cap B \subseteq C$. Como $\mathcal{G}$ é filtro e $B \subseteq \mathcal{G}$ é finito e não vazio, temos que $\cap B \in \mathcal{G}$. Como $\cap B \in \mathcal{G}$ e $\cap B \subseteq C$, temos que $C \in \mathcal{G}$. Logo, $\mathcal{F} \subseteq \mathcal{G}$.

Reciprocamente, se existe um filtro próprio $\mathcal{F}$ que contém $\mathcal{A}$, todo subconjunto finito de $\mathcal{A}$ não vazio tem interseção não vazia, caso contrário teríamos que $\emptyset \in \mathcal{F}$.

Reunindo as proposições 1.3 e 1.2, obtemos o seguinte corolário:

Corolário 1.4. Seja $X \neq \emptyset$ um conjunto. Se $\mathcal{A} \subseteq \mathscr{P}(X)$ possui a pif, então existe $\mathcal{F}$ ultrafiltro tal que $\mathcal{A} \subseteq \mathcal{F}$.

Definição 1.3. Seja $\mathcal{A}$ uma coleção de conjuntos de $X \neq \emptyset$. O menor filtro que contém $\mathcal{A}$ é denotado por $\langle\mathcal{A}\rangle$ e chamado de filtro (em $X$ ) gerado por $\mathcal{A}$. Note que, caso $\mathcal{A}$ não tenha pif, temos que $\langle\mathcal{A}\rangle=\mathscr{P}(X)$, e, pela construção da Proposição 1.3 , se $\mathcal{A}$ tem pif, então $\langle\mathcal{A}\rangle$ é próprio.

A proposição abaixo é muito útil ao se trabalhar com a noção de ultrafiltro, e, em diversos textos, é tomada como definição de ultrafiltro.

Proposição 1.5. Um filtro próprio $\mathcal{F}$ em $X$ é um ultrafiltro se, e somente se, para todo $A \subseteq X$, ou $A \in \mathcal{F}$ ou $X \backslash A \in \mathcal{F}$.

Demonstração. Suponha que $\mathcal{F}$ é um ultrafiltro e fixe $A \subseteq X$. Suponha que $X \backslash A \notin \mathcal{F}$. Veremos que $A \in \mathcal{F}$. Suponha por absurdo que não. Veremos que $\mathcal{F} \cup\{A\}$ tem pif, e, portanto, está contido em um filtro, o que é absurdo. Seja $\mathcal{A} \subseteq \mathcal{F}$ finito e não vazio. Suponha por absurdo que $A \cap \bigcap \mathcal{A}=\emptyset$. Então $\bigcap \mathcal{A} \subseteq X \backslash A, \operatorname{logo}, X \backslash A \in \mathcal{F}$, absurdo. Logo, $\mathcal{F} \cup\{A\}$ tem pif, e, assim, $\mathcal{F}$ não é ultrafiltro.

Reciprocamente, suponha que $\mathcal{F}$ seja um filtro próprio tal que para $A \subseteq F$, ou $A \in \mathcal{F}$ ou $X \backslash A \in \mathcal{F}$. Suponha que $\mathcal{G} \supset \mathcal{F}$ seja um filtro próprio. Veremos que vale a inclusão contrária. Fixe $A \in \mathcal{G}$. Temos que $A \in \mathcal{F}$ ou $X \backslash A \in \mathcal{F}$. Se ocorrer a segunda opção, temos que $X \backslash A \in \mathcal{G}$ e $A \in \mathcal{G}$, logo, $\emptyset \in \mathcal{G}$, absurdo. Assim, $A \in \mathcal{F}$.

Trabalhando com a dualidade entre filtros e ideias, obtemos o seguinte corolário:

Corolário 1.6. Um ideal próprio $\mathcal{I}$ em $X$ é um ideal primo se, e somente se, para todo $A \subseteq X$, ou $A \in \mathcal{I}$ ou $X \backslash A \in \mathcal{I}$.

Notemos que dado $x \in X,\langle\{x\}\rangle=\{A \subseteq X: x \in A\}$ é um ultrafiltro.

\footnotetext{
${ }^{1} \mathrm{~A}$ união com $\{X\}$ só é necessária para incluir o caso $\mathcal{A}=\emptyset$.
} 
Definição 1.4. Um ultrafiltro é dito fixo ou principal se ele for do tipo $\langle\{x\}\rangle$ para algum $x \in X$. Um filtro é dito livre se ele contém todos os subconjuntos cofinitos de $X$. Um ideal em $X$ é dito livre se contém todos os subconjuntos finitos de $X$.

A proposição abaixo diz que todo ultrafiltro ou é fixo, ou é livre.

Proposição 1.7. Um ultrafiltro em $X$ é fixo se, e somente se, ele não é livre.

Demonstração. Mostraremos que se $\mathcal{F}$ é um ultrafiltro em $X$ que possui um elemento finito, então $X$ é fixo. Suponha que $\mathcal{F}$ é um filtro que possui um elemento finito. Seja $A \in \mathcal{F}$ um conjunto de menor cardinalidade possível. Sabemos que $A$ é finito e não vazio, assim, fixe $x \in \mathcal{A}$ qualquer. Temos que $\{x\} \in \mathcal{F}$ ou $X \backslash\{x\} \in \mathcal{F}$. Se a segunda opção ocorrer, $(X \backslash\{x\}) \cap A=A \backslash\{x\} \in \mathcal{F}$, o que é absurdo pois $|A \backslash\{x\}|<|A|$. Logo, $\{x\} \in \mathcal{F}$. Assim, $\langle\{x\}\rangle \subseteq \mathcal{F}$. Como o primeiro é ultrafiltro, segue a igualdade.

Reciprocamente, se $X$ é fixo, segue diretamente da definição que ele possui um conjunto unitário como elemento, assim, ele não é livre.

A definição seguinte será particularmente útil ao se falar em happy families.

Definição 1.5. Um ideal $\mathcal{I}$ em $X$ é dito alto se para todo $Y \subseteq X$ infinito existe $I \in \mathcal{I}$ tal que $I \cap Y$ é infinito.

Dado um ideal $\mathcal{I}$ em $X$, definimos $\mathcal{I}^{+}=\mathscr{P}(X) \backslash \mathcal{I}$, e um elemento deste conjunto é dito ser $\mathcal{I}$-positivo. Se $Y$ é $\mathcal{I}$-positivo, denotamos por $Y \mid \mathcal{I}$ o ideal em $Y$ dado por $\{Y \cap I: I \in \mathcal{I}\}$.

Como um exemplo, notemos que se $\mathcal{I}$ é o ideal dos conjuntos finitos em $X$, então $\mathcal{I}^{+}$é a coleção dos subconjuntos infinitos de $X$.

Por fim, apenas inseriremos uma nomenclatura para falar do conjunto dos ultrafiltros livres sobre $\omega$, que, mais tarde, veremos ser a compactificação de Stone-Čech de $\omega$.

Definição 1.6. $\omega^{*}$ é o conjunto de todos os ultrafiltros livres de $\omega$.

\subsection{Combinatória infinita básica}

Nessa seção, explicitaremos as demonstrações de algumas proposições simples sobre combinatória infinita básica que usaremos ao longo da dissertação.

Lema 1.8. Sejam $A, B$ conjuntos infinitos de cardinalidade $\kappa$. Então existem $2^{\kappa}$ funções bijetoras de $A$ em $B$.

Demonstração. Seja $F$ o conjunto de todas as funções bijetoras de $A$ em $B$. Temos que $|F| \leq$ $\left|A^{B}\right|=\kappa^{\kappa}=2^{\kappa}$. Para a inclusão contrária, particionemos $A$ em dois conjuntos de cardinalidade $\kappa$, denotados por $C, D$. Fixe $\phi: A \rightarrow B$ bijetora. Para cada $K \subseteq C$, fixe $f_{K} \in F$ tal que $f_{K}(x)=\phi(x)$ para todo $x \in K$, e $f_{K}(x) \in \phi[D]$ para todo $x \in C \backslash K$. Se $K, L \subseteq A$, temos que se $K \neq L$ então $f_{K} \neq f_{L}$. Como $|\mathscr{P}(A)|=2^{\kappa}$, segue a tese.

Definição 1.7. Uma função $n$-ária em $A$ é uma função $f: A^{n} \rightarrow A$. Se $B \subseteq A$, dizemos que $B$ é fechado sobre $f$ se $f^{n}[B] \subseteq B$. Uma função finitária é uma função $n$-ária para algum $n$ natural. Se $F$ é uma coleção de funções finitárias em $A$, o fecho de $B$ por $F$ é a interseção de todos os subconjuntos de $A$ fechados por cada $f \in F$, o que coincide com o menor subconjunto de $A$ fechado por cada função de $F$ (que é a interseção de todos os subconjuntos de $A$ fechados por cada $f \in F$ ).

Proposição 1.9. Seja $\kappa$ um cardinal infinito e $A$ um conjunto. Suponha que $B \subseteq A, B \leq \kappa$ e que $F$ é um conjunto de $\leq \kappa$ funções finitária em $A$. Então o fecho de $B$ por $F$ tem cardinalidade $\leq \kappa$. 
Demonstração. Se $D \subseteq A$ e $f \in F$ é $n$-ária, seja $f * D=f\left[D^{n}\right]$. Note que se $|D| \leq \kappa$ então $|f * D| \leq \kappa$. Seja $C_{0}=B$. Definido $C_{n}$, seja $C_{n+1}=C_{n} \cup \bigcup\left\{f * C_{n}: f \in F\right\}$. Temos que cada $C_{n}$ tem cardinalidade $\leq \kappa$. Seja $C_{\omega}=\bigcup_{n \in \omega} C_{n}$. Temos que $C_{\omega}$ tem cardinalidade $\leq \kappa$ e é fechado por $F$.

Lema 1.10 (Lema de König). Suponha que $\kappa, \lambda$ são cardinais infinitos e que $\operatorname{cf} \kappa \leq \lambda$. Então $\kappa^{\lambda}>\kappa$. Além disso, para todo cardinal $\theta, \operatorname{cf} 2^{\theta}>\theta$

Demonstração. Para a primeira afirmação, seja $f: \lambda \rightarrow \kappa$ cofinal. Seja $g: \kappa \rightarrow \kappa^{\lambda}$. Veremos que $g$ não é sobrejetora. Seja $h: \lambda \rightarrow \kappa$ dada por:

$$
h(\alpha)=\min (\kappa \backslash\{(g(\mu))(\alpha): \mu<f(\alpha)\}) .
$$

Afirmo que $h \notin \operatorname{ran} g$. Pois suponha que esteja. Existe $\mu$ tal que $g\left(\mu_{0}\right)=h$. Como $f$ é cofinal, existe $\alpha$ tal que $f(\alpha)>\mu_{0}$. Assim, $h(\alpha) \in(\kappa \backslash\{(g(\mu))(\alpha): \mu<f(\alpha)\})$, o que é absurdo pois $\mu_{0}<f(\alpha)$ e $\left(g\left(\mu_{0}\right)\right)(\alpha)=h(\alpha)$.

Para a segunda afirmação, suponha por absurdo que $\operatorname{cf} 2^{\lambda} \leq \lambda$. Então, pela primeira afirmação, $\left(2^{\lambda}\right)^{\lambda}>2^{\lambda}$, o que é absurdo.

Proposição 1.11. Suponha GCH, a hipótese generalizada do contínuo (i.e, $2^{\kappa}=\kappa^{+}$para todo cardinal $\kappa$ ). Sejam $\lambda \geq 1$ um cardinal e $\kappa$ um cardinal infinito. Então:

i) $\kappa \leq \lambda \rightarrow \kappa^{\lambda}=\lambda^{+}$.

ii) $\kappa>\lambda \geq \operatorname{cf} \kappa \rightarrow \kappa^{\lambda}=\kappa^{+}$.

iii) $\lambda<\operatorname{cf} \kappa \rightarrow \kappa^{\lambda}=\kappa$.

Demonstração. Provaremos cada item, em ordem.

i) Temos que $\lambda^{+}=2^{\lambda} \leq \kappa^{\lambda} \leq \lambda^{\lambda} \leq\left(2^{\lambda}\right)^{\lambda}=2^{\lambda}=\lambda^{+}$, logo, valem todas as igualdades intermediárias.

ii) Pelo Lema de König, $\kappa^{\lambda}>\kappa$, logo, $\kappa^{+} \leq \kappa^{\lambda} \leq \kappa^{\kappa}=2^{\kappa}=\kappa^{+}$, assim, segue a tese.

iii) Primeiro, notemos que a hipótese implica que ${ }^{\lambda} \kappa=\bigcup\left\{{ }^{\lambda} \alpha: \alpha<\kappa\right\}$. Logo, $\kappa^{\lambda} \leq \sup \left\{|\alpha|^{\lambda}\right.$ : $\alpha<\kappa\}$.. . Dado $\alpha<\kappa$, se $|\alpha| \leq \lambda$, de i) segue que $|\alpha|^{\lambda}=\lambda^{+} \leq \mathrm{cf} \kappa \leq \kappa$. Se $\lambda \leq|\alpha|$, temos que $|\alpha|^{\lambda} \leq|\alpha|^{|\alpha|}=2^{|\alpha|}=|\alpha|^{+} \leq \kappa$. Assim, segue a tese.

\subsection{Axioma de Martin}

Definição 1.8. Uma pré-ordem é um par $(P, \leq)$, onde $\leq$ é uma relação reflexiva e transitiva em $P$, ou seja:

a) Para todo $a \in P, a \leq a$ (propriedade reflexiva).

b) Para todos $a, b, c \in P$, se $a \leq b$ e $B \leq c$ então $a \leq c$ (propriedade transitiva).

Notemos que não estamos exigindo a propriedade antissimétrica (para todos $a, b \in P$, se $a \leq b$ e $b \leq a$, então $a=b)$. Caso $(P, \leq)$ seja uma pré-ordem que satisfaça essa última propriedade, ela é dita uma ordem parcial.

Dizemos que $F \subseteq P$ é um filtro se for não vazio e satisfizer as seguintes propriedades:

a) Para todos $a, b \in P$, se $a \leq b$ e $a \in F$ então $b \in F$ ( $F$ é fechado para cima).

b) Para todos $a, b \in F$ existe $c \in F$ tal que $c \leq a$ e $c \leq b$. 
Se $a, b \in P$ são tais que existe $c \in P$ tal que $c \leq a$ e $c \leq b$, então $a, b$ são ditos compatíveis. Caso contrário, eles são ditos incompatíveis e escreve-se $a \perp b$.

Uma anticadeia em $(P, \leq)$ é um subconjunto $A \subseteq P$ tal que para todos $a, b \in \mathcal{A}$ temos que $a \perp b$. Dizemos que $(P, \leq)$ tem a condição de anticadeia enumerável (countable antichain condition, c.c.c.) se toda anticadeia em $(P, \leq)$ é enumerável.

$\mathrm{MA}(\kappa)$ é a seguinte afirmação: Para toda pré-ordem $(P, \leq)$ com c.c.c. e para qualquer coleção $\mathcal{C}$ de subconjuntos densos de $P \operatorname{com}|\mathcal{C}| \leq \kappa$, existe um filtro $\mathcal{F}$ tal que $F \cap D$ para todo $D \in \mathcal{C}$.

$\mathfrak{m}$ é o menor cardinal $\kappa$ para o qual vale $\neg \mathrm{MA}(\kappa)$.

$\mathrm{O}$ axioma de Martin é a afirmação $\mathfrak{m}=\mathfrak{c}$. Veremos a seguir que em ZFC podemos provar que $\omega<\mathfrak{m} \leq \mathfrak{c}$.

Se $P$ é uma pré-ordem, $\operatorname{MA}_{P}(\kappa)$ é a seguinte afirmação: para qualquer coleção $\mathcal{C}$ de subconjuntos densos de $P$ tal que $|\mathcal{C}| \leq \kappa$, existe um filtro $\mathcal{F}$ em $P$ tal que $F \cap D$ para todo $D \in \mathcal{C}$.

Proposição 1.12. $\omega<\mathfrak{m} \leq \mathfrak{c}$.

Demonstração. Para mostrar que vale $M A(\omega)$, fixe uma pré-ordem $P$ com c.c.c. e seja $\left\{D_{n}: n \in \omega\right\}$ uma coleção de subconjuntos densos em $P$. Fixe $x_{0} \in D_{0}$. Definido $x_{n}$, tome $x_{n+1} \in D_{n+1}$ com $x_{n+1} \leq x_{n}$. Por fim, considere $F=\left\{p \in P: \exists n \in \omega\left(x_{n} \leq p\right)\right\}$. Fica a cargo do leitor verificar que $F$ é um filtro, e temos que dado $n, x_{n} \in F \cap D_{n}$.

Para mostrar que não vale $M A(\mathfrak{c})$, suponha por absurdo que vale. Seja $P$ o conjunto das funções parciais finitas de $\omega$ em 2 , com a ordem da inclusão reversa, ou seja,

$$
P=\bigcup_{A \in[\omega]<\omega} 2^{A}
$$

de modo que $p \leq q \leftrightarrow q \subseteq p$.

Para cada $f \in \omega^{\omega}$, seja $D_{f}=\{p \in P: p \nsubseteq f\}$. Para cada $n \in \omega$, seja $E_{n}=\{p \in P: n \in \operatorname{dom} p\}$. Note que $P$ tem c.c.c., pois é enumerável. Por hipótese, existe um filtro $F$ que intersecta cada $E_{n}$ e cada $D_{f}$. Temos que $g=\bigcup F$ é uma função, pois é uma união de funções compatíveis. Temos que o domínio de $g$ é $\omega$, pois dado $n$ natural, existe $p \in F \cap D_{n}$. Assim, $g: \omega \rightarrow 2$. Porém, tomando $p \in F \cap D_{g}$, segue que $p \nsubseteq g$, o que é absurdo.

Observação: Notemos que para provar $\mathrm{MA}(\omega)$, não foi necessário utilizar que $P$ possui c.c.c. Assim, temos que para toda pré-ordem $P$, vale $\operatorname{MA}_{P}(\omega)$.

Por fim, provaremos o seguinte lema envolvendo o cardinal $\mathfrak{m}$, que será utilizado na seção sobre pequenos cardinais para provar algumas implicações do Axioma de Martin e desigualdades envolvendo o cardinal $\mathfrak{m}$.

Proposição 1.13. $\kappa<\mathfrak{m}$ implica que para todo $\mathcal{A}, \mathcal{C} \subseteq \mathscr{P}(\omega)$ tais que $|\mathcal{A}|,|\mathcal{C}|<\kappa$ e tais que para todo $y \in \mathcal{C}$ e para todo $F \subseteq \mathcal{A}$ finito, $|y \backslash \bigcup F|=\omega$, existe $D \subseteq \omega$ tal que $\forall x \in \mathcal{A}(|D \cap x|<\omega)$ e $\forall Y \in \mathcal{C}(|D \cap Y|=\omega)$.

Demonstração. Considere $P=[\omega]^{<\omega} \times[\mathcal{A}]^{<\omega}$ ordenado por:

$$
\left(s^{\prime}, F^{\prime}\right) \leq(s, F) \leftrightarrow s \subseteq s^{\prime} \wedge F \subseteq F^{\prime} \wedge \forall X \in F\left(X \cap s^{\prime} \subseteq s\right)
$$

É fácil verificar que $\leq$ é uma pré-ordem e que para todo $s \in[\omega]^{<\omega}$ e para todos $F, F^{\prime} \in[\mathcal{A}]^{<\omega}$, $\left(s, F \cup F^{\prime}\right)$ é uma extensão comum de $(s, F)$ e $\left(s, F^{\prime}\right)$. Isso mostra que se $\mathcal{B}$ é uma anticadeia de $P$ e $(s, F) \neq\left(s^{\prime}, F^{\prime}\right)$ são elementos de $\mathcal{B}$, então $s \neq s^{\prime}$. Como $\left.[\omega]\right]^{<\omega}$ é enumerável, segue que a anticadeia é enumerável. Assim, $P$ tem c.c.c.

Para cada $X \in \mathcal{A}$, seja $E_{X}=\{(s, F): X \in F\}$. Temos que $E_{X}$ é denso pois dado $(s, F)$, temos que $(s, F \cup\{X\}) \leq(s, F)$.

Para cada $n \in \omega$ e $Y \in \mathcal{C}$, seja $D_{n}^{Y}=\{(s, F) \in P: s \cap Y \nsubseteq n\}$.

Temos que cada $D_{n}^{Y}$ é denso: dado $(s, F)$, tomemos $m \in Y \backslash \bigcup F$ com $m \geq n$ e considere $(s \cup\{m\}, F)$. 
Seja $G$ filtro intersectando cada um $\operatorname{dos} E_{X}, D_{n}^{Y}$. Seja $D=\bigcup\{s:(s, F) \in F\}$. Dado $X \in \mathcal{A}$, seja $(s, F) \in G \operatorname{com} X \in F$. Afirmo que $X \cap D \subseteq s$. Com efeito, dado $\left(s^{\prime}, F^{\prime}\right) \in G$, temos que existe $\left(s^{\prime \prime}, F^{\prime \prime}\right) \in G$ extensão comum de $(s, F)$ e $\left(s^{\prime}, F^{\prime}\right)$. Assim, temos que $s^{\prime} \cap X \subseteq s^{\prime \prime} \cap X \subseteq s$. Como isso vale para todo $s^{\prime}$ dessa forma, segue que $D \cap X \subseteq s$.

Agora fixe $Y \in \mathcal{C}$. Fixe $n$ natural. Existe $(s, F) \in G \cap E_{n}^{Y}$. Temos que $s \cap Y \nsubseteq n$ e $s \cap Y \subseteq D \cap Y$. Assim, $D \cap Y \nsubseteq n$. Como $n$ é arbitrário, segue que $D \cap Y$ é infinito.

\subsection{Topologia da ordem e princípios combinatórios}

Definição 1.9. Uma ordem linear, ou ordem total é um par $(P, \leq) \operatorname{com} P$ não vazio e $\leq$ uma relação em $P$ que satisfaz as seguintes propriedades:

(a) Para todo $a \in P, a \leq a$.

(b) Para todos $a, b, c \in P$, se $a \leq b$ e $b \leq c$ então $a \leq c$.

(c) Para todos $a, b \in P$, se $a \leq b$ e $b \leq a$ então $a=b$

(d) Para todos $a, b \in P$, temos que $a \leq b$ ou $b \leq a$.

Espera-se que o leitor tenha familaridade com as noções de ordem total estrita induzida por uma ordem total.

Definição 1.10. Seja $P$ uma ordem total e $a, b \in P$. Define-se:

(a) $(a, b)=\{x \in P: a<x<b\}$

(e) $(-\infty, b)=\{x \in P: x<b\}$

(b) $(a, b]=\{x \in P: a<x \leq b\}$

(f) $(-\infty, b]=\{x \in P: x \leq b\}$

(c) $[a, b)=\{x \in P: a \leq x<b\}$

(g) $(a, \infty)=\{x \in P: a<x\}$

(d) $[a, b]=\{x \in P: a \leq x \leq b\}$

(h) $[a, \infty)=\{x \in P: a \leq x\}$

Note que os símbolo $\infty$ e $-\infty$ fazem apenas parte da notação, e não são elementos de $P$. A notação acima será utilizada mesmo para conjuntos que tenham menor e maior elemento.

Definição 1.11. Seja $(P, \leq)$ uma ordem total. A topologia da ordem em $P$ é a topologia gerada pelos conjuntos do tipo $(-\infty, b)$ e $(a, \infty)$ para $a, b \in P$.

A demonstração da proposição abaixo fica a cargo do leitor.

Proposição 1.14. Na notação acima, se $|P|>1$ temos que se $a=\min P$, então uma base local para $a$ é $\{[a, b): b>a\}$. Se $a=\max P$, uma base local para $a$ é $\{(b, a]: b<a\}$, e se $a$ não é mínimo nem máximo de $P$, uma base local para $a$ é $\{(b, c): b<a<c\}$. Além disso, intervalos abertos são todos abertos, e intervalos fechados são todos fechados.

Proposição 1.15. Seja $X$ um espaço topológico. Temos que $X$ é hereditariamente normal se, e somente se, para todos $A, B \subseteq X$, se cl $A \cap B=\operatorname{cl} B \cap A=\emptyset$ então existem $U, V \subseteq X$ abertos disjuntos com $A \subseteq U, B \subseteq V$.

Demonstração. Seja $Y \subseteq X$ subespaço. Tome $A, B$ fechados em $Y$ disjuntos. Temos que $\operatorname{cl} A \cap Y$ e $\operatorname{cl} B \cap Y$ são disjutos. Temos que $\operatorname{cl}(\operatorname{cl} A \cap Y) \cap(\operatorname{cl} B \cap Y) \subseteq \operatorname{cl}(\operatorname{cl} A) \cap \operatorname{cl} Y \cap \operatorname{cl} B \cap Y=\operatorname{cl} A \cap$ $Y \cap \operatorname{cl} B \cap Y=\emptyset$. Analogamente, $\operatorname{cl}(\operatorname{cl} B \cap Y) \cap(\operatorname{cl} A \cap Y)=\emptyset$. Assim, existem $U, V \subseteq X$ abertos disjuntos com cl $A \cap Y \subseteq U$, cl $B \cap Y \subseteq V$. Considere os abertos em $Y$ dados por $U \cap Y, V \cap Y$.

Reciprocamente, suponha que $X$ é hereditariamente normal e suponha que $\operatorname{cl} A \cap B=\operatorname{cl} B \cap$ $A=\emptyset$. Temos que cl $A \cap(A \cup B)=A$ e $\operatorname{cl} B \cap(A \cup B)=B$ são disjuntos. Assim, temos que $\operatorname{cl} A \cap(A \cup B)=A$ e $\operatorname{cl} B \cap(A \cup B)=B$ são fechados disjuntos de $A \cup B$. Assim, existem $U, V$ abertos em $X$ tais que $A \subseteq U$ e $B \subseteq V$ e $U \cap V \cap(A \cup B)=\emptyset$. Considere os abertos $U \backslash \mathrm{cl} B$, $V \backslash \operatorname{cl} A$. 
Definição 1.12. Seja $(P, \leq)$ uma ordem total munida da topologia da ordem. Então $P$ é Hausdorff e hereditariamente normal.

Demonstração. Para ver que $P$ é Hausdorff, suponha que $a, b$ são elementos distintos de $P$. Sem perda de generalidade, $a<b$. Se existe $c$ tal que $a<c<b$, considere $(-\infty, c)$ e $(c, \infty)$. Caso contrário, considere $(-\infty, b)$ e $(a, \infty)$.

Para ver que $P$ é hereditariamente normal, suponha que $A \cap \operatorname{cl} B=\operatorname{cl} A \cap B=\emptyset$. Veremos que existem abertos disjuntos $U, V \operatorname{com} A \subseteq U, B \subseteq V$.

Primeiro, vamos supor que $A, B$ não contém extremos de $P$, ou seja, $\min P, \max P \notin A \cup B$ (se existirem).

Para cada $a \in A$, escolha $p_{\alpha}, q_{\alpha}$ satisfazendo:

(a) $p_{a}<a<q_{a}$,

(b) $\left(p_{a}, q_{a}\right) \cap B=\emptyset$

(c) $\left(a, q_{a}\right)=\emptyset$ ou $q_{a} \in A$ ou $\left(q_{a} \notin B\right.$ e $\left.\left(a, q_{a}\right) \cap A=\emptyset\right)$.

(d) $\left(p_{a}, a\right)=\emptyset$ ou $p_{a} \in A$ ou $\left(p_{a} \notin B\right.$ e $\left.\left(p_{a}, a\right) \cap A=\emptyset\right)$.

Para ver que existem $p_{a}, q_{a}$, como $A \cap \operatorname{cl} B=\emptyset$, existem $p, q \operatorname{com} p<a<q$ e $(p, q) \cap B=\emptyset$. Se $(a, q)=\emptyset$, seja $q_{a}=q$. Caso contrário, se $(a, q) \cap A \neq \emptyset$, tome $q_{a} \in(a, q) \cap A$. Caso contrário, tome $q_{a} \in(a, q)$ qualquer. Analogamente, escolhe-se $p_{a}$.

Seja $U=\bigcup_{a \in A}\left(p_{a}, q_{a}\right)$. Temos que $A \subseteq U$ e $B \cap U=\emptyset$. Seja $V=P \backslash$ cl $U$. Temos que $V$ é aberto e $U \cap V=\emptyset$. Resta ver que $B \subseteq V$. Para isso, veremos que $B \cap \operatorname{cl} U=\emptyset$. Para isso, basta ver que dado $b \in B$, existe uma vizinhança de $b$ que não intersecta $U$. Fixe $b$. Como $B \cap \operatorname{cl} A=\emptyset$, tome $u, v \in P \operatorname{com} u<b<v$ e $(u, v) \cap A \neq \emptyset$. Suponhamos que $(u, b)$ e $(b, v)$ sejam não vazios. Assim, podemos supor, encolhendo $(u, v)$ se necessário, que $u, v \notin A$. Suponha por absurdo que $(u, b) \cap U$. Temos que existe $a$ com $(u, b) \cap\left(p_{a}, q_{a}\right)=\emptyset$. Tome $x$ na interseção: Dividiremos a prova em casos:

Caso 1: $u \leq p_{a}$ : como $a \in A$, temos que $a \notin(u, v)$. Porém, $p_{a}<a$, assim, $v \leq a$. Logo, temos que $u \leq p_{a}<v \leq a$. Como $x \in(u, v) \cap\left(p_{a}, q_{a}\right)$, temos que $p_{a}<x<v$. Logo, temos que $u \leq p_{a}<x<v \leq a$. Note que $\left(p_{a}, a\right) \neq \emptyset$. Além disso, temos que $p_{a} \notin A$, ou teríamos que $(u, v) \cap A \neq \emptyset$ ou $u \in A$. Assim, devemos ter que $\left(p_{a}, a\right) \cap A=\emptyset$ (de (d)), o que implica que $x \notin a$. Absurdo.

Caso 2: $p_{a}<u$ : como $a \in A$, temos que $p_{a}<u<v<a$. Mas então $b \in\left(p_{a}, q_{a}\right)$, absurdo.

Analogamente, $(b, v) \cap U \neq \emptyset$ gera um absurdo. Se, inicialmente, tivessemos $(u, b)=\emptyset$, bastaria considerar o que acontece com $(b, v)$, e vice-versa. Se ambos forem vazios, temos que $b$ é aberto e não intersecta $U$.

Resta apenas discutir o que acontece se extremos de $P$ estão em $A \cup B$. Se $x=\min P$ está em $A \cup B$ e o máximo não está/não existe, sem perda de generalidade vamos supor que $x \in A$. Se $x$ é ponto isolado, Tomemos $U, V$ que separam $A \backslash\{x\}, B$, e consideremos $U \cup\{x\}, V \backslash\{x\}$. Se $x$ não for ponto isolado, como $A \cap \operatorname{cl} B=\emptyset$, existe $c \operatorname{com}[x, c) \cap B=\emptyset$. Tomemos abertos $U, V$ separando $(A \backslash\{x\}) \cup(x, c), B$. Temos que $x \notin V$, ou teríamos que $V \cap(x, c) \neq \emptyset$, e como $(x, c) \subseteq U$, temos que $U \cup\{x\}$ continua aberto. Considere $U \cup\{x\}, V$.

Porém, a topologia da ordem pode não ser perfeitamente normal, ou seja, pode não ser ao mesmo tempo perfeito (todo fechado é um $G_{\delta}$ ) e normal, como ocorre no exemplo abaixo.

Proposição 1.16. $\omega_{1}+1$ não é perfeitamente normal.

Demonstração. $\left\{\omega_{1}\right\}$ é um fechado que não é um $G_{\delta}$ : Todo aberto contendo $\left\{\omega_{1}\right\}$ é co-enumerável, portanto, qualquer interseção enumerável destes é não enumerável.

Como cultura geral, provaremos uma condição necessária e suficiente para que uma topologia da ordem seja conexa. 
Definição 1.13. Seja $P$ uma ordem total. Seja $A \subseteq P$. Dizemos que $b \in P$ é limitante superior de $A$, ou majorante de $A$, ou cota superior de $A$, se $\forall a \in A(a \leq b)$. Dizenos que um majorante $b$ de $A$ é supremo de $A$ é um majorante de $A$ tal que $\forall c \in P$, se $c$ é majorante de $A$ então $b \leq c$. É fácil mostrar que caso exista, o supremo de $A$ é único e é denotado sup $A$. Analogamente, define-se cota inferior/limitante inferior/minorante e $\inf A$.

$A \subseteq P$ é limitado superiormente se possui um majorante, e limitado inferiormente se possui um minorante.

Dizemos que $P$ possui a propriedade do supremo se para todo $A \subseteq P$, se $A$ é não vazio e limitado superiormente então $A$ possui um supremo.

Dizemos que $P$ é denso em si mesmo se para todos $a, b \in P$, se $a<b$ então existe $c \in P$ com $a<c<b$.

O lema abaixo fica a cargo do leitor:

Lema 1.17. Seja $P$ uma ordem total e $a, b \in P \operatorname{com} a<b$. Então a topologia de subespaço de $[a, b]$ induzida pela topologia da ordem em $P$ coincide com a topologia da ordem em $P$.

Note que uma generalização do fato acima nem sempre é válida: Considere $[0,1) \cup\{2\} \subseteq \mathbb{R}$.

Agora introduziremos noções importantes para enunciar alguns princípios combinatórios.

Definição 1.14. Seja $\alpha$ um ordinal munido da topologia da ordem. $C \subseteq \alpha$ é um club (closed and unbounded) se, e somente se, for fechado e ilimitado. $S \subseteq \alpha$ é não-estacionário se existe um club $C \subseteq \alpha \operatorname{com} C \cap S=\emptyset$. Caso contrário, $S$ é dito não estacionário.

Define-se ainda $\operatorname{Club}(\alpha)$ como a coleção dos subconjuntos de $\alpha$ que contém um club.

Lema 1.18. Seja $\alpha$ um ordinal. $C \subseteq \alpha$ é fechado se, e somente se, for ilimitado em $\alpha$ e

$$
\forall \beta \in \alpha(C \cap \beta \text { é ilimitado em } \beta \rightarrow \beta \in C)
$$

Demonstração. Suponha que $C$ é fechado e seja $\beta<\alpha$ ordinal limite tal que $C \cap \alpha$ é ilimitado em $\alpha$. Uma base local de $\beta$ são intervalos do tipo $(\gamma, \beta] \operatorname{com} \gamma<\beta$. Dado $\gamma$, por hipótese temos que $C \cap(\gamma, \beta] \neq \emptyset$. Assim, $\beta \in \operatorname{cl} C=C$.

Reciprocamente, suponha que essa propriedade vale para $C$. Veremos que $C$ é fechado. Tome $\beta \in \operatorname{cl} C$. Se $\beta 0$ ou for um ordinal sucessor, temos que $\beta$ é um ponto isolado, portanto, $\beta \in C$. Caso contrário, $\beta$ é limite. Suponha por absurdo que $\beta \notin C$. Veremos que $\beta \cap C$ é ilimitado em $\beta$, e assim, seguirá um absurdo. Dado $\gamma<\beta$, temos que $(\gamma, \beta]$ é aberto. Como $\beta \in \operatorname{cl} C$, temos que $(\gamma, \beta] \cap C \neq \emptyset$. Logo, existe $\delta \in C \operatorname{com} \gamma<\delta \leq \beta$. Como $\beta \notin C$, temos que $\gamma<\delta<\beta$, e, assim, segue a tese.

Proposição 1.19. Suponha que $\mu$ é limite e que $\kappa=\operatorname{cf}(\mu)>\omega$. Então uma interseção de menos de $\kappa$ clubs de $\alpha$ é um club. Consequentemente, $\operatorname{Club}(\mu)$ é um filtro $\operatorname{cf}(\mu)$-completo.

Demonstração. Seja $\lambda<\kappa$ um cardinal infinito. Para cada $\alpha<\lambda$ seja $C_{\alpha}$ um club em $\mu$. Seja $D=\bigcap_{\alpha<\lambda} C_{\alpha}$. Como $D$ é uma interseção de fechados, segue que $D$ é fechado. Resta ver que $D$ é ilimitado. Dado $\xi<\mu$ e seja $f_{\alpha}(\xi) \in C_{\alpha}$ maior que $\xi$. Seja $g(\xi)=\sup \left\{f_{\alpha}(\xi): \alpha<\lambda\right\}$. Como $\lambda<\kappa$, segue que $g(\xi)<\mu$. Definamos $g^{\omega}(\xi)=\sup \left\{g^{n}(\xi): n \in \omega\right\}$. Temos que $g^{\omega}(\xi)<\mu$ pois $\omega<\kappa$. Fixe $\xi$. Como $g^{n}(\xi)$ é estritamente crescente, temos que $g^{\omega}(\xi)$ é um ordinal limite. Fixado $\alpha$, temos que $C_{\alpha}$ é ilimitado em $g^{\omega}(\xi)$ : dado $\beta<g^{\omega}(\xi)$, existe $n \operatorname{com} \beta<g^{n}(\xi)<f_{\alpha}\left(g^{n}(\xi)\right)<g^{n+1}(\xi)<g^{\omega}(\xi)$, e $f_{\alpha}\left(g^{n}(\xi)\right) \in C_{\alpha}$. Assim, como $C_{\alpha}$ é fechado, temos que $g^{\omega}(\xi) \in C_{\alpha}$ e $<g^{\omega}(\xi)>\xi$. Como $\alpha$ é arbitrário, $<g^{\omega}(\xi) \in D$. Como $\xi$ é arbitrário, $D$ é ilimitado.

Note que a condição $\operatorname{cf}(\mu)>\omega$ é realmente necessária, caso contrário, sendo $\alpha_{n}(n \in \omega)$ uma sequência estritamente crescente e cofinal em $\mu$, temos que $\left\{\alpha_{2 n}: n \in \omega\right\}$ e $\left\{\alpha_{2 n+1}: n \in \omega\right\}$ são dois clubs disjuntos.

Corolário 1.20. Suponha que $\mu$ é limite e que $\operatorname{cf}(\mu)>\omega$. Então $S \subseteq \alpha$ é estacionário, se, e somente se, $S \notin \operatorname{Club}(\mu)^{*}$, sendo este último o ideal dual de $\operatorname{Club}(\mu)$. 
Definição 1.15. $\diamond$ é a afirmação: Existe uma família $\left(A_{\alpha}: \alpha<\omega_{1}\right)$ tal que, para cada $\alpha, A_{\alpha} \subseteq \alpha$ e:

$$
\left.\forall A \subseteq \omega_{1}\left(\left\{\alpha<\omega_{1}: A \cap \alpha=A_{\alpha}\right\} \text { é estacionário }\right\}\right) .
$$

Uma sequência dessa forma é chamada de sequência $\diamond$, ou sequência diamante.

Lema 1.21. $\diamond \rightarrow \mathrm{CH}$

Demonstração. Seja $\left(A_{\alpha}: \alpha<\omega_{1}\right)$ uma sequência diamante. Então dado $A \subseteq \omega$, existe $\alpha>\omega$ com $A=A \cap \alpha=A_{\alpha}$. Assim:

$$
\mathscr{P}(\omega)=\left\{A_{\alpha}: A_{\alpha} \subseteq \omega\right\}
$$

o que implica que $|\mathscr{P}(\omega)|<\omega_{1}$.

Antes de terminarmos a seção, enunciaremos e provaremos o Lema de Fodor, também conhecido como Pressing Down Lemma.

Lema 1.22. Suponha que $\kappa>\omega$ é regular e seja $\mathcal{A}$ uma coleção de menos do que $\kappa$ funções finitárias em $\kappa$ (ou seja, cada $f \in \mathcal{A}$ tem domínio $\kappa^{n}$ para algum $n$ natural, e imagem $\kappa$ ). Então o conjunto abaixo é um club:

$$
C=\left\{\gamma<\kappa: \forall f \in \mathcal{A}\left(f: \kappa^{n} \rightarrow \kappa \text { e } f\left[\gamma^{n}\right] \subseteq \gamma\right)\right\}
$$

$(C$ é o conjunto dos ordinais fechados por $\mathcal{A})$.

Demonstração. Para ver que $C$ é fechado, suponha que $\gamma$ é um ordinal limite tal que $C \cap \gamma$ é ilimitado em $\gamma$. Fixe $f \in \mathcal{A}$ função $n$-ária. Se $\beta_{1}, \ldots, \beta_{n}<\gamma$ temos que existe $\beta \in \mathcal{C} \cap \gamma$ com $\beta_{1}, \ldots, \beta_{n}<\beta$. Logo, $f\left(\beta_{1}, \ldots, \beta_{n}\right)<\beta<\gamma$. Isso mostra que $f\left[\gamma^{n}\right] \subseteq \gamma$, e, assim, $\gamma \in C$. Para ver que $C$ é ilimitado em $\kappa$, tome $\xi<\kappa$. Seja $\xi_{0}=\xi$. Definido $\xi_{n}<\kappa$, seja $\sup g\left(\xi_{n}\right)<\xi_{n+1}<\kappa, g\left(\xi_{n}\right)$ é o fecho por $\mathcal{A}$ de $\xi_{n}$, que, pelo Lema 1.9, tem cardinalidade $<\kappa$, e, assim, pela regularidade de $\kappa$, $\sup g\left(\xi_{n}\right)<\kappa$. Por fim, seja $\xi_{\omega}=\bigcup_{n \in \omega} \xi_{n}<\kappa$. Temos que $\xi_{\omega}>\xi$ é fechado por $\mathcal{A}$.

Lema 1.23. Seja $\kappa>\omega$ regular e seja $\left(C_{\alpha}\right)_{\alpha<\kappa}$ uma família de clubs. Então a interseção diagonal da família, definida abaixo, é um club.

$$
D=\left\{\gamma<\kappa: \forall \alpha<\gamma\left(\gamma \in C_{\alpha}\right)\right\}
$$

Demonstração. Veremos primeiro que $D$ é fechado. Se $\gamma$ é limite e $D \cap \gamma$ é ilimitado em $\gamma$, veremos que $\gamma \in D$. Para isso, devemos ver que se $\alpha<\gamma$ então $\gamma \in C_{\alpha}$. Então suponha que $\alpha<\gamma$. Devemos ver que $\gamma \in C_{\alpha}$. Como $C_{\alpha}$ é fechado e $\gamma$ é limite, basta ver que $\gamma \cap C_{\alpha}$ é ilimitado em $C_{\alpha}$. Ora, se $\xi<\gamma$, como $D \cap \gamma$ é ilimitado em $\gamma$, existe $\beta<\gamma \operatorname{com} \beta>\xi, \alpha$ e $\beta \in D$. Logo, $\beta \in C_{\alpha}$. Como $\xi$ é arbitrário, $\gamma \cap C_{\alpha}$ é ilimitado em $\gamma$, então $\gamma \in C_{\alpha}$. Como $\alpha<\gamma$ é arbitrário, segue a tese.

Para ver que $D$ é ilimitado, fixe $\xi$. Seja $\xi_{0}=\xi$. Definido $\xi_{n}<\kappa$, como $\xi_{n}<\mathrm{c}(\kappa)=\kappa$, temos que $\bigcap_{\alpha<\xi_{n}}$ é um club. Tome $\xi_{n+1}>\xi_{n}$. Seja $\xi_{\omega}=\bigcup_{n \in \omega} \xi_{n}<\kappa$. Temos que $\xi_{\omega}>\xi$ e $\xi_{\omega} \in D$. Para ver essa última afirmaçãoo, dado $\alpha<\xi_{\omega}$, temos que existe $n$ tal que $\xi_{n}>\alpha$. Daí, a sequência $\left(\xi_{m}\right)_{m>n}$ mostra que $\xi_{\omega} \cap C_{\alpha}$ é ilimitado em $\xi_{\omega}$, assim, $\xi_{\omega} \in C_{\alpha}$.

Lema 1.24 (Lema de Fodor). Seja $\kappa>\omega$ um cardinal regular, $S \subseteq \kappa$ estacionário e $f: S \rightarrow \kappa$ tal que $\forall \gamma \in S(f(\gamma)<\gamma)$. Então existe $\alpha<\kappa$ tal que $f^{-1}[\{\alpha\}]$ é estacionário.

Demonstração. Suponha que não. Para cada $\alpha$, tome $C_{\alpha}$ club com $C_{\alpha} \cap f^{-1}[\{\alpha\}]=\emptyset$. Seja $D=$ $\left\{\gamma: \forall \alpha<\gamma\left(\gamma \in C_{\alpha}\right)\right\}$. Pelo lema anterior, $D$ é um club. Porém, temos que $D \cap S=\emptyset$, pois se $\gamma \in D \cap S$, temos que $f(\gamma) \neq \alpha(\forall \alpha<\gamma)$, contradizendo que $f(\gamma)<\gamma$. Absurdo. 


\subsection{Espaços conexos, zero Dimensionais e totalmente desconexos}

Espera-se que o leitor tenha alguma familiaridade com a noção de conexidade. Nesta seção, revisaremos algumas outras noções relacionadas a esta.

Definição 1.16. Um espaço topológico $X$ é dito conexo se os únicos clopens de $X$ são $X$ e $\emptyset$.

Proposição 1.25. Seja $X$ um espaço topológico e seja $\mathcal{A}$ uma coleção de subespaços conexos de $X$ tais que para todo $A, B \in X$, temos que $A \cap B \neq \emptyset$. Então $\bigcup \mathcal{A}$ é conexo.

Demonstração. Seja $Y=\bigcup \mathcal{A}$. Seja $C$ um clopen não vazio de $Y$, e seja $D=Y \backslash C$. Veremos que $C=Y$ e $D=\emptyset$.

Dado $A \in \mathcal{A}$, temos que $A \subseteq C$ ou $A \subseteq D$ : Se $A \cap C \neq \emptyset$, temos que $A \cap C$ é clopen não vazio de $A$, logo, $A \cap C=A$, assim, $A \subseteq C$. Analogamente, se $A \cap D \neq \emptyset$ temos que $A \subseteq D$. Como $A \cap(C \cup D)=A$, segue a tese.

Sejam $\mathcal{C}=\{A \in \mathcal{A}: A \subseteq C\}, \mathcal{D}=\{A \in \mathcal{A}: A \subseteq D\}$. Temos que $\mathcal{C}, \mathcal{D}$ particionam $\mathcal{A}$. Como $C \neq \emptyset$, temos que $\mathcal{C} \neq \emptyset$. Tome $A \in \mathcal{C}$. Temos que $\mathcal{D}=\emptyset$, pois se $B \in \mathcal{D}$, temos que $A \cap B \neq \emptyset$. Porém $A \cap B \subseteq C \cap D=\emptyset$. Logo, $\mathcal{C}=\mathcal{A}$, assim, $C \subseteq \bigcup \mathcal{A}=\bigcup \mathcal{C} \subseteq C$, e $D=\emptyset$.

Proposição 1.26. Seja $A \subseteq X$ conexo e suponha que $A \subseteq B \subseteq \operatorname{cl} A$. Então $B$ é conexo.

Demonstração. Suponha que $C \subseteq B$ é um clopen não vazio de $B$. Como $A$ é denso em $B$ e $C$ é aberto em $B$, temos que $A \cap C \neq \emptyset$. Como $C \cap A$ é clopen não vazio de $A$, temos que $C \cap A=A$. $\operatorname{logo}, A \subseteq C$. Como $C$ é fechado em $B$ e contém o denso $A$, temos que $C=B$.

Definição 1.17. Seja $X$ um espaço topológico. Para todo $C_{x}$, a componente conexa de $x$ é o conjunto dado por:

$$
C_{x}=\bigcup\{A \subseteq X: x \in A \text { e } A \text { é conexo }\}
$$

Um espaço topológico $X$ é dito totalmente desconexo se os únicos subespaços conexos de $X$ são os unitários.

Proposição 1.27. Dado $x \in X, C_{x}$ é conexo e fechado. Além disso, $X$ é a união disjunta de suas componentes conexas.

Demonstração. Temos que $C_{x}$ é conexo pois é uma união de conexos com um ponto em comum (o ponto $x) . C_{x}$ é fechado pois $\operatorname{cl} C_{x}$ é conexo, logo, $\operatorname{cl} C_{x} \subseteq C_{x} . X$ é a união de suas componentes conexas pois para todo $x \in X, x \in C_{x}$, e a união é disjunta pois se $C_{x} \neq C_{y}$, temos que $C_{x} \cap C_{y}=\emptyset$, ou teremos que $C_{x} \cup C_{y}$ é conexo, logo, $C_{x} \cup C_{y} \subseteq C_{x}$ e $C_{x} \cup C_{y} \subseteq C_{y}$, assim, $C_{x}=C_{y}$.

Nosso objetivo agora é provar uma equivalência útil para trabalhar-se com espaços totalmente desconexos. Para o restante da seção, usaremos as seguintes relações:

Definição 1.18. Seja $X$ um espaço topológico. Dizemos que $x \sim y$ se, e somente se, existe $C \subseteq X$ conexo com $x, y \in C$. Dizemos que $x \approx y$ se não existe $C \subseteq X$ clopen com $x \in C, y \in X \backslash C$.

É fácil verificar que as relações acima são relações de equivalência, que as classes de equivalência de $\sim$ são as componentes conexas, e que a classe de $x$ por $\approx$ é dada por $D_{x}=\bigcap\{A \subseteq X$ : $A$ é clopen e $x \in A\}$. Os conjuntos $D_{x}$ são as quase-componentes de $X$. A proposição abaixo é de fácil demonstração e fica como exercício:

Proposição 1.28. Se $x \sim y$ então $x \approx y$. Logo, para todo $x, C_{x} \subseteq D_{x}$.

Seguiremos agora para a seguinte proposição:

Proposição 1.29. Suponha que $X$ é compacto Hausdorff. Então $D_{x}$ é conexo (o que implica em $\left.D_{x}=C_{x}\right)$. 
Demonstração. Suponha que $X_{1}, X_{2} \subseteq D_{x}$ são fechados disjuntos tais que $X_{1} \cup X_{2}=D_{x}$. Sem perda de generalidade, $x \in X_{1}$. Veremos que $X_{2}=\emptyset$.

Como $X_{1}, X_{2}$ são fechados disjuntos do espaço normal $X$, temos que existem $U, V \subseteq X$ abertos disjuntos com $X_{1} \subseteq U, X_{2} \subseteq V$. Pela compacidade de $X$, existe uma coleção finita $A_{1}, \ldots, A_{n}$ de clopens com $x$ em cada um deles tal que $D_{x} \subseteq \bigcap_{i=1}^{n} A_{i} \subseteq U \cap V$. Seja $F=\bigcap_{i=1}^{n} A_{i}$. Temos que $F$ é clopen. Veremos que $U \cap F$ é clopen, o que implicará em que $D_{x} \subseteq U \cap F$, o que implica em $X_{2}=\emptyset$.

Temos que $x \in U \cap F$ e que $U \cap F$ é aberto. Para ver que $U \cap F$ é fechado, note que $\operatorname{cl}(U \cap F) \subseteq$ $(\operatorname{cl} U) \cap F=(\operatorname{cl} U) \cap(U \cup V) \cap F=U \cap F$.

Corolário 1.30. Se $X$ é compacto Hausdorff, então $X$ é totalmente desconexo se, e somente se, quaisquer dois pontos distintos são separados por clopens, ou seja, se dados $x, y \in X \operatorname{com} x \neq y$, existe $D$ clopen tal que $x \in D$ e $y \notin D$.

Finalmente, definiremos os espaços zero-dimensionais.

Definição 1.19. Seja $X$ um espaço topológico. Dizemos que $X$ é zero-dimensional se, e somente se, o conjunto de todos os clopens é uma base de $X$.

Proposição 1.31. Seja $X$ um espaço Hausdorff. Então $X$ se zero-dimensional, $X$ é totalmente desconexo. Se, além disso, $X$ é localmente compacto, então vale a recíproca.

Demonstração. Como $X$ é Hausdorff, temos que a condição de zero-dimensionalidade implica que para todo $x, C_{x}=D_{x}=\{x\}$. Assim, $X$ é totalmente desconexo. Agora suponha que $X$ é localmente compacto. Tome $x \in X$ e $V$ vizinhança aberta de $x$. Seja $W$ vizinhança aberta de $x$ tal que $x \in W \subseteq \operatorname{cl} W \subseteq V$ e tal que $\mathrm{cl} W$ é compacto. Temos que, como $\mathrm{cl} W$ é totalmente desconexo, $\{x\}$ é a interseção de todos os clopens de $\mathrm{cl} W$ para os quais $x$ é elemento. Por compacidade, existe um número finito $F_{1}, \ldots, F_{n}$ de clopens de $\operatorname{cl} W$ tais que $x \in F_{1} \cap \cdots \cap F_{n} \subseteq W \subseteq V$.

Sumarizando, temos o seguinte corolário:

Corolário 1.32. Seja $X$ um espaço compacto Hausdorff. São equivalentes:

a) $X$ é totalmente desconexo.

b) $X$ é zero dimensional.

c) Quaisquer dois pontos distintos de $X$ são separados por clopens.

\subsection{Compactificações}

Nessa seção, revisaremos as construções das compactificações de Stone-Čech e da compactificação por um ponto. Seguimos elementos de [Wil04] e [Wal75].

Definição 1.20. Um espaço topológico de Tychonoff é um espaço topológico Hausdorff tal que para todo $x \in X$ e para todo $F \subseteq X$ fechado com $x \notin F$ existe $f: X \rightarrow[0,1]$ contínua tal que $f(x)=0$ e $f[F] \subseteq\{1\}$.

Definição 1.21. Uma compactificação de um espaço topológico $X$ é um par $(K, e)$ onde $K$ é um espaço compacto Hausdorff e $e: X \rightarrow K$ é uma imersão topológica densa.

Note que, como a propriedade de ser um espaço de Tychonoff é hereditária, se um espaço possui compactificação, ele é Tychonoff (pois, pelo lema de Urysohn todo espaço compacto Hausdorff é Tychonoff). Reciprocamente, construiremos a seguir a compactificação $(\beta X, e)$ para $X$ Tychonoff. 
Definição 1.22. Sejam $(K, e),(L, i)$ compactificações de $X$. Dizemos que $(K, e) \leq(L, i)$ se existe uma função contínua $F: L \rightarrow K$ tal que $F \circ i=e$. Dizemos que $(K, e) \approx(L, i)$ (e dizemos que as compactificações são topologicamente equivalentes) se, e somente se, na notação anterior, $F$ é um homeomorfismo.

Proposição 1.33. Seja $X$ um espaço topológico e $(K, e),(L, i)$ compactificações de $X$. Então $(K, e) \approx(L, i)$ se, e somente se, $(K, e) \leq(L, i)$ e $(L, i) \leq(K, e)$.

Além disso, $\leq \mathrm{e} \approx$ são relações reflexivas, transitivas e $\approx$ é simétrica.

Demonstração. Se $(K, e) \approx(L, i)$, é claro que $(K, e) \leq(L, i)$ e $(L, i) \leq(K, e)$. Reciprocamente, suponha que $(K, e) \leq(L, i)$ e $(L, i) \leq(K, e)$. Tome $F, G$ funções contínuas tais que $F \circ e=i$ e $G \circ i=f$. Temos que $G \circ i=G \circ F \circ e=e$, o que mostra que $G \circ F \mid e[X]$ é a identidade. Mas como $e[X]$ é denso em $K$, temos que $G \circ F$ é a identidade. Da mesma forma, $F \circ G$ é a identidade, logo, $F, G$ são homeomorfismos inversos.

As outras afirmações ficam a cargo do leitor.

Verificaremos adiante o fato bem conhecido de que a compactificação de Stone-Čech é uma compactificação maximal nessa ordem.

Lema 1.34. Seja $S$ um espaço Hausdorff, $f: S \rightarrow T$ contínua e $A \subseteq S$ denso tal que $f \mid A$ é um homeomorfismo. Então $f[S \backslash A] \subseteq T \backslash f[A]$.

Demonstração. Suponha por absurdo que não. Tome $z \in f[S \backslash A] \operatorname{com} z \in f[A]$. Temos que $z=f(y)$ para algum $y \in S \backslash A$, e $z=f(x)$ para algum $x \in A$. Temos que $f(x)=f(y)$. Tomemos vizinhanças disjuntas $U, V$ de $x, y$. Como $f \mid A$ é um homeomorfismo, $f[U \cap A]$ é uma vizinhança de $f(x)$ em $f[A]$, então $f[U \cap A]=W \cap f[A]$ onde $W$ é uma vizinhança de $f(x)$ em $T$.

Se $O$ é uma vizinhança de $y$, afirmo que $f[O] \nsubseteq \Phi$. Para ver isso, tome $w \in O \cap V \cap A$. Temos que $f(a) \notin f[U \cap A]$ pois $f \mid A$ é injetora e $V \cap U=\emptyset$. Mas $f(a) \in f[A]$. Assim, $f(a) \notin W$. Logo, $f$ não é contínua em $y$.

Proposição 1.35. Suponha que $(K, e)$ e $(L, i)$ são compactificações de $X$ e que $F \circ i=e$. Então:

(a) $F \mid i[X]$ é um homeomorfismo de $i[X]$ em $e[X]$.

(b) $F[L \backslash i[X]]=K \backslash e[X]$.

Demonstração. Temos que:

(a) $F \mid i[X]=e \circ i^{-1}$, que é um homeomorfismo.

(b) Pelo item acima, $F$ é sobrejetora. Como $F[i[X]] \cap K \backslash e[X]=\emptyset$, temos que $K \backslash e[X] \subseteq$ $F[L \backslash i[X]]$. A desigualdade contrária segue do lema anterior.

\subsubsection{Zero sets}

Definição 1.23. Seja $X$ um espaço topológico. $A \subseteq X$ é um zero set se existe $f: X \rightarrow \mathbb{R}$ contínua tal que $f^{-1}[\{0\}]=A$, é um cozero set se existe $f: X \rightarrow \mathbb{R}$ contínua tal que $f^{-1}[\mathbb{R} \backslash\{0\}]=A$.

O conjunto de todos os zero sets de $X$ é denotado $Z(X)$

Note que $\mathbb{R}$ é tomado com a topologia usual.

Note que o complementar de um zero set é um cozero set e vice versa.

Definição 1.24. Dizemos que $A, B \subseteq X$ são completamente separados se eles estão contidos em zero sets disjuntos.

Se $X \subseteq Y$, dizemos que $X$ é $C$-imerso $\left(C^{*}\right.$-imerso) em $Y$ se para toda $f: X \rightarrow \mathbb{R}$ contínua (e limitada) existe $g: Y \rightarrow \mathbb{R}$ contínua (e limitada) tal que $f \subseteq g$. Além disso, define-se $C(X)$ e $C^{*}(X)$, respectivamente, o conjunto das funções contínuas e das funções contínuas limitadas de $X$ em $\mathbb{R}$. 
Proposição 1.36. Seja $X$ um espaço topológico.

(a) Se $A, B \in Z(X)$, então $A \cup B \in Z(X)$.

(b) Se $A_{n}$ for uma sequência de elementos de $Z(X)$, então $\bigcap_{n \in \omega} A_{n} \in Z(X)$.

Demonstração. Para o primeiro item, considere que $f, g$ atestam respectivamente que $A, B \in Z(X)$ e considere $f . g$. Para o segundo item, considere que $f_{n}$ atesta que $A_{n} \in Z(x)$ e considere

$$
f(x)=\sum_{n \in \omega} \max \left\{\left|f_{n}(x)\right|, \frac{1}{2^{n}}\right\} .
$$

Tomando complementos, obtemos o seguinte corolário:

Corolário 1.37. Seja $X$ um espaço topológico.

(a) Se $A, B$ são cozero sets de $X$, então $A \cap B$ também é.

(b) Se $A_{n}$ for uma sequência de cozero sets de $X$, então $\bigcup_{n \in \omega} A_{n}$ é cozero set de $X$.

Proposição 1.38. Seja $X$ um espaço de Tychonoff. O conjunto dos cozero-sets de $X$ é uma base de $X$.

Demonstração. Sabemos que $X$ é cozero-set e que todos os cozero-sets são abertos. Se $A \subseteq X$ é aberto e $x \in A$, existe $f: X \rightarrow \mathbb{R}$ contínua com $f(x)=1$ e $f[X \backslash A] \subseteq\{0\}$. Temos que $x \in f^{-1}[\mathbb{R} \backslash\{0\}] \subseteq A$.

Proposição 1.39. Sejam $Z, W$ zero sets disjuntos de $X$ e $a, b \in \mathbb{R}$ com $a<b$. Seja $U$ um cozero set de $X$.

(a) Existe $f: X \rightarrow[a, b]$ contínua tal que $Z=f^{-1}[\{a\}]$

(b) Existe $f: X \rightarrow[a, b]$ contínua tal que $Z=f^{-1}[\{b\}]$

(c) Existe $f: X \rightarrow[a, b]$ contínua tal que $Z=f^{-1}[(a, b]]$

(d) Existe $f: X \rightarrow[a, b]$ contínua tal que $Z=f^{-1}[[a, b)]$

(e) Existe $f: X \rightarrow[a, b]$ contínua tal que $Z=f^{-1}[\{a\}], W=f^{-1}[\{b\}]$

(f) Se $A \subseteq X$ é tal que $A=f^{-1}[(a, \infty)]$ para $f$ contínua, então $f$ é cozero set.

(g) Se $A \subseteq X$ é tal que $A=f^{-1}[(-\infty, b)]$ para $f$ contínua, então $f$ é cozero set.

(h) Se $A \subseteq X$ é tal que $A=f^{-1}[(a, b)]$ para $f$ contínua, então $f$ é cozero set.

(i) Se $A \subseteq X$ é tal que $A=f^{-1}[(-\infty, b]]$ para $f$ contínua, então $f$ é zero set.

(j) Se $A \subseteq X$ é tal que $A=f^{-1}[[a, \infty)]$ para $f$ contínua, então $f$ é zero set.

(k) Se $A \subseteq X$ é tal que $A=f^{-1}[[a, b]]$ para $f$ contínua, então $f$ é zero set (inclusive se $a=b$ ).

(1) Se $A, B$ são zero sets disjuntos, existem $C, D$ cozero sets e $E, F$ zero sets com $E \cap F=\emptyset$, $A \subseteq C \subseteq E$ e $B \subseteq D \subseteq F$.

Demonstração. Com a notação do enunciado: 
(a) Tome $g: X \rightarrow \mathbb{R}$ contínua tal que $g^{-1}[\{0\}]=Z$. Considere $f=\min \left\{g^{2}+a, b\right\}$.

(b) Tome $g: X \rightarrow \mathbb{R}$ contínua tal que $g^{-1}[\{0\}]=Z$. Considere $f=\max \left\{-g^{2}+b, a\right\}$.

(c) Análogo.

(d) Análogo.

(e) Seja, $g, h: X \rightarrow \mathbb{R}$ contínuas tais que $g^{-1}[\{0\}]=Z, h^{-1}[\{0\}]=W$. Considere:

$$
f(x)=(b-a) \frac{g^{2}(x)}{g^{2}(x)+h^{2}(x)}+a
$$

(f) Seja $g=\max \{f-a, 0\}$. Temos que $g^{-1}[\mathbb{R} \backslash 0]=f^{-1}[(a, \infty)]$.

(g) Seja $g=\min \{f-b, 0\}$. Temos que $g^{-1}[\mathbb{R} \backslash 0]=f^{-1}[(-\infty, b)]$.

(h) $f^{-1}[(a, b)]=f^{-1}[(a, \infty)] \cap f^{-1}[(-\infty, b)]$.

(i) Seu complemento é cozero set.

(j) Seu complemento é cozero set.

(k) $f^{-1}[[a, b]]=f^{-1}[[a, \infty)] \cap f^{-1}[(-\infty, b]]$.

(1) Pelo item (e), existe $f: X \rightarrow[0,1]$ contínua tal que $f^{-1}[\{0\}]=A$ e $f^{-1}[\{1\}]=B$. Considere $C=f^{-1}[(-1 / 3,1 / 3)], D=f^{-1}[(2 / 3,5 / 3)], E=f^{-1}[[-1 / 3,1 / 3]], F=f^{-1}[[2 / 3,5 / 3]]$.

\subsubsection{Compactificação por um ponto}

Primeiro, apresentaremos a construção de uma compactificação por um ponto. Depois, daremos uma definição mais geral e provaremos a sua unicidade.

Teorema 1.40. Seja $X$ um espaço topológico Hausdorff, localmente compacto, e não compacto. Seja $p$ tal que $p \notin X$, e $\tau$ a topologia de $X$. Seja $Y=X \cup\{p\}$ e seja:

$$
\mathcal{B}=\tau \cup\{\{p\} \cup(X \backslash K): K \subseteq X \text { é compacto }\}
$$

Então $\mathcal{B}$ é uma base para uma topologia em $Y$ que torna $K$ um espaço compacto Hausdorff e $X \subseteq K$ é, com sua topologia original, um subespaço aberto de $Y$.

Demonstração. Vejamos que $\mathcal{B}$ é base para uma topologia em $Y$. Temos que $\bigcup \mathcal{B}=Y$ pois $X \in$ $\tau \subseteq \mathcal{B}$ e fixado $x \in X,(X \backslash\{x\}) \cup\{p\} \in \mathcal{B}$, logo, $p \in \bigcup B$. Se $A, B \in \tau$, temos que $A \cap B \in \tau$. Se $A \in \tau, K \subseteq X$ é compacto, então $\in A \cap((X \backslash K) \cup\{p\})=A \cap(X \backslash K) \in \tau$. Finalmente, Se $K, L$ são compactos, então $K \cup L$ é compacto e $(\{p\} \cup(X \backslash K)) \cap(\{p\} \cup(X \backslash L))=(\{p\} \cup(X \backslash K \cup L)) \in \mathcal{B}$.

Essa topologia é Hausdorff: Se $a, b \in X$ são distintos, esses são separados por elementos de $\tau$, e se $a \in X$, temos que pela compacidade local de $X$, existe $K$ compacto com $x \in \operatorname{int} K \subseteq K$. Assim, $\operatorname{int} K$ e $\{p\} \cup(X \backslash K)$ são abertos disjuntos que separam $a, p$.

Como $\tau \subseteq \mathcal{B}$, temos que $X$ é aberto. Seja $\tau^{\prime}$ a topologia de subespaço de $X$. Temos que $\tau \subseteq \tau^{\prime}$ pois $\tau \subseteq \mathcal{B}$, e temos que todo elemento de $\mathcal{B}$ intersectado com $X$ é um elemento de $\tau$, o que nos dá a inclusão contrária.

Por fim, temos que $Y$ é compacto, pois dada uma cobertura aberta $\mathcal{A}$ de $Y$ de abertos básicos, temos que existe $A \in \mathcal{A}$ com $p \in A$. Como $p \in A$, existe $K \subseteq X$ compacto com $A=(X \backslash K) \cup\{p\}$. Pela compacidade de $K$, existe $\mathcal{C} \subseteq \mathcal{B}$ finito com $K \subseteq \cup \mathcal{C}$. Considere $\mathcal{C} \cup\{A\}$.

Corolário 1.41. Se $X$ é um espaço topológico Hausdorff e localmente compacto, então $X$ é Tychonoff. 
Definição 1.25. Seja $X$ um espaço topológico Tychonoff e $n$ natural. Dizemos que uma compactificação $(K, e)$ de $X$ é uma compactificação por $n$ pontos se $|K \backslash e[X]|=n$.

Notemos que o espaço $Y$ construído no teorema acima é uma compactificação de $X$.

Proposição 1.42. Seja $X$ um espaço topológico Tychonoff. Se $(K, e)$ é uma compactificação por um ponto de $X$, então $X$ é localmente compacto. Além disso, se $(L, f)$ for uma compactificação por um ponto de $X$ então $(K, e) \approx(L, f)$.

Demonstração. Para a primeira afirmação, seja $p \in K \backslash e[X]$. Temos que $\{p\}$ é fechado em $K$, logo, $e[X]$ é aberto. Dado $x \in X$, temos que existe $L \subseteq K$ vizinhança compacta de $e[X]$ com $e(x) \in L \subseteq e[X]$, pois $e[X]$ é aberto e $K$ é compacto. Logo, $x \in e^{-1}[L]$, e esse último é uma vizinhança compacta de $x$.

Para a segunda afirmação, seja $q \in L \backslash f[X]$, seja $\phi$ dada por $\phi(e(x))=f(x)$ para todo $x \in X$ e seja $\phi(p)=q$. Temos que $\phi: K \rightarrow L$ é bijetora, e $\phi \mid e[X]=f \circ e^{-1}$ é contínua. Temos também que $\phi \circ e=f$. Agora vejamos que $\phi$ é contínua em $p$. Seja $A$ uma vizinhança aberta de $q$ em $L$. Temos que $F=L \backslash A \subseteq X$ é compacto. Assim, $G=e \circ f^{-1}[F]$ é compacto. Temos que $K \backslash G$ é vizinhança aberta de $p$ e $\phi[K \backslash G]=L \backslash F=A$, assim, $\phi$ é contínua em $p$. Analogamente, $\phi^{-1}$ é contínua em $q$.

\subsubsection{Compactificação de Stone-Čech}

Nessa seção, seguimos principalmente a construção do livro [CN74]

Definição 1.26. Seja $X$ um espaço topológico. Um z-filtro de $X$ é uma coleção $\mathcal{F}$ não vazia de zero-sets de $X$ fechada para interseção finita e tal que se $A \in \mathcal{F}$ e $B$ é um zero-set com $A \subseteq B$ então $B \in p$. Um z-filtro $p$ é dito próprio se $\emptyset \notin p$, e é um z-ultrafiltro se for um ultrafiltro próprio maximal. O conjunto dos z-ultrafiltros de $X$ é denotado $\beta X$.

Proposição 1.43. Todo z-filtro próprio está contido em um z-ultrafiltro.

Demonstração. Aplicação imediata do lema de Zorn.

Note que se $X$ é discreto, todo subconjunto de $X$ é um zero set e a noção de ultrafiltro coincide com a de z-ultrafiltro, assim como a de filtro (próprio) coincide com a de z-filtro (próprio).

Proposição 1.44. Sejam $p, q$ z-ultrafiltros em $X$.

(a) Se $A \in Z(X)$ e para todo $B \in p, B \cap A \neq \emptyset$ então $A \in p$,

(b) Se $A, B \in Z(X)$ e $A \cup B \in p$ então $A \in p$ ou $B \in p$,

(c) Se $p \neq q$ existem $A \in q, B \in p \operatorname{com} A \cap B=\emptyset$.

Demonstração. Para o item (a), basta considerar $q=\{F \in Z(X): \exists B \in p(A \cap B \subseteq F)\}$. Como $p \subseteq q$ e $q$ é filtro próprio (verifique), segue que $p=q$.

Para o item (b), suponha que $A, B \notin p$. Temos, pelo item (a), que existem $C, D \in p$ com $A \cap C=B \cap D=\emptyset$. Logo, $(A \cup B) \cap C \cap D=\emptyset$, assim, $A \cup B \notin p$, absurdo.

Para o item (c), tome $B \in p \Delta q$. Sem perda de generalidade, $B \in p$. Como $B \notin q$, existe $A \in q$ $\operatorname{com} A \cap B=\emptyset$.

Proposição 1.45. Seja $X$ um espaço de Tychonoff. Para cada $A \subseteq X$ zero set, seja $\tilde{A}=\{p \in \beta X$ : $A \notin p\}$. A coleção $\mathcal{B}=\{\tilde{A}: A$ é zero set de $X\}$ é uma base para uma topologia em $\beta X$.

Demonstração. Temos que $\tilde{\emptyset}=X$ e dados $A, B$ zero sets e $p \in \tilde{A} \cap \tilde{B}$, segue que $A \notin p$ e $B \notin p$. Logo, $A \cup B \notin p$. e $p \in(A \cup B)^{\sim} \subseteq \tilde{A} \cap \tilde{B}$. 
Definição 1.27. Dado $X$ espaço topológico Tychonoff, e seja a função $e: X \rightarrow \beta X$ dada por $e(x)=\{A \in Z(X): x \in A\}$.

Proposição 1.46. Na notação acima, $e: X \rightarrow \beta X$ está bem definida e é uma imersão topológica densa em $\beta X$.

Demonstração. Fixe $x \in X$. Veremos que $e(x)$ é um z-ultrafiltro. Notemos que se $A \in e(x)$ e $B \supset A$ é zero set, temos que $x \in A \subseteq B$, logo, $x \in B$, e, assim, $B \in e(x)$. Suponha que $A, B \in \beta X$. Temos que $x \in A \cap B \in Z(X)$, logo, $A \cap B \in e(x)$. Temos que $e(x) \neq \emptyset$ pois $x \in e(x)$. Assim, $e(x)$ é um z-filtro. Ele é próprio, pois $x \notin \emptyset$, e é um ultrafiltro: Suponha por absurdo que existe $p$ z-filtro que contém propriamente $e(x)$. Então existe zero set não vazio $Z \in p \operatorname{com} x \notin Z$. Então existe $f: X \rightarrow \mathbb{R} \operatorname{com} f(x)=a \neq 0$ e $f[Z]=\{1\}$. Sendo $W=f^{-1}[\{a\}]$, temos que $W \in e(x), \operatorname{logo}$, $W \in p$, mas então $W \cap Z=\emptyset \in p$, absurdo.

Agora vejamos que $e$ é injetora: Se $x \neq y$, tome $f: X \rightarrow \mathbb{R}$ tal que $f(x)=0, f(y)=1$. Temos que $Z=f^{-1}[\{0\}], W=f^{-1}[\{1\}]$ são zero sets disjuntos tais que $Z \in e(x)$ e $W \in e(y)$.

$e$ é contínua: fixe $x \in X$ e tome um aberto básico da forma $\tilde{A} \operatorname{com} e(x) \in \tilde{A}$, para $A \subseteq X$ zero set. Temos que $A \notin e(x), \operatorname{logo}, x \notin A$. Tome $B$ zero set com $x \in B \subseteq X \backslash A$. Se $y \in B$, temos que $e(y) \in \tilde{A}$. Para ver isso, basta ver que $A \notin e(y)$, o que ocorre se, e somente se, $y \notin A$. Ora, temos que $y \in B$, e $B \cap A=\emptyset$.

Seja $A$ um cozero set de $X$. Veremos que $e[A]$ é aberto em $e[X]$. Afirmo que $e[A]=e[X] \cap(X \backslash A)^{\sim}$. Dado $x \in A$, temos que $e(x) \in e[A]$ e $X \backslash A \notin e(x)$, pois $x \in A$. Assim, vale a inclusão $\subseteq$. Para a outra inclusão, se $x$ é tal que $X \backslash A \notin e(x)$, segue que $x \notin X \backslash A, \operatorname{logo}, x \in A$.

Finalmente, temos que $e[X]$ é denso em $\beta X$ : dado um aberto básico $\tilde{A}$ não vazio com $A \subseteq X$ zero set, temos que $A \neq X$, logo, tome $x \in X \backslash A$. Temos que $A \notin e(x)$, logo, $e(x) \in \tilde{A}$.

Corolário 1.47. Se $A$ é zero set de $X, e[A]=e[X] \cap(X \backslash A)^{\sim}$.

Demonstração. Provado na demonstração da proposição anterior.

Proposição 1.48. $\beta X$ é compacto Hausdorff.

Demonstração. Para ver que $\beta X$ é Hausdorff, se $p \neq q$ são z-ultrafiltros temos que existe $A \in p$, $B \in q$ com $A \cap B=\emptyset$. Tomemos cozero sets $C, D$ disjuntos com $A \subseteq C, B \subseteq D$, e zero sets $E, F$ disjuntos com $C \subseteq E$ e $D \subseteq F$. Temos que $p \in(X \backslash C)^{\sim}, q \in(X \backslash D)^{\sim}$. Se $t$ é um z-ultrafiltro tal que $t \in(X \backslash C)^{\sim}$, temos que $E \in t$ pois $X=(X \backslash C) \cup E$. Analogamente, se $t \in(X \backslash D)^{\sim}$, temos que $F \in t$. Como $E \cap F=\emptyset$, as duas vizinhanças apresentadas são disjuntas.

Agora, suponhamos por absurdo que o espaço não é compacto. Então existe $\mathcal{C} \subseteq Z(X)$ tal que $\{\tilde{A}: A \in \mathcal{C}\}$ é uma cobertura aberta sem subcobertura finita. Isso implica que $\{\{p \in \beta X: A \in$ $p\}: A \in \mathcal{C}\}$ é uma coleção de conjuntos com a propriedade da interseção finita cuja interseção é vazia. Isso implica que $\mathcal{C}$ tem a propriedade da interseção finita. Assim, obtém-se um ultrafiltro $q$ que contém $\mathcal{C}$, mas então $q \in \bigcap\{\{p \in \beta X: A \in p\}: A \in \mathcal{C}\}$.

Proposição 1.49. Sejam $A, B \in Z(X)$

(a) $\operatorname{cl} e[A]=\{p \in \beta X: A \in p\}$,

(b) Se $p \in \beta X, p=\{A \in Z(X): p \in \operatorname{cl} e[A]\}$,

(c) $\operatorname{cl} e[A] \cap \operatorname{cl} e[B]=\operatorname{cl} e[A \cap B]$.

(d) $\{\operatorname{cl} e[A]: A \in Z(X)\}$ é uma base de fechados.

Demonstração. Sejam $A, B \in Z(X)$

(a) Por definição, $\{p \in \beta X: A \notin p\}$ é aberto, logo, $\{p \in \beta X: A \in p\}$ é fechado e contém $e[A]$, pois se $x \in A$, temos que $A \in e(x)$. Logo, vale $\subseteq$. Para o contrário, suponha que $A \in p$ mas $p \notin \operatorname{cl} e[A]$. Existe $Z$ zero set com $p \in \tilde{Z}$ mas $\tilde{Z} \cap e[A]=\emptyset$. Assim, $e[A]=e[X] \cap\{t \in \beta X$ : $Z \in t\}=e[Z], \operatorname{logo}, A \subseteq B$, e, assim, $B \in p$, absurdo. 
(b) Segue do item anterior.

(c) Do primeiro item, segue que cle $e[A] \cap \operatorname{cl} e[B]=\{p \in \beta X: A \cap B \in p\}=\operatorname{cl} e[A \cap B]$.

(d) Pelo item (a), temos que a coleção em questão é a coleção dos complementares de nossos abertos básicos.

Lema 1.50. Seja $X \subseteq Y$ denso, $Y$ compacto Hausdorff. Seja $f: Y \rightarrow K$ contínua, com $K$ compacto Hausdorff.

(a) Se para todo $p \in Y$ existe $f_{p}: X \cup\{p\}: \rightarrow \mathbb{K}$ contínua tal que $f \subseteq f_{p}$, então existe $g: Y \rightarrow K$ contínua com $f \subseteq g$.

(b) Se $p \in Y$ é tal que não existe $g: X \cup\{p\} \rightarrow K$ contínua tal que $f \subseteq g$, então existem $A, B \in Z(K)$ disjuntos com $p \in \operatorname{cl} f^{-1}[A] \cap \operatorname{cl} f^{-1}[B]$.

Demonstraçẫo. Para o primeiro item, seja $g(p)=f_{p}(p)$ para todo $p \in Y$. Fixe $p$ e sejam $V, W$ vizinhanças de $g(p)$ tais que $\mathrm{cl} V \subseteq W$. Seja $U$ vizinhança aberta de $p$ tal que $f(q) \in V$ para todo $q \in U \cap X$. Se $q \in U$, então $q \in \operatorname{cl}(U \cap X)=\operatorname{cl} U$. Logo:

$$
g(q)=f_{q}(q) \in \operatorname{cl} f_{q}[U \cap X]=\operatorname{cl} f[U \cap X] \subseteq \operatorname{cl} V \subseteq W .
$$

Para o segundo item, considere:

$$
\mathcal{F}=\{\operatorname{cl} f[U \cap X]: U \subseteq Y \text { é vizinhança aberta de } p\}
$$

$\mathcal{F}$ tem a propriedade da interseção finita, então $\bigcap \mathcal{F} \neq \emptyset$. Se $\bigcap \mathcal{F}=\{s\}$, então $g=f \cup\{(p, s)\}$ é contínua: Suponha por absurdo que não. Tome $W$ vizinhança aberta de $s$ tal que para toda vizinhança aberta $U \subseteq X \cup\{p\}$ de $p$, temos que $f[U] \backslash W \neq \emptyset$. Considere:

$$
\mathcal{G}=\{\operatorname{cl} f[U \cap X] \backslash W: U \subseteq Y \text { é vizinhança aberta de } p\}
$$

$\mathcal{G}$ tem pif, então existe $s^{\prime} \in \bigcap \mathcal{G} \subseteq \bigcap \mathcal{F}$.

Logo, existem $a, b \in \bigcap \mathcal{F}$ distintos. Tome zero sets disjuntos de $K$, denotados por $A, B$ com vizinhanças de $a, b$. Temos que $p \in \operatorname{cl} f^{-1}[A] \cap \operatorname{cl} f^{-1}[B]$, pois se, por exemplo, $p \notin \operatorname{cl} f^{-1}[A]$, temos que existe $U$ vizinhança aberta de $p$ com $U \cap f^{-1}[A]=\emptyset$. Daí, $f[X \cap U] \cap A=\emptyset$, assim, cl $f[X \cap U] \cap \operatorname{int} A=\emptyset$. Logo, $a \notin \operatorname{cl} f[X \cap U]$, absurdo.

Proposição 1.51. Seja $X$ Tychonoff e $(K, e)$ uma compactificação de $X$. São equivalentes:

(a) Para todo espaço compacto Hausdorff $L$ e para toda função $f: X \rightarrow L$ contínua, existe $g: Y \rightarrow L$ contínua tal que $g \circ e=f$.

(b) $e[X]$ é $C^{*}$-imerso em $K$,

(c) Se $Z, W$ são zero sets disjuntos de $X$, então $\operatorname{cl} e[Z] \cap \operatorname{cl} e[W]=\emptyset$,

(d) Se $Z, W$ são zero sets de $X$, então $\operatorname{cl} e[Z] \cap \operatorname{cl} e[W]=\operatorname{cl} e[Z \cap W]$.

(e) Se $p \in K$, então $A_{p}=\{Z \in Z(X): p \in \operatorname{cl} e[Z]\}$ é um z-ultrafiltro em $X$.

Além disso, se $(K, e),(L, f)$ são duas compactificações de $X$ que satisfazem a lista de propriedades acima, então elas são equivalentes. 
Demonstração. A implicação de (a) em (b) é imediata.

Para a implicação de (b) em (c), considere $Z, W$ zero sets disjuntos de $X$. Temos que $e[Z], e[W]$ são zero sets disjuntos de $e[X]$. Logo, existe $f: e[X] \rightarrow[0,1]$ contínua com $e[Z]=f^{-1}[\{0\}]$,

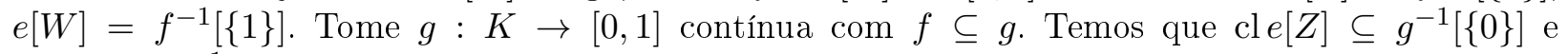
$\operatorname{cl} e[W] \subseteq g^{-1}[\{1\}]$.

Para a implicação de (c) em (d), tome $Z, W$ zero sets de $X$. Está claro que valecl $e[Z] \cap \operatorname{cl} e[W] \supset$ $\operatorname{cl} e[Z \cap W]$. Agora suponha que $p \in K \backslash \operatorname{cle} e[Z \cap W]$. Existe $f: K \rightarrow[0,1] \operatorname{com} f(p)=0$ e $f[e[A \cap B]] \subseteq\{1\}$. Seja $C=(f \circ e)^{-1}\left[\left(-\infty, \frac{1}{2}\right]\right]$. Temos que $A \cap B \cap C=\emptyset$ e $A \cap C, B \cap C \in Z(X)$. Por $(c)$, segue que:

$$
\operatorname{cl} e[A \cap C] \cap \operatorname{cl} e[B \cap C]=\emptyset .
$$

Devemos ter que $p \notin \operatorname{cl} e[A] \cap \operatorname{cl} e[B]$, pois se $p \in \operatorname{cl} e[A]$, temos que $p \in \operatorname{cl}\left(A \cap f^{-1}\left[\left(-\infty, \frac{1}{2}\right)\right]\right)=$ $\operatorname{cl}\left(A \cap f^{-1}\left[\left(-\infty, \frac{1}{2}\right)\right] \cap e[X]\right) \subseteq \operatorname{cl}(A \cap C)$. Analogamente, se $p \in \operatorname{cl}[B]$ temos que $p \in \operatorname{cl}(B \cap C)$.

Para (e), o fato de que $A_{p}$ é z-filtro decorre imediatamente de (d). Provaremos que $A_{p}$ é zultrafiltro. Para isso, veremos que se $W$ é um zero set não vazio de $X$ tal que $x \notin \operatorname{cl} e[W]$, então existe $Z \in A_{p} \operatorname{com} Z \cap W=\emptyset$. Fixe um tal $W$. como $p \notin \operatorname{cl} e[W]$ e cl $e[W]$ é fechado, existe $g: K \rightarrow[0,1]$ contínua com $g(p)=0, g[\operatorname{cl} e[W]]=\{1\}$. Seja $f=g \circ$ e. Seja $Z=f^{-1}\left[\left[0, \frac{1}{3}\right]\right]$. Temos que $p \in \operatorname{cl} e[Z]$, pois $\operatorname{cl} e[Z] \supset \operatorname{cl}\left(g^{-1}\left[\left[0, \frac{1}{3}\right)\right] \cap e[X]\right)=\operatorname{cl}\left(g^{-1}\left[\left[0, \frac{1}{3}\right)\right]\right.$, e $Z \cap W=\emptyset$ pois temos que $\operatorname{cl} e[Z \cap W]=\operatorname{cl} e[Z] \cap \operatorname{cl} e[W]$ e, pelo argumento anterior cl $e[Z] \subseteq g^{-1}\left[0, \frac{1}{3}\right]$, cle $e[W] \subseteq g^{-1}[\{1\}]$.

Para a última implicação, suponha que não vale (a). Então existe um espaço compacto Hausdorff $L$ e $f: X \rightarrow L$ contínua tal que $f \circ e^{-1}: e[X] \rightarrow K$ não pode ser estendida a uma função $g: K \rightarrow L$ contínua. Então existe $p \in K$ tal que não existe $g: e[X] \cup\{p\} \rightarrow L$ contínua com $f \circ e^{-1} \subseteq g$. Então existem $C, D \in Z(L)$ disjuntos com $p \in \operatorname{cl}\left(f \circ e^{-1}\right)^{-1}[C] \cap \operatorname{cl}\left(f \circ e^{-1}\right)^{-1}[D]=$ $\operatorname{cl} e\left[f^{-1}[C]\right] \cap \operatorname{cl} e\left[f^{-1}[D]\right]=\emptyset$, o que viola (e).

Finalmente, se $(K, e)$ e $(L, f)$ são duas compactificações que satisfazem (a)-(e), utilizando (a) para a compactificação $(K, e)$ e para a função $f: X \rightarrow L$, seja $\bar{f}: K \rightarrow L$ contínua tal que $f=\bar{f} \circ e$. De forma análoga, seja $\bar{e}: L \rightarrow K$ contínua com $\bar{e} \circ f=e$. Isso mostra que $(K, e) \leq(L, F)$ e $(L, f) \leq(K, e), \operatorname{logo},(K, e) \approx(L, f)$.

Corolário 1.52. Dado um espaço de Tychonoff $X, \beta X$ é a única compactificação de $X$ a menos de equivalência topológica que satisfaz as propriedades da proposição acima. Além disso, $\beta X$ é a maior compactificação de $X$ a menos de equivalência topológica.

É comum identificar $X$ com $e[X]$ dentro de $\beta X$, considerando que $\beta X$ é um conjunto que possui $X$ como subconjunto e tal que $e$ é a inclusão, ou itentificando $X$ com o conjunto dos z-ultrafiltros fixos de $X$, e considerando que $\beta X$ é o conjunto de todos os z-ultrafiltros de $X$. Nesse documento, fixaremos que $\beta X$ é o conjunto dos ultrafiltros, $e$ a identificação contruída e utilizaremos a notação $X^{*}=\beta X \backslash e[X]$.

Proposição 1.53. Seja $X$ um espaço de Tychonoff. Então $X$ é localmente compacto se, e somente se, $e[X]$ é aberto em $\beta X$.

Demonstração. Se $e[X]$ é aberto em $\beta X$, então $e[X]$, e, portanto, $X$, é localmente compacto, porque todo subespaço aberto de um espaço compacto é localmente compacto. Reciprocamente, se $X$ for localmente compacto, então. Então $X$ admite uma compactificação por um ponto, $X \cup\{p\}$. Seja $f: X \rightarrow X \cup\{p\}$ a inclusão, que é contínua. Tome $F: \beta X \rightarrow X \cup\{p\}$ a extensão contínua de $f$, ou seja, uma função contínua $F$ tal que $F \circ e=f$. Por 1.35 , temos que $\beta X \backslash e[X]=F^{-1}[\{p\}]$, logo, $\beta X \backslash e[X]$ é fechado em $\beta X$, e $e[X]$ é aberto.

Antes de proceder, vamos definir o que são z-filtros livres e fixos.

Definição 1.28. Um z-filtro $p$ em $X$ Tychonoff é dito livre se $\bigcap p=\emptyset$, e é fixo caso o contrário. Além disso, $p$ é dito real se possui a propriedade da interseção enumerável.

Proposição 1.54. Um z-ultrafiltro $p$ é fixo se, e somente se, existe $x \in X$ tal que $p$ é o z-ultrafiltro gerado por $\{x\}$ (ou seja, se $p \in e[X]$ ). 
Demonstração. Se $p$ for o z-ultrafiltro gerado por algum $x \in X$, temos que $\bigcap p=\{x\} \neq \emptyset$. Logo, $p$ é fixo. Reciprocamente, suponha que $\{x\} \notin p$ para todo $x \in X$. Para cada $x$, seja $q_{x}$ o ultrafiltro gerado por $\{x\}$. Fixe $x$. Temos que $p \neq q_{x}$, logo, existe $Z_{x} \in p$ zero-set com $Z_{x} \notin p$. Assim, $x \notin \bigcap p$. Como $x$ é arbitrário, $p$ é livre.

Daremos uma condição para que $\beta X \backslash X$ seja zero-dimensional.

Proposição 1.55. Seja $X$ um espaço de Tychonoff e suponha que não existe $f: X \rightarrow[0,1]$ sobrejetora e contínua. Então $\beta X$ é totalmente desconexo.

Demonstração. Basta ver que quaisquer dois pontos de $\beta X$ são separados por clopens.

Suponha que $p, q \in \beta X$ sejam $z$-ultrafiltros distintos. Então pelo item (c) da Proposição 1.44, existem $Z, W$ zero-sets não vazios de $X$ com $Z \in p, W \in q$ e $Z \cap W=\emptyset$. Pelo item (e) do Lema 1.39 , existe $f: X \rightarrow[0,1] \operatorname{com} Z=f^{-1}[\{0\}], W=f^{-1}[\{1\}]$. Temos que $f$ não é sobrejetora. Como 0,1 estão na imagem de $Z$, temos que existe $a \in(0,1)$ fora da imagem de $f$. Sejam $\tilde{Z}=f^{-1}[[0, a]]$, $\tilde{W}=f^{-1}[[a, 1]]$. Temos que $Z \subseteq \tilde{Z}, W \subseteq \tilde{W}, \tilde{Z} \cup \tilde{W}=X$ e $\tilde{Z} \cap \tilde{W}=\emptyset$. Pelo item (k) de 1.39 , temos que $\tilde{Z}, \tilde{W}$ são zero-sets. Pelo item $(d)$ da Proposição 1.51 , temos que $\operatorname{cl} e[\tilde{Z}] \cap \operatorname{cl} e[\tilde{W}]=\emptyset$. Temos que $\operatorname{cl} e[\tilde{Z}] \cup \operatorname{cl} e[\tilde{W}]=\beta X$. Logo, cl $e[\tilde{Z}]$ e cl $e[\tilde{W}]$ são clopens disjuntos de $\beta X$ e temos que $\tilde{Z} \in p, \tilde{W} \in q$, assim, $p \in \operatorname{cl} e[\tilde{Z}], q \in \operatorname{cl} e[\tilde{W}]$.

\subsection{Pseudocompacidade}

Definição 1.29. Um espaço $X$ é dito pseudocompacto se $C(X)=C^{*}(X)$, ou seja, se toda função contínua de $X$ em $\mathbb{R}$ é limitada.

Proposição 1.56. Seja $X$ um espaço de Tychonoff. São equivalentes:

a) $X$ é pseudocompacto.

b) Todo zero set não vazio de $\beta X$ intersecta $e[X]$.

c) Todo z-ultrafiltro de $X$ possui a propriedade da interseção enumerável.

d) Todo z-filtro de $X$ possui a propriedade da interseção enumerável.

Demonstração. $(a \rightarrow b)$ Suponha que $g: \beta X \rightarrow X$ é contínua, e suponha que $g^{-1}[\{0\}] \cap e[X]=\emptyset$. Seja $f=g \circ e$. Temos que $f$ nunca se anula, porém existe $p \in X^{*}$ com $g(p)=0$. Por continuidade, para todo $\epsilon>0$ existe $x \in X \operatorname{com}|f(x)|<\epsilon$, o que mostra que a função $\frac{1}{f}$ é contínua e ilimitada. Logo, $X$ não é pseudocompacto.

$(b \rightarrow a)$ Suponha que $X$ não é pseudocompacto. Então existe $f: X \rightarrow \mathbb{R}$ contínua e ilimitada. Temos que $|f|+1$ é uma função contínua, ilimitada e que não se anula em $X$. Então, sem perda de generalidade, podemos supor que $f$ não se anula em $X$. Seja $g$ contínua tal que $g \circ e=f$. Seja $h=\frac{1}{g}$. Temos que $0 \in \operatorname{cl} g[\beta X]$, como $\beta X$, é compacto, existe $p \in \beta X$ tal que $g(p)=0$. Logo, $g$ gera um zero-set não vazio que não intersecta $e[X]$.

$(b \rightarrow c)$ Seja $p$ um z-ultrafiltro sem a propriedade da interseção enumerável. Então existe uma sequência $A_{n}$ de zero sets de $X$ tais que $\bigcap_{n \in \omega} A_{n}=\emptyset$. Mas então $Z=\bigcap_{n \in \omega} W_{n}$, onde $W_{n}=$ $g_{n}^{-1}[\{0\}]$, onde $g_{n} \circ e=f_{n}$ e $g_{n}: \beta X \rightarrow \mathbb{R}$ é contínua, onde $f_{n}: \beta X \rightarrow[0,1]$ é contínua e $f_{n}^{-1}[\{0\}]=Z_{n}$, é um zero set que contém $p$ e não intersecta $e[X]$.

$(c \rightarrow b)$ Suponha que existe um zero set $Z$ de $\beta X$ que não intersecta $e[X]$ e tal que $p \in Z$. Temos que $z \in Z=\bigcap_{n \in \omega}$ cl $e\left[Z_{n}\right]$, onde $Z_{n}$ é um zero set de $X$. Para cada $n$, temos que $Z_{n} \in p$, e temos que $\bigcap_{n \in \omega} Z_{n}=\emptyset$, ou teríamos que $e[X] \cap Z \neq \emptyset$.

$(c \rightarrow d)$ A direção $\leftarrow$ é imediata. Para $\rightarrow$, se $p$ for um z-filtro sem a propriedade da interseção enumerável, ele se estende para um z-ultrafiltro sem essa propriedade. 
Corolário 1.57. Seja $X$ um espaço de Tychonoff. Temos que $X$ é compacto se, e somente se, $X$ é realcompacto e pseudocompacto.

Demonstração. Sabemos que $X$ é pseudocompacto se, e somente se, $\nu X=\beta X$, que $X$ é realcompacto se, e somente se, $e[X]=\nu X$ e que $X$ é compacto se, e somente se, $e[X]=\beta X$. Como vale que $e[X] \subseteq \nu X \subseteq \beta X$, segue a tese.

Proposição 1.58. Seja $X$ um espaço de Tychonoff. Então um zero set $Z \subseteq X$ é compacto se, e somente se, $Z$ não pertence à nenhum z-filtro livre. (um z-filtro livre é um z-filtro cuja interseção é vazia).

Demonstração. Suponha que $Z$ pertence à algum z-filtro livre. Então existe $p$ z-ultrafiltro tal que $z \in P$. Então, em $\beta X, p \in \operatorname{cl} e[Z]=e[Z]$, absurdo.

Reciprocamente, suponha que $Z$ não está em nenhum z-filtro livre. Queremos ver que $Z$ é compacto. Seja $\mathcal{B} \subseteq \mathscr{P}(Z)$ uma família de fechados com pif. Seja $\mathcal{F}$ a coleção de todos os zero sets que contém alguma interseção finita de elementos de $\mathcal{B}$. Temos que $\mathcal{F}$ é um $z$-filtro. Temos que $\bigcap \mathcal{B}=\bigcap \mathcal{F}$, pois se $x \in \bigcap \mathcal{B}$, então dado $A \in \mathcal{F}$, temos que $x \in A$ já que $\bigcap \mathcal{F} \subseteq K \subseteq A$, onde $K$ é uma interseção finita de elementos de $\mathcal{B}$. Reciprocamente, se $x \notin \bigcap \mathcal{B}$, existe $B \in \mathcal{B}$ com $x \notin B$, então existe $Z$ zero set com $x \notin W \supset B$. Temos que $W \in \mathcal{F}$, $\operatorname{logo}, x \notin \mathcal{W}$. Assim, segue a igualdade. Temos que $Z \in \mathcal{F}$, logo, $\mathcal{F}$ não é livre. Ou seja, $\mathcal{F}=\mathcal{B} \neq \emptyset$. Isso mostra que $Z$ é compacto.

Corolário 1.59. Todo zero-set $Z$ enumerável de um espaço de Tychonoff $X$ pseudocompacto é compacto.

Demonstração. Basta ver que $Z$ não está em nenhum ultrafiltro livre. Se $p$ é um ultrafiltro livre e $Z \in p$, dado $x \in Z$ temos que existe $A_{x} \in p$ zero-set com $x \notin A_{x}$. Assim, temos que $\bigcap_{x \in Z} A_{x} \cap Z=\emptyset$, o que viola o fato de que $p$ tem a propriedade da interseção enumerável.

\subsection{Forcing}

Utilizaremos a notação de [Kun80]. Uma classe $M$ é uma fórmula com uma variável livre. É comum pensar em uma classe como o "ente" que contém os elementos que satisfazem fórmula, mas como ZFC nem sempre admite a existência desses objetos, adotaremos a convenção de que uma classe é realmente uma fórmula. Porém, levando em conta este contexto, escrevemos $x \in M$ ao invés de $M(x)$. Se $M$ é uma classe ou um conjunto, define-se a relativização de uma fórmula $\phi$ à $M$. Na nossa abordagem, fórmulas serão elementos da metalinguagem. Assim, a definição abaixo, na realidade, é uma meta-definição.

Definição 1.30. Seja $M$. Define-se $\phi^{M}$ para toda fórmula $\phi$ por indução na complexidade da fórmula $\phi$, da seguinte forma:

- $(x=y)^{M}$ é $x=y$.

- $(x \in y)^{M}$ é $x \in y$.

- $(\phi \wedge \psi)^{M}$ é $\phi^{M} \wedge \psi^{M}$.

- $(\neg \phi)^{M}$ é $\neg\left(\phi^{M}\right)$.

- $(\exists x \phi)^{M}$ é $\exists x\left(x \in M \wedge \phi^{M}\right)$.

O seguinte resultado básico da lógica nos permite realizar provas de consistência. De acordo com a nossa abordagem, esse teorema é um meta-teorema, e não um teorema de ZFC. 
Lema 1.60. Suponha que $S, T$ são duas teorias de primeira ordem cuja linguagem é a da teoria dos conjuntos. Suponha que $T$ prova que $\exists x(x \in M)$ e que dado um axioma $\phi$ axioma de $S$, temos que $T$ prova $\phi^{M}$. Então se $T$ é consistente, $S$ é consistente.

$\mathrm{Na}$ metalinguagem, inserimos a seguinte definição:

Definição 1.31. Seja $\phi$ uma fórmula e $M$ um conjunto ou classe. E suponha que $x_{1}, \ldots, x_{n}$ são suas variáveis livres. Se $M \subseteq N$, dizemos que $\phi$ é absoluta para $M, N$ (com respeito a uma teoria $T)$ se:

$$
\forall x_{1}, \ldots, x_{n} \in M\left(\phi^{M}\left(x_{1}, \ldots, x_{n}\right) \leftrightarrow \phi^{N}\left(x_{1}, \ldots, x_{n}\right)\right)
$$

Se $M$ é um conjunto ou classe, $M \phi$ é absoluta para $M$ (com respeito a uma teoria $T$ ) se:

$$
\forall x_{1}, \ldots, x_{n} \in M\left(\phi^{M}\left(x_{1}, \ldots, x_{n}\right) \leftrightarrow \phi\left(x_{1}, \ldots, x_{n}\right)\right)
$$

Um símbolo de constante ou funcional (como $\omega, \cap$ ou $\emptyset$ ) é absoluto para $M$ (ou $M, N$ ) se a fórmula que o define é absoluta.

Lema 1.61. Suponha que $M$ seja um conjunto ou classe não vazio e transitivo tal que satisfaz cada um dos axiomas de ZFC (essa é uma meta hipótese, no nosso formalismo: Na meta teoria, dado um axioma $\phi$ ). Então, são absolutas para $M$ as fórmulas e simbolos funcionais:
1. $x \in y$
11. $x$ é transitivo.
21. $R$ é uma função
30. $A^{<\omega}$
2. $x=y$
12. Ux injetora.
3. $x \subseteq y$
13. $\bigcap x$
22. $x$ é um ordinal
4. $\{x, y\}$
14. $x$ é par ordenado.
23. $x$ é ord. limite.
5. $\{x\}$
15. $A \times B$
24. $x$ é ord. sucessor.
31. $R$ bem ordena $A$
6. $(x, y)$
16. $R$ é uma relação.
25. $x$ é ordinal finito.
32. type $(R)^{2}$.
7. $x \cup y$
17. $\operatorname{dom} R$
26. $\omega$
33. $\alpha+1$ ( $\alpha$ ordinal $)$
8. $x \cap y$
18. $\operatorname{ran} R$
27. $0,1,2, \ldots 20$
34. $\alpha-1^{3}$
9. $x \backslash y$
19. $R$ é uma função.
28. $x$ é finito
35. $\alpha+\beta$
10. $x \cup\{x\}$
20. $R(x)$
29. $A^{n}(n \in \omega)$
36. $\alpha . \beta$
37. $\alpha^{\beta}$ (exp. ordinal)

Precisaremos do Teorema da Reflexão.

Teorema 1.62. Seja $S$ uma teoria contendo os axiomas de ZFC. Então se $\phi_{1}, \ldots, \phi_{S}$ são axiomas de $S$, temos que $S$ prova que:

$$
\exists M\left(|M|=\omega \wedge M \text { é transitivo } \wedge \bigwedge_{i=1}^{n} \phi_{i}^{M}\right)
$$

\subsubsection{Adicionando uma Interpretação $M$}

Nessa seção, introduziremos a teoria ZFCM, ou, ainda, a teoria SM, sendo S uma teoria que estende ZFC.

\footnotetext{
${ }^{2}$ Tipo de boa ordem

${ }^{3} \alpha-1=\alpha$ se $\alpha$ não é sucessor
} 
Definição 1.32. Seja $S$ uma teoria que estende $Z F C$. Define-se $S M$ como sendo a teoria $S$ munida de um novo símbolo de constante $M$ cujos axiomas são:

- Os axiomas de $S$.

- $\phi^{M}$, onde $\phi$ é um axioma de $S$.

- $M$ é enumerável.

- $M$ é transitivo.

Teorema 1.63. Suponha que $S$ é uma teoria que estende $Z F C$. Então se $\phi$ é uma sentença da linguagem de $S$, temos que se $S M \vdash \phi$ (S prova $\phi$ ) então $S \vdash \phi$. Ou seja, $S M$ é uma extensão conservativa de $S$. Em particular, temos que as duas teorias são equiconsistentes.

Demonstração. Suponha que $S M \vdash \phi$. Pelo teorema da compacidade, sendo $\psi(M)$ a sentença que diz que $M$ é transitivo e enumerável, e sendo $T$ a teoria na linguagem de $S M$ cuja coleção dos axiomas é a mesma de $S$, temos que existem $\phi_{1}, \ldots, \phi_{n}$ axiomas de $Z F C$ tais que:

$$
T \cup\left\{\psi(M), \phi_{1}^{M}, \ldots, \phi_{n}^{M}\right\} \vdash \phi
$$

Pelo Teorema da Dedução, segue que:

$$
T \vdash \psi(M) \wedge \phi_{1}^{M} \wedge \cdots \wedge \phi_{n}^{M} \rightarrow \phi
$$

Sendo y uma variável não presente nas expressões acima, temos que:

$$
T \vdash \psi(y) \wedge \phi_{1}^{y} \wedge \cdots \wedge \phi_{n}^{y} \rightarrow \phi
$$

Logo:

$$
\begin{gathered}
S \vdash \psi(y) \wedge \phi_{1}^{y} \wedge \cdots \wedge \phi_{n}^{y} \rightarrow \phi \\
S \vdash \exists y\left(\psi(y) \wedge \phi_{1}^{y} \wedge \cdots \wedge \phi_{n}^{y}\right) \rightarrow \phi
\end{gathered}
$$

Pelo Teorema da Reflexão:

$$
S \vdash \exists y\left(\psi(y) \wedge \phi_{1}^{y} \wedge \cdots \wedge \phi_{n}^{y}\right)
$$

Logo:

$$
S \vdash \phi .
$$

\subsubsection{Forcing}

Definição 1.33. Um forcing poset é uma tripla $(P, \leq, 1)$, onde $\leq$ é uma pré-ordem em $P$ e 1 elemento máximo de $\leq$. É comum cometer o abuso de notação de chamar-se de forcing poset o conjunto $P$, deixando $\leq$ e 1 implícitos.

Sendo $M$ um conjunto, dizemos que $G \subseteq P$ é $P$-genérico sobre $M$ se $G$ é um filtro em $P$ tal que para todo $D \in M$ tal que $D \subseteq P$ e $D$ é denso, se $D \in M$ então $G \cap D \neq \emptyset$.

O lema abaixo nos garante a existência de genéricos para conjuntos enumeráveis.

Lema 1.64. Suponha que $M$ é enumerável e que $p \in P$. Existe $G P$-genérico sobre $M$ tal que $p \in G$. 
Demonstração. Seja $\left\{D_{n}: n \in \omega\right\}$ a coleção de todos os subconjuntos densos de $P$ que são elementos de $M$. Seja $p_{0}=p$. Definido $p_{n}$, seja $p_{n+1} \in D_{n}$ tal que $p_{n+1} \leq p_{n}$. Considere $G=\{q \in P: \exists n \in$ $\left.\omega\left(p_{n} \leq q\right)\right\}$.

A definição abaixo define, recursivamente, a noção de $P$-nome para um um forcing poset $P$.

Definição 1.34. Dizemos que $\tau$ é um $P$-nome se $\tau$ for uma coleção de pares ordenados tal que para todo $(\sigma, p) \in \tau, \sigma$ é um $P$-nome e $p \in P$.

Define-se $V^{P}$ como sendo a classe de todos os $P$-nomes. Se $M$ é classe ou conjunto, define-se $M^{P}=V^{P} \cap M$. Note que, se $M$ satisfazer cada um dos axiomas de ZFC, temos que $M^{P}=\{\tau \in$ $\left.M:(\tau \text { é um } P \text {-nome })^{M}\right\}$.

Também define-se recursivamente que $\operatorname{val}(\tau, G)=\tau_{G}=\{\operatorname{val}(\sigma, G): \exists p \in P((\sigma, p) \in \tau \wedge p \in G)\}$. Temos que essa definição é absoluta para interpretações transitivas de ZFC.

Define-se que $M[G]=\left\{\tau_{G}: \tau \in M^{P}\right\}$.

Define-se recursivamente (usando a relação $\in$ ) para $x$ qualquer, o $P$-nome $\check{x}=\{(\check{y}, 1): y \in x\}$. Finalmente, define-se $\Gamma=\{(\check{p}, p): p \in P\}$.

Teorema 1.65. Na notação anterior, vale que, se $M$ é uma interpretação transitiva e enumerável de ZFC e $G$ é $P$-genérico sobre $M$ :

- $M[G]$ é uma interpretação transitiva e enumerável de ZFC tal que $M \subseteq M[G]$ e $G \in M[G]$.

- Se $x \in M$, então $\check{x} \in M^{P}$ e $\check{x}_{G}=x$.

- $\Gamma_{G}=G$.

- Se $N$ é uma interpretação transitiva e enumerável de $Z F C$ tal que $M \subseteq N$ e $G \in N$, então $M[G] \subseteq N$.

Definição 1.35. Na notação anterior, $P$ é uma noção de Forcing não trivial sobre $M$ se, e somente se, para todo $G P$-genérico sobre $M, G \notin M$.

Lema 1.66. Na notação anterior, suponha que:

$$
\forall p \in P \exists q, r \in P(q \leq p \wedge r \leq p \wedge q \perp r)
$$

então $P$ é uma noção de Forcing não trivial sobre $M$.

Definição 1.36. Seja $\phi\left(x_{1}, \ldots, \phi_{n}\right)$ uma fórmula tal que todas as suas variáveis livres são alguma das $x_{1}, \ldots, x_{n}$. Seja $M$ uma interpretação transitiva e enumerável de ZFC, $P$ um forcing poset em $M$ e $\tau_{1}, \ldots, \tau_{n} \in M^{P}$ e $p \in P$. Define-se que:

$$
p \Vdash_{P, M} \phi\left(\tau_{1}, \ldots, \tau_{n}\right)
$$

se, e somente se, para todo $G P$-genérico sobre $M$ tal que $p \in G$, temos que:

$$
\phi^{M[G]}\left(\tau_{1 G}, \ldots, \tau_{n G}\right)
$$

É comum omitir $P, M$ quando estes estão claros.

Definição 1.37. Seja $\phi\left(x_{1}, \ldots, \phi_{n}\right)$ uma fórmula tal que todas as suas variáveis livres são alguma das $x_{1}, \ldots, x_{n}$. Seja $P$ um forcing poset e $\tau_{1}, \ldots, \tau_{n} \in V^{P}$ e $p \in P$. Define-se recursivamente:

$$
p \Vdash^{*} \phi\left(\tau_{1}, \ldots, \tau_{n}\right)
$$

da seguinte forma: 
(a) $p \Vdash^{*} \tau_{1}=\tau_{2}$ se, e somente se: Para todo $\left(\pi_{1}, s_{1}\right) \in \tau_{1}$,

$$
\left\{p \leq q: q \leq s_{1} \rightarrow \exists\left(\pi_{2}, s_{2} \in \tau_{2}\left(q \leq s_{2} \wedge q \Vdash^{*} \pi_{1}=\pi_{2}\right)\right\}\right.
$$

é denso sob $p$, e para todo $\left(\pi_{2}, s_{2}\right) \in \tau_{2}$

$$
\left\{p \leq q: q \leq s_{2} \rightarrow \exists\left(\pi_{1}, s_{1} \in \tau_{1}\left(q \leq s_{1} \wedge q \Vdash^{*} \pi_{1}=\pi_{2}\right)\right\}\right.
$$

é denso sob $p$.

(b) $p \Vdash^{*} \tau_{1} \in \tau_{2}$ se, e somente se:

$$
\left\{q: \exists(\pi, s) \in \tau_{2}\left(q \leq s \wedge q \Vdash^{*} \pi=\tau_{1}\right)\right\}
$$

é denso sob $p$.

(c) $p \Vdash^{*}\left(\phi\left(\tau_{1}, \ldots, \tau_{n}\right) \wedge \psi\left(\tau_{1}, \ldots, \tau_{n}\right)\right.$ se, e somente se:

$$
p \Vdash^{*} \phi\left(\tau_{1}, \ldots, \tau_{n}\right) \wedge p \Vdash^{*} \psi\left(\tau_{1}, \ldots, \tau_{n}\right)
$$

(d) $p \Vdash^{*} \neg \phi\left(\tau_{1}, \ldots, \tau_{n}\right)$ se, e somente se, não existe $q \leq p$ tal que:

$$
q \Vdash^{*} \phi\left(\tau_{1}, \ldots, \tau_{n}\right)
$$

(e) $p \Vdash^{*} \exists x \phi\left(x, \tau_{1}, \ldots, \tau_{n}\right)$ se, e somente se

$$
\left\{r: \exists \sigma \in V^{P}\left(r \Vdash^{*} \phi\left(\sigma, \tau_{1}, \ldots, \tau_{n}\right)\right)\right\}
$$

é denso sob $p$.

Teorema 1.67. Seja $M$ uma interpretação transitiva e enumerável de ZFC. Seja $P$ um forcing poset em $M$. Seja $\phi\left(x_{1}, \ldots, x_{n}\right)$ uma fórmula com todas as variáveis livres aparecendo e $\tau_{1}, \ldots, \tau_{n} \in M^{P}$. Então para todo $p \in P$ :

$$
p \Vdash \phi\left(\tau_{1}, \ldots, \tau_{n}\right) \leftrightarrow\left(p \Vdash^{*} \phi\left(\tau_{1}, \ldots, \tau_{n}\right)\right)^{M} .
$$

E, para todo $G$ que é $P$-genérico sobre $M$ :

$$
\phi\left(\tau_{1 G}, \ldots, \tau_{n G}\right)^{M[G]} \leftrightarrow \exists p \in G\left(p \Vdash \phi\left(\tau_{1}, \ldots, \tau_{n}\right)\right) .
$$

Alguns teoremas importantes sobre forcing:

Proposição 1.68. Se $P$ tem c.c.c., então $P$ preserva cofinalidades, ou seja, cf é uma função absoluta para $M, M[G]$. Isso implica que $P$ preserva cardinalidades.

Definição 1.38. Sejam $Q, P$ forcing posets e $i: Q \rightarrow P$. Dizemos que $i$ é uma imersão completa se:

1. $\forall p, p^{\prime} \in P\left(p^{\prime} \leq p \rightarrow i\left(p^{\prime}\right) \leq i(p)\right)$.

2. $\forall p_{1}, p_{2} \in P\left(p_{1} \perp p_{2} \leftrightarrow i\left(p_{1}\right) \perp i\left(p_{2}\right)\right)$

3. $\forall q \in Q \exists p \in P\left(\forall p^{\prime} \in P\left(p^{\prime} \leq p \rightarrow\left(i\left(p^{\prime}\right)\right.\right.\right.$ e $q$ são compatíveis em $\left.\left.Q\right)\right)$. Dado $q \in Q$, um tal $p$ é chamado de redução de $Q$. 
4. $i\left(1_{P}\right)=1_{Q}$

Define-se recursivamente na classe dos $P$-nomes:

Sejam $Q, P$ forcing posets e $i: Q \rightarrow P$. Dizemos que $i$ é uma imersão densa se:

1. $\forall p, p^{\prime} \in P\left(p^{\prime} \leq p \rightarrow i\left(p^{\prime}\right) \leq i(p)\right)$.

2. $\forall p_{1}, p_{2} \in P\left(p_{1} \perp p_{2} \rightarrow i\left(p_{1}\right) \perp i\left(p_{2}\right)\right)$

3. $i[P]$ é denso em $Q$.

4. $i\left(1_{P}\right)=1_{Q}$

$$
i_{*}(\tau)=\left\{\left(i_{*}(\sigma), i(p)\right):(\sigma, p) \in \tau\right\}
$$

Verifica-se recursivamente que $i_{*}(\tau)$ é um $Q$-nome e que essas definições são absolutas para interpretações transitivas de ZFC.

Note que todo isomorfismo é uma imersão densa.

Proposição 1.69. Sejam $i, P, Q \in M$, sendo $P, Q$ forcing posets, $i: P \rightarrow Q$ imersão completa e $M$ uma interpretação transitiva e enumerável de ZFC. Seja $H$ um filtro $Q$-genérico sobre $M$. Então:

1. $i^{-1}[H]$ é $P$-genérico sobre $M$ e $M\left[i^{-1}[H]\right] \subseteq M[H]$.

2. Se $\tau \in M^{P}$, então $\tau_{i^{-1}[H]}=i_{*}(\tau)_{H}$.

3. Se $\phi\left(x_{1}, \ldots, x_{n}\right)$ é uma fórmula absoluta para interpretações transitivas de ZFC. Sejam $\tau_{1}, \ldots, \tau_{n} \in M^{P}$ e $p \in P$. Então:

$$
p \Vdash_{P} \phi\left(\tau_{1}, \ldots, \tau_{n}\right) \leftrightarrow i(p) \Vdash_{Q} \phi\left(i_{*}\left(\tau_{1}\right), \ldots, i_{*}\left(\tau_{n}\right)\right)
$$

Além disso, toda imersão densa é completa, e caso a imersão seja densa, a igualdade vale em 1. e 3. vale para qualquer fórmula. Além disso, é verdade que se $i$ é uma imersão densa, as extensões genéricas geradas por $P$ e por $Q$ são exatamente as mesmas, assim, $P$ e $Q$ são equivalentes com respeito à Forcing.

Definição 1.39. Seja $P$ um forcing poset e $\sigma$ um $P$-nome. Um $P$-nome $\tau$ é um nice name para um subconjunto de $\sigma$ se $\tau=\bigcup\left\{\{\pi\} \times A_{\pi}: \pi \in \operatorname{dom} \sigma\right\}$, onde cada $A_{\pi}$ é uma anticadeia em $P$.

Proposição 1.70. Seja $M$ uma interpretação transitiva de ZFC, $P \in M$ um forcing poset, $\sigma, \mu \in$ $M^{P}$. Então existe um nice name $\tau \in M^{P}$ para um subconjunto de $\sigma$ tal que:

$$
1 \Vdash \mu \subseteq \sigma \rightarrow \mu=\tau .
$$

Proposição 1.71. Seja $M$ uma interpretação transitiva e enumerável de ZFC e sejam $P, Q \in M$ forcing posets. Seja $P \times Q$ o forcing poset ordenado coordenada-a-coordenada. Então $P \times Q \in M$. Sejam $G \subseteq P, H \subseteq Q$. São equivalentes:

1. $G \times H$ é $P \times Q$-genérico sobre $M$.

2. $G$ é $P$-genérico sobre $M$ e $H$ é $Q$-genérico sobre $M[G]$.

3. $H$ é $Q$-genérico sobre $M$ e $G$ é $P$-genérico sobre $M[H]$.

Além disso, se essas cláusulas são verdadeiras, então $M[G \times H]=M[G][H]=M[H][G]$. 


\section{Capítulo 2}

\section{Almost disjoint families e $\Psi$-espaços}

Neste capítulo iremos inserir as definições mais básicas sobre $\Psi$-espaços e provaremos alguns fatos sobre elas. Nós nos baseamos primariamente na notação de [Hru14].

$\Psi$-espaços, também conhecidos como Espaços de Mrówka, foram inventados por S. Mrówka e seu uso já se fazia presente em artigos como [Mro70].

\subsection{Almost disjoint families}

Começaremos com definições básicas:

Definição 2.1. Seja $X$ um conjunto infinito. Dizemos que uma coleção infinita $\mathcal{A} \subseteq \mathscr{P}(X)$ é uma almost disjoint family em $X$ se $\forall A \in \mathcal{A}(|A|=|X|)$ e $\forall A, B \in \mathcal{A}(A \neq B \rightarrow|A \cap B|<|X|)$.

Dizemos que uma almost family $\mathcal{A}$ em $X$ é maximal ( $\mathcal{A}$ é uma MAD family em $X$ ) se não existe uma almost disjoint family $\mathcal{B}$ em $X$ que a contém propriamente.

Uma almost disjoint family, quando não especificada um conjunto, é uma almost disjoint family em $\omega$.

Nesse documento, trabalharemos primariamente com almost disjoint families em $\omega$.

Notemos que se $X$ é infinito enumerável, então uma almost disjoint family em $X$ é uma coleção infinita de subconjuntos infinitos de $X$ tal que, dois a dois, seus elementos possuem interseção finita.

Há noções ligeiramente distintas para o termo almost disjoint family. Por exemplo, há autores que permitem que almost disjoint families sejam finitas (por exemplo, [Kun80]). Neste texto decidimos optar por permitir apenas almost disjoint families infinitas a fim de evitar algumas patologias. Além disso, vale a pena observar que apesar de termos definido almost disjoint families para um conjunto arbitrário, há textos em que se exige que eles sejam enumeráveis. A grande maioria do uso e dos exemplos deste objeto combinatório neste texto será em algum conjunto enumerável.

Almost disjoint families podem ser encaradas como generalizações naturais de coleções disjuntas infinitas de subconjuntos infinitos. Dado um conjunto $X$ infinito, sempre é possível particionar $X$ em $|X|$ conjuntos de cardinalidade $|X|$, logo, almost disjoint families sempre existem. A proposição abaixo, portanto, nos garante a existência de MAD families.

Proposição 2.1. Seja $X$ um conjunto infinito. Então toda almost disjoint family $\mathcal{A}$ em $X$ está contida em alguma MAD family em $X$.

Demonstração. Seja $\mathcal{F}$ a coleção de todas as almost disjoint families em $X$ que contém $\mathcal{A}$. $\mathcal{F}$ é parcialmente ordenado pela relação $\subseteq$, e não vazio, pois $\mathcal{A} \in \mathcal{F}$. Se $C$ é uma cadeia não vazia de almost disjoint families que contém $\mathcal{A}$, então $\bigcup C$ também é uma almost disjoint family em $X$ que contém $A$, logo, toda cadeia é limitada superiormente. Pelo Lema de Zorn, existe um elemento $\mathcal{M}$ de $\mathcal{F}$ que é maximal. Pela definição de $\mathcal{F}$, temos que $\mathcal{A} \subseteq \mathcal{M}$ e esta última é uma almost disjoint family em $X$. Resta ver que é maximal. Se não for, existe $\mathcal{B} \neq \mathcal{M}$ almost disjoint family em $X$ tal que $\mathcal{A} \subseteq \mathcal{M} \subseteq \mathcal{B}$. Então $\mathcal{B} \in \mathcal{F}$, mas isso viola a maximalidade em $\mathcal{F}$ de $\mathcal{M}$. 
Podemos provar algo ligeiramente diferente que é útil para realizar diversas construções: se temos uma almost disjoint family (em $\omega$ ) de cardinalidade $\mathfrak{c}$, podemos "engordar" alguns dos conjuntos a fim de obter uma MAD family. Lembremos que, quando não escrevemos qual o conjunto base da almost disjoint families em questão, estamos nos referindo a almost disjoint families em $\omega$. Porém, é claro que, tomando bijeções, as propriedades combinatóricas de almost disjoint families sobre $\omega$ podem ser provadas também para almost disjoint families sobre qualquer conjunto infinito enumerável.

Lema 2.2. Seja $\mathcal{A}$ uma almost disjoint family de cardinalidade $\mathfrak{c}$. Então existe uma MAD family $\mathfrak{B}=\left\{B_{A}: A \in \mathcal{A}\right\}$ tal que se $A, A^{\prime} \in \mathcal{A}$ e $A \neq A^{\prime}$ então $B_{A} \neq B_{A^{\prime}}$, e tal que para todo $A \in \mathcal{A}$, $A \subset B_{A}$.

Demonstração. Seja $\mathcal{C}$ uma MAD family contendo $\mathcal{A}$. Seja $u: \mathcal{C} \backslash \mathcal{A} \rightarrow \mathcal{A}$ injetora. Seja

$$
\mathcal{B}=(\mathcal{A} \backslash u[\mathcal{C} \backslash \mathcal{A}]) \cup\{A \cup u(A): A \in \mathcal{C} \backslash \mathcal{A}\}
$$

Temos que $\mathcal{B}$ é uma MAD family de cardinalidade c. Se $A \in \mathcal{A} \backslash u[\mathcal{C} \backslash \mathcal{A}]$, seja $B_{A}=A$. Se $A \in \mathcal{C} \backslash \mathcal{A}$, seja $B_{A}=A \cup u(A)$. Assim, $\mathcal{B}=\left\{B_{A}: A \in \mathcal{A}\right\}$ é como no enunciado.

Apesar do teorema acima dizer coisas sobre almost disjoint families de cardinalidade $\mathfrak{c}$, ainda não sabemos que um objeto dessa forma existe.

Teorema 2.3. Existe uma almost disjoint family de cardinalidade $\mathfrak{c}$.

Apresentaremos duas demonstrações para o teorema acima.

Demonstração. Para todo $x \in \mathbb{R} \backslash \mathbb{Q}$, seja $x^{\prime}$ uma sequência injetora de racionais que converge para $x$, e seja $A_{x}=\operatorname{ran} x^{\prime}$ (a imagem da sequência). Dados $x, y$ irracionais distintos, como $\mathbb{R}$ é Hausdorff temos que $A_{x} \cap A_{y}$ é finito. Para cada $x$, temos que $A_{x} \subseteq \mathbb{Q}$. Assim, temos que $\left\{A_{x}: x \in \mathbb{R} \backslash \mathbb{Q}\right\}$ é uma almost disjoint family em $\mathbb{Q}$ de cardinalidade $\mathfrak{c}$. Tomando uma bijeção entre $\mathbb{Q}$ e $\omega$, segue a tese.

Para uma segunda demonstração, provaremos uma versão mais geral do teorema acima.

Teorema 2.4. Seja $\kappa$ um cardinal infinito tal que $2^{<\kappa}=\kappa$. Existe uma almost disjoint family sobre $\kappa$ de cardinalidade $2^{\kappa}$.

Demonstração. Como $2^{<\kappa}=\kappa$, é suficiente mostrar que existe uma almost disjoint family sobre o conjunto de funções $<\kappa 2$ de cardinalidade $\kappa$. Se $f \in{ }^{\kappa} 2$, seja $A_{f}=\{f \mid \alpha: \alpha<\kappa\}$. Se $f \neq g$ temos que $\left|A_{f} \cap A_{g}\right|<\kappa$, logo, $\left\{A_{f}: f \in{ }^{\kappa} 2\right\}$ é uma almost disjoint family como desejávamos.

Note que exemplos de cardinais não enumeráveis que satisfazem a hipótese acima são os cardinais $\beth_{\alpha}$ para $\alpha$ limite. Lembremos que define-se $\beth_{0}=\omega$. Definido $\beth_{\alpha}$, define-se $\beth_{\alpha+1}=2^{\beth_{\alpha}}$, e, se $\alpha$ é limite, então $\beth_{\alpha}=\sup \left\{\beth_{\beta}: \beta<\alpha\right\}$. Agora fixe um ordinal limite $\alpha$. Está claro que $\beth_{\alpha}=\sup \left\{\beth_{\beta}\right.$ : $\beta<\alpha\} \leq \sup \left\{2^{\kappa}: \kappa<\beth_{\alpha}\right\} \leq 2^{<\beth_{\alpha}}$. Para a inclusão contrária, se $\lambda<\beth_{\alpha}$, pela definição de $\beth_{\alpha}$ existe $\beta<\alpha$ com $\lambda \leq \beth_{\beta}$. Logo, $2^{\lambda} \leq 2^{\beth_{\beta}}=\beth_{\beta+1} \leq \beth_{\alpha}$, o que prova a outra desigualdade.

Agora, discutiremos um pouco mais acerca da cardinalidade das MAD families. Veremos a seguir que toda MAD family é não enumerável. Este fato decorre do teorema abaixo para $\kappa=\omega$.

Proposição 2.5. Seja $\kappa$ um cardinal regular. Então toda MAD family em $\kappa$ possui cardinalidade diferente de $\kappa$.

Demonstração. Suponha que $\mathcal{A}=\left\{A_{\alpha}: \alpha \in \kappa\right\}$ é uma almost disjoint family em $\kappa$. Veremos que ela não é maximal. Podemos supor que se $\alpha \neq \beta$ então $A_{\alpha} \neq A_{\beta}$. Assim, se $\alpha \neq \beta$, temos que $\left|A_{\alpha} \cap A_{\beta}\right|<\kappa$. Para cada $\alpha<\kappa$, seja $b_{\alpha}=\min \left(A_{\alpha} \backslash \bigcup_{\beta<\alpha}\left(A_{\alpha} \cap A_{\beta}\right)\right)$. Note que essa união é um conjunto de cardinalidade $<\kappa$ pois $\kappa$ é regular, logo $b_{\alpha}$ está bem definido. Seja $B=\left\{b_{\alpha}: \alpha<\kappa\right\}$. Temos que $|B|=\kappa$. Para ver isso, veremos que se $\alpha \neq \beta$, então $b_{\alpha} \neq b_{\beta}$. Com efeito, dados $\alpha, \beta$ 
distintos, sem perda de generalidade podemos supor $\alpha<\beta$. Então, por definição, $b_{\alpha} \in A_{\alpha}$, porém, $b_{\beta} \notin A_{\alpha}$, pois caso contrário teríamos que $b_{\beta} \in A_{\alpha} \cap A_{\beta}$, o que é absurdo pela definição de $b_{\beta}$. Temos também que para todo $\alpha,\left|B \cap A_{\alpha}\right|<\kappa$, pois $B \cap A_{\alpha} \subseteq\left\{b_{\beta}: \beta \leq \alpha\right\}$ já que se $\beta>\alpha$ temos que $b_{\beta} \notin A_{\alpha}$. Dessa forma, $\mathcal{A} \cup\{B\}$ é uma almost disjoint family que contém $\mathcal{A}$ propriamente. Logo, $\mathcal{A}$ não é maximal.

Observação: Não é possível inferir que toda MAD family em $\kappa$ possui cardinalidade maior que $\kappa$. Com efeito, seja $\mathcal{A}=\left\{A_{n}: n \in \omega\right\}$ uma partição de $\omega_{1}$ de modo que para todo $n \in \omega,\left|A_{n}\right|=\omega_{1}$. Temos que $\mathcal{A}$ é uma MAD family, pois se $A \in\left[\omega_{1}\right]^{\omega_{1}}$, existe $n$ tal que $\left|A \cap A_{n}\right|=\omega_{1}$.

Corolário 2.6. Seja $\kappa$ um cardinal regular. Então existe uma MAD family em $\kappa$ de cardinalidade $\geq \kappa^{+}$.

Demonstração. Seja $\mathcal{A}$ uma partição de $\kappa$ em $\kappa$ subconjuntos de $\kappa$ de cardinalidade $\kappa$. Existe uma MAD family estendendo $\mathcal{A}$. Pela proposição anterior, segue que ela tem cardinalidade $\geq \kappa^{+}$.

Sabemos que existe uma MAD family (em $\omega$ ) de cardinalidade $\mathfrak{c}$, que é a maior cardinalidade possível para uma MAD family já que $|\mathscr{P}(\omega)|=\mathfrak{c}$. Uma pergunta natural é: o quão pequena uma MAD family pode ser? Pela proposição anterior, qualquer MAD family é não enumerável. Define-se $\mathfrak{a}$ como sendo o menor cardinal para o qual existe uma MAD family desta cardinalidad. Segue que $\omega_{1} \leq \mathfrak{a} \leq \mathfrak{c}$.

Por fim, podemos definir uma notação para o ideal livre gerado por uma almost disjoint family. Falaremos dele ao discutirmos sobre happy families, mais adiante.

Definição 2.2. Dada uma almost disjoint family $\mathcal{A}$, o ideal livre gerado por $\mathcal{A}$ é $\mathcal{I}(\mathcal{A})=\{X \subseteq$ $\left.\omega: \exists B \in[\mathcal{A}]^{<\omega}\left(X \subseteq^{*} \bigcup B\right)\right\}$, e a coleção dos conjuntos positivos com relação a ele é $\mathcal{I}^{+}(\mathcal{A})=$ $\mathscr{P}(\omega) \backslash \mathcal{I}(\mathcal{A})$.

Se $X \in \mathcal{I}^{+}(\mathcal{A})$, dizemos que $X$ é $\mathcal{A}$-positivo.

\subsection{Pequenos Cardinais}

Nessa seção, iremos definir e provar algumas propriedades de alguns dos chamados pequenos cardinais. Seguimos a notação de [Kun11]. Dentre eles, temos o cardinal a que está diretamente relacionado com o assunto desta dissertação.

Definição 2.3. O cardinal a é a menor cardinalidade possível para uma MAD family. Vimos na Proposição 2.5 que $\omega<\mathfrak{a} \leq \mathfrak{c}$.

O cardinal $\mathfrak{d}$ é a cardinalidade da menor família dominante de $\omega^{\omega}$, ou seja, é a menor cardinalidade possível para uma coleção $A$ de funções de $\omega$ em $\omega$ tal que $\forall f \in \omega^{\omega} \exists g \in A f \leq^{*} g$.

O cardinal $\mathfrak{b}$ é a cardinalidade da menor família ilimitada, ou seja, a cardinalidade da menor coleção $\mathcal{B} \subseteq \omega^{\omega}$ tal que $\neg \exists g \in \omega^{\omega} \forall f \in \mathcal{B} f \leq^{*} g$.

Lembremos que se $f, g: X \rightarrow \omega$, dizemos que $f \leq^{*} g$ se, e somente se, existe $X^{\prime} \subseteq X$ finito tal que se $x \in X \backslash X^{\prime}$ então $f(x) \leq g(x)$. De forma análoga, define-se $f<^{*} g$.

Os valores destes cardinais são independentes de ZFC, porém as relações entre eles são bem estudadas. Abaixo, mencionaremos algumas.

Proposição 2.7. Na notação acima:

(a) Toda família dominante de $\omega^{\omega}$ é ilimitada.

(b) $\mathfrak{b} \leq \mathfrak{d} \leq \mathfrak{c}$

(c) $\omega<\mathfrak{b}$

(d) $\mathfrak{b} \leq \mathfrak{a}$. 
Demonstração. Dada uma família dominante $\mathcal{D}$, suponha por absurdo que ela não seja ilimitada. Então existe $g: \omega \rightarrow \omega$ tal que para toda $f \in \mathcal{D}$ temos que $f \leq^{*} g$. Seja $h(n)=g(n)+1$. Temos que dada $f \in \omega^{\omega}, h \mathbb{Z}^{*} f$, visto que $\{n \in \omega: h(n)>f(n)\} \supset\{n \in \omega: g(n) \geq f(n)\}$, que é cofinito. Isso prova (a). (b) decorre diretamente de (a) e do fato de que $\omega^{\omega}$ é uma família ilimitada.

Para (c), seja $\mathcal{B}=\left\{f_{n}: n \in \omega\right\}$ uma coleção infinita enumerável de funções. Seja $g(k)=$ $\max \left\{f_{n}(k): n \leq k\right\}$. Temos que para cada $n, f_{n} \leq^{*} g$.

Para (d), fixe $\kappa<\mathfrak{b}$. Veremos que $\kappa<\mathfrak{a}$. Seja $\mathcal{D}=\left\{X_{\alpha}: \alpha<\kappa\right\}$ almost disjoint family. Veremos que $\mathcal{D}$ não é maximal. Para cada $n \in \omega$, seja $\hat{X}_{n}=X_{n} \backslash \bigcup_{i<n} X_{i}$. Segue que cada $\hat{X}_{n}$ é infinito, que $\hat{X}_{n}={ }^{*} X_{n}$ e que se $n \neq m, \hat{X}_{n} \cap \hat{X}_{m}=\emptyset$. Dado $g \in \omega^{\omega}$, seja $e_{g}^{n}=\min \left\{k \in \hat{X}_{n}: k \geq g(n)\right\}$ e $E_{g}=\left\{e_{g}^{n}: n \in \omega\right\}$. Para cada $n,\left|E_{g} \cap \hat{X}_{n}\right|=1$. Logo, $E_{g}$ é infinito, e, para cada $n,\left|E_{g} \cap X_{n}\right|<\omega$. Agora, para cada $\alpha$ tal que $\omega \leq \alpha<\kappa$, como $X_{\alpha} \cap X_{n}$ é finito para todo $n$, tomemos $f_{\alpha}$ tal que para cada $n, f_{\alpha}(n)>\max X_{\alpha} \cap X_{n}$. Como $\kappa<\mathfrak{b}$, existe $g$ tal que $f_{\alpha} \leq^{*} g$ para todo $\alpha$. Então $E_{g} \cap X_{\alpha}$ para todo $\omega \leq \alpha<\kappa$, pois se $f_{\alpha}(n) \leq g(n)$ então $e_{g}^{n} \notin X_{\alpha}$.

Proposição 2.8. $\mathfrak{b}$ é regular e $\operatorname{cf} \mathfrak{d} \geq \mathfrak{b}$.

Demonstração. Vejamos que $\mathfrak{b}$ é regular. Seja $\left\{f_{\alpha}: \alpha<\mathfrak{b}\right\}$ uma família ilimitada de cardinalidade $\mathfrak{b}$. Para cada $\alpha<\mathfrak{b}$, recursivamente, seja $g_{\alpha}$ tal que $g_{\alpha} \geq g_{\beta}$ para todo $\beta \leq \alpha$, de modo que $g_{\alpha} \geq f_{\alpha}$ o que é possível pela definição de $\mathfrak{b}$ pois $\left|\left\{g_{\alpha}: \alpha<\mathfrak{b}\right\}\right|<\mathfrak{b}$. Assim, temos que $\left\{g_{\alpha}: \alpha<\mathfrak{b}\right\}$ é uma família ilimitada tal que se $\alpha \leq \beta<\mathfrak{b}$ então $g_{\alpha} \leq^{*} g_{\beta}$. Seja $u:$ cf $\mathfrak{b} \rightarrow \mathfrak{b}$ cofinal. Afirmo que $\left\{g_{u(\alpha)}: \alpha \in \operatorname{cf} \mathfrak{b}\right\}$ é ilimitada.

Com efeito, se $h \geq^{*} g_{u(\alpha)}$ para todo $\alpha \in \mathrm{cf} \mathfrak{b}$, temos que dado $\beta<\mathfrak{b}$, existe $\alpha \in$ cf $\mathfrak{b}$ tal que $\beta \leq u(\alpha)$, então segue que $g_{\beta} \leq^{*} g_{u(\alpha)} \leq^{*} h$. Mas, então $h$ majora $\left\{g_{\alpha}: \alpha<\mathfrak{b}\right\}$, o que é absurdo.

Portanto, $\left\{g_{u(\alpha)}: \alpha \in \operatorname{cf} \mathfrak{b}\right\}$ é ilimitada. Mas isso implica que $\mathrm{cf} \mathfrak{b} \geq \mathfrak{b}$, e, portanto, vale a igualdade.

Para a segunda desigualdade, seja $\mathfrak{D}$ uma família dominante de cardinalidade $\mathfrak{d}$. Podemos particionar $\mathfrak{D}$ em uma coleção $\left\{D_{\alpha}: \alpha<\operatorname{cf} \mathfrak{d}\right\}$ tal que $\left|D_{\alpha}\right|<\mathfrak{d}$ para todo $\alpha<\operatorname{cf} \mathfrak{d}$. Para cada $\alpha$, seja $f_{\alpha}$ tal que não existe $g \in D_{\alpha} \operatorname{com} f_{\alpha} \leq^{*} g$. Afirmo que $\left\{f_{\alpha}: \alpha<\operatorname{cf} \mathfrak{c}\right\}$ é ilimitada.

Com efeito, se $h \geq^{*} f_{\alpha}$ para todo $\alpha$, tome $g \in \mathcal{D}$ tal que $g \geq^{*} h$. Existe $\alpha$ tal que $g \in D_{\alpha}$. Mas então $g \geq^{*} h \geq^{*} f_{\alpha}$, o que é absurdo.

Logo, existe uma família ilimitada de cardinalidade $\operatorname{cf} \mathfrak{d}$, o que implica $\mathfrak{b} \leq \operatorname{cf} \mathfrak{d}$.

Notemos que então, vale que $\omega_{1} \leq \operatorname{cf} \mathfrak{b}=\mathfrak{b} \leq \operatorname{cf} \mathfrak{d} \leq \mathfrak{d} \leq \mathfrak{c}$. É verdade que é possível criar um modelo onde $\mathfrak{b}, \mathfrak{d}$ e $\mathfrak{c}$ podem ser quaisquer cardinais respeitando essa relação (desde que cf $\mathfrak{c}>\omega$ ). Para uma referência, ver o Teorema 2.4 de [Bla10]. A prova completa deste teorema, com todas as proposições preliminares, foge do escopo desta dissertação. Porém, não utilizaremos este fato, mas apenas alguns mais fracos. No Apêndice B, provaremos que é consistente que $\omega_{1}=\mathfrak{b}<\mathfrak{d}=\mathfrak{c}$, pois isso traz consequências que serão utilizadas.

Introduziremos agora o número de pseudointerseção.

Definição 2.4. Uma coleção $A$ de conjuntos tem a propriedade da interseção finita forte se $\bigcap F$ é infinito para todo $F \subseteq A$ finito não vazio. Uma pseudo-interseção de $A$ é um conjunto $K$ infinito tal que $K \subseteq^{*} Z$ para todo $Z \in A$.

Lema 2.9. Seja $A$ uma coleção enumerável de conjuntos com a propriedade da interseção finita forte. Então $A$ possui uma pseudo-interseção.

Demonstração. Seja $\left\{A_{n}: n \in \omega\right\}=A$. Recursivamente, escolhemos $k_{0} \in A_{0}$. Definidos $k_{0}, \ldots, k_{n}$, escolhemos $k_{n+1}>k_{n}$ com $k_{n+1} \in \bigcap_{i<n} A_{i}$. Considere $K=\left\{k_{n}: n \in \omega\right\}$.

Definição 2.5. $\mathfrak{p}$ é o primeiro cardinal para o qual existe $A \subseteq[\omega]^{\omega} \operatorname{com}|A|=\mathfrak{p}$ com a propriedade da interseção finita forte sem pseudo-interseção.

Provaremos a seguir que $\mathfrak{p} \leq \mathfrak{b}$. Para isso, utilizaremos o lema abaixo. 
Lema 2.10. Seja $\mathcal{A} \subseteq[\omega]^{\omega}$ com a propriedade da interseção finita forte $\operatorname{com}|\mathcal{A}|<\mathfrak{p}$. Seja $\mathcal{H} \subseteq[\omega]^{\omega}$ com $|H|<\mathfrak{p}$. Suponha que para todo $H \in \mathcal{H},\{Z \cap H: Z \in \mathcal{A}\}$ tem a propriedade da interseção finita forte. Então $\mathcal{A}$ tem uma pseudo-interseção $K$ tal que $K \cap H$ é infinita para todo $H \in \mathcal{H}$.

Demonstração. Seja $J=[\omega]^{<\omega} \backslash\{\emptyset\}$. Para $Z \in \mathcal{A}$. Seja $\hat{Z}=\{s \in J: s \in Z\}$. Para $H \in \mathcal{H}$, seja $\tilde{H}=\{s \in J: s \cap H \neq \emptyset\}$. Para $l \in \omega$, seja $T_{l}=\{s \in J: \min (s)>l\}$. Seja

$$
\mathcal{A}^{*}=\{\hat{Z}: Z \in \mathcal{A}\} \cup\{\tilde{H}: H \in \mathcal{H}\} \cup\left\{T_{l}: l \in \omega\right\} \subseteq \mathscr{P}(J)
$$

Temos que $\left|\mathcal{A}^{*}\right|<\mathfrak{p}$. Temos que $\mathcal{A}^{*}$ tem a propriedade da interseção finita forte: ados $\hat{Z}_{1} \ldots, \hat{Z}_{m}, \tilde{H}_{1} \ldots, \tilde{H}_{n}$ e $T_{l}$, temos que para cada $i$, existe $x_{i} \in Z_{1} \cap \cdots \cap Z_{m} \cap H_{i} \operatorname{com} x_{i}>l$. Seja $s=\left\{x_{1}, \ldots, x_{n}\right\}$. Temos que se $s \subseteq s^{\prime} \in\left[Z_{1} \cap \cdots \cap Z_{m} \backslash(l+1)\right]^{<\omega}$, então $s^{\prime} \in \mathcal{A}^{*}=\{\hat{Z}: Z \in \mathcal{A}\} \cup\{\tilde{H}: H \in \mathcal{H}\} \cup\left\{T_{l}: l \in \omega\right\}$.

Como $J$ é enumerável e $\left|\mathcal{A}^{*}\right|<\mathfrak{p}$, existe $M \subseteq J$ infinito pseudointerseção de $\mathcal{A}^{*}$. Seja $K=\bigcup M$. Como $M$ é infinito e $M \subseteq \mathscr{P}(K)$, temos que $K$ é infinito. Para cada $Z \in \mathcal{A}$, como $M \subseteq^{*} \hat{Z}$, temos que $K \subseteq^{*} Z$.

Dado $H \in \mathcal{H}$, fixe $l \in \omega$. Temos que $M \subseteq^{*} \tilde{H} \cap T_{l}$. Fixe $s \in M$ com $s \in \tilde{H} \cap T_{l}$. Temos que $s \cap H \neq \emptyset$ e min $s>l$, logo $s \cap H \subseteq \bigcup M=K$ implica que $K \cap H$ contém um número maior que $l$. Como $l$ é arbitrário, segue a tese.

Teorema 2.11. $\omega_{1} \leq \mathfrak{p} \leq \mathfrak{b} \leq \mathfrak{a} \leq \mathfrak{c}$ e $\omega_{1} \leq \mathfrak{p} \leq \mathfrak{b} \leq \mathfrak{d} \leq \mathfrak{c}$

Demonstração. Resta provar apenas que $\mathfrak{p} \leq \mathfrak{b}$. Fixe $k<\mathfrak{p}$. Veremos que $\kappa<\mathfrak{b}$. Seja $\mathcal{B} \subseteq \omega^{\omega}$ de cardinalidade $\kappa$. Veremos que $\mathcal{B}$ não é ilimitada.

Para cada $f \in \mathcal{B}$, seja $Z_{f}=\{(x, y) \in \omega \times \omega: y>f(x)\}$. Seja $\mathcal{A}=\left\{Z_{f}: f \in \mathcal{B}\right\}$. Para $J \in \omega$, seja $H_{j}=\{j\} \times \omega$, e seja $\mathcal{H}=\left\{H_{j}: j \in \omega\right\}$. Fixado $j$, temos que $\left\{Z_{f} \cap H_{j}: f \in \mathcal{A}\right\}$ tem a propriedade da interseção forte. Pelo lema anterior aplicado a $\omega \times \omega$, existe $K \subseteq \omega \times \omega$ tal que $K$ é pseudointerseção de $\mathcal{A}$ e tal que para cada $j, K \cap H_{j}$ é infinito. Tomemos $g \subseteq K$ que é gráfico de função. Então, para cada $f \in \mathcal{B}$, temos que $g \subseteq^{*} Z_{f}$, o que imlica $f \leq^{*} g$.

Para complementar a seção, definiremos alguns cardinais a partir de um ideal.

Definição 2.6. Seja $\mathcal{I}$ um ideal próprio em um conjunto $X$. Define-se:

(a) $\operatorname{add}(\mathcal{I})$ é o menor cardinal $\kappa$ para o qual existe $\mathcal{C} \in[\mathcal{I}]^{\kappa} \operatorname{com} \bigcup \mathcal{C} \notin \mathcal{I}$.

(b) $\operatorname{non}(\mathcal{I})$ é o menor cardinal $\kappa$ para o qual existe $A \in[X]^{\kappa} \operatorname{com} A \notin \mathcal{I}$.

Vejamos algumas desigualdades famosas envolvendo alguns ideais. Primeiro, vamos lembrar o Teorema de Baire.

Definição 2.7. Seja $X$ um espaço topológico. Dizemos que $A \subseteq X$ é de primeira categoria se $A$ pode ser escrito como $\bigcup_{n \in \omega} A_{n}$, onde int $\mathrm{cl} A_{n}=\emptyset$. É imediato que a coleção de todos os conjuntos de primeira categoria é um ideal, e que se $X$ é Hausdorff sem pontos isolados, é livre.

Teorema 2.12 (Baire). Seja $X$ um espaço Hausdorff localmente compacto. Seja $\left(U_{n}\right)_{n \in \omega}$ uma sequência de abertos densos. Temos que $\bigcap_{n \in \omega} U_{n}$ é denso.

Demonstração. Fixe $A \subseteq X$ aberto. Como $A \cap U_{0}$ é um aberto não vazio, existe $V_{0}$ aberto não vazio com cl $V_{0} \subseteq A \cap U_{0}$ e cl $V_{0}$ compacto. Construído $V_{n}$ aberto não vazio, seja $V_{n+1}$ aberto não vazio com cl $V_{n+1} \subseteq V_{n} \cap J_{n+1}$. Temos que $\operatorname{cl} V_{0} \supset \operatorname{cl} V_{1} \supset \ldots$, dessa forma, pela compacidade de $\mathrm{cl} V_{0}$, temos que $\bigcap_{n \in \omega} \operatorname{cl} V_{n} \neq \emptyset$. Porém, $\bigcap_{n \in \omega} \operatorname{cl} V_{n} \subseteq A \cap \bigcap_{n \in \omega} U_{n}$.

Corolário 2.13. Seja $\mathcal{M}$ o ideal dos conjuntos de primeira categoria em $\mathbb{R}$. Então $\mathcal{M}$ é um ideal próprio livre e $\operatorname{add}(\mathcal{M}) \geq \omega_{1}$. 
Demonstração. Está claro que $\mathcal{M}$ é um ideal livre. Mostraremos que $\mathbb{R}$ não é de primeira categoria, o que mostra que ele é próprio. Se o fosse, existiria $A_{n}$ sequência de conjuntos cujo fecho tem interior vazio e tal que $\mathbb{R}=\bigcup_{n \in \omega} A_{n}$. Seja $B_{n}=\mathbb{R} \backslash A_{n}$. Temos que cada $\operatorname{int} B_{n}$ é denso e $\bigcap_{n \in \omega} \operatorname{int} B_{n}=\emptyset$, o que viola o teorema de Baire.

Lema 2.14. $\mathfrak{b} \leq \operatorname{non}(\mathcal{M})$, onde $\mathcal{M}$ é o ideal dos conjuntos de primeira categoria em $\mathbb{R}$

Demonstração. Suponha que $\kappa<\mathfrak{b}$. Veremos que $\kappa<\operatorname{non}(\mathcal{M})$. Para isso, fixe $X \subseteq \mathbb{R}$ de cardinalidade $\kappa$. Mostraremos que $X$ é disjunto de uma interseção densa de abertos com interior vazio. Enumeremos $\mathbb{Q}=\left\{q_{k}: k \in \omega\right\}$. Para cada $f \in \omega^{\omega}$, seja $U_{f}^{n}=\bigcup\left\{\left(q_{k}-2^{-f(k)}, q_{k}+2^{-f(k)}\right): k \geq n\right\}$. Temos que $U_{f}^{n}$ é aberto, denso e fixado $f, U_{f}^{n}$ forma uma sequência estritamente decrescente. Seja $G_{f}=\bigcap_{n \in \omega} U_{f}^{n}$. Veremos que $X \cap G_{g}=\emptyset$ para algum $g$.

Notemos que $f \leq^{*} g \rightarrow G_{g} \subseteq G_{f}$, pois $U_{g}^{n} \subseteq U_{f}^{n}$ para todos a menos de uma quantidade finita de $n$ 's. Para cada $x \in X$, escolha alguns $f_{x} \in \omega^{\omega}$ tal que $x \notin G_{f_{x}}$. Isso é possível pois podemos tomar $f$ tal que $2^{-f_{x}(k)}<\left|x-q_{k}\right|$ para cada $k$ com $q_{k} \neq x$. Como $|X|<\mathfrak{b}$, tome $g$ com $f_{x} \leq^{*} g$ para todo $x \in X$. Então $x \notin G_{g}$ para todo $x \in X$.

Na Proposição 1.13, aplicamos o Axioma de Martin com uma ordem parcial que possui uma propriedade especial: Ela é a uma ordem $\sigma$-centrada, $\operatorname{assim}, \operatorname{MA}_{P}(\kappa)$ vale para todo $\kappa<\mathfrak{p}$ (ver Apêndice II). Assim, segue que:

Proposição 2.15. $\kappa<\mathfrak{p}$ implica que para todo $\mathcal{A}, \mathcal{C} \subseteq[\omega]^{<\kappa}$ tais que para todo $y \in \mathcal{C}$ e para todo $F \subseteq \mathcal{A}$ finito, $|y \backslash \bigcup F|=\omega$, existe $D \subseteq \omega$ tal que $\forall x \in \mathcal{A}(|D \cap x|<\omega)$ e $\forall Y \in \mathcal{C}(|D \cap Y|=\omega)$.

A proposição abaixo é uma aplicação interessante de almost disjoint families.

Proposição 2.16. Se $\omega \leq \kappa<\mathfrak{p}$, então $2^{\omega}=2^{\kappa}$.

Demonstração. Tome uma almost disjoint family $\mathcal{B}$ de cardinalidade $\kappa$. Seja $\phi: \mathscr{P}(\omega) \rightarrow \mathscr{P}(\mathcal{B})$ dada por $\phi(D)=\{X \in \mathcal{B}:|D \cap X|<\omega\}$. Veremos que $\phi$ é sobrejetora.

Considere $\mathcal{A} \subseteq \mathcal{B}$ arbitrária. Na Proposição 2.15 , seja $\mathcal{C}=\mathcal{B} \backslash \mathcal{A}$. temos que $\mathcal{A}$ e $\mathcal{C}$ satisfazem as hipóteses, logo, existe $D$ tal que $\phi(D)=\{X \in \mathcal{B}:|D \cap X|<\omega\}=\mathcal{A}$.

Assim, segue que $2^{\kappa}=|\mathscr{P}(\mathcal{B})| \geq|\mathscr{P}(\omega)|=2^{\omega}$.

Corolário 2.17. $\mathrm{cf} \mathfrak{c} \geq \mathfrak{p}$

Demonstração. Da proposição acima e do lema de König, segue que $\operatorname{cf}\left(2^{\omega}\right)=\operatorname{cf}\left(2^{\kappa}\right)>\kappa$ para todo $\kappa \operatorname{com} \kappa<\mathfrak{p}$. Logo, $\operatorname{cf}\left(2^{\omega}\right) \geq \mathfrak{p}$.

Teorema 2.18. $\mathfrak{m} \leq \mathfrak{p}$.

Demonstração. Fixe $\kappa<\mathfrak{m}$. Veremos que $\kappa<\mathfrak{p}$. Suponha que $\mathcal{A}$ é uma família com a propriedade da interseção forte de cardinalidade $\kappa$.

Considere $P=[\omega]^{<\omega} \times[\mathcal{A}]^{<\omega}$ ordenado por:

$$
\left(s^{\prime}, F^{\prime}\right) \leq(s, F) \leftrightarrow s \subseteq s^{\prime} \wedge F \subseteq F^{\prime} \wedge \forall x \in F\left(s^{\prime} \backslash s \subseteq x\right)
$$

É fácil verificar que $\leq$ é uma pré-ordem.

Notemos que se $(s, F)$ e $\left(s, F^{\prime}\right)$ forem elementos de $P$ com $s=s^{\prime}$, então $\left(s, F \cup F^{\prime}\right)$ é extensão comum. Logo, como $[\omega]^{<\omega}$ é enumerável, segue que toda anticadeia em $P$ é enumerável. Logo, $P$ tem c.c.c.

Para cada $n$ natural, seja $D_{n}=\{(s, F) \in P:|s| \geq n\}$. Temos que $D_{n}$ é denso: dado $(s, F) \in P$, seja $s^{\prime} \subseteq \bigcap F$ de cardinalidade $n$ e considere $\left(s \cup s^{\prime}, F\right)$.

Para cada $X \in \mathcal{A}$, seja $E_{X}=\{(s, F) \in P: x \in F\}$, visto que dado $(s, F) \in P$ temos que $(s, F \cup\{X\}) \subseteq(s, F)$. Seja $G$ um filtro em $P$ que intersecta cada $E_{x}, D_{n}$. Seja $K=\bigcup\{s:(s, F) \in$ $F\}$. Dado $n$, temos que existe $(s, F) \in G \cap D_{n}$, logo, para esse $s$, temos que $|K| \geq|s| \geq n$. Logo, $K$ é infinito. Tome $X \in \mathcal{A}$. Temos que ver que $K \backslash X$ é finito. Tomemos $(s, F) \in G \operatorname{com} X \in F$. 
Veremos que $K \backslash s \subseteq X$. De fato, dado $\left(s^{\prime}, F^{\prime}\right) \in G$, existe $\left(s^{\prime \prime}, F^{\prime \prime}\right) \in G$ extensão comum de $(s, F)$ e $\left(s^{\prime}, F^{\prime}\right)$. Assim, temos que $s^{\prime \prime} \backslash s \subseteq X$. Isso mostra que para todo $\left(s^{\prime}, F^{\prime}\right) \in G$ temos que $s^{\prime \prime} \backslash s \subseteq X$. Logo, $K \backslash s \subseteq X$.

\section{$2.3 \Psi$-espaços}

Com uma almost disjoint family $\mathcal{A}$, podemos definir um espaço topológico bastante especial, o espaço $\Psi(\mathcal{A})$, conhecido como também como espaço de Isbell-Mrówka. Também é comum chama-los simplesmente de " $\Psi$-espaços". Veremos que trabalhando propriedades combinatórias em $\mathcal{A}$, obtemos propriedades topológicas em $\Psi(\mathcal{A})$. Tais construções são bastante úteis para responder perguntas em Topologia Geral, e tais perguntas nem sempre estão ligadas inicialmente a almost disjoint families.

Definição 2.8. Dada uma almost disjoint family $\mathcal{A}$, define-se $\Psi(\mathcal{A})=\omega \cup \mathcal{A}$ com a topologia gerada por $\mathcal{B}=\{\{A\} \cup(A \backslash n): A \in \mathcal{A}, n \in \omega\} \cup\{\{n\}: n \in \omega\}$.

Ou seja, estamos pedindo que $\omega$ seja aberto discreto e que $A$ seja uma sequência convergente para o elemento $A$.

O lema abaixo é de fácil demonstração e sua verificação fica a cargo do leitor.

Lema 2.19. Na notação acima, $\mathcal{B}$ é base para a topologia que gera, e uma base local para $A \in \mathcal{A}$ é $\{\{A\} \cup(A \backslash n) \in \omega\}$. Assim, $\omega$ é discreto e denso em $\Psi(\mathcal{A})$, e esse espaço é first-countable.

Observação: Às vezes, é útil trabalhar com almost disjoint families em um conjunto $X$ infinito enumerável diferente de $\omega$. Neste caso, supondo-se $[X]^{\omega} \cap X=\emptyset^{1}$, pode-se definir $\Psi(\mathcal{A})=\mathcal{A} \cup X$, e tomar como topologia a topologia gerada pela base $\mathcal{B}=\{\{A\} \cup(A \backslash F): A \in \mathcal{A}, F \in[X]<\omega\} \cup\{\{x\}$ : $x \in X\}$. Sendo $f: X \rightarrow \omega$ bijeção, é fácil ver que a $\mathcal{B}=\{f[A]: A \in \mathcal{X}\}$ é uma almost disjoint family em $\omega$ e que $F: \Psi(\mathcal{A}) \rightarrow \Psi(\mathcal{B})$ dado por $F(A)=f[A]$, para $A \in \mathcal{A}$, e $F(x)=f(x)$, para $x \in X$, é um homeomorfismo. Assim, podemos nos restringir a estudar $\Psi$-espaços gerados por almost disjoint families de $\omega$, mas, quando conveniente, podemos aplicar os resultados desenvolvidos para $\Psi$-espaços de almost disjoint families de algum $X$ infinito enumerável, e utiliza-los para provar teorema sobre $\Psi$-espaços.

Proposição 2.20. Seja $\mathcal{A}$ uma almost disjoint family. Temos que $\Psi(\mathcal{A})$ é Hausdorff e que $\mathcal{B}=$ $\{\{A\} \cup(A \backslash n): A \in \mathcal{A}, n \in \omega\} \cup\{\{n\}: n \in \omega\}$ é uma base de compactos. Assim, $\Psi(\mathcal{A})$ é localmente compacto e zero dimensional.

Demonstração. Dado $A \in \mathcal{A}$ e $n \in \omega$, temos que $\{n\},\{A\} \cup(A \backslash(n+1))$ são abertos disjuntos. Se $n, m$ são naturais distintos, $\{n\}$ e $\{m\}$ são abertos disjuntos. Finalmente, se $A, B \in \mathcal{A}$ são distintos, sendo $n>\max A \cap B$, temos que $\{A\} \cup(A \backslash n)$ e $\{B\} \cup(B \backslash n)$ são abertos disjuntos. Assim, o espaço é Hausdorff.

Assim, temos que para todo $n,\{n\}$ é clopen (e compacto). Fixe $n, A$. Suponha que $\mathcal{B}$ é uma cobertura de abertos básicos para $\{A\} \cup(A \backslash n)$. Algum dos abertos da cobertura possui $A$ como elemento, logo, existe $m \in \omega \operatorname{com}\{A\} \cup(A \backslash m) \in \mathcal{B}$. Como $[\{A\} \cup(A \backslash n)] \backslash[\{A\} \cup(A \backslash m)]$ é finito, segue a tese.

Um $\Psi$-espaço nunca é compacto, como veremos na proposição abaixo.

Proposição 2.21. Seja $\mathcal{A}$ uma almost disjoint family. Então $\Psi(\mathcal{A})$ não é compacto.

Demonstração. Para cada $A \in \mathcal{A}$, seja $V_{A}=\{A\} \cup \omega$. Temos que $\left\{V_{A}: A \in \mathcal{A}\right\}$ é uma cobertura aberta de $\Psi(\mathcal{A})$ sem subcobertura finita, pois qualquer subconjunto finito dessa cobertura recobre apenas uma quantidade finita de elementos de $\mathcal{A}$.

\footnotetext{
${ }^{1}$ Esta condição é necessária para que a união $\mathcal{A} \cup X$ seja disjunta.
} 
Nesse texto, exigimos que almost disjoint families sejam infinitas. Se estivéssemos permitindo que almost disjoint families possam ser finitas, então poderiam existir $\Psi$-espaços compactos. Por exemplo, $\mathcal{A}=\{\omega\}$ daria origem à uma compactificação por um ponto de $\omega$.

A pseudocompacidade de $\Psi(\mathscr{A})$ está relacionada apenas com a maximalidade de $\mathscr{A}$.

Proposição 2.22. Seja $\mathcal{A}$ uma almost disjoint family. Temos que $\Psi(\mathcal{A})$ é pseudocompacto se, e somente se, $\mathcal{A}$ for uma MAD family.

Demonstração. Suponha que $\mathcal{A}$ é MAD family. Seja $f: \Psi(\mathcal{A}) \rightarrow \mathbb{R}$ contínua. Veremos que $f[\omega]$ é limitado. Suponha por absurdo que $f[\omega]$ é ilimitado para cima. Recursivamente, podemos construir uma sequência estritamente crescente de números naturais $n_{k}$ tais que $f\left(n_{k}\right)+1<f\left(n_{k+1}\right)$. Seja $B=$ $\left\{n_{k}: k \in \omega\right\}$. Temos que $|B|=\omega$. Fixe $A \in \mathcal{A}$. Afirmo que $|A \cap B|<\omega$, que viola a maximalidade de $\mathcal{A}$. Para ver isso, note que $f$ é contínua em $A$. Daí, existe $n$ natural com $f[A \backslash n] \subseteq\{f(A)\}$. Mas então, a sequência dos $f\left(n_{k}\right)$ é limitada, o que é absurdo. Assim, $f[\omega]$ é limitado superiormente. Analogamente, é limitado inferiormente. Por fim, por continuidade, $f[\Psi(\mathcal{A})]=f[\bar{\omega}] \subseteq \operatorname{cl} f[\omega]$, que é limitado.

Agora suponha que $\mathcal{A}$ não é maximal. Existe $B \subseteq \omega$ infinito tal que $B \cap A$ é finito para todo $A \in \mathscr{A}$. Sabemos que $B$ é aberto. Vejamos que $B$ é fechado: suponha que $p \in \bar{B}$. Suponha por absurdo que $p \notin B$. Temos que $p$ é um ponto de acumulação de $B$. Como $\Psi(\mathcal{A})$ é Hausdorff, devemos ter que se $V$ é aberto e $p \in V$ então $V \cap B$ é infinito. Mas isso não ocorre: se $p \in \omega$, tome $V=\{p\}$. Caso contrário, temos $p \in \mathscr{A}$, daí, tome $V=\{p\} \cup p$. Então $B$ é clopen. Escrevemos injetivamente $B=\left\{b_{n}: n \in \omega\right\}$. Considere a função $f: \Psi(\mathcal{A}) \rightarrow \mathbb{R}$ dada por $f(x)=n$, se $x=b_{n}$, e $f(x)=0$ caso contrário. Como $B$ é clopen e discreto, essa função é contínua e ilimitada.

$\mathrm{Na}$ verdade, vale algo ainda mais forte do que isso. Veremos que se $\mathcal{A}$ é uma MAD family, então $(\Psi(\mathcal{A}))^{\omega}$ é pseudocompacto. Mas antes, precisaremos do seguinte lema que pode ser encontrado no artigo [HHR07].

Definição 2.9. Seja $X$ um espaço topológico e $A \subseteq X$. Dizemos que $A$ é relativamente sequencialmente compacto (com relação à $X$ ) se toda sequência em $A$ possui uma subsequência que converge para um ponto de $X$. Se $X$ é T1, dizemos que $A$ é relativamente enumeravelmente compacto se todo subconjunto infinito de $A$ possui um ponto de acumulação em $X$.

Lema 2.23. Seja $X$ um espaço topológico e $D \subseteq X$ um subconjunto denso relativamente sequencialmente compacto. Então $X$ é pseudocompacto.

Demonstração. Suponha por absurdo que exista $f: X \rightarrow \mathbb{R}$ contínua e ilimitada. Sem perda de generalidade, $f$ é ilimitada para cima. Assim, dado $n$ natural, existe $d_{n} \in D$ tal que $d_{n} \in$ $f^{-1}[(n, \infty)]$, ou seja, tal que $f\left(d_{n}\right) \geq n$. Existe $x \in X$ e $\phi: \omega \rightarrow \omega$ estritamente crescente tal que $d_{\phi(m)} \rightarrow x$ quando $m \rightarrow \infty$. Mas então temos que $f\left(d_{\phi(m)}\right) \rightarrow \infty$ e $f\left(d_{\phi(m)}\right) \rightarrow x$ quando $m \rightarrow \infty$, o que é absurdo.

Proposição 2.24. Suponha que para cada $n, A_{n} \subseteq X_{n}$ é relativamente sequencialmente compacto em $X_{n}$. Então $A=\prod_{n \in \omega} A_{n}$ é relativamente sequencialmente compacto em $X=\prod_{x \in \omega} X_{n}$.

Demonstração. Para cada $m$ natural, seja $f_{m} \in \prod_{n \in \omega} A_{m}$. Devemos ver que $\left(f_{m}\right)_{m \in \omega}$ possui uma subsequência que converge para algum elemento de $X$. Existem $g(0) \in X_{0}$ e $A_{0} \subseteq \omega$ infinito tal que $f_{m}(0) \mid A_{0} \rightarrow x_{0}$. Recursivamente, define-se $A_{n+1} \subseteq A_{n}$ infinito e $g(n+1) \in X_{n+1}$ tal que $f_{m}(n+1) \mid A_{n+1} \rightarrow x_{n+1}$. Seja $A$ uma pseudointerseção da família $A_{n} \operatorname{com} n \in \omega$. Temos que para todo $n$, a função de variável $m$ dada por $f_{m}(n) \mid A \rightarrow g(n)$, logo $\left(f_{m}\right)_{m \in \omega} \mid A \rightarrow g$.

Notemos que, colocando $A_{n}=X_{n}$ na proposição acima, obtemos o famoso resultado da preservação de compacidade sequencial para produtos enumeráveis. Essa proposição nos permite obter alguns corolários interessantes.

Corolário 2.25. Suponha que para cada $n, A_{n} \subseteq X_{n}$ é um denso relativamente sequencialmente compacto em $X_{n}$. Então $X=\prod_{x \in \omega} X_{n}$ é pseudocompacto. 
Demonstração. Temos que $A=\prod_{n \in \omega} A_{n}$ é relativamente sequencialmente compacto à $X$ e é denso, o que implica que $X$ é pseudocompacto.

Proposição 2.26. Seja $\mathcal{A}$ uma almost disjoint family em $\omega$. Temos que $\omega$ é relativamente sequencialmente compacto em $\Psi(\mathcal{A})$ se, e somente se, $\mathcal{A}$ é MAD.

Demonstração. Suponha que $\mathcal{A}$ seja MAD e seja $\phi: \omega \rightarrow \omega$ uma sequência injetora. Temos que existe $B \in \mathcal{A}$ tal que $\phi[\omega] \cap B$ é infinito. Segue que $\phi \mid \phi^{-1}[B] \rightarrow B$ em $\Psi(\mathcal{A})$.

Reciprocamente, suponha que $\mathcal{A}$ não é MAD e seja $B \subseteq \omega$ infinito tal que $B \cap A$ é finito para todo $A \in \mathcal{A}$. Seja $\phi: \omega \rightarrow B$ bijeção estritamente crescente. $\phi$ não converge para nenhum ponto de $\omega$ pois este é discreto, e $\phi$ não converge para nenhum ponto $A \in \mathcal{A}$ pois a imagem de $\phi$ intersectada $\operatorname{com} A$ é finita.

Assim, sintetizando os resultados acima, temos que:

Corolário 2.27. Seja $\mathcal{A}_{n}(n \in \omega)$ uma família enumerável de almost disjoint families. São equivalentes:

a) Para todo $n \in \omega, \mathcal{A}_{n}$ é MAD.

b) $\omega^{\omega} \subseteq \prod_{n \in \omega} \Psi\left(\mathcal{A}_{n}\right)$ é relativamente sequencialmente compacto.

c) $\prod_{n \in \omega} \Psi\left(\mathcal{A}_{n}\right)$ é pseudocompacto.

Em particular, $\mathcal{A}$ é uma MAD family se, e somente se, $(\psi(\mathcal{A}))^{\omega}$ é pseudocompacto.

Podemos verificar que a classe dos $\Psi$-espaços é fechada por somas diretas finitas.

Proposição 2.28. Sejam $\mathcal{A}, \mathcal{B}$ almost disjoint families. Então existe uma almost disjoint family $\mathcal{C}$ tal que:

$$
\Psi(\mathcal{A}) \oplus \Psi(\mathcal{B})=\Psi(\mathcal{C})
$$

Demonstração. Utilizemos a notação de que $A \oplus B=\{(a, 0): a \in A\} \cup\{(b, 1): b \in B\}$.

Seja $\mathcal{C}=\{2 A: A \in \mathcal{A}\} \cup\{2 B+1: B \in \mathcal{B}\}$. Temos que $\mathcal{C}$ é uma almost disjoint family.

Define-se $f: \Psi(\mathcal{A}) \oplus \Psi(\mathcal{B}) \rightarrow \Psi(\mathcal{C})$ dada por $f(A, 0)=2 A, f(B, 1)=2 B+1, f(n, i)=2 n+i$ para $n \in \omega, A \in \mathcal{A}$ e $B \in \mathcal{B}$. Fica a cargo do leitor verificar que $f$ é homeomorfismo.

Lema 2.29. Seja $\mathcal{A}$ uma almost disjoint family e $\sim$ uma relação de equivalência em $\mathcal{A}$ tal que cada classe de equivalência seja finita. Estendemos para $\psi(\mathcal{A})$ definindo em $\omega$ que $n \sim m$ se, e somente se, $n=m$. Então existe uma almost disjoint family $\mathcal{B}$ tal que $\Psi(\mathcal{B}) \approx \Psi(\mathcal{A}) / \sim$, considerando a topologia quociente.

Demonstração. Basta definir $\mathcal{B}=\left\{\bigcup[A]_{\sim}: A \in \mathcal{A}\right\}$. Temos que $\mathcal{B}$ é uma almost disjoint family. Seja $\phi: \Psi(\mathcal{B}) \rightarrow \Psi(\mathcal{A}) / \sim$ dada por $\phi([n])=n$ para todo $n \in \omega$, e $\phi([A])=\bigcup[A]$ para todo $A \in \mathcal{A}$. Fica a cargo do leitor verificar que $\phi$ é um homeomorfismo.

Nosso objetivo agora é mostrar que existem $2^{\mathfrak{c}}$ MAD families que geram espaços dois a dois não homeomorfos seguindo ideias de [Mro77].

Lema 2.30. Suponha que $\mathcal{A}, \mathcal{B}$ são almost disjoint families e $T: \Psi(\mathcal{A}) \rightarrow \Psi(\mathcal{B})$ homeomorfismo. Então $T[\mathcal{A}]=\mathcal{B}, T[\omega]=\omega$ para todo $A \in \mathcal{A}$, a diferença simétrica $T(A) \Delta T[A]$ é finita.

Demonstração. $T[\omega]=\omega$ pois homeomorfismos bijetam pontos isolados. Assim, $T[\mathcal{A}]=\mathcal{B}$. Se $T[A] \backslash T(A)$ for infinito, seja $\left(a_{n}\right)_{n \in \omega}$ sequência em $A$ com $\left\{T\left(a_{n}\right): n \in \omega\right\} \subseteq T[A] \backslash T(A)$. Temos que $a_{n} \rightarrow A$ mas $T\left(a_{n}\right) \not \rightarrow T(A)$, absurdo. Agora suponha que $T(A) \backslash T[A]$ é infinito. Seja $\left(a_{n}\right)_{n \in \omega}$ sequência disjunta de $A \operatorname{com}\left\{T\left(a_{n}\right): n \in \omega\right\} \subseteq T(A) \backslash T[A]$. Temos que $T\left(a_{n}\right) \rightarrow T(A)$ nas $a_{n} \not \rightarrow A$, absurdo. 
Proposição 2.31. Sejam $P, Q \subseteq \omega$ infinitos disjuntos com $P \cup Q=\omega$. Sejam $\mathcal{A}$ uma MAD family no conjunto $P$ e $\mathcal{B}$ uma MAD family no conjunto $Q$, ambas de mesma cardinalidade $\kappa$ (ou seja, $\mathcal{A}, \mathcal{B}$ são almost disjoint family de subconjuntos de $P, Q$ maximais nesse sentido). Seja $F$ o conjunto de todas as funções bijetoras de domínio $\mathcal{A}$ e imagem $\mathcal{B}$. Para cada $f \in F$, seja $\mathcal{A}_{f}=\{A \cup f(A): f \in F\}$. Então:

a) para cada $f \in F, \mathcal{A}_{f}$ é uma MAD family em $\omega$ e cardinalidade $\kappa$,

b) se $f, g \in \mathcal{F}$ e $f \neq g$, então $\mathcal{A}_{f} \neq \mathcal{A}_{g}$, e $\left|\left\{\mathcal{A}_{f}: f \in F\right\}\right|=2^{\kappa}$,

c) se $f, g \in F$ e $\phi$ é um homeomorfismo de $\Psi\left(\mathcal{A}_{f}\right)$ em $\Psi\left(\mathcal{A}_{g}\right)$ que fixa $\omega$, então $f=g$ e $\phi$ é a identidade,

d) se $\mathcal{C}$ é uma almost disjoint family, então $\left|\left\{f \in F: \Psi(\mathcal{C}) \approx \Psi\left(\mathcal{A}_{f}\right)\right\}\right| \leq 2^{\omega}$.

Demonstração. Na notação do enunciado:

a) Fixe $f$. Sejam $A, B \in \mathcal{A}$ e suponha que $A \cup f(A) \neq B \cup f(B)$. Então $A \neq B$ e $f(A) \neq f(B)$. Como $P \cap Q=\emptyset$, temos que $(A \cup f(A)) \cap(B \cup f(B))=(A \cap B) \cup(f(A) \cap f(B))$, que é finito. Além disso, a função $A \rightarrow A \cup f(A)$ é bijetora. Assim, $\mathcal{A}_{f}$ é infinito de cardinalidade $\kappa$. Segue que $\mathcal{A}_{f}$ é almost disjoint. Para ver que é MAD, fixe $X \subseteq \omega$ infinito. Temos que ao menos um dos $X \cap P, X \cap Q$ é infinito. Caso o primeiro seja, então existe $A \in \mathcal{A}$ com $X \cap P \cap A=X \cap A$ infinito. Logo, $X \cap(A \cup f(A))$ é infinito. Para o segundo caso, existe $A \in \mathcal{A}$ com $f(A) \cap X \cap Q=f(A) \cap Q$ infinito. Logo, $(A \cup f(A)) \cap Q$ é infinito. Segue a tese.

b) Existe $A$ tal que $f(A) \neq g(A)$. Então $f(A) \cup A \notin \mathcal{A}_{g}$, pois se $B \neq A$ e $g(B) \neq f(A)$, temos que $(A \cup f(A)) \cap(B \cup g(B))$ é finito, se $B=A$ e $g(B) \neq f(A)$ temos que $(A \cup$ $f(A)) \Delta(B \cup g(B))=f(A) \Delta g(B)$, que é infinito, e, por último, $B \neq A$ e $g(B)=f(A)$ temos que $(A \cup f(A)) \Delta(B \cup g(B))=A \Delta B$, que é infinito. A segunda parte desse item decorre do Lema 1.8 .

c) Tome $A \in \mathcal{A}$ qualquer. Existe $B \in \mathcal{A}$ com $\phi(A \cup f(A))=B \cup g(B)$. Note que $\phi(A \cup$ $f(A)) \Delta \phi[A \cup f(A)]$ é finito. Assim, $(B \cup g(B)) \Delta(A \cup f(A))$ é finito. Devemos ter então que $A \backslash B$ e $B \backslash A$ são finitos. Mas como $A \cup B=(A \Delta B) \cup(A \cap B)$ e $A \cap B$ é finito quando $A \neq B$, segue que $A=B$. Assim, temos que $(A \cup g(A)) \Delta(A \cup f(A))$ é finito, e segue que $g(A) \Delta f(A)$ é finito. Como antes, segue que $g(A)=f(A)$ e $\phi(A \cup f(A))=A \cup f(A)$. Como $A$ é arbitrário, segue a tese.

d) Temos que

$\left\{f \in F: \Psi(\mathcal{C}) \approx \psi\left(\mathcal{A}_{f}\right)\right\}=\bigcup_{h \in \omega_{\omega}}\left\{f \in F: \exists \phi: \Psi(\mathcal{C}) \rightarrow \Psi\left(\mathcal{A}_{f}\right)\right.$ homeomorfismo tal que $\left.\phi \mid \omega=h\right\}$

Assim, basta mostrar que para cada $h: \omega \rightarrow \omega$ bijetora, temos que $\{f \in F: \exists \phi: \Psi(\mathcal{C}) \rightarrow$ $\Psi\left(\mathcal{A}_{f}\right)$ homeomorfismo tal que $\left.\phi \mid \omega=h\right\}$ é finito. Na verdade, mostraremos que esse conjunto tem no máximo um elemento. Suponha que $\phi_{f}, \phi_{g}$ são homeomorfismos, respectivamente, de $\Psi(\mathcal{C})$ em $\Psi\left(\mathcal{A}_{f}\right)$ e de $\Psi(\mathcal{C})$ em $\Psi\left(\mathcal{A}_{g}\right) \operatorname{com} \phi_{f}\left|\omega=\phi_{g}\right| \omega=h$. Então $\phi_{g} \circ \phi_{f}^{-1}: \Psi\left(\mathcal{A}_{f}\right) \rightarrow \Psi\left(\mathcal{A}_{g}\right)$ é um homeomorfismo que fixa $\omega$. Logo, $f=g$ e $\phi_{f}=\phi_{g}$.

Corolário 2.32. Existem $2^{\mathfrak{c}}$ MAD families com $\Psi$-espaços não dois-a-dois homeomorfos. Assim, existem ao menos $2^{\mathfrak{c}}$ espaços pseudocompactos, Hausdorff, zero dimensionais, separáveis e localmente compactos não dois-a-dois homeomorfos. 
Demonstração. Na proposição anterior, tomemos $\kappa=\mathfrak{c}$ (existem MAD families de cardinalidade $\mathfrak{c}$, então isso é possível). Seja $\mathcal{W}=\left\{A_{f}: f \in F\right\}$. Pelo item d) da proposição anterior, a relação de equivalência em $W$ dada pela relação de ser homeomorfo particiona $\mathcal{W}$ em classes de no máximo $\mathfrak{c}$ elementos. Porém, pelo item b), $|\mathcal{W}|=2^{\mathfrak{c}}$. Logo, existem $2^{\mathfrak{c}}$ dessas classes de equivalência e segue a tese.

\subsection{Um pouco sobre $\beta \omega$}

Esta seção estava originalmente pensada para ser parte do capítulo introdutório. Porém, ao fim da seção, faremos uma aplicação de almost disjoint families. Assim, decidimos por movê-la para este capítulo. Utilizaremos o espaço $\beta \omega$ em algumas construções nas demais seções.

O peso de um espaço topológico $X$ é definido como $\mathrm{w}(X)=\min \{|\mathcal{B}|: \mathcal{B}$ é base de $X\}+\omega$.

Lema 2.33. Seja $\kappa$ um cardinal infinito. Então $\mathrm{w}\left({ }^{\kappa} 2\right)=\kappa$.

Demonstração. Temos que $\left\{V_{s}: s \in \bigcup_{F \in[\kappa]<\omega} 2^{f}\right\}$, onde $V_{s}=\left\{f \in 2^{\kappa}: s \subseteq f\right\}$ é uma base de $2^{\kappa}$ de cardinalidade $\kappa$. Basta mostrar que não existem bases de cardinalidade menor. Temos que $\mathcal{B}=\left\{\left\{f \in{ }^{\kappa} 2: f(\alpha)=i\right\}: i \in\{0,1\}, \alpha<\kappa\right\}$ é uma subbase de ${ }^{\kappa} 2$ de cardinalidade $\kappa$.

Denotemos por $U(\alpha, i)$ o conjunto $\left\{f \in{ }^{\kappa} 2: f(\alpha)=i\right\}$ Se $\mathcal{B}$ é uma coleção de $\lambda<\kappa$ abertos básicos, podemos escrever $\mathcal{B}=\left\{V_{\beta}: \beta<\lambda\right\}$ onde

$$
V_{\beta}=\bigcap_{i<n_{\beta}} U\left(\alpha_{0}^{\beta}, j_{i}^{\beta}\right)
$$

Para algum $n_{\beta}>0$ natural, alguma $\left(\alpha_{i}^{\beta}\right)_{i<n_{\beta}}$ sequência de ordinais menores do que $\lambda$ e $\left(j_{i}^{\beta}\right)_{i<n_{\beta}}$ sequência em $\{0,1\}$. Temos que o conjunto $\left\{\alpha_{i}^{\beta}: \beta<\lambda, i<n_{\beta}\right\}$ tem cardinalidade $\leq \lambda<\kappa$, então existe $\gamma$ fora dele. Temos que não existe $\beta$ tal que $V_{\beta} \subseteq U(\alpha, 0)$, logo, $\mathcal{B}$ não é base.

Proposição 2.34. Suponha que $\kappa$ seja um cardinal infinito, que $|I| \leq 2^{\kappa}$ e que para todo $\alpha \in I$, $X_{\alpha}$ é um espaço topológico e $D_{\alpha} \subseteq X_{\alpha}$ é um denso tal que $\left|D_{\alpha}\right| \leq \kappa$. Então $\prod_{\alpha \in I} X_{\alpha}$ possui um denso de cardinalidade $\leq \kappa$.

Demonstração. Sem perda de generalidade, $I={ }^{\kappa} 2$. Seja $\mathcal{B}$ uma base de ${ }^{\kappa} 2$ de cardinalidade $\kappa$. Para cada $f \in I$, seja $D_{f}=\left\{d_{\beta}^{f}: \beta \leq \kappa\right\}$ (repetindo se necessário). Para cada número natural $k$, para cada sequência $\left(A_{1}, \ldots, A_{k}\right)$ de elementos de elementos disjuntos de $\mathcal{B}$, e $\left(\beta_{1}, \ldots, \beta_{1}\right)$ sequência em $\kappa+1$, seja $p=p\left(\left(A_{1}, \ldots, A_{k}\right),\left(\beta_{1}, \ldots, \beta_{k}\right)\right)$ dado por:

$$
\begin{gathered}
p(f)=d_{\beta_{i}}^{f}, \text { se } f \in A_{i} \\
p(f)=d_{0}^{f}, \text { caso contrário. }
\end{gathered}
$$

O conjunto de todas as funções definidas como acima tem cardinalidade $\leq \kappa$. Resta ver que ele é denso. Consideremos um aberto básico do produto. Ele tem a forma

$$
\pi_{f_{1}}^{-1}\left(U_{1}\right) \cap \cdots \cap \pi_{f_{m}}^{-1}\left(U_{m}\right)
$$

Onde $m \in \omega$ é não nulo, $f_{i}: \kappa \rightarrow 2, U_{i} \subseteq X_{f_{i}}$ é aberto não vazio. Cada $U_{i}$ possui um elemento da forma $d_{\beta_{i}}^{f_{i}}$. Como ${ }^{\kappa} 2$ é Hausdorff, existem $A_{1}, \ldots, A_{m} \in \mathcal{B}$ disjuntos com $f_{i} \in A_{i}$. Assim, temos que para cada $1 \leq i \leq m$ :

$$
p\left(\left(A_{1}, \ldots, A_{m}\right),\left(\beta_{1}, \ldots, \beta_{m}\right)\right)\left(f_{i}\right)=d_{\beta_{i}}^{f_{i}} \in U_{i}
$$

Assim, $p\left(\left(A_{1}, \ldots, A_{m}\right),\left(\beta_{1}, \ldots, \beta_{m}\right)\right) \in \pi_{f_{1}}^{-1}\left(U_{1}\right) \cap \cdots \cap \pi_{f_{m}}^{-1}\left(U_{m}\right)$ e segue a tese.

Proposição 2.35. Se $X$ é infinito e discreto, então $|\beta X|=2^{2^{|X|}}$. Em particular, $|\beta \omega|=2^{\mathfrak{c}}$. 
Demonstração. Seja $\kappa=|X|$. Temos pela proposição anterior que $[0,1]^{2^{\kappa}}$ possui um subconjunto $D$ denso de cardinalidade $\lambda \leq \kappa$. Seja $f: X \rightarrow D$ sobrejetora. Seja $\beta f: X \rightarrow D$ a extensão de $f$ à $[0,1]^{2^{\kappa}}$. Como $\beta X$ é compacto e $D$ é denso, temos que $\beta f[\beta X]=[0,1]^{2^{\kappa}}$. Logo:

$$
|\beta X| \geq\left|[0,1]^{2^{\kappa}}\right|=2^{\omega 2^{\kappa}}=2^{\omega 2^{\kappa}}=2^{2^{\kappa}}
$$

Por outro lado, vimos que $\beta X$ é o conjunto de todos os ultrafiltros em $X, \operatorname{logo}, \beta X \subseteq \mathscr{P}(\mathscr{P}(X))$, assim, $|\beta X| \leq 2^{2^{\kappa}}$

Corolário 2.36. Todo conjunto infinito $X$ possui exatamente $2^{2^{|X|}}$ ultrafiltros livres e $|X|$ ultrafiltros fixos. Em particular, $|\beta \omega|=\left|\omega^{*}\right|=2^{\mathfrak{c}}$.

Antes de prosseguir, provaremos o seguinte fato elementar sobre espaços de Hausdorff:

Proposição 2.37. Suponha que $X$ é um espaço de Hausdorff infinito. Então $X$ possui um subconjunto infinito e discreto.

Demonstração. Primeiro, mostraremos que se $X$ é Hausdorff e infinito, existe $U$ aberto tal que $X \backslash \operatorname{cl} U$ é infinito. Se isso não for possível, tomemos $U$ aberto não tal que $\operatorname{cl} U \neq X$, o que é possível fixando dois pontos distintos de $X$ e separando eles por abertos. Então $X \backslash \operatorname{cl} U$ é finito e Hausdorff, logo, discreto. Assim, dado $x \in X \backslash \operatorname{cl} U$, temos que $\{x\}$ é clopen. Logo, $V=\{x\}$ é aberto e $X \backslash \mathrm{cl} V=X \backslash\{x\}$ é infinito, absurdo.

Agora, definimos recursivamente uma sequência infinita de abertos dois-a-dois disjuntos $U_{n}$ da seguinte forma: tomamos $U_{0}$ aberto não vazio tal que $X \backslash \operatorname{cl} U_{0}$ é infinito. Definido $U_{n}(n \leq m)$ abertos não vazios disjuntos dois-a-dois de modo que $X \backslash \bigcup_{n \leq m} \operatorname{cl} U_{n}$ seja infinito, define-se $U_{m+1}$ como um aberto não do espaço Hausdorff $Y=X \backslash \bigcup_{n \leq m} \operatorname{cl} U_{n}$ de modo que $Y \backslash \operatorname{cl} U_{m+1}$ seja infinito. Agora, tomando $x_{n} \in U_{n}$ para todo $n$, temos que o espaço $\left\{x_{n}: n \in \omega\right\}$ é infinito e discreto.

Proposição 2.38. Seja $F \subseteq \beta \omega$ infinito e fechado. Então $|F|=2^{\mathfrak{c}}$.

Demonstração. Nessa demonstração, escreveremos $\omega$ como sendo o conjunto dos ultrafiltros fixos (ou seja, identificaremos $\omega$ dentro de $\beta \omega$ ).

Temos que como $F$ é Hausdorff, $F$ contém um subespaço infinito enumerável e discreto, que chamaremos de $E$. Temos que $\omega \cup E$ é regular e enumerável, logo, normal. Temos que $\omega$ é aberto em $\omega \cup E$, logo $E$ é fechado em $\omega \cup E$. Veremos que $\operatorname{cl}_{\beta \omega} E \approx \beta E$. Como $\operatorname{cl}_{\beta \omega} E \subseteq E$ e $|\beta E|=2^{\mathfrak{c}}$, seguirá a tese. Como cl $E$ é uma compactificação de $E$, basta ver que $E$ é $C^{*}$-imerso em cl $E$. Dada $f: E \rightarrow[a, b]$ contínua, pelo Teorema de Tietze existe $g: \omega \cup E \rightarrow[a, b]$ contínua tal que $f \subseteq g$. Seja $h=g \mid \omega$. Temos que $h: \omega \rightarrow[a, b]$ é contínua. Tomemos a extensão contínua $\beta h: \beta \omega \rightarrow[a, b]$ e consideremos $p=\beta h \mid \operatorname{cl} E: \operatorname{cl} E \rightarrow[a, b]$, que é contínua. Precisamos ver que $f \subseteq p$. Basta ver que $f \subseteq \beta h$, e, para isso, basta ver que $g \subseteq \beta$. Para isso, basta ver que $g=(\beta h \mid E \cup \omega)$. Temos que ambas essas funções coincidem em $\omega$ (a restrição de ambas é h) e $\omega$ é denso em $\omega \cup E$, logo, as funções coincidem em $E \cup \omega$ e segue a tese.

Dessa demonstração, podemos retirar o seguinte corolário:

Corolário 2.39. Todo subconjunto enumerável de $\beta \omega$ é $C^{*}$-imerso.

Corolário 2.40. Todo subconjunto $A$ aberto de $\beta \omega$ que intersecta $\omega^{*}$ tem cardinalidade $2^{\mathfrak{c}}$. Em particular, todo subconjunto aberto e infinito de $\omega^{*}$ tem cardinalidade $2^{\mathfrak{c}}$, e todo subconjunto aberto não enumerável de $\beta \omega$ tem cardinalidade $2^{\mathfrak{c}}$.

Demonstração. Seja $x \in \omega^{*} \cap A$. Pela regularidade de $\beta \omega$, existe $U$ aberto com $x \in U \subseteq \operatorname{cl} U \subseteq A$. Precisamos ver que $\operatorname{cl} U$ é infinito. Se for finito, temos que $x \in \operatorname{cl} U=\operatorname{cl}(\omega \cap U)=\omega \cap U$, logo $x \in \omega$, absurdo.

Corolário 2.41. $\omega^{*}$ não tem pontos isolados. 
Definição 2.10. Seja $D$ infinito e discreto. Se $A \subseteq D$, denota-se por $A^{*} \subseteq \beta D$ a coleção dos $p$ ultrafiltros livres em $D$ tais que $A \in p$.

Lema 2.42. Seja $D$ infinito e discreto. Então, no espaço $D^{*}$ :

1. Para todo $A \subseteq D,\{p \in \beta D: A \in D\}$ é um clopen de $\beta D$.

2. $\{\{p \in \beta D: A \in D\}: A \subseteq D\}$ é uma base de $\beta D$.

3. $\left\{A^{*}: A \subseteq D\right\}$ é uma base de clopens de $D^{*}$.

4. Se $A, B \subseteq D$, então $A \subseteq{ }^{*} B$ se, e somente se, $A^{*} \subseteq B^{*}$.

5. Se $A, B \subseteq D$, então $(A \cap B)^{*}=A^{*} \cap B^{*}$.

6. Se $A, B \in D$, temos que $A \cap B$ é finito se, e somente se, $A^{*} \cap B^{*}=\emptyset$

Demonstração. Seja $D$ infinito e discreto.

1. Se $A \subseteq D$, temos que $\{p \in \beta D: A \in p\}=D \backslash\{p \in \beta D: A \notin p\}$ é um fechado de $\beta D$. Porém, seu complemento $\{p \in \beta D: X \backslash A \in p\}$ também é um fechado de $\beta D$. Logo, $\{p \in \beta D: A \in p\}$ é fechado.

2. Já vimos que dado $A \subseteq D,\{p \in \beta D: A \in D\}$ é aberto. Temos que $\beta D=\{p \in \beta D: A \in X\}$. Finalmente, dado $p \in \beta D$ e $A$ aberto, temos que existe $A \subseteq D$ tal que $p \in\{p \in \beta D: A \notin$ $D\} \subseteq A$. Porém, $\{p \in \beta D:(X \backslash A) \in D\}=\{p \in \beta D: A \notin D\}$, assim, segue a tese.

3. Como para todo $A \subseteq D$ temos que $A^{*}=D^{*} \cap\{p \in \beta D: A \in D\}$, este item segue diretamente dos dois anteriores.

4. Suponha que $A \subseteq \subseteq^{*} B$. Sendo $p$ um ultrafiltro livre tal que $A \in p$, existe $C$ cofinito tal que $A \cap C \subseteq B$. Como $C \in p$, temos que $B \in p$. Assim, $A^{*} \subseteq B^{*}$. Reciprocamente, suponha que $A \backslash B$ é infinito. Seja $p$ ultrafiltro livre tal que $A \backslash B \in p$. Segue que $p \in A^{*} \backslash B^{*}$.

5. Seja $p$ um ultrafiltro livre. Temos que $A \cap B \in p$ se, e somente se, $A, B \in p$.

6. Temos que $A \cap B$ é finito se, e somente se, $A \cap B \subseteq^{*} \emptyset$ se, e somente se, $(A \cap B)^{*} \subseteq \emptyset^{*}$ se, e somente se, $A^{*} \cap B^{*} \subseteq \emptyset$ se, e somente se, $A^{*} \cap B^{*}=\emptyset$.

A proposição abaixo é outra aplicação interessante de almost disjoint families.

Proposição 2.43. A celularidade e o peso de $\omega^{*}$ são $\mathfrak{c}$.

Demonstração. Pelo lema anterior, $\left\{A^{*}: A \subseteq D\right\}$ é uma base de clopens de $\omega^{*}$. Assim, precisamos apenas ver que a celularidade de $\omega^{*}$ é pelo menos $\mathfrak{c}$. Seja $\mathcal{A}$ uma almost disjoint family de cardinalidade $\mathfrak{c}$. Pelo lema anterior, $\left\{A^{*}: A \in \mathcal{A}\right\}$ é uma coleção de $\mathfrak{c}$ abertos dois à dois disjuntos. Assim, segue a tese.

Proposição 2.44. Se $F \subseteq \omega^{*}$ é tal que $|F|<\mathfrak{c}$, então $F$ é nowhere dense, ou seja, o interior do fecho de $F$ é vazio.

Demonstração. Suponha por absurdo que o int cl $F \neq \emptyset$. Então existe $A \subseteq \omega$ infinito tal que $A^{*} \subseteq \operatorname{cl} F$. Seja $\left\{A_{\alpha}: \alpha<\mathfrak{c}\right\}$ uma almost disjoint family em $A$ tal que se $\alpha \neq \beta$ então $A_{\alpha} \cap A_{\beta}$ é finito. Temos que para todo $\alpha, A_{\alpha}^{*} \subseteq \operatorname{cl} F$, e, como se $\alpha \neq \beta$ então $A_{\alpha} \cap A_{\beta}$ é finito, segue que $A_{\alpha}^{*} \cap A_{\beta}=\emptyset$. Como cada $A_{\alpha}$ é infinito, temos que $A_{\alpha}^{*} \neq \emptyset$. Assim, como $A_{\alpha}^{*} \subseteq \operatorname{cl} F$, temos que $A_{\alpha}^{*} \cap F \neq \emptyset$. Para cada $\alpha$, tome $p_{\alpha} \in A_{\alpha}^{*} \cap F$. Segue que $|F| \geq \mathfrak{c}$, o que é absurdo. 


\subsection{Almost distoint families e happy families}

Nessa seção, seguimos as definições do artigo [Mat77]. Mostraremos que $\mathcal{I}^{+}(\mathcal{A})$ é uma happy family para toda MAD family $\mathcal{A}$.

Definição 2.11. $X \subseteq \omega$ diagonaliza a família $\left(X_{s}: s \in[\omega]<\omega\right)$ de subconjuntos de números naturais se, e somente se, for infinito, $X \subseteq X_{\emptyset}$ e para todo $s \in[\omega]^{<\omega}$, se $\max s \in X$ então $X \backslash\left(\bigcup_{n \in s} n+1\right) \subseteq X_{s}$. Para abreviar essa sentença, define-se $o(s)=\left(\bigcup_{n \in s} n+1\right)$. Note que $o(s)=0$ quando $s=\emptyset$, e $o(s)=\max s+1$ caso contrário.

Definição 2.12. $A \subseteq \mathscr{P}(\omega)$ é dita uma happy family se $\mathscr{P}(\omega) \backslash A$ é um ideal livre e para toda família de conjuntos de números naturais $\left(X_{s}: s \in[\omega]^{<\omega}\right)$, se $\left\langle\left\{X_{s}: s \in[\omega]^{<\omega}\right\} \cup \operatorname{Fr}\right\rangle \subseteq A$ (o filtro livre gerado por $\left(X_{s}: s \in[\omega]<\omega\right)$ ), existe $X \in A$ que diagonaliza $\left(X_{s}: s \in[\omega]<\omega\right)$.

Como um exemplo, segue o lema abaixo.

Lema 2.45. Suponha que $\left(X_{s}: s \in[\omega]^{<\omega}\right)$ é uma família de subconjuntos infinitos de $\omega$ com a propriedade da interseção finita forte. Então existe $X$ que diagonaliza $\left(X_{s}: s \in[\omega]^{<\omega}\right)$.

Demonstração. Fixe $n_{0} \in X_{\emptyset}$. Definido $n_{k}$, tomemos $n_{k+1} \in \bigcap\left\{X_{s}: o(s) \leq n_{k}+1\right\} \operatorname{com} n_{k+1}>n_{k}$, o que é possível já que essa interseção é infinita. Seja $X=\left\{n_{k}: k<\omega\right\}$. Então $X \subseteq X_{\emptyset}$. Suponha que $s \subseteq \omega$ é finito com $\max s=n_{k} \in X$. Temos que $X \backslash\left(n_{k}+1\right)=\left\{n_{l}: l \geq k+1\right\}$. Notemos que se $l \geq k+1$, então $n_{l} \in X_{s}$, assim, segue a tese.

Proposição 2.46. Seja $\mathcal{A}$ uma almost disjoint family. Então $\mathcal{I}(\mathcal{A})$ é um ideal livre próprio, e $\mathcal{I}(\mathcal{A})$ é um ideal primo e alto se e somente se $\mathcal{A}$ é uma MAD family.

Demonstração. Primeiro, verifiquemos que $\mathcal{I}(\mathcal{A})$ é um ideal livre. Claramente, todo subconjunto finito de $\omega$ é elemento de $\mathcal{I}$, que se $A, B \in \mathcal{I}(\mathcal{A})$ então $A \cup B \in \mathcal{I}(\mathcal{A})$ e que se $A \in \mathcal{I}(\mathcal{A})$ e $B \subseteq A$ então $B \in \mathcal{I}(\mathcal{A})$. Verificaremos que $\omega \notin \mathcal{I}(\mathcal{A})$. Se isso ocorrer, existem $B_{1}, \ldots, B_{k} \in \mathcal{A}$ com $\omega \subseteq \subseteq^{*} B_{1} \cup \cdots \cup B_{k}$. Isso significa que existe $n$ natural com $B_{1} \cup \cdots \cup B_{k} \cup n=\omega$. Tomemos $A \in \mathcal{A}$ distinto de $B_{1}, \ldots, B_{k}$. Temos que $A=\left(B_{1} \cap A\right) \cup \cdots \cup\left(B_{k} \cap A\right) \cup(n \cap A)$. Mas o lado direito é uma união finita de conjuntos finitos, logo, $A$ é finito, absurdo.

Agora suponha que $\mathcal{A}$ é MAD. Para ver que nosso ideal é alto, basta observar que dado $X \subseteq \omega$ infinito, existe, pela maximalidade de $\mathcal{A}$, um $B \in \mathcal{A}$ tal que $B \cap X$ é infinito.

Reciprocamente, suponhamos que $\mathcal{I}(\mathcal{A})$ é um ideal alto. Veremos que $\mathcal{A}$ é maximal. Suponha que $B \subseteq \omega$ é infinito. Existe $X \in \mathcal{I}(\mathcal{A})$ tal que $X \cap B$ é infinito. Existem $A_{1}, \ldots, A_{k} \in \mathcal{A}$ e $n \in \omega$ tais que $X \subseteq A_{1} \cup \cdots \cup A_{k} \cup n$. Logo, como $B \cap\left(A_{1} \cup \cdots \cup A_{k} \cup n\right)$ é infinito, existe $i$ tal que $B \cap A_{i}$ é infinito e segue a tese.

É interessante notar que o fato de $\mathcal{A}$ ser infinito foi importante na proposição acima.

Proposição 2.47. Seja $\mathcal{A}$ uma MAD family. Então $\mathcal{I}^{+}(\mathcal{A})$ é uma happy family.

Demonstração. Tomemos uma família de subconjuntos de números naturais $\left(X_{s}: s \in[\omega]<\omega\right)$ tal que $\left\langle\left\{X_{s}: s \in[\omega]^{<\omega}\right\} \cup \operatorname{Fr}\right\rangle \subseteq \mathcal{A}$.

Construiremos recursivamente sequências $X^{n}, Y^{n}$ e $X_{s}^{n}$ para cada $n \in \omega, s \in[\omega]^{<\omega}$ com as seguintes propriedades:

(a) $X_{s}^{0}=X_{s}$,

(b) cada $X_{s}^{n}$ é infinito,

(c) $X^{n}$ diagonaliza $\left(X_{s}^{n}: s \in[\omega]^{<\omega}\right)$,

(d) $Y^{n} \in \mathcal{A}$ e $Y^{n} \cap X^{n}$ é infinito,

(e) $X_{s}^{n+1}=X_{s}^{n} \backslash\left(\bigcup_{i \leq n} Y^{i}\right)$, 
(f) $\left\langle\left\{X_{s}^{n}: s \in[\omega]^{<\omega}\right\} \cup \operatorname{Fr}\right\rangle \subseteq \mathcal{I}^{*}(\mathcal{A})$.

Começamos definindo $X_{s}^{0}=X_{s}$, como em $(a)$. Pelo lema anterior, existe $X^{0}$ que diagonaliza $\left(X_{s}^{0}: s \in[\omega]^{<\omega}\right)$. Pela maximalidade de $\mathcal{A}$, existe $Y^{0} \in \mathcal{A}$ tal que $Y^{n} \cap X^{0}$ é infinito. Notemos que (f) é decorre de (a) para $n=0$.

Definidas as sequências até $n$, define-se $X_{s}^{n+1}$ como em $(d)$. (b) É satisfeito para $n+1$ pois para cada $s, X_{s}^{n} \in I^{*}(\mathcal{A})$, e (f) é satisfeito pois $X_{s}^{n+1} \subseteq X_{s}^{n}$ para cada $s$. Como vale (f) para $n+1$, pelo lema anterior existe $X^{n+1}$ infinito que diagonaliza $\left(X_{s}^{n+1}: s \in[\omega]^{<\omega}\right)$, e, pela maximalidade de $\mathcal{A}$, existe $Y^{n+1}$ que intersectado com $X^{n+1}$ é infinito.

Temos que $X_{s}^{j}=X_{s}^{i} \backslash\left(\bigcup_{k<j} Y^{k}\right)$ para todo $s$.

Notemos que se $i<j$ então $Y^{i} \neq Y^{j}$ : Temos que $X^{j} \subseteq X_{0}^{j}=X_{0}^{i} \backslash\left(\bigcup_{k<j} Y^{k}\right)$. Temos que existe $x$ em $X^{j} \cap Y^{j}$, logo, esse $x$ não está em $Y^{i}$.

Como para cada $n, s$, temos que $X_{s}^{n} \subseteq X_{s}$, e como $X^{n}$ diagonaliza $\left(X_{s}^{n}: s \in[\omega]^{<\omega}\right)$, temos que $X^{n}$ diagonaliza $\left(X_{s}: s \in[\omega]^{<\omega}\right)$.

Agora recursivamente, construiremos uma sequência estritamente crescente de números naturais $n_{k}$ tal que $n_{0} \in X_{\emptyset}$ e, se $k=2^{j}(2 l+1)$, então $n_{k} \in Y^{j} \cap X^{j} \backslash\left(n_{k}+1\right)$.

Seja $Z=\left\{n_{k}: j<\omega\right\}$. Temos que $Z$ é infinito. Para cada $j, Z \cap Y^{j}$ é infinito pois o conjunto $\left\{n_{2^{j}(2 l+1)}: l \in \omega\right\}$ está contido na interseção. Segue que $Z \in \mathcal{I}^{*}(\mathcal{A})$ é infinito: do contrário existiriam $U_{1}, \ldots, U_{k} \in \mathcal{A}$ e um natural $u$ tais que $Z \subseteq U_{1} \cup \cdots \cup U_{k} \cup u$. Tomando $Y_{i}$ distinto de cada $U_{j}$, temos que $Z \cap Y_{i}$ é infinito mas $\left(U_{1} \cup \cdots \cup U_{k} \cup u\right) \cap Y_{i}$ é finito, absurdo. Resta ver que $Z$ diagonaliza nossa família. Claramente, $Z$ é infinito.

Dado $k, n_{k} \in X^{l} \subseteq X_{\emptyset}$, para algum $l$. Assim, $Z \subseteq X^{0}$. Dado $s \neq 0$, suponha que $n_{k}=\max s \in Z$. Devemos ver que $Z \backslash\left(n_{k}+1\right) \subseteq X_{s}$. Temos que $Z \backslash\left(n_{k}+1\right)=\left\{n_{l}: l \geq k+1\right\}$. Fixado $l$ com $l \geq k+1$, temos que existe $j$ tal que $n_{l} \in Y^{j} \cap X^{j} \backslash\left(n_{k}+1\right)$. Como $X^{j}$ diagonaliza a nossa família, temos que $n_{l} \in X^{j} \backslash n_{k} \subseteq X_{s}$.

Abaixo, veremos uma equivalência útil sobre happy families que nos trarão uma propriedade útil acerca de MAD families.

Teorema 2.48. Suponha que $A \subseteq \mathscr{P}(\omega)$ seja tal que $\mathscr{P}(\omega) \backslash A$ seja um ideal livre. Então $A$ é uma happy family se, e somente se, para toda sequência decrescente $\left(Y_{i}: i \in \omega\right)$ de elementos de $A$ existe $f: \omega \rightarrow \omega$ estritamente crescente tal que $f[\omega] \in A$ e para todo $n, f(n+1) \in Y_{f(n)}$.

Demonstração. Suponha que $A$ seja uma happy family e considere uma sequência $Y_{i}(i \in \omega)$ decrescente de elementos de $A$. Para $s \in[\omega]^{\omega}$, seja $X_{s}=Y_{o(s)-1}$, se $s \neq 0$, e $X_{\emptyset}=Y_{0}$. Devemos ver que $\left\langle\left\{Y_{i}: i<\omega\right\} \cup \operatorname{Fr}\right\rangle \subseteq A$. Suponha que $X$ esteja nesse conjunto. Existe $i<A$ e $B$ cofinito tal que $Y_{i} \cap B \subseteq X$. Logo, $\omega \backslash X \subseteq \omega \backslash\left(Y_{i} \cap B\right)=\left(\omega \backslash Y_{i}\right) \cup(\omega \backslash B)$. Como $C=\mathscr{P}(\omega) \backslash A$ é um ideal primo, temos que $\omega \backslash Y_{i} \in C$ (pois seu complementar não está em $C$ ) e $\omega \backslash B \in C$ (pois este é finito). Logo, sua união está em $C$. Assim, seu complemento $Y_{i} \cap B$ não está em $C$, logo, está em $A$.

Tome $X \in A$ que diagonalize $\left(X_{s}: s \in[\omega]^{<\omega}\right)$ e $f: \omega \rightarrow X$ enumeração em ordem crescente. Dado $n$, seja $s=X \cap(f(n)+1)$. Como $f(n)=\max s$, temos que $o(s)=f(n)+1$, e como $s \subseteq X$, temos que $\sup s \in X$. Temos que $f(n+1) \subseteq X \backslash o(s) \subseteq X_{s}=Y_{f(n)}$, como queríamos.

Reciprocamente, suponha que valha essa propriedade para $A$, e suponha que o filtro gerado por $\left(X_{s}: s \in[\omega]^{<\omega}\right) \cup$ Fr está contido em $A$.

Para cada $i$, seja $Y_{i}=\bigcap\left\{X_{s}: o(s) \leq i+1\right\}$. Então $Y_{i}$ é uma sequência decrescente de subconjuntos de $A$. Tome $f$ como no enunciado e seja $X=f[\omega] \backslash\{f(0)\}$. Temos que $X \in A$ pois $\omega \backslash(X \backslash\{f(0)\})=(\omega \backslash X) \cup\{f(0)\} \in \mathscr{P}(\omega) \backslash A, \operatorname{logo}, X \backslash\{f(0)\} \notin \mathscr{P}(\omega) \backslash A$. Temos que $X \subseteq Y_{0} \subseteq X_{\emptyset}$. Se $s \in[\omega]^{<\omega}$ é tal que $\max s \in X$, existe $n$ tal que $f(n)=\max s$. Temos que $o(s)=f(n)+1$. Se $k \in X \backslash o(s)$, temos que existe $m>n$ tal que $k=f(m)$. Daí, $k \in Y_{f(m)-1} \subseteq Y_{f(n)} \subseteq X_{s}$. Assim, segue a tese. 
Corolário 2.49. Seja $\mathcal{A}$ uma MAD family e $X_{n}$ uma sequência decrescente de $I^{+}(\mathcal{A})$. Então existe $X$ em $I^{+}(\mathcal{A})$ tal que para todo $n \in \omega, X \subseteq^{*} X_{n}$.

Demonstração. Na notação do enunciado, $I^{+}(\mathcal{A})$ é uma happy family. Existe $f: \omega \rightarrow \omega$ estritamente crescente tal que $f[\omega] \in I^{*}(\mathcal{A})$ e para todo $n, f(n+1) \in X_{f(n)}$. Seja $X=f[\omega]$. Dado $n$, existe $m$ tal que $f(m)>n$. Temos que $X \backslash f(m+1) \subseteq X_{f(m)} \subseteq X_{n}$.

Como corolário, podemos ver que a propriedade acima vale para qualquer almost disjoint family.

Corolário 2.50. Seja $\mathcal{A}$ uma almost disjoint family e $X_{n}$ uma sequência decrescente em $I^{+}(\mathcal{A})$. Então existe $X$ em $I^{+}(\mathcal{A})$ tal que para todo $n \in \omega, X \subseteq^{*} X_{n}$.

Demonstração. Seja $U=\left\{\mathcal{M} \in \mathscr{P}(\omega): \mathcal{A} \subseteq M\right.$ é adf e $\left.\forall n\left(X_{n} \in I^{+}(\mathcal{M})\right)\right\}$. Pelo Lema de Zorn, existe $\mathcal{M}$ maximal em $U$. Veremos que $\mathcal{M}$ é MAD family. Suponha que não. Então vale:

$$
\exists Y \forall B \in \mathcal{M}(|Y \cap B|<\omega)
$$

Dado $Y$ dessa forma, pela maximalidade de $\mathcal{M}$, existe $n$ tal que $X_{n} \in \mathcal{I}(\mathcal{M} \cup\{Y\})$. Assim, vale que:

$$
\exists n \exists Y \forall B \in \mathcal{M}\left(|Y \cap B|<\omega \text { e } X_{n} \in \mathcal{I}(\mathcal{M} \cup\{Y\})\right)
$$

Procederemos por indução. Para fins de melhor compreensão, faremos os dois primeiros passos da indução antes de fazer o passo indutivo. Seja $n_{0}$ o menor $n$ que satisfaz a fórmula acima e fixe $Y_{0}$ que ateste isso.

Agora, recursivamente, escolhemos uma sequência de números naturais estritamente crescente $n_{k}$ e uma sequência decrescente de subconjuntos infinitos de $\omega, Y_{k}$, que satisfaz:

(a) $\left|Y_{k} \cap B\right|<\omega$ para todo $B \in \mathcal{M}$

(b) $X_{n_{k}} \in \mathcal{I}\left(\mathcal{M} \cup\left\{Y_{k}\right\}\right)$,

(c) Se $n \leq n_{k}, X_{n} \in \mathcal{I}^{*}\left(\mathcal{M} \cup\left\{Y_{k+1}\right\}\right)$,

Escolhemos $n_{0}, Y_{0}$ como no parágrafo precedente. Escolhidos $n_{0}, \ldots, n_{k}$ e $Y_{0}, \ldots, Y_{k}$, por (b), Existem $m$ natural e $B_{1}, \ldots, B_{k} \in \mathcal{M}$ tais que $X_{n_{k}} \subseteq m \cup B_{1} \cup \ldots B_{k} \cup Y_{k}$. Temos que $X_{n_{k}} \backslash Y_{k} \subseteq$ $m \cup B_{1} \cup \cdots \cup B_{k}$, logo, $X_{n_{k}} \backslash Y_{k} \in \mathcal{I}(\mathcal{M})$. Logo, temos que $X_{n_{k}} \cap Y_{k} \notin \mathcal{I}(\mathcal{M})$, ou teríamos que $X_{n_{k}} \in \mathcal{I}(\mathcal{M})$. Sejam $Y_{k+1}, Y_{k+1}^{\prime}$ infinitos e disjuntos tais que $X_{n_{k}} \cap Y_{k}=Y_{k+1} \cup Y_{k+1}^{\prime}$. Um destes não pertence à $\mathcal{I}(\mathcal{M})$, sem perda de generalidade podemos supor que é $Y_{k+1}^{\prime}$. Temos que $X_{n_{k}} \notin \mathcal{I}(\mathcal{M} \cup$ $\left\{Y_{k+1}\right\}$ ), ou teríamos que $X_{n_{k}} \backslash Y_{k+1} \in \mathcal{I}(\mathcal{M})$, o que implica em $Y_{k+1}^{\prime}=X_{n_{k}} \cap Y_{k} \backslash Y_{k+1} \in \mathcal{I}(\mathcal{M})$. Dessa forma, se $n<n_{k}$, também vale que $X_{n} \notin \mathcal{I}\left(\mathcal{M} \cup\left\{Y_{k+1}\right\}\right)$. Pela maximalidade de $\mathcal{M}$, existe $n_{k+1}>n_{k} \operatorname{com} X_{n_{k+1}} \in \mathcal{I}\left(\mathcal{M} \cup\left\{Y_{k+1}\right\}\right)$.

Agora seja $Y$ uma pseudointerseção de todos os $Y_{k}$. Como $Y \backslash Y_{0}$ é finito, segue que $|Y \cap B|<\omega$ para todo $B \in \mathcal{M}$. Dado $n$, temos que $X_{n} \in \mathcal{I}^{*}(\mathcal{M} \cup\{Y\})$ : fixe $k$ tal que $n_{k}>n$ e suponha por absurdo que $X_{n} \subseteq B_{1} \cup \ldots B_{k} \cup m \cup Y$ para algum $m$ natural e $B_{1} \ldots, B_{k} \in \mathcal{M}$. Seja $l>m$ natural tal que $Y \backslash Y_{k} \subseteq l$. Temos que $X_{n} \subseteq B_{1} \cup \ldots B_{k} \cup l \cup Y_{k}$. Absurdo. Logo, $\mathcal{M} \cup\{Y\} \in U$, absurdo.

Logo, $\mathcal{M}$ é uma MAD family. Podemos aplicar o teorema anterior e obter $X \in \mathcal{I}^{*}(\mathcal{M})$ tal que para todo $n \in \omega, X \subseteq^{*} X_{n}$. Como $\mathcal{A} \subseteq \mathcal{I}$, segue a tese.

\subsection{Normalidade}

Nesta seção comentaremos brevemente sobre a normalidade de um espaço de Mrówka.

Proposição 2.51. Seja $\mathcal{A}$ uma almost disjoint family. Então $\psi(\mathcal{A})$ é normal se, e somente se, para todo $\mathcal{B} \subseteq \mathcal{A}$, os conjuntos $\mathcal{B}$ e $\mathcal{A} \backslash \mathcal{B}$ podem ser separados por abertos disjuntos. 
Demonstração. Se $\psi(\mathcal{A})$ é normal, então para todo $\mathcal{B} \subseteq \mathcal{A}$, os conjuntos $\mathcal{B}$ e $\mathcal{A} \backslash \mathcal{B}$ podem ser separados por abertos disjuntos pois estes dois conjuntos são fechados disjuntos.

Reciprocamente, suponha que para todo $\mathcal{B} \subseteq \mathcal{A}$, os conjuntos $\mathcal{B}$ e $\mathcal{A} \backslash \mathcal{B}$ podem ser separados por abertos disjuntos. Sejam $F, K \subseteq \Psi(\mathcal{A})$ fechados disjuntos. Seja $\mathcal{B}=F \cap \mathcal{A}$. Por hipótese, existem $U, V$ abertos disjuntos tais que $\mathcal{B} \subseteq U, \mathcal{A} \backslash \mathcal{B} \subseteq V$. Considere os abertos $(U \backslash K) \cup(F \cap \omega)$ e $(V \backslash F) \cup(K \cap \omega)$, que são disjuntos e contém respectivamente $F, K$.

Isso motiva a seguinte definição:

Definição 2.13. Seja $\mathcal{A}$ uma almost disjoint family. Dizemos que $X \subseteq \omega$ separa $\mathcal{B}, C \subseteq \mathcal{A}$ se para todo $A \in \mathcal{B}, A \subseteq^{*} X$ e para todo $A \in \mathcal{C}, A \cap X={ }^{*} \emptyset$. Dados $\mathcal{B}, C$, se existe $X$ como antes, então dizemos que $\mathcal{B}, C$ podem ser separados.

Isso se relaciona com a noção de normalidade da seguinte forma:

Proposição 2.52. Seja $\mathcal{A}$ uma almost disjoint family e $\mathcal{B}, \mathcal{C} \subseteq \mathcal{A}$. Então $\mathcal{B}, C$ podem ser separados se, e somente se, em $\Psi(\mathcal{A})$ podem ser separados por abertos.

Demonstração. Suponha que $\mathcal{B}, \mathcal{C}$ podem ser separados e tome $X$ tal que para todo $A \in \mathcal{B}, A \subseteq * X$ e para todo $A \in \mathcal{C}, A \cap X={ }^{*} \emptyset$. Seja $U=X \cup\left\{A \in \mathcal{A}: A \subseteq \subseteq^{*} X\right\}$ e $V=(\omega \backslash X) \cup\left\{A \in \mathcal{A}: A \cap X={ }^{*} \emptyset\right\}$. Temos que $U, V$ são abertos, $\mathcal{B} \subseteq U, \mathcal{C} \subseteq V$.

Reciprocamente, suponha que $\mathcal{B}, \mathcal{C}$ podem ser separados por abertos. Sejam $U, V$ abertos disjuntos com $\mathcal{B} \in U, \mathcal{C} \in V$. Seja $X=U \cap \omega$. Dado $A \in \mathcal{B}$, temos que $A \subseteq^{*} U$, logo $A \subseteq^{*} X$. Por outro lado, dado $A \in \mathcal{C}$, temos que $A \subseteq{ }^{*} V$, $\log o A \cap X={ }^{*} \emptyset$.

Se $\mathcal{A}$ é enumerável, então $\Psi(\mathcal{A})$ é enumerável e regular, e, portanto, normal. Agora daremos um exemplo de uma almost disjoint family cujo $\Psi$-espaço não é normal.

Teorema 2.53. Existe uma almost disjoint family $\mathcal{A}$ não enumerável tal que não existem duas subfamílias não enumeráveis disjuntas que podem ser separados.

Demonstração. Recursivamente, construiremos uma almost disjoint family $\mathcal{A}=\left\{A_{\alpha}: \alpha<\omega_{1}\right\}$ tal que:

$$
\forall \alpha<\omega_{1} \forall n \in \omega\left|\left\{\beta<\alpha: A_{\alpha} \cap A_{\beta} \subseteq n\right\}\right|<\omega .
$$

$\mathrm{Na}$ verdade, a seguinte propriedade, que é mais forte que a anterior, será satisfeita:

$$
\forall \alpha<\omega_{1} \forall n \in \omega\left|\left\{\beta<\alpha:\left|A_{\alpha} \cap A_{\beta}\right| \leq n\right\}\right|<\omega .
$$

Para fazer isso, seja $\left\{A_{n}: n \in \omega\right\}$ uma partição infinita de $\omega$ por subconjuntos infinitos. Suponha que $A_{\beta}$ foi escolhido para todo $\beta<\alpha$ para algum algum $\alpha<\omega_{1}$ infinito. Enumeremos $\left\{A_{\beta}: \beta<\alpha\right\}=\left\{B_{n}: n \in \omega\right\}$. Para cada $n \in \omega$, seja $a_{n} \subseteq B_{n} \backslash \bigcup_{j<n} B_{j}$ de tamanho $n$. Seja $A_{\alpha}=\bigcup_{n \in \omega} a_{n}$. Assim, dado $n$ natural, se $m>n$ então $a_{m} \subseteq A_{\alpha} \cap B_{m}$, o que mostra que $\left|A_{\alpha} \cap B_{m}\right| \geq m>n$.

Afirmo que $\mathcal{A}$ tem a propriedade desejada. Se $X$ separa $\mathcal{B}, \mathcal{C}$, então existe $n \in \omega$ tal que $\mid\{A \in \mathcal{C}$ : $A \cap X \subseteq n\} \mid=\omega_{1}$. Seja $\mathcal{C}^{\prime}=\{A \in \mathcal{C}: A \cap X \subseteq n\}$. Existe $m \in \omega$ tal que $|\{A \in \mathcal{B}: A \backslash X \subseteq m\}|=\omega_{1}$. Seja $\mathcal{B}=\{A \in \mathcal{B}: A \backslash X \subseteq m\}$. Seja $k=\max \{m, n\}$. Temos que

$$
\left(\bigcup \mathcal{B}^{\prime}\right) \cap\left(\bigcup \mathcal{C}^{\prime}\right) \subseteq(m \cup X) \cap\left(\bigcup \mathcal{C}^{\prime}\right) \subseteq m \cup n=k
$$

Porém, existe $\alpha<\omega_{1}$ tal que $A_{\alpha} \in \mathcal{B}^{\prime}$ e existem infinitos $\beta<\alpha$ tais que $A_{\beta} \in \mathcal{C}^{\prime}$. Para cada um desses $\beta, A_{\alpha} \cap A_{\beta} \subseteq k$. Logo, $\left|\left\{\beta<\alpha: A_{\alpha} \cap A_{\beta} \subseteq k\right\}\right|=\omega$, o que é absurdo.

$\mathrm{Na}$ demonstração do teorema acima, construímos uma almost disjoint family de cardinalidade $\omega_{1}$ tal que

$$
\forall \alpha<\omega_{1} \forall n \in \omega\left|\left\{\beta<\alpha: A_{\alpha} \cap A_{\beta} \subseteq n\right\}\right|<\omega .
$$

Uma tal almost disjoint family possui um nome especial. Além disso, uma almost disjoint family que satisfaz o enunciado do Teorema acima também recebe um nome especial. 
Definição 2.14. Uma almost disjoint family $\mathcal{A}$ é dita de Luzin se existe uma enumeração injetora $\mathcal{A}=\left\{A_{\alpha}: \alpha<\omega_{1}\right\}$ tal que

$$
\forall \alpha<\omega_{1} \forall n \in \omega\left|\left\{\beta<\alpha: A_{\alpha} \cap A_{\beta} \subseteq n\right\}\right|<\omega .
$$

Uma almost disjoint family $\mathcal{A}$ é dita de Luzin* se existe uma enumeração injetora $\mathcal{A}=\left\{A_{\alpha}\right.$ : $\alpha<\omega_{1}$ tal que

$$
\forall \alpha<\omega_{1} \forall n \in \omega\left|\left\{\beta<\alpha:\left|A_{\alpha} \cap A_{\beta}\right| \leq n\right\}\right|<\omega .
$$

Uma almost disjoint family não enumerável é inseparável se não existem duas subfamílias não enumeráveis disjuntas que podem ser separadas.

Assim, da demonstração do teorema acima podemos abstrair o seguinte corolário:

Corolário 2.54. Existe uma almost disjoint family de Luzin*. Toda almost disjoint family de Luzin é inseparável. Toda almost disjoint family de Luzin* é de Luzin. 


\section{Capítulo 3}

\section{Espaços de funções}

\subsection{A propriedade de Lindelöf em um espaço de funções}

Nessa seção, seguimos vários elementos do artigo [DS06]. A pergunta principal é: quando que um espaço de funções contínuas é Lindelöf? Vamos começar a seção provando que sempre que $X$ possuir base enumerável, $C(X)$ é Lindelöf. Além destes exemplos, há na literatura poucos exemplos de espaços não metrizáveis cujos espaços de funções contínuas são Lindelöf ([DS06]). O objetivo desta seção é utilizar $\Psi$-espaços, além de alguns princípios combinatórios e hipóteses sobre cardinais, para encontrar mais alguns exemplos.

Definição 3.1. Um network em $X$ é uma coleção $\mathcal{N}$ de subconjuntos de $X$ tal que para todo $x \in X$ e toda vizinhança aberta $U$ de $X$ existe $N \in \mathcal{N} \operatorname{com} x \in N \subseteq U$. Define-se o peso de network de $X$ por:

$$
\operatorname{nw}(X)=\omega+\min \{|\mathcal{N}|: \mathcal{N} \text { é um network de } X)\}
$$

É claro que $\mathrm{nw}(\mathrm{X}) \leq \mathrm{w}(X)$. Dado um espaço topológico $X$, o grau de Lindelöf de $X$ é definido como $\mathrm{L}(X)=\min \left\{\kappa: \forall \mathcal{B}\left(\mathcal{B}\right.\right.$ é cobertura aberta de $X \rightarrow \exists \mathcal{C} \in[\mathcal{B}]^{\leq \kappa} \mathcal{C}$ é cobertura de $\left.X\right\}$.

Proposição 3.1. Para todo espaço $X, \mathrm{~L}(X) \leq \mathrm{nw}(X)$.

Demonstração. Seja $\mathcal{N}$ um network para $X$ de cardinalidade mínima. Seja $\mathcal{U}$ uma cobertura aberta para $X$. Seja $\mathcal{N}^{\prime}=\{N \in \mathcal{N}:(\exists U \in \mathcal{U})(N \subseteq U)\}$ Para cada $N \in \mathcal{N}^{\prime}$, tome $U_{N} \in \mathcal{U}$ com $N \subseteq U_{N}$. Considere $\mathcal{U}^{\prime}=\left\{U_{N}: N \in \mathcal{N}^{\prime}\right\}$. Temos que $\left|\mathcal{U}^{\prime}\right| \leq\left|\mathcal{N}^{\prime}\right| \leq|\mathcal{N}|=\operatorname{nw}(X)$ e $\mathcal{U}^{\prime} \subseteq \mathcal{U}$ é uma cobertura aberta, pois dado $x \in X$ existe $N \in \mathcal{N}$ com $x \in N$. Daí, $x \in U_{N}$.

Proposição 3.2. Para todo espaço $X$ e para todo espaço $Y, \operatorname{nw}\left(C_{p}(X, Y)\right) \leq \mathrm{nw}(X)$. w $(Y)$. Em particular, $\operatorname{nw}\left(C_{p}(X)\right) \leq \operatorname{nw}(X)$

Demonstração. Fixe uma base $\mathcal{B}$ de $Y$ de cardinalidade w $(Y)$. Seja $\mathcal{N}$ um network para $X$ de cardinalidade mínima. Para cada $N_{1}, \ldots, N_{k} \in \mathcal{N}$ e para cada $I_{1}, \ldots, I_{k} \in \mathcal{B}$, seja:

$$
W\left(\left(N_{1}, \ldots, N_{k}\right),\left(I_{1}, \ldots, I_{k}\right)\right)=\left\{f \in C_{p}(X): \forall i \leq k\left(f\left[N_{i}\right] \subseteq I_{i}\right)\right\}
$$

Seja $\mathcal{M}$ a coleção de todos os conjuntos desse tipo. Segue que $|\mathcal{M}| \leq \operatorname{nw}(X) \mathrm{w}(Y)$. Seja $g \in$ $C_{p}(X)$ e um aberto básico contendo $g$ da forma:

$$
U=\left\{f \in C_{p}(X): \forall i \leq l\left(f\left(x_{i}\right) \in J_{i}\right)\right\}
$$

para alguns $x_{1}, \ldots, x_{l}$ fixados e $J_{1}, \ldots, J_{l} \in \mathcal{B}$ fixados. $U$.

Para cada $i$, existe $N_{i}$ tal que $x_{i} \in N_{i} \subseteq g^{-1}\left[J_{i}\right]$. Temos que $g \in W\left(\left(N_{1}, \ldots, N_{l}\right),\left(J_{1}, \ldots, J_{l}\right)\right) \subseteq$ 
Corolário 3.3. Dados espaços topológicos $X$ e $Y$, temos que

$$
\mathrm{L}\left(C_{p}(X, Y)\right) \leq \mathrm{nw}\left(C_{p}(X, Y)\right) \leq \mathrm{nw}(X) . \mathrm{w}(Y) \leq \mathrm{w}(X) . \mathrm{w}(Y) .
$$

Em particular, se $X$ e $Y$ possuem bases enumeráveis, então $C_{p}(X, Y)$ é Lindelöf.

Utilizaremos $\Psi$-espaços para construir exemplos de espaços sem base enumerável cujos espaços de funções são, ou não, Lindelöf.

Proposição 3.4. Seja $\mathcal{A}$ uma MAD family. Então $X=C_{p}(\Psi(\mathcal{A}))$ não é Lindelöf.

Demonstração. Para cada $A \in \mathcal{A}$, seja $V_{A}=\{f \in X: f(A) \neq 0\}$. Para $k<m<\omega$, seja $V_{k, m}=\left\{f \in X:\right.$ se $k \leq n<m$ então $f(n)<\frac{1}{2}$ e $\left.f(m)<\frac{1}{1+k}\right\}$. Seja $\mathcal{V}=\left\{V_{A}: A \in \mathcal{A}\right\} \cup\left\{V_{k, m}\right.$ : $k<m<\omega\}$. Veremos que $\mathcal{V}$ é uma cobertura aberta de $X$ sem subcobertura enumerável.

Dada $f \in X$, ou existe $A \in \mathcal{A}$ com $f(A) \neq 0$, e, nesse caso, $f \in V_{A}$, ou $f(A)=0$ para todo $A \in \mathcal{A}$. Nesse segundo caso, afirmo que $\lim _{n \rightarrow \infty} f(n)$ existe e é 0 , pois se isso fosse falso, existiria $\epsilon>0$ tal que para todo $N$ existe $n>N$ com $|f(n)| \geq \epsilon$. Assim, consegue-se definir recursivamente uma sequência $b_{n}$ de números naturais estritamente crescente com $\left|f\left(b_{n}\right)\right| \geq \epsilon$. Existe $A \in \mathcal{A}$ tal que $B \cap A$ é infinito. Mas então, enumerando $B \cap A$ em ordem crescente, temos uma subsequência de $b_{n}, b_{n_{k}}$ tal que $f\left(b_{n_{k}}\right) \rightarrow 0$ (pela continuidade de $f$ ), absurdo. Assim, $\lim _{n \rightarrow \infty} f(n)=0$. $\operatorname{Logo}_{\text {, }}$ existe $k$ tal que para todo $n \geq k$, temos que $f(n)<\frac{1}{2}$, e existe $m>k$ com $f(n)<\frac{1}{k+1}$ para todo $n \geq m$. Então $f \in V_{k, m}$.

Seja $\mathcal{W} \subseteq \mathcal{V}$ enumerável. Existe $D \in \mathcal{A}$ com $V_{D} \notin \mathcal{W}$. Considere a seguinte função: $g(n)=1$ para todo $n \in D, g(D)=1, g(n)=\frac{1}{1+|D \cap n|}$ se $n \notin D$ e $g(B)=0$ se $B \in \mathcal{A} \backslash\{D\}$. Temos que $g$ é contínua em todo ponto de $\omega$ pois este é discreto, temos que $g$ é contínua em $D$ pois é constante em qualquer uma de suas vizinhanças básicas, e temos que se $B \in \mathcal{D} \backslash\{D\}, g$ é contínua em $B$ : dado $\epsilon>0$, basta tomar $n>0 \operatorname{com} \frac{1}{n}<\epsilon$, tomar $m \operatorname{com}|m \cap D|+1>n$, e então se $k \geq m$ temos que $f[\{B\} \cup(B \backslash m)] \subseteq(-\epsilon, \epsilon)$.

Temos que $g \notin V_{A}$ para todo $A$ com $V_{A} \in \mathcal{W}$. Suponha que $k<m<\omega$. Se existir $n$ com $k \leq n<m$ e $n \in A$, então $g(n)=1$, logo, $g \notin V_{k, m}$. Caso contrário, $A \cap m \subseteq k$. Se $m \in A$, temos que $g(m)=1$. Caso contrário, $g(m) \geq \frac{1}{1+k}$. Assim, $g \notin V_{k, m}$.

Assim, $X$ não é Lindelöf.

Vamos utilizar para alguns exemplos desta seção o princípio $\diamond$, que foi enunciado no primeiro capítulo (Definição 1.15).

Proposição 3.5. Assuma $\diamond$. Existe uma MAD family $\mathcal{A}$ tal que $C_{p}(\Psi(\mathcal{A}),\{0,1\})$ é Lindelöf.

Demonstração. Seja $X=C_{p}(\Psi(\mathcal{A}),\{0,1\})$. Construiremos uma MAD family $\left\{\mathcal{A}_{\alpha}: \omega \leq \alpha<\omega_{1}\right\}$ recursivamente.

Fixe uma enumeração $\left\{\phi_{\alpha}: \alpha<\omega_{1}\right\}$ de todas as funções de um subconjunto finito de $\omega_{1}$ em 2 .

Seja $\left\{M_{\alpha}: \alpha<\omega_{1}\right\}$ uma enumeração de $\mathscr{P}(\omega)$ de modo que cada subconjunto de $\omega$ apareça nela cofinalmente.

Para cada $\beta \operatorname{com} \omega+\omega \leq \beta<\omega_{1}$, seja $b_{\beta}: \omega \rightarrow \beta \backslash \omega$ bijeção. Finalmente, seja $\left(S_{\beta}: \omega+\omega \leq\right.$ $\left.\beta<\omega_{1}\right)$ uma sequência diamante.

Seja $\mathcal{A}_{\omega+\omega}$ uma partição infinita enumerável de subconjuntos infinitos de $\omega$, e escrevemos $\mathcal{A}_{\omega+\omega}=\left\{A_{\omega+n}: n \in \omega\right\}$.

Para cada passo $\beta$ com $\omega+\omega \leq \beta<\omega_{1}$, construiremos duas sequências estritamente crescentes de números naturais, $q_{\beta}(n)$ e $k_{\beta}(n)$. Consideraremos dois casos. Dado $\mathcal{B} \subseteq \mathcal{A}_{\beta}\left(=\left\{A_{\alpha}: \omega \leq \alpha<\beta\right\}\right)$ finito, um natural $l$ e $K \subseteq l$, seja $f_{(\mathcal{B}, l, K)}$ definida em $\Psi\left(\mathcal{A}_{\beta}\right)$ dado por $f(A)=1$ se $A \in \mathcal{B}, f(n)=1$ se $n \in K$ ou se $(n \in A \backslash l)$ para algum $A \in \mathcal{B}$. Caso contrário, $f(x)=0$.

Caso 1: Para todo $\alpha \in S_{\beta}$, $\operatorname{dom}\left(\phi_{\alpha}\right) \subseteq \beta$ e a família $\left\{V\left(\phi_{\alpha}\right): \alpha<\beta\right\}$ recobre todas as funções $f_{(\mathcal{B}, l, K)}$ como acima, onde $V(\phi)=\left\{f \in C_{p}\left(\Psi\left(A_{\alpha}\right), 2\right): \forall k \in \omega \cap \operatorname{dom}(\phi)(f(k)=\phi(k)) \wedge \forall \alpha \in\right.$ $\left.\operatorname{dom}(\phi) \backslash \omega\left(f\left(A_{\alpha}\right)=\phi(\alpha)\right)\right\}$ 
Nesse caso, defina $q_{\beta}(0)=0$. Definido $q_{\beta}(n)$, seja:

$$
F_{n}^{\beta}=\left\{f_{(\mathcal{B}, l, K)}: \mathcal{B} \subseteq\left\{A_{\alpha}: \alpha \in b_{\beta}\left[q_{\beta}(n)\right]\right\}, l \leq q_{\beta}(n), K \subseteq l\right\}
$$

Temos que $F_{n}^{\beta}$ é finito. Para cada $f \in F_{n}^{\beta}$, seja $\alpha(f) \in S_{\beta} \operatorname{com} f \in V\left(\phi_{\alpha(f)}\right)$ e seja $q_{\beta}(n+1)$ o menor natural maior que $q_{\beta}(n)$ tal que para todo $f \in F_{n}^{\beta}$, $\operatorname{dom}\left(\phi_{\alpha(f)}\right) \cap \omega \subseteq q_{\beta}(n+1)$.

Caso 2: Caso contrário, seja $q_{\beta}(n)=n$ para todo $n$.

Re-enumeremos $\left\{A_{\alpha}: \omega \leq \alpha<\beta\right\}$ como $\left\{B_{n}: n \in \omega\right\}$. Nesse ponto, a sequências $k_{\alpha}$ para $\omega+\omega \leq \alpha<\beta$ já estão definidas. Tome $k_{\beta}$ de modo que para toda sequência $g$ em $\left\{q_{\beta}\right\} \cup\left\{k_{\alpha}\right.$ : $\omega+\omega \leq \alpha<\beta\}$, existe $j$ tal que para todo $n \geq j$ o conjunto $\left\{g(i): k_{\beta}(n)<g(i)<k_{\beta}(n+1)\right\}$ tem tamanho pelo menos $n$, e tal que $\left(\omega \backslash \bigcup_{i<n} B_{i}\right) \cap\left(k_{\beta}(n), k_{\beta}(n+1)\right) \neq \emptyset$ para todo $n$, o que é possível pois só temos uma quantidade enumerável de funções.

Para definir $A_{\beta}$, se existir uma sequência $\left(x_{n}: n \in \omega\right)$ de números naturais tal que

(a) para todo $n \in \omega, x_{n} \in \omega \backslash \bigcup_{i<n} A_{i}$,

(b) $\forall n \in \omega,\left|\left\{x_{n}: n \in \omega\right\} \cap k_{\beta}(n+1) \backslash k_{\beta}(n)\right| \leq 1$,

(c) $\left|\left\{x_{n}: n \in \omega\right\} \cap M_{\beta}\right|=\omega,\left|\left\{x_{n}: n \in \omega\right\} \backslash M_{\beta}\right|=\omega ;$

então seja $A_{\beta}=\left\{x_{n}: n \in \omega\right\}$. Caso contrário, seja $A_{\beta}=\left\{x_{n}: n \in \omega\right\}$, sendo $\left(x_{n}\right)_{n \in \omega}$ uma sequência que satisfaça apenas as duas primeiras condições. Para ver que uma tal sequência existe, seja $x_{0}=k_{\beta}(0)$, e, definido $x_{n}$, tome $x_{n+1} \in\left(\omega \backslash \bigcup_{i<n} A_{i}\right) \cap\left(k_{\beta}(n), k_{\beta}(n+1)\right) \neq \emptyset$. Dessa forma, a igualdade vale em (b).

Notemos que (a) implica que $A_{\beta}$ é infinito: se fosse finito, $\left(x_{n}: n \in \omega\right)$ teria uma subsequência constante, mas isso é impossível pois como Como $\left\{A_{\omega+n}: n \in \omega\right\}$ é uma partição de $\omega$, dado $n \in \omega$ existe $m \in \omega$ tal que $x_{n} \in A_{m}$, daí se $l \geq m$ temos que $x_{l} \neq x_{n}$. Temos que $A_{\beta}$ também implica que se $\omega \leq \alpha<\beta$ então $A_{\alpha} \cap A_{\beta}$ é finito: Existe $n$ tal que $A_{\alpha}=B_{n}$, daí $A_{\alpha} \cap A_{\beta} \subseteq\left\{x_{0}, \ldots, x_{n}\right\}$.

Procedendo dessa forma, $\mathcal{A}=\left\{A_{\alpha}: \omega \leq \alpha<\omega_{1}\right\}$ é uma almost disjoint family. Devemos ver que ela é maximal. Se não for, existe $X \subseteq \omega$ infinito tal que para todo $\beta, A_{\beta} \cap X$ é finito. Existe $\beta$ tal que $M_{\beta}=X$. Tome $x_{0} \in A_{0} \backslash M_{\beta}$. Escolhido $x_{n}$, se $n+1$ é par, tome $x_{n} \in A_{\frac{n+1}{2}} \backslash \bigcup_{i<\frac{n+1}{2}} A_{i}$ em algum $k_{\beta}(n+1) \backslash k_{\beta}(n)$ para o qual ainda não tenha sido escolhido nenhum elemento. Caso $x_{n}$ seja ímpar, tome $x_{n} \in M_{\beta} \backslash \bigcup_{i<\frac{n+1}{2}} A_{i}$ em algum $k_{\beta}(n+1) \backslash k_{\beta}(n)$. Dessa forma, conclui-se que $A_{\beta}$ foi escolhido de modo que $A_{\beta} \cap M_{\beta}^{2}$ é infinito, absurdo.

Agora seja $f: \Psi(\mathcal{A}) \rightarrow 2$ contínua. Veremos que $|\{A \in \mathcal{A}: f(A)=0\}|,|\{A \in \mathcal{A}: f(A)=1\}| \geq$ $\omega$ é impossível. Considere $X=\omega \cap f^{-1}[\{0\}]$. Então $X \subseteq \omega$ é tal que $\{A \in \mathcal{A}:|X \cap A|=\omega\}$ e $\{A \in \mathcal{A}:|A \backslash X|=\omega\}$ são infinitos. Então existe $\beta<\omega_{1} \operatorname{com} M_{\beta}=X$ e, como $M_{\beta}=X$ para infinitos $\beta$, também podemos supor que $\left\{A \in \mathcal{A}_{\beta}:|X \cap A|=\omega\right\}$ e $\left\{A_{\beta} \in \mathcal{A}:|A \backslash X|=\omega\right\}$ são infinitos. Nesse caso, $X_{\beta}$ foi escolhido de modo que vale (c) (provaremos abaixo). Assim, $A_{\beta} \cap X$ e $A_{\beta} \backslash X$ são infinitos e $f^{-1}[\{0\}] \supset X$ e $f^{-1}[\{1\}] \supset \omega \backslash X$, segue que $f$ é descontínua em $A_{\beta}$, absurdo.

Para ver que no passo $\beta$ era possível escolher $\left(x_{n}\right)_{n \in \omega}$ satisfazendo (c), sejam $A, B$ subconjuntos infinitos de $\omega$ disjuntos que não contém o 0 de modo que se $n \in A$ então $B_{n} \cap X$ é infinito, e se $n \in B$, então $B_{n} \backslash$ é infinito. fixe $x_{0}=k_{\beta}(0)$. Escolhido $x_{n}$, se $n+1 \in A$, tomemos $x_{n+1} \in$ $B_{n} \cap X \backslash \bigcup_{i \leq n} A_{i}$ maior que $k_{\beta}(j)$, onde $j$ é tal que $k_{\beta}(j)>x_{m}$ para todo $m \leq n$. Se $n+1 \in B$, tomemos $x_{n+1} \in B_{n} \backslash X \backslash \bigcup_{i \leq n} A_{i}$ maior que $k_{\beta}(j)$, onde $j$ é tal que $k_{\beta}(j)>x_{m}$ para todo $m \leq n$. Caso nenhum dos dois ocorra, tomemos $x_{n+1} \in \omega \backslash \bigcup_{i \leq n} A_{i}$ maior que $k_{\beta}(j)$, onde $j$ é tal que $k_{\beta}(j)>x_{m}$ para todo $m \leq n$.

Agora veremos que $X=C_{p}(\Psi(A), 2)$ é Lindelöf. Primeiro, notemos que $\left\{V_{\alpha}: \alpha<\omega_{1}\right\}$ é uma base de $X$, onde $V_{\alpha}=\left\{f \in X: \forall k \in \omega \cap \operatorname{dom}(\phi)(f(k)=\phi(k)) \wedge \forall \alpha \in \operatorname{dom}(\phi) \backslash \omega\left(f\left(A_{\alpha}\right)=\Psi(\alpha)\right)\right\}$. Dito isso, seja $\mathcal{V}$ uma cobertura aberta por abertos básicos. Podemos supor que $\mathcal{V}=\left\{V_{\alpha}: \alpha \in I\right\}$ para algum $I \subseteq \omega_{1}$.

Seja $C \subseteq \omega_{1}$ o conjunto dos ordinais enumeráveis $\beta$ que satisfazem:

(i) para cada $\alpha \in \beta \cap I, \operatorname{dom}\left(\phi_{\alpha}\right) \subseteq \beta$ ); 
(ii) Se $\mathcal{B}$ é subconjunto finito de $\mathcal{A}_{\beta}, l<\omega$ e $K \subseteq l$ então existe $\alpha \in \beta \cap I \operatorname{com} f_{(\beta, l, K)} \in V_{\alpha}$.

$C$ é fechado: Suponha que $\gamma<\omega_{1}$ é um ordinal limite e $\gamma \cap C$ é ilimitado em $\gamma$. As propriedades (i) e (ii) para $\gamma$ seguem imediatamente.

$C$ é ilimitado: dado $\mu \in \omega_{1}$, seja $g(\mu)$ o primeiro ordinal maior que $\mu$ que satisfaz $\forall \alpha \in \mu \cap$ $\left.I\left(\operatorname{dom}\left(\phi_{\alpha}\right) \subseteq g(\mu)\right)\right)$ e $\forall \mathcal{B} \in\left[\mathcal{A}_{\mu}\right]^{<\omega} \forall l<\omega \forall K \subseteq l \exists \alpha \in g(\mu) \cap I\left(f_{(\beta, l, K)} \in V_{\alpha}\right)$. Temos que $\mu<\sup \left\{g^{n}(\mu): n \in \omega\right\}$ e esse último está em $C$.

Temos que $S=\left\{\beta \in \omega_{1}: S_{\beta}=I \cap \beta\right\}$ é estacionário em $\omega_{1}$, então tome $\beta \in C \cap S$. Temos que $\mathcal{W}=\left\{V_{\alpha}: \alpha \in S_{\beta}\right\}$ é um subconjunto enumerável de $\mathcal{V}$. Veremos que $\mathcal{W}$ recobre as funções contínuas que atingem 1 em elementos de $\mathcal{A}$ apenas em uma quantidade finita vezes. De forma análoga, é possível provar que existe uma subcobertura enumerável que recobre funções as que atingem 0 por elementos de $\mathcal{A}$ apenas uma quantidade finita de vezes, o processo é análogo, e vimos que uma das duas coisas deve necessariamente ocorrer. Isso implica que o espaço é Lindelöf.

Para isso, tome $f$ uma tal função contínua arbitrária. Seja $\mathcal{B}=\left\{A \in \mathcal{A}_{\beta}: f(A)=1\right\}$. Seja $\mathcal{D}=\left\{A \in \mathcal{A} \backslash \mathcal{A}_{\beta}: f(A)=1\right\}$. O conjunto $\mathcal{B} \cup \mathcal{D}$ é finito. Se $\mathcal{D}=\emptyset$, tome $l^{\prime}<\omega$ tal que se $n \in A$ e $n>l^{\prime}$ então $f(n)=1$. Agora tome $l>l^{\prime}$ tal que se $n \geq l$ então $f(n)=1 \rightarrow \exists A \in \mathcal{B}(n \in A)$. Tal $l$ existe, caso contrário teremos uma sequência $x_{n}$ disjunta de todo elemento de $\mathcal{B}$ estritamente crescente com $f\left(x_{n}\right)=1$. Pela maximalidade de $\mathcal{A}$, existe $A \in \mathcal{A}$ com $\left|A \cap\left\{x_{n}: n \in \omega\right\}\right|=\omega$. Temos por continuidade que $A \in \mathcal{D}$, absurdo. Logo, tomando tal $l$ e pondo $K=l \cap f^{-1}[\{1\}$, segue que $f=f_{(\mathcal{B}, l, K)}$ e por (ii) segue que $f \in \bigcup \mathcal{W}$.

Se $\mathcal{D} \neq \emptyset$ com $|\mathcal{D}|=m>0$, escrevemos $\mathcal{D}=\left\{A_{\gamma(1)}, \ldots, A_{\gamma(m)}\right\} \operatorname{com} \gamma(i) \geq \beta$.

Por construção, se $\gamma \geq \beta, n<\omega$, que $\left|A_{\gamma} \cap k_{\gamma}(n+1) \backslash k_{\gamma}(n)\right| \leq 1$. Além disso, existe $j=j(\gamma)$ tal que $\left|\left\{k_{\beta}(i): k_{\gamma}(n)<k_{\beta}(i)<k_{\gamma}(n+1)\right\}\right| \geq n$ e $\left|\left\{q_{\beta}(i): k_{\gamma}(n)<k_{\beta}(i)<k_{\gamma}(n+1)\right\}\right| \geq n$ para todo $n \geq j$. Então, se $j>j\left(\gamma_{1}\right), \ldots, j\left(\gamma_{n}\right), 2$ e $k_{\beta}(i)>k_{\gamma(1)}(j), \ldots, k_{\gamma(m)}(j)$, então para todo $A \in \mathcal{D},\left|A \cap k_{\beta}(i+1) \backslash k_{\beta}(i)\right| \leq 2$. para ver isso, dado $A_{\gamma} \in \mathcal{D}$, temos que $k_{\gamma}(j)<k_{\beta}(i)<k_{\beta}(i+1)$ e $k_{\gamma}(j)<k_{\gamma}(j+1)$. Se $k_{\gamma}(j)<k_{\beta}(i)<k_{\beta}(i+1) \leq k_{\gamma}(j+1)$, temos que a interseção tem no máximo um elemento. Caso $k_{\gamma}(j)<k_{\beta}(i) \leq k_{\gamma}(j+1)<k_{\beta}(i+1)$, segue que $k_{\gamma}(j)<k_{\beta}(i) \leq k_{\gamma}(j+1)<$ $k_{\beta}(i+1)<k_{\gamma}(j+2)$ e temos no máximo dois elementos. Finalmente, se $k_{\gamma}(j)<k_{\gamma}(j+1)<$ $k_{\beta}(i)<k_{\beta}(i+1)$, novamente segue que $k_{\gamma}(j)<k_{\gamma}(j+1)<k_{\beta}(i)<k_{\beta}(i+1)<k_{\gamma}(j+2)$ e temos no máximo dois elementos.

Temos que o conjunto $(\bigcup \mathcal{B}) \cap(\bigcup \mathcal{D})$ é finito. Tome $p^{\prime}$ maior que seu máximo tal que se $n \geq p^{\prime}$ e $n \in \bigcup B \cup \bigcup D$, então $f(n)=1$. Tome $p \geq p^{\prime}$ tal que se $n \geq p$ e $f(n)=1$ então $f(n) \in \bigcup \mathcal{B} \cup \bigcup \mathcal{D}$. Temos que $p$ existe pois caso contrário teríamos uma sequência estritamente crescente $x_{n}$ disjunta de todo elemento de $\mathcal{B} \cup \mathcal{D}$.

Tome $r \in \omega$ tal que $b_{\beta}[r] \supset\left\{\alpha<\beta: A_{\alpha} \in \mathcal{B}\right\}$.

Tome $n$ com $n>2 m+1, k_{\beta}(n)>\max \left\{k_{\gamma(1)}(j), \ldots, k_{\gamma(m)}(j)\right\}, k_{\beta}(n) \geq r, p$ e com $\mid\left\{q_{\beta}(i)\right.$ : $\left.k_{\beta}(n)<q_{\beta}(i)<k_{\beta}(n+1)\right\} \mid \geq n$ para todo $i$. O número de intervalos $q_{\beta}(i+1) \backslash q_{\beta}(i)$ que está contido no intervalo $k_{\beta}(n+1) \backslash k_{\beta}(n)$ é maior que $2 m$, mas só há $m$ conjuntos em $\mathcal{D}$, cada um intersectando $D$ em no máximo 2 pontos. Consequentemente, existe $\tilde{i} \operatorname{com} k_{\beta}(n)<q_{\beta}(\tilde{i})<q_{\beta}(\tilde{i}+1)<k_{\beta}(n+1)$ com $A \cap q_{\beta}(\tilde{i}+1) \backslash q_{\beta}(\tilde{i})=\emptyset$ para todo $A \in \mathcal{D}$.

Seja $l=q_{\beta}(\tilde{i}), K=l \cap f^{-1}[\{1\}]$. Temos que $f_{(\mathcal{B}, l, K)} \in V_{\alpha}$ para algum $\alpha \in S_{\beta}$. Também temos que $f_{(\mathcal{B}, l, K)} \in F_{\tilde{i}}^{\beta}$. Assim, $\alpha$ pode ser escolhido $\operatorname{com} \operatorname{dom}\left(\phi_{\alpha}\right) \cap \omega \subseteq q_{\beta}(\tilde{i}+1)$. Vimos que não existe $A \in \mathcal{D}$ que intersecta $q_{\beta}(\tilde{i}+1) \backslash q_{\beta}(\tilde{i})$, então $f$ e $f_{(\mathcal{B}, l, K)}$ coincidem em $\operatorname{dom}\left(\phi_{a}\right)$. Logo, $f \in V_{\alpha}$.

No capítulo anterior, vimos que $\mathfrak{b}$ é o menor cardinal para o qual existe uma família de funções de $\omega^{\omega}$ ilimitada (na ordem $\leq^{*}$ ). Na proposição abaixo, utilizaremos a suposição de que $\mathfrak{b}>\omega_{1}$, o que vale, por exemplo, sob o Axioma de Martin com a negação da Hipótese do Contínuo, mas é falso, por exemplo, no modelo de Cohen (ver Apêndice B) ou assumindo a Hipótese do Contínuo.

Proposição 3.6. Suponha que $\mathfrak{b}>\omega_{1}$. Então para toda MAD family $\mathcal{A}, X=C_{p}(\Psi(\mathcal{A}), 2)$ não é Lindelöf. 
Demonstração. Seja $\left\{A_{\alpha}: \alpha<\omega_{1}\right\}$ uma enumeração de algum subconjunto de $\mathcal{A}$ de cardinalidade $\omega_{1}$. Para cada $\alpha<\omega_{1}$, seja $e_{\alpha}$ uma bijeção estritamente crescente entre $\omega$ e $A_{\alpha}$. Como $\mathfrak{b}>\omega_{1}$, existe $f \in \omega^{\omega}$ dominando todas as $e_{\alpha}$. Definindo outra função recursivamente se necessário, podemos supor que $f$ é estritamente crescente.

Seja $g \in \omega^{\omega}$ definida recursivamente por $g(0)=f(0)$, e, definida $g(n)$, seja $g(n+1)=f(g(n)+1)$. Então, para cada $\alpha<\omega_{1}$, existe $j_{\alpha}$ natural tal que para todo $n \geq j_{\alpha}, A_{\alpha} \cap g(n+1) \backslash g(n) \neq \emptyset$ (pois $j_{\alpha} \leq n \leq g(n) \leq e_{\alpha}((g(n)) \leq f(g(n))<f(g(n)+1)=g(n+1)$.

Seja $j_{0}$ o menor $j \in \omega$ tal que $j=j_{\alpha}$ para uma quantidade não enumerável de $\alpha \in \omega_{1}$ e seja $\mathcal{B}=\left\{A_{\alpha}: \alpha<\omega_{1}, j(\alpha)=j_{0}\right\}$. Considere a cobertura $\mathcal{V}$ dos:

$V_{A}=\{f \in X: f(A)=1\}$ para $A \in \mathcal{A}, V(K)=\{f \in X: f(n)=1 \forall n \in K, f(n)=0 \forall n \in$ $g(m(K)) \backslash K\}$, onde $K \subseteq \omega$ é finito e $m(K)=j_{0}+1$ se $\max K<j_{0}$, e, caso contrário, $m(K)=j+1$ para o menor $j$ tal que $j_{0} \leq \max K<j$.

Para ver que $\mathcal{V}$ é uma cobertura de $X$, fixe $f \in X$. Se existe $A \in \mathcal{A}$ com $f(A)=1$, então $f \in V_{A}$. Caso contrário, pela maximalidade de $\mathcal{A}, K=f^{-1}[\{1\}]$ é finito, e, então $f \in V(K)$.

Se $\mathcal{W} \subseteq \mathcal{V}$ é enumerável, então existe $\alpha \operatorname{com} V_{A_{\alpha}} \notin \mathcal{W}$. Seja $B=A_{\alpha}$ e considere $h$ a função dada por $h(x)=1 \leftrightarrow x \in B$ ou $x=B$. Temos que $h$ é contínua. Temos que $h \notin V_{A}$ para todo $A \in \mathcal{W}$ e dado $K \subseteq \omega$ finito, temos que $h(n)=1$ para algum $n \in g(m(K)) \backslash g(m(K)-1)$, logo $h \notin V(K)$.

Proposição 3.7. Suponha que $\mathfrak{b}>\omega_{1}$. Se $\mathcal{A}$ é uma almost disjoint family de cardinalidade $\omega_{1}$, então $C_{p}(\Psi(\mathcal{A}))$ não é Lindelöf. Se, adicionalmente, $2^{\omega}<2^{\omega_{1}}$, então $C_{p}(\Psi(\mathcal{A}),\{0,1\})$ não é normal.

Demonstração. Sabemos que $\Psi(\mathcal{A})$ é separável. Temos que $\mathcal{A} \subseteq \Psi(\mathcal{A})$ é fechado, discreto e tem cardinalidade $\omega_{1}$.

Caso 1: $\Psi(\mathcal{A})$ não é normal.

Nesse caso, provaremos que o subespaço fechado $X=C_{p}(\Psi(\mathcal{A}),\{0,1\})$ de $C_{p}(\Psi(\mathcal{A}))$ não é Lindelöf.

Como $\omega$ é aberto, existem $\mathcal{B}, \mathcal{C} \subseteq \mathcal{A}$ fechados e disjuntos que não podem ser separados por abertos.

Observemos que se $\mathcal{A}_{0} \subseteq \mathcal{A}$ é enumerável, existe $f: \Psi(\mathcal{A}) \rightarrow\{0,1\}$ contínua separando $\mathcal{A}_{0}$ de $\mathcal{A} \backslash \mathcal{A}_{0}$. Se $\mathcal{A}_{0}$ for finito, tomemos $f$ tal que $f(A)=0$ para todo $A \in \mathcal{A}_{0}, f(n)=0$ para todo $n \in \bigcup \mathcal{A}_{0}$ e que vale $1 \mathrm{em}$ todos os outros pontos. Se $\mathcal{A}_{0}$ for infinito enumerável, escreva sem repetição $\mathcal{A}_{0}=\left\{A_{n}: n \in \omega\right\}$ e $A_{n}=\left\{a_{n, k}: k \in \omega\right\}$ em ordem crescente. Para cada $A \in \mathcal{A} \backslash \mathcal{A}_{0}$, seja $f_{A} \in \omega^{\omega}$ dado por:

$$
f_{A}(n)=\min \left\{k \in \omega: A \cap A_{n} \subseteq\left\{a_{n, i}: i<k\right\}\right\}
$$

Como $b>\omega_{1}$, seja $g \in \omega^{\omega}$ que domina cada $f_{A} \in \mathcal{A} \backslash \mathcal{A}_{0}$. Seja $h: \Psi(\mathcal{A}) \rightarrow\{0,1\}$ dada por $h(A)=1$ para todo $A \in \mathcal{A}_{0}, h\left(a_{n, k}\right)=1$ para todo $n \in \omega$ e $k \geq g(n) \operatorname{com} a_{n, k} \notin \bigcup_{i<n} A_{i}$, e $h(x)=0$ para todos os outros pontos. Temos que $h$ é contínua e separa $\mathcal{A}_{0}$ de $\mathcal{A} \backslash \mathcal{A}_{0}$.

Pela nossa observação, tanto $\mathcal{B}$ como $\mathcal{C}$ têm cardinalidade $\omega_{1}$. Escrevamos $\mathcal{B}=\left\{B_{\alpha}: \alpha<\omega_{1}\right\}$ e $\mathcal{C}=\left\{C_{\alpha}: \alpha<\omega_{1}\right\}$

Para $\alpha<\omega_{1}$, sejam:

$$
\begin{gathered}
U_{\alpha}=\left\{f \in X: f\left(B_{\alpha}\right)=0\right\} \\
V_{\alpha}=\left\{f \in X: f\left(B_{\alpha}\right)=f\left(C_{\alpha}\right)=1\right\}
\end{gathered}
$$

Seja $\mathcal{V}$ a coleção desses abertos. Temos que $\mathcal{V}$ forma uma cobertura de $X$, pois dada $f \in X$, se $f\left(B_{\alpha}\right)=1$ para todo $\alpha$, então pela escolha de $\mathcal{B}$ e $\mathcal{C}$ existe $\alpha \in \mathcal{C}$ tal que $f\left(B_{\alpha}\right)=f\left(C_{\alpha}\right)=1$.

Temos que se $\mathcal{W} \subseteq \mathcal{V}$ é enumerável, então $\mathcal{W} \subseteq\left\{U_{\alpha}, V_{\alpha}: \alpha<\beta\right\}$ para algum $\beta<\omega_{1}$, e aplicando novamente a observação, obtém-se $f \in X \operatorname{com} f\left(B_{\alpha}\right)=1$ e $f\left(C_{\alpha}\right)=0$ para todo $\alpha<\beta$. Assim, $f \notin \bigcup \mathcal{W}$.

Caso 2: $\Psi(\mathcal{A})$ é normal. 
Agora veremos que o subespaço fechado $Y=C_{p}(\Psi(A), \omega) \subseteq C_{p}(\Psi(A))$ não é Lindelöf. Enumeremos $\mathcal{A}=\left\{A_{\alpha}: \alpha<\omega_{1}\right\}$ para $\alpha<\beta<\omega_{1}$ e dado $n \in \omega$, seja

$$
U_{\alpha, \beta, n}=\left\{f \in Y: f\left(A_{\alpha}\right)=f\left(A_{\beta}\right)=n\right\}
$$

Seja $\mathcal{V}$ a coleção desses abertos. Temos que $\mathcal{V}$ é uma cobertura. Seja $\mathcal{W} \subseteq \mathcal{V}$ enumerável e seja

$$
\gamma=\sup \left\{\beta \in \omega_{1}: \exists \alpha<\beta \exists n \in \omega U_{\alpha, \beta, n} \in \mathcal{W}\right\}+1
$$

Seja $b: \gamma \rightarrow \omega$ bijetora. Seja $g: \mathcal{A} \rightarrow \omega$ dada por $g\left(\mathcal{A}_{\alpha}\right)=b(\alpha)$ para $\alpha<\gamma$ e $g\left(A_{\alpha}\right)=0$ para $\gamma \leq \alpha<\omega_{1}$. Como $\mathcal{A}$ é discreto, $g$ é contínua, e pela normalidade de $\Psi(\mathcal{A})$, ela pode ser estendida de forma contínua à uma função $f$ definida neste último. Podemos supor que a imagem de $f$ está contida em $\mathbb{N}$ redefinindo se necessário que $\tilde{f}(x)=n \leftrightarrow f(x) \in\left(n-\frac{1}{2}, n+\frac{1}{2}\right]$. $f$ não está em nenhum elemento de $\mathcal{W}$, pois se $\beta<\gamma$, temos que $f\left(A_{\alpha}\right) \neq f\left(A_{\beta}\right)$, o que termina a prova.

Para a firmação final, se $2^{\omega}<2^{\omega_{1}}$, um espaço separável que tem um subconjunto fechado, discreto e não enumerável não é normal. Logo, $\Psi(\mathcal{A})$ não é normal, e a demonstração segue como no caso 1.

Para o resto da seção, denotaremos por $\mathcal{T}=2^{\omega} \cup 2^{<\omega}$ o espaço topológico tal que $2^{<\omega}$ é discreto e tal que um aberto básico de $f \in 2^{\omega}$ é da forma $\{f \mid n: k \leq n \in \omega\} \cup\{f\}$ para $k \in \omega$. É imediato que $\mathcal{T}$ é homeomorfo a um $\Psi$-espaço sobre o conjunto infinito e enumerável $2^{<\omega}$. A letra $\mathcal{T}$ vem da palavra Tree (árvore). Além disso, denotaremos por $\mathcal{C}$ (que vem da palavra cone), o mesmo conjunto, de modo que $2^{<\omega}$ seja discreto e se $f \in{ }^{\omega} 2$, um aberto básico contendo $f$ é um conjunto do tipo $\{g \in \mathcal{C}: f \mid n \subseteq g\}$ para algum $n$ natural. É possível verificar facilmente que a topologia $\mathcal{T}$ é mais fina que a topologia $\mathcal{C}$. Apenas com a finalidade de estabelecer uma notação, seja $\Sigma=2^{<\omega}$

Lema 3.8. O espaço $C_{p}(\mathcal{T})$ não é Lindelöf.

Demonstração. Para $n \in \omega$, seja $V_{n}=\left\{f \in C_{p}(\mathcal{T}): \forall p \in{ }^{n} 2(f(p) \in(-1,1))\right\}$. Para $x \in{ }^{\omega} 2$, seja $V_{x}=\left\{f \in C_{p}(\mathcal{T}): f(x) \neq 0\right\}$. Para $A=\{p, q\} \subseteq 2^{<\omega}$, tal que $p \nsubseteq q$ e $q \nsubseteq p$, seja $V_{A}=\left\{f \in C_{p}(\mathcal{T}): f(p) \neq 0\right.$ e $\left.f(q) \neq 0\right\}$. Seja $\mathcal{V}$ a coleção dos abertos explicitados neste parágrafo.

Vejamos que $\mathcal{V}$ é uma cobertura. Fixada $f \in C_{p}(\mathcal{T})$, temos uma das situações abaixo:

- Existe $n \in \omega$ tal que para todo $p \in{ }^{n} 2$, temos que $f(p) \in(-1,1)$, e, nesse caso, $f \in V_{n}$,

- Para todo $n \in \omega$ temos que existe $p \in{ }^{n} 2 \operatorname{com}|f(p)| \geq 1$, e existem $p, q \in 2^{<\omega}$ tais que $|f(p)|,|f(q)| \geq 1$ um não contido no outro. Então $f \in V_{p, q}$.

- Para todo $n \in \omega$, existe $p \in{ }^{n} 2$ com $|f(p)| \geq 1$, e para todos $p, q$ dessa forma, $p \subseteq q$ ou $q \subseteq p$. Nesse caso, dado $n \in \omega$ existe um único $p_{n} \in{ }^{n} 2$ tal que $\left|f\left(p_{n}\right)\right| \geq 1$ e $x=\bigcup_{n \in \omega} p_{n} \in{ }^{\omega} 2$. Por continuidade, $|f(x)| \geq 1$, logo, $f \in V_{x}$.

Se $\mathcal{W} \subseteq \mathcal{V}$ é enumerável, existe $x \in{ }^{\omega} 2$ tal que $V_{x} \neq \mathcal{W}$. Definindo-se $f(p)=1$ para $p \subseteq x$ e $f(p)=0$ caso contrário, obtemos uma função contínua fora de $\bigcup \mathcal{W}$. Com efeito, $f$ é contínua pois $\{x\} \cup\{x \mid n: n \in \omega\}$ é clopen, $f \notin V_{n}$ para $n$ natural pois $f(x \mid n)=1$, se $p, q \in 2^{<\omega}$ e $f(p), f(q) \neq 0$ temos que $p, q$ são compatíveis, e, finalmente, dado $y \in{ }^{\omega} 2 \operatorname{com} y \neq x$, temos que $f(y)=0$.

Proposição 3.9. Assuma $\diamond$. Então existe $Z \subseteq{ }^{\omega} 2$ não enumerável tal que $C_{p}(Z \cup \Sigma)$ é Lindelöf.

Demonstração. Seja $X=2^{\leq \omega}$. Consideraremos as duas topologias sobre $X$ explicitadas nessa seção, $\mathcal{T}$ e $\mathcal{C}$.

Dado $\epsilon>0$ e $f: \Sigma \rightarrow \mathbb{R}$, seja $\operatorname{Osc}(f, \epsilon, \mathcal{T})$ o conjunto de todos os $x \in 2^{\omega}$ tal que para toda $\mathcal{T}$-vizinhança $U$ de $x$, existem $p, q \in U \cap \Sigma$ tal que $|f(p)-f(q)| \geq \epsilon$. Define-se então $\operatorname{Osc}(f, \mathcal{T})=$ $\bigcup_{\epsilon>0} \operatorname{Osc}(f, \epsilon, \mathcal{T})$. Analogamente, define-se $\operatorname{Osc}(f, \epsilon, \mathcal{C})$ e $\operatorname{Osc}(f, \mathcal{C})$.

Note que cada $\operatorname{Osc}(f, \epsilon, \mathcal{C}) \subseteq 2^{\omega}$ é fechado em $2^{\omega}$ na topologia produto: Suponha que $x \in 2^{\omega} \backslash$ $\operatorname{Osc}(f, \epsilon, \mathcal{C})$. Então existe $U$ vizinhança de $x$ em $\mathcal{C}$ tal que se $p, q \in \Sigma \cap U$ temos que $|f(p)-f(q)|<\epsilon$. 
Podemos supor que $U=\{y \in X: x \mid n \subseteq y\}$. Considere $V=\left\{z \in 2^{\omega}: z|n=x| n\right\}$. Temos que $V \subseteq 2^{\omega}$ é vizinhança aberta de $x$ e se $z \in V$, temos que $z \in U$, e se $p, q \in \Sigma \cap U$ temos que $|f(p)-f(q)|<\epsilon, \log 0, V \subseteq 2^{\omega} \backslash \operatorname{Osc}(f, \epsilon, \mathcal{C})$. Dessa forma, $\operatorname{Osc}(f, \mathcal{C})=\bigcup_{n \in \omega} \operatorname{Osc}\left(f, \frac{1}{n+1}, \mathcal{C}\right)$ é um $F_{\sigma}$ e vale que $\operatorname{Osc}(f, \mathcal{T}) \subseteq \operatorname{Osc}(f, \mathcal{C})$, pois a topologia $\mathcal{T}$ é mais fina que $\mathcal{C}$.

Como $\diamond$ implica a hipótese do contínuo, podemos enumerar $\mathbb{R}^{\Sigma}=\left\{f_{\alpha}: \alpha<\omega_{1}\right\}$. Seja $\mathcal{B}$ uma base enumerável para $\mathbb{R}$. A coleção dos seguintes conjuntos forma uma base para $\mathbb{R}^{X}$ :

$$
\left.V\left(\left(x_{0}, \ldots, x_{k}\right),\left(B_{0}, \ldots, B_{k}\right)\right)=\left\{f \in \mathbb{R}^{X}:(\forall i \leq k) f\left(x_{i}\right) \in \mathcal{B}_{i}\right)\right\}
$$

Onde $x_{0}, \ldots, x_{k} \in X$ e $B_{0}, \ldots, B_{k} \in \mathcal{B}$. Enumeremos essa base como $\left\{V_{\alpha}: \alpha<\omega_{1}\right\}$ e fixemos uma sequência $\diamond$ dada por $\left(S_{\alpha}: \alpha<\omega_{1}\right)$. Define-se:

$$
\mathcal{V}_{\alpha}=\left\{V_{\beta}: \beta \in S_{\alpha}\right\} .
$$

Agora, recursivamente, escolheremos, para $\beta<\omega_{1}$, pontos $x_{\beta} \in 2^{\omega}$, magros $M_{\beta} \subseteq 2^{\omega}$ e funções $g_{\beta}: \Sigma \rightarrow \mathbb{R}$ como se segue: Supondo que esses objetos foram escolhidos para todo $\beta<\alpha<\omega_{1}$, se existir $g: \Sigma \rightarrow \mathbb{R}$ satisfazendo:

(i) $\operatorname{Osc}(g, \mathcal{T}) \cap\left\{x_{\beta}: \beta<\alpha\right\}=\emptyset$

(ii) $\operatorname{Osc}(g, \mathcal{C}) \subseteq 2^{\omega}$ é magro.

(iii) Existe uma extensão $\mathcal{T}$-contínua de $g$ para $2^{\omega} \backslash \operatorname{Osc}(g, \mathcal{T})$ fora de $\bigcup \mathcal{V}_{\alpha}$.

Então seja $g=g_{\alpha}$ e $M_{\alpha}=\operatorname{Osc}(g, \mathcal{C})$, caso contrário, seja $g_{\alpha}$ a função nula e seja $M_{\alpha}=\emptyset$.

Se existir $x \in 2^{\omega} \backslash \bigcup_{\beta \leq \alpha} M_{\beta}$ tal que $x \notin\left\{x_{\beta}: \beta<\alpha\right\}$ tal que $f_{\alpha}$ não pode ser $\mathcal{T}$-estendida continuamente para $x$, seja esse ponto $x_{\alpha}$. Caso contrário, fixe $x_{\alpha} \in 2^{\omega} \backslash \bigcup_{\beta<\alpha} M_{\beta}$ tal que $x \notin\left\{x_{\beta}\right.$ : $\beta<\alpha\}$, o que é possível pelo teorema de Baire. Seja $Z=\left\{x_{\alpha}: \alpha<\omega_{1}\right\}$ e a indução está completa.

Afirmação: Seja $f \in \mathbb{R}^{\Sigma}$. Então ou $\operatorname{Osc}(f, \mathcal{C}) \subseteq 2^{\omega}$ é magro, ou para toda coleção enumerável $\left\{D_{n}: n \in \omega\right\}$ de subconjuntos nunca densos de $2^{\omega}$, o conjunto $\operatorname{Osc}(f, \mathcal{T}) \backslash \bigcup_{n \in \omega} D_{n}$ contém um conjunto perfeito.

Suponha que $\operatorname{Osc}(f, \mathcal{C}) \subseteq 2^{\omega}$ não é magro. Então existe $\epsilon>0$ tal que $\operatorname{Osc}(f, \epsilon, \mathcal{C})$ possui interior não vazio. Então existe $p_{\emptyset} \in \Sigma$ tal que $U_{\emptyset}=\left\{y \in 2^{\omega}: p_{\emptyset} \subseteq y\right\} \subseteq \operatorname{int}(\operatorname{Osc}(f, \epsilon, \mathcal{C})) \backslash D_{0}$.

Agora, suponha que escolhemos, para algum $\sigma \in 2^{n}$, para algum $n \in \omega$, um ponto $p_{\sigma} \in \Sigma$. Temos que os seguintes conjuntos são abertos disjuntos de $2^{\omega}$ :

$$
G_{0}=\left\{y \in 2^{\omega}: \widetilde{p_{\sigma}}(0) \subseteq y\right\}, G_{1}=\left\{y \in 2^{\omega}: \widetilde{p_{\sigma}}(1) \subseteq y\right\}
$$

Temos que como cl $D_{n}$ tem interior vazio, para $i=0,1$ existem $t_{i} \in \Sigma$ com $t_{i} \supset p_{\sigma}(i)$ tais que $W_{t_{i}}=\left\{y \in 2^{\omega}: t_{i} \subseteq y\right\} \subseteq G_{i}$ é disjunto de $D_{n}$. Como $W_{t_{0}}$ e $W_{t_{1}}$ são $\operatorname{subconjuntos} \operatorname{de} \operatorname{Osc}(f, \epsilon, \mathcal{C})$, para cada $i \in\{0,1\}$ existem $q_{i}, s_{i} \supset t_{i}$ em $\Sigma$ com $\left|f\left(q_{i}\right)-f\left(s_{i}\right)\right| \geq \epsilon$. Seja $p_{\sigma-(i)} \in\left\{q_{i}, s_{i}\right\}$ tal que $\left|f\left(p_{\sigma}\right)-f\left(p_{\sigma \frown(i)}\right)\right| \geq \frac{\epsilon}{2}$ e definamos $U_{\sigma \frown(i)}=\left\{y \in 2^{\omega}: p_{\sigma \frown(i)} \subseteq y\right\}$.

Considere $P=\left\{\bigcup_{n \in \omega} p_{\phi \mid n}: \phi \in 2^{\omega}\right\}$, e denotemos $p_{\phi}=\bigcup_{n \in \omega} p_{\phi \mid n}$. Temos $p_{\phi} \in U_{\phi \mid n}$, $\operatorname{logo}, p_{\phi} \notin D_{n}$. Temos que $P$ é fechado, sem pontos isolados e está contido em $\operatorname{Osc}(f, \mathcal{T})$. Para ver que $P$ não tem pontos isolados, dado $\phi$, temos que uma vizinhança básica de $p_{\phi}$ é do tipo $\left\{g \in 2^{\omega}: g\left|k=p_{\phi}\right| k\right\}$ para algum $k$. Tome $n$ tal que $p_{\phi \mid n} \supset p_{\phi} \mid k$. Tomando $\psi \in 2^{\omega}$ diferente de $\phi$ contendo $\phi \mid n$, temos que $p_{\psi}$ atesta que $p_{\phi}$ não é ponto isolado. Para ver que $P$ é fechado, basta ver que $P=\bigcap_{n \in \omega} \bigcup_{g \in 2^{n}} U_{p_{g}}$. Para ver que $p \subseteq \operatorname{Osc}(f, \mathcal{T})$, basta ver que para toda $\tau$-vizinhança $V$ de $p_{\phi}$ existe $n$ tal que $p_{\phi \mid n}$ e $p_{\phi \mid(n+1)}$ estão ambos em $V$, e temos que o módulo da diferença desses é $\geq \frac{\epsilon}{2}$.

Uma consequência da afirmação é que se $f \in C_{p}(\Sigma \cup Z)$ (na topologia de subespaço de $\mathcal{T}$ ), então o conjunto $\operatorname{Osc}(f \mid \Sigma, \mathcal{C})$ é magro. Com efeito, existe $\alpha$ tal que $f_{\alpha}=f \mid \Sigma$. A família $\left\{M_{\beta}: \beta \leq \alpha\right\}$ é uma coleção enumerável de subconjuntos magros de $2^{\omega}, \operatorname{logo}, \operatorname{se} \operatorname{Osc}\left(f_{\alpha}, \mathcal{C}\right)$ não fosse magro, 
pela afirmação e pela construção teríamos escolhido de modo que $f_{\alpha}$ não poderia ser estendida continuamente para $x_{\alpha}$, absurdo.

Agora veremos que $C_{p}(\Sigma \cup Z)$ é Lindelöf. Considere $\mathcal{V}$ uma cobertura aberta desse espaço. Podemos assumir que $\mathcal{V}=\left\{V_{\beta}: \beta \in I\right\}$ para algum $I \subseteq \omega_{1}$ (Originalmente, cada $V_{\beta}$ é um aberto de $\mathbb{R}^{X}$, mas podemos restringi-lo de forma natural para abertos de $\mathbb{R}^{\Sigma \cup Z}$ ). Temos que o conjunto $S=\left\{\alpha \in \omega_{1}:\left\{V_{\beta}: \beta \in I \cap \alpha\right\}=\mathcal{V}_{\alpha}\right\}$ é estacionário.

Para $k, n \in \omega, y_{0}, \ldots, y_{k} \in \Sigma \cup Z, G_{0} \ldots, G_{n}$ abertos básicos de $(X, \mathcal{C}), B_{0}, \ldots, B_{k}, B_{0}^{\prime}, \ldots, B_{n}^{\prime} \in$ $\mathcal{B}$, seja:

$K\left(\left(y_{i}\right)_{i=1}^{k},\left(G_{j}\right)_{i=1}^{n},\left(B_{i}\right)_{i=1}^{l},\left(B_{j}^{\prime}\right)_{j=1}^{n}\right)=\left\{f \in C_{p}(\Sigma \cup Z):(\forall i \leq k) f\left(y_{i}\right) \in B_{i}\right.$ e $\left.(\forall j \leq n) f\left[G_{j}\right] \subseteq B_{j}^{\prime}\right\}$

Seja $C \subseteq \omega_{1}$ o conjunto dos ordinais enumeráveis $\alpha$ tais que para $k, n \in \omega, y_{0}, \ldots, y_{k} \in \Sigma \cup$ $Z, G_{0} \ldots, G_{n}$ abertos básicos de $(X, \mathcal{C}), B_{0}, \ldots, B_{k}, B_{0}^{\prime}, \ldots, B_{n}^{\prime} \in \mathcal{B}$, se $\left\{y_{0}, \ldots, y_{k}\right\} \cap Z \subseteq\left\{x_{\gamma}\right.$ : $\gamma<\alpha\}$ então se existe $V \in \mathcal{V}$ tal que $\emptyset \neq K\left(\left(y_{i}\right)_{i=1}^{k},\left(G_{j}\right)_{i=1}^{n},\left(B_{i}\right)_{i=1}^{l},\left(B_{j}^{\prime}\right)_{j=1}^{n}\right) \subseteq V$, então existe $\delta \in I \cap \alpha$ tal que $V_{\delta}$ satisfaz o que $V$ satisfaz.

Temos que $C$ é fechado e ilimitado. Vejamos que $C$ é fechado. Seja $\beta<\omega_{1}$ um ordinal limite tal que $C \cap \beta$ é ilimitado em $\beta$. Sejam $k, n \in \omega, y_{0}, \ldots, y_{k} \in \Sigma \cup Z, G_{0} \ldots, G_{n}$ abertos básicos de $(X, \mathcal{C}), B_{0}, \ldots, B_{k}, B_{0}^{\prime}, \ldots, B_{n}^{\prime} \in \mathcal{B}$. Suponha que $\left\{y_{0}, \ldots, y_{k}\right\} \cap Z \subseteq\left\{x_{\gamma}: \gamma<\beta\right\}$ e suponha que $V \in \mathcal{V}$ tal que $\emptyset \neq K\left(\left(y_{i}\right)_{i=1}^{k},\left(G_{j}\right)_{i=1}^{n},\left(B_{i}\right)_{i=1}^{l},\left(B_{j}^{\prime}\right)_{j=1}^{n}\right) \subseteq V$. Como $\left\{y_{0}, \ldots, y_{k}\right\}$ é finito, existe $\alpha$ com $\alpha \in C \cap \beta$ tal que $\left\{y_{0}, \ldots, y_{k}\right\} \cap Z \subseteq\left\{x_{\gamma}: \gamma<\alpha\right\}$, e então existe $\delta \in I \cap \alpha \subseteq I \cap \beta$, o que mostra que $\beta \in C$. Agora vejamos que $C$ é ilimitado. Dado $\beta<\omega_{1}$, existe $f(\beta) \in \omega_{1}$, com $f(\beta)>\beta$ tal que se para $k, n \in \omega, y_{0}, \ldots, y_{k} \in \Sigma \cup Z, G_{0} \ldots, G_{n}$ abertos básicos de $(X, \mathcal{C})$, $B_{0}, \ldots, B_{k}, B_{0}^{\prime}, \ldots, B_{n}^{\prime} \in \mathcal{B}$, se $\left\{y_{0}, \ldots, y_{k}\right\} \cap Z \subseteq\left\{x_{\gamma}: \gamma<\beta\right\}$ então se existe $V \in \mathcal{V}$ tal que $\emptyset \neq K\left(\left(y_{i}\right)_{i=1}^{k},\left(G_{j}\right)_{i=1}^{n},\left(B_{i}\right)_{i=1}^{l},\left(B_{j}^{\prime}\right)_{j=1}^{n}\right) \subseteq V$, então existe $\delta \in I \cap f(\beta)$ tal que $V_{\delta}$ satisfaz o que $V$ satisfaz. Dado $\beta<\omega_{1}$, temos que $\bigcup_{n \in \omega} f^{n}(\beta) \geq \beta$ está em $C$.

Fixemos $\alpha \in S \cap C$. Temos que $\mathcal{V}_{\alpha}$ é enumerável. Veremos que $\mathcal{V}_{\alpha}$ recobre o espaço. Suponha por absurdo que não, e tome $f \in C_{p}(\Sigma \cup Z) \backslash \cup \mathcal{V}_{\alpha}$. Seja $g=f \mid \Sigma$. Temos que $\operatorname{Osc}(g, \mathcal{T}) \cap Z=\emptyset$. Em particular, $\operatorname{Osc}(g, \mathcal{T}) \cap\left\{x_{\beta}: \beta<\alpha\right\}=\emptyset$.

Devemos ter, pela consequência da afirmação, que $\operatorname{Osc}(g, \mathcal{C})$ é magro. Além disso, $f$ é uma extensão $\mathcal{T}$-contínua de $g$ à $\Sigma \cup Z$. Podemos estender $f$ de forma contínua aos pontos de $2^{\omega} \backslash$ $\operatorname{Osc}(g, \mathcal{T})$, e como $f \notin \bigcup \mathcal{V}_{\alpha}$, temos que essa extensão também não está nesse conjunto.

Assim, no passo $\alpha$ da construção de $Z$, escolhemos uma função $g_{\alpha}$ tal que, por (i), pode ser $\mathcal{T}$-estendida continuamente para $\left\{x_{\beta}: \beta<\alpha\right\}$, e, pela escolha de $M_{\alpha}$ e dos $x_{\beta}$ para $\beta \leq \alpha$, temos que $g_{\alpha}$ pode ser estendida $\mathcal{C}$-continuamente para $\left\{x_{\beta}: \omega_{1}>\beta \geq \alpha\right\}$. Chamemos essa extensão de $h: \Sigma \cup Z \rightarrow \mathbb{R}$, que é $\mathcal{T}$-contínua.

Existe $V \in \mathcal{V}$ tal que $h \in V$. Escrevamos

$$
V=V\left(\left(x_{\alpha_{0}}, \ldots, x_{\alpha_{k}}, x_{\beta_{0}}, \ldots, x_{\beta_{n}}\right),\left(B_{0}, \ldots, B_{k}, B_{0}^{\prime}, \ldots, B_{n}^{\prime}\right)\right)
$$

onde cada $\alpha_{i}<\alpha, \beta_{i} \geq \alpha$.

Para cada $i \leq n$, seja $\epsilon_{i}>0$ tal que $\left\{t \in \mathbb{R}:\left|f\left(x_{\beta_{i}}\right)-t\right|<\epsilon_{i}\right\} \subseteq B_{i}^{\prime}$. Seja $\epsilon=\frac{1}{2} \min \left\{\epsilon_{0}, \ldots, \epsilon_{n}\right\}$. Temos que $\operatorname{Osc}(h, \epsilon, \mathcal{C}) \subseteq 2^{\omega}$ é fechado, magro e disjunto de $\left\{x_{\beta_{0}}, \ldots, x_{\beta_{n}}\right\}$. Então para cada $i \leq n$, existe uma $\mathcal{C}$-vizinhança $G_{i}$ de $x_{\beta_{i}}$ tal que $G_{i} \cap \operatorname{cl}_{\mathcal{C}} \operatorname{Osc}(h, \epsilon, \mathcal{C})=\emptyset$.

Se $x \in G_{i}$, temos que $\left|h(x)-h\left(x_{\beta_{i}}\right)\right| \leq \epsilon$, e, portanto, $h(x) \in B_{i}^{\prime}$. Logo:

$$
h \in K\left(\left(x_{\alpha_{i}}\right)_{i=1}^{k},\left(G_{i}\right)_{j=1}^{n},\left(B_{i}\right)_{i=1}^{k},\left(B_{j}^{\prime}\right)_{j=1}^{n}\right)
$$

Vale que:

$$
K\left(\left(x_{\alpha_{i}}\right)_{i=1}^{k},\left(G_{i}\right)_{j=1}^{n},\left(B_{i}\right)_{i=1}^{k},\left(B_{j}^{\prime}\right)_{j=1}^{n}\right) \subseteq V .
$$

Como $\alpha \in C$, temos que existe $\beta \in I \cap \alpha$ tal que: 


$$
h \in K\left(\left(x_{\alpha_{i}}\right)_{i=1}^{k},\left(G_{i}\right)_{j=1}^{n},\left(B_{i}\right)_{i=1}^{k},\left(B_{j}^{\prime}\right)_{j=1}^{n}\right) \subseteq V_{\beta}
$$

Porém, $h$ é uma (única) extensão $\mathcal{T}$-contínua de $g_{\alpha}, h \in V_{\beta} \in \mathcal{V}_{\alpha}$, o que contradiz a escolha de $g_{\alpha}$.

A. Dow e P. Simon utilizaram a proposição acima para responder parcialmente a seguinte pergunta, feita por Arhangel'skii: Suponha que $C_{p}(X \backslash\{x\})$ seja Lindelöf e que $x$ tenha tightness enumerável em $X . C_{p}(X)$ é Lindelöf?

Proposição 3.10. Assuma $\diamond$. Existe um espaço $X$ e $x \in X$ tal que $x$ possui tightness enumerável em $X, X \backslash\{x\}$ é primeiro-enumerável, $C_{p}(X \backslash\{x\})$ é Lindelöf mas $C_{p}(X)$ não é Lindelöf.

Demonstração. Seja $X$ a compactificação por um ponto do espaço $\Sigma \cup Z$ da proposição anterior, sendo $x$ o ponto que foi adicionado. Temos que $C_{p}(X \backslash\{x\})$ é Lindelöf, $X \backslash\{x\}$ é primeiro enumerável e $x$ possui tightness enumerável. Resta ver que $C_{p}(X)$ não é Lindelöf.

Para $n \in \omega$, seja $V_{n}=\left\{f \in C_{p}(X): \forall p \in 2^{n}(f(p) \in(-1,1))\right\}$. Para $z \in Z$, seja $V_{z}=\{f \in$ $\left.C_{p}(X): f(z) \neq 0\right\}$. Se $A \subseteq \Sigma, A=\{p, q\}$ e $p, q$ são funções incompatíveis, então $V_{A}=\left\{f \in C_{p}(X)\right.$ : $f(p) \neq 0, f(q) \neq 0\}$. Seja $\mathcal{V}$ a coleção de todos esses abertos.

Temos que $\mathcal{V}$ é uma cobertura: Fixe $f$. Se $f(x) \neq 0$, por continuidade existe $z \in Z$ tal que $f(z) \neq 0$, daí, $f \in V_{z}$. Se $f(x)=0$, então dividimos em casos novamente: Se existe $n$ tal que $f(p) \in(-1,1)$ para todo $p \in 2^{n}$, temos que $f \in V_{n}$. Caso contrário, temos novamente dois casos: Existem $p, q \in \Sigma$ incompatíveis distintas com $|f(p)| \geq 1 \leq|f(q)|$, e, nesse caso, $f \in V_{A}$, ou dado $n$ existe um único $p_{n} \in 2^{n}$ e $\left|f\left(p_{n}\right)\right| \geq 1$, sendo todos os $p_{n}$ compatíveis Nesse caso, sendo $g=\bigcup_{n \in \omega} p_{n} \in 2^{\omega}$. Temos que $g \in Z$, caso contrário teríamos que $x$ seria ponto de acumulação de $\left\{p_{n}: n \in \omega\right\}$, o que implicaria que $f(x) \neq 0$. Assim, temos que $f \in V_{g}$.

$\mathcal{V}$ não possui subcobertura enumerável, pois qualquer subcoleção enumerável de $\mathcal{V}$ não terá algum $V_{z}$ para algum $z \in Z$, e a função constante igual à 1 em $\{z \mid n: n \leq \omega\}$ e 0 no complementar é contínua e é elemento de qualquer membro desta subcobertura. 


\section{Capítulo 4}

\section{$\Psi$-espaços e espaços sequenciais}

\subsection{Introdução}

É bastante conhecido e amplamente utilizado o fato que em espaços métricos, sequências são utilizadas para determinar fechos de conjuntos. Iniciaremos essa seção motivando-a com a seguinte proposição, que na verdade é um exercício de muitos cursos básicos de Topologia Geral.

Proposição 4.1. Suponha que $X$ é um espaço topológico e $A \subseteq X$.

1. Se existe $s_{n}$ sequência em $A \operatorname{com} s_{n} \rightarrow x$ então $x \in \bar{A}$.

2. Se $x \in \AA$ então se $s_{n}$ é uma sequência em $X$ com $s_{n} \rightarrow x$ então $s_{n}$ está eventualmente em $A$.

Além disso, se $X$ é primeiro enumerável, então valem as recíprocas, e vale que:

i. $A$ é aberto se, e somente se, para todo $x \in A$, sempre que $s_{n}$ é uma sequência em $X$ com $s_{n} \rightarrow x$ então $s_{n}$ está eventualmente em $A$.

ii. $A$ é fechado se, e somente se, para toda sequência $s_{n}$ em $A$, o conjunto dos limites de $s_{n}$ está contido em $A$.

De certa forma, a proposição acima nos diz que se $X$ é primeiro enumerável, a topologia de $X$ é determinada pelas suas sequências. Explicitamente, se $\tau$ é a topologia de $X$, então $\tau=\{A \in$ $\mathscr{P}(X): \forall x \in A\left(\forall s \in{ }^{\omega} A\left(s_{n} \rightarrow x \rightarrow \exists n_{0} \in \omega\left(\forall n \geq n_{0}(n \in A)\right)\right)\right\}$.

Nessa seção, lidaremos com espaços sequenciais e de Fréchet. Como referências, citamos [Fra65] e [Fra67]. Outra referência para este assunto é [Sim03]. Ao final da seção, construiremos um exemplo clássico que utiliza espaços de Mrówka.

\subsection{Espaços sequenciais e espaços de Fréchet}

Nesta seção, definiremos o que são espaços sequenciais e de Fréchet e estudaremos algumas de suas propriedades básicas e exemplos. Começaremos definindo algumas coisas.

Definição 4.1. Seja $X$ um espaço topológico. Um subconjunto $U$ de $X$ é sequencialmente aberto em $X$ se, e somente se, toda sequência em $X$ que converge para algum ponto de $U$ está eventualmente em $U$. Um subconjunto $F$ de $X$ é sequencialmente fechado se, e somente se, sempre que $x_{n}$ é uma sequência em $F$ e $x_{n} \rightarrow x$ então $x \in F$.

Conjuntos sequencialmente abertos e sequencialmente fechados têm a mesma relação entre si que abertos e fechados. 
Lema 4.2. Seja $X$ um espaço topológico e $A \subseteq X$. Temos que $A$ é sequencialmente fechado se, e somente se, $X \backslash A$ for sequencialmente aberto.

Demonstração. Suponha que $A$ seja sequencialmente fechado. Se $s_{n} \rightarrow x \in X \backslash A$ não estiver eventualmente contida em $X \backslash A$, então ela possui uma subsequência contida em $A$ que converge para $x \in X \backslash A$, absurdo.

Agora suponha que $X \backslash A$ é sequencialmente aberto e seja $s_{n}$ uma sequência em $A \operatorname{com} s_{n} \rightarrow x$. Se $x \in X \backslash A$, temos que existe $n \in \omega$ com $s_{n} \in X \backslash A$, absurdo.

Estamos a um passo de definir espaços sequenciais.

Proposição 4.3. Considere $X$ um espaço topológico. As duas primeiras propriedades são equivalentes. Se $X$ é Hausdorff, as quatro propriedades são equivalentes:

1. Todo subconjunto sequencialmente aberto de $X$ é aberto.

2. Todo subconjunto sequencialmente fechado de $X$ é fechado.

3. Todo subconjunto de $X$ cuja interseção com toda sequência convergente é um conjunto fechado é fechado.

4. Todo subconjunto de $X$ cuja interseção com todo subespaço compacto metrizável de $X$ é um subconjunto fechado é fechado.

Demonstração. $1 \leftrightarrow 2$ : Suponha 2. Seja $F$ um subconjunto sequencialmente fechado de $X$. Pelo Lema 4.2, $X \backslash F$ é sequencialmente aberto, e, portanto, aberto. Assim, $F$ é fechado.

Suponha 2. Seja $A$ um subconjunto sequencialmente aberto de $X . X \backslash A$ é sequencialmente fechado, e, portando, fechado. Assim, $A$ é aberto.

Agora suponha que $X$ é Hausdorff.

$2 \leftrightarrow 3$ : Suponha 2. Seja $F$ um subconjunto de $X$ cuja interseção com toda sequência convergente é fechada. Veremos que $F$ é sequencialmente fechado, e, portanto, fechado. Seja $s_{n}$ uma sequência em $F \operatorname{com} s_{n} \rightarrow s_{\omega}$. Temos que $\left\{s_{n}: n \leq \omega\right\} \cap F$ é fechado e compacto. Se $s_{\omega} \notin F$, então $\left\{s_{n}: n<\omega\right\}$ é compacto. Mas $\left\{s_{n}: n<\omega\right\}$ é infinito e discreto, absurdo (se fosse finito, algum elemento de $s_{n}$ se repetiria infinitas vezes, daí o limite seria esse elemento. E o espaço é discreto pois dado $s_{n}$, se $A, B$ são abertos de $X$ disjuntos com $s_{n} \in A, s_{\omega} \in B$, temos que todos a menos de uma quantidade finita de elementos da sequência estão em $B$. Logo, $A \cap\left\{s_{m}: m<\omega\right\}$ é um aberto finito da sequência. Como conjuntos finitos são fechados, segue que $\left\{s_{n}\right\}$ é aberto na sequência).

Agora suponha 3. Tome $F$ sequencialmente fechado. Seja $s_{n}$ uma sequência em $X \operatorname{com} s_{n} \rightarrow s_{\omega}$. Temos que ver que $\left\{s_{n}: n \leq \omega\right\} \cap F$ é fechado. Se $\left\{s_{n}: n<\omega\right\} \cap F$ é finito, segue a tese. Caso contrário, devemos ter que $s_{\omega} \in F$, caso contrário, teremos uma subsequência de $s$ contida em $F$ que converge para fora de $F$. Então $\left\{s_{n}: n \leq \omega\right\} \backslash F \subseteq\left\{s_{n}: n<\omega\right\}$, que é aberto e discreto em $\left\{s_{n}: n \leq \omega\right\}$, e, assim, $\left\{s_{n}: n \leq \omega\right\} \backslash F$ é aberto em $\left\{s_{n}: n \leq \omega\right\}$. Logo, $\left\{s_{n}: n \leq \omega\right\} \cap F$ é compacto, e, portanto, fechado.

$3 \leftrightarrow 4$ : Supondo 3, 4 decorre diretamente pois toda sequência convergente é compacta e metrizável. Para ver isso, tome uma sequência que converge $s_{n}$. Ela é Hausdorff e enumerável (pela unicidade do limite), e portanto Lindelöf, e, assim, Tychonoff (ver [Wil04], 16.8). Além disso, a sequência convergente tem como base local para cada ponto, $\left\{\left\{s_{n}\right\}\right\}$, para os pontos da sequência, e, para o limite $x,\left\{\left\{s_{i}: i \geq n\right\}: n \in \omega\right\} \cup\{x\}$. É imediato que uma sequência convergente é compacta e que tem base enumerável, logo, também é metrizável (ver [Wil04], 23.1).

Agora suponha 4. Suponha que $F$ seja tal que a interseção com toda sequência convergente é fechada. Veremos que a interseção de $F$ com qualquer espaço metrizável compacto é fechado, o que implica que $F$ é fechado. Seja $K$ um subespaço métrico compacto. Veremos que $F \cap K$ é fechado. Como $K$ é compacto, basta ver que $F \cap K$ é fechado em $K$. Como $F \cap K$ é metrizável, este é primeiro 
enumerável, logo, basta ver que $F \cap K$ é sequencialmente fechado. Suponha que $s_{n}$ é uma sequência em $F \cap K$ que converge para $x \in K$. Temos que a interseção de $F$ com a sequência convergente é fechada, portanto compacta, e, como antes, isso implica que $x \in F$. Daí, $x \in F \cap K$.

A luz desta proposição, definiremos o que é um espaço sequencial e um espaço de Fréchet.

Definição 4.2. Seja $X$ um espaço topológico. Dizemos que $X$ é sequencial se satisfaz uma das (todas as) duas primeiras condições anteriores. Dizemos que um espaço topológico $X$ é de Fréchet se para todo $A \subseteq X$ e para todo $x \in \bar{A}$ existe uma sequência em $A$ que converge para $x$ (note que a recíproca é sempre verdadeira em qualquer espaço).

Proposição 4.4. Todo espaço de Fréchet é sequencial.

Demonstração. Se $X$ é um espaço de Fréchet, $A$ é sequencialmente fechado e $x \in \bar{A}$, existe uma sequência em $A$ que converge para $X$. Mas como $A$ é sequencialmente fechado, então $x \in A$. Logo, $\bar{A}=A$ e $A$ é fechado.

$\mathrm{Na}$ introdução apresentamos a seguinte propriedade:

Proposição 4.5. Todo espaço primeiro enumerável é de Fréchet, e, portanto, sequencial.

Um espaço sequencial é um espaço cuja topologia fica determinada por suas sequências. Todo espaço primeiro enumerável é sequencial, assim, todo espaço métrico e todo espaço discreto é sequencial. Porém, uma grande classe de espaços falha em ser sequencial ou Fréchet.

Proposição 4.6. Existem espaços compactos Hausdorff que não são sequenciais.

Daremos dois exemplos.

Demonstração. Considere $\omega_{1}+1$ com a topologia da ordem. A topologia da ordem é Hausdorff e ordinais sucessores são compactos. Porém, $\omega_{1}+1$ não é sequencial, pois $\left\{\omega_{1}\right\}$ é sequencialmente aberto: se $\alpha_{n} \rightarrow \omega_{1}$, então $\alpha_{n}$ deve ser eventualmente igual a $\omega_{1}$, caso contrário, podemos tomar uma subsequência $\beta_{n} \operatorname{com} \beta_{n} \rightarrow \omega_{1}$ mas $\beta_{n} \neq \omega_{1}$ para todo $n$. Temos que $\delta:=\sup \beta_{n}<\omega_{1}$, e a sequência não intersecta a vizinhança aberta $\left(\delta, \omega_{1}\right]$ de $\omega_{1}$.

Como um segundo exemplo apenas esboçado, $\beta \omega \backslash \omega$ é um espaço compacto totalmente desconexo de cardinalidade $2^{\mathfrak{c}}$ e sem pontos isolados em que todo subconjunto é sequencialmente fechado já que todo subconjunto fechado infinito dele tem cardinalidade $2^{\mathfrak{c}}$ ([Wal75], 3.12).

Almost disjoint families possuem uma propriedade que veremos ser essencial no estudo de espaços compactos de Fréchet com o que diz respeito à espaços de Mrówka.

Definição 4.3. Uma almost disjoint family $\mathcal{A}$ é maximal em lugar nenhum (nowhere mad) se para todo $X \in \mathcal{I}^{+}(\mathcal{A})$ existe $B \subseteq X$ com $|B|=\omega$ tal que $|B \cap A|<\omega$ para todo $A \in \mathcal{A}$.

Esse conceito se relaciona com $\Psi$-espaços da seguinte forma:

Proposição 4.7. Suponha que $\mathcal{A}$ é uma almost disjoint family. Então $Y=\Psi(\mathcal{A}) \cup\{\infty\}$ (a compactificação por um ponto de $\Psi(\mathcal{A}))$ é de Fréchet se, e somente se, $\mathcal{A}$ é nowhere MAD.

Demonstração. Suponha que $Y$ seja de Frechet. Tome $X \subseteq \omega$ tal que $X \in \mathcal{I}^{+}(\mathcal{A})$. Começaremos vendo que $\infty \in \bar{X}$. Para isso, devemos ver que se $K$ é compacto, $X \backslash Y \backslash K \neq \emptyset$. Fixe $K$ e considere a seguinte cobertura aberta de $Y$

$$
\{Y \backslash K\} \cup\{n: n \in \omega\} \cup\{\{A\} \cup A: A \in \mathcal{A}\}
$$

Por compacidade, existem $A_{1}, \cdots, A_{k}$ e $n$ tais que:

$$
Y=(Y \backslash K) \cup n \cup\left\{A_{1}, \cdots, A_{k}\right\} \cup\left(A_{1} \cup \cdots \cup A_{k}\right)
$$


Devemos ter que $X \cap Y \backslash K \neq \emptyset$, caso contrário, teríamos que $X \subseteq n \cup A_{1} \cup \cdots \cup A_{k}$.

Como $\infty \in \operatorname{cl} X$ e $Y$ é de Fréchet, existe uma sequência $s_{n} \rightarrow \infty$ de elementos de $X$ que pode ser tomada estritamente crescente. Seja $B$ a imagem da sequência. $B$ é infinito, $B \subseteq X$. Dado $A \in \mathcal{A}$, temos que $|A \cap B|<\omega$, caso contrário, a sequência possuiria uma subsequência que estaria eventualmente contida em qualquer aberto básico de $A$, o que implicaria que esta subsequência converge para $A$, o que viola a unicidade do limite ( $Y$ é Hausdorff).

Reciprocamente, suponha que $\mathcal{A}$ é nowhere MAD. Devemos ver que $Y$ é de Fréchet. Seja $Z \subseteq Y$ e suponha que $p \in \operatorname{cl} Z$. Suponhamos primeiro que $p \neq \infty$. Afirmo que $p \in \operatorname{cl}(Z \backslash\{\infty\})$, pois dado $A$ aberto básico contendo $p, A$ não tem $\infty$ como elemento e intersecta $Z$. Daí, como $\Psi(\mathcal{A})$ é de Fréchet, segue que existe uma sequência de elementos de $Z \backslash\{\infty\}$ que converge para $p$.

Agora suponha que $p=\infty$. Se $\infty \in Z$ tomamos a sequência constante. Suponha que $\infty \notin Z$. Temos que $\infty \in \operatorname{cl}(\omega \cap Z) \cup \operatorname{cl} Z \backslash \omega$.

Caso 1: $\infty \in \operatorname{cl}(\omega \cap Z)$. Seja $X=\omega \cap Z$. Afirmo que $X$ não é um subconjunto de uma união finita de elementos de $\mathcal{A} \cup[\omega]^{\omega}$, caso contrário, existiriam $n \in \omega$ e $A_{1}, \cdots, A_{k} \operatorname{com} X \subseteq\left\{A_{1}, \cdots, A_{k}\right\} \cup$ $A_{1} \cup \cdots \cup A_{k} \cup n$, que é compacto, e, portanto, $\infty \notin X$. Daí, existe $B \subseteq X$ infinito com $|B \cap A|<\omega$ para cada $A \in \mathcal{A}$. Escrevemos $B=\left\{x_{n}: n \in \omega\right\}$ em ordem crescente. Afirmo que $x_{n} \rightarrow \infty$, pois, se $K$ é compacto, existem $A_{1}, \cdots, A_{k} \in \mathcal{A}$ e $m \in \omega \operatorname{com} Y=(Y \backslash K) \cup\left\{A_{1}, \cdots, A_{k}\right\} \cup A_{1} \cup \cdots \cup A_{k} \cup m$. Temos que $\left|B \cap A_{j}\right|<\omega$ para cada $j$, assim, há uma quantidade cofinita de elementos de $B$ em $Y$.

Caso 2: $\infty \in \operatorname{cl}(Z \backslash \omega)$. Temos que $Z \backslash \omega \subseteq \mathcal{A}$ é infinito. Tomemos uma sequência injetora em $Z \backslash \omega, A_{n}(n \in \omega)$. Afirmo que $A_{n} \rightarrow \infty$. Para ver isso, se $K \subseteq \Psi(\mathcal{A})$ é compacto, $K$ possui apenas uma quantidade finita de elementos de $\mathcal{A}$, pois a base $\mathcal{B}$ é uma cobertura aberta de $K$. Dessa forma, $A_{n}$ está eventualmente contida no complemento de $K$.

Corolário 4.8. Se $\mathcal{A}$ é uma MAD family, então, sendo $Y$ a compactificação por um ponto de $\Psi(\mathcal{A})$, $Y$ é um espaço compacto, Hausdorff e sequencial que não é de Fréchet.

Demonstração. Seja $\mathcal{A}$ uma MAD family infinita de $\omega$ e considere $Y$. Temos que $Y$ é compacto Hausdorff. $X$ não é de Fréchet pois $\mathcal{A}$ não é nowhere MAD. Para ver isso, primeiro notemos que $\omega \notin \mathcal{I}^{+}(\mathcal{A})$, então, se $\mathcal{A}$ fosse nowhere $\mathrm{MAD}$, existiria um subconjunto infinito de $\omega$ que tem interseção finita com todo elemento de $\mathcal{A}$, o que viola a maximalidade da família.

Resta ver que $Y$ é sequencial. Suponha que $U \subseteq Y$ é sequencialmente aberto. Veremos que $U$ é vizinhança de todos os seus pontos, e, portanto, aberto. $U$ é vizinhança de todos os seus números naturais. Se $A \in \mathcal{A} \cap U$, note que enumerando $A$ em ordem crescente pela sequência $s_{n}$, temos que $s_{n} \rightarrow A$, daí, $s_{n}$ está eventualmente contida em $U$, e com isso temos uma vizinhança de $A$ contida em $U$. Se $\infty \in U$, veremos que $K=Y \backslash U$ é compacto. Temos que $K \cap \mathcal{A}=\left\{A_{1}, \cdots, A_{k}\right\}$ é finito, caso contrário teríamos uma sequência disjunta de $U$ que converge para $\infty$. Além disso, se $A \in \mathcal{A} \cap U$, então $A \cap K$ é finito, pois como vimos, $A$ possui uma vizinhança em $U$. Assim, se $\mathscr{U}$ é uma cobertura aberta de $K$, ela deve possuir abertos $U_{1}, \cdots, U_{k}$ que cobrem $A_{1}, \cdots, A_{k}$. Seja $V=U_{1} \cup \cdots \cup U_{k}$. Basta ver que $K \backslash V$ é finito. Suponha que não seja. Então $B=\omega \cap K \backslash V$ é infinito. Por construção, temos que $\left|B \cap A_{i}\right|<\omega$ para cada $i \leq k$. Se $A \in U$, então $A$ só tem uma quantidade finita de pontos em $K, \operatorname{logo},|B \cap A|<\omega$. Mas isso viola que $\mathcal{A}$ é maximal.

Construiremos espaços topológicos compactos Hausdorff $X$ e $Y$ de Fréchet cujo produto não é de Fréchet. Para isso, precisaremos das seguintee proposição:

Proposição 4.9. Seja $\mathcal{A}$ uma almost disjoint family e $X \in \mathcal{I}^{+}(\mathcal{A})$ tal que todo subconjunto infinito de $X$ intersecta um elemento de $\mathcal{A}$. Define-se $\mathcal{A} \mid X=\{A \cap X: A \in \mathcal{A}\} \backslash[\omega]^{<\omega}$. Este conjunto é uma MAD family em $X$.

Demonstração. Vejamos que $\mathcal{A} \mid X$ é infinito. Suponha por absurdo que seja finito. Então $\{B \in \mathcal{A}$ : $|B \cap X|=\omega\}$ é finito. Enumere-o por $\left\{B_{0}, \ldots, B_{n}\right\}$. Temos que $A \backslash \bigcup_{i \leq n} B_{i}$ é infinito, logo, este intersecta algum elemento $B$ de $\mathcal{A}$ infinitamente. Note que $B \neq B_{i}$ para todo $i$ e $B \cap\left(B_{0} \cup \ldots B_{n}\right)$ é infinito, absurdo.

Para a maximalidade, note que dado $Y \subseteq X$ infinito, temos que existe $A \in \mathcal{A}$ tal que $|Y \cap A|=\omega$. Logo, $X \cap A \in \mathcal{A} \mid X$ e $|Y \cap(X \cap A)|=|Y \cap A|=\omega$. 
Proposição 4.10. Existe uma MAD family $\mathcal{A}$ que pode ser particionada em duas nowhere MAD almost disjoint families.

Demonstração. Suponha por absurdo que não exista nenhuma MAD family de $\omega$ que pode ser particionada desta forma. Então não existe nenhuma MAD family de nenhum subconjunto infinito enumerável que pode ser particionada desta forma, caso contrário, via bijeção, obteríamos uma de $\omega$ que pode.

Seja $\mathcal{A}$ uma MAD family de cardinalidade $\mathfrak{c}$ e indexemos-a escrevendo $\mathcal{A}=\left\{A_{f}: f \in{ }^{\omega_{2}} 2\right\}$. Para cada $n \in \omega$, seja $P_{n}^{0}=\left\{A_{f}: f(n)=0\right\}, p_{n}^{1}=\left\{A_{f}: f(n)=1\right\}$. Note que para cada $n,\left\{P_{n}^{0}, P_{n}^{1}\right\}$ particiona $\mathcal{A}$ e $\left|P_{n}^{0}\right|=\left|P_{n}^{1}\right|=2^{\omega}$.

Definiremos recursivamente uma sequência decrescente em $I^{+}(A) X_{n}$ e também $g: \omega \rightarrow 2$, de modo que $P_{n}^{g(n)} \mid X_{n}$ seja uma MAD family em $X_{n}$.

Pela hipótese de absurdo, existe $g(0)<2$ tal que $P_{0}^{g(0)}$ não é nowhere MAD. Tome $X_{0} \in$ $\mathcal{I}^{+}\left(\mathcal{P}_{0}^{g(0)}\right)$ tal que todo subconjunto de $X_{0}$ infinito possui interseção infinita com algum elemento de $P_{0}^{g(0)}$. Pela proposição anterior, $P_{0}^{g(0)} \mid X_{0}$ é uma MAD family.

Afirmo que $X_{0} \in I^{+}(\mathcal{A})$. Suponha por absurdo que não. Então existem $A_{0}, \ldots, A_{n} \in P_{0}^{g(0)} \mathrm{e}$ $B_{0}, \ldots B_{m} \in P_{0}^{1-g(0)}$ tais que $X_{0} \subseteq^{*} A_{0} \cup \cdots \cup A_{n} \cup B_{0} \cup \cdots \cup B_{m}$. Assim, $X_{0} \backslash\left(A_{0} \cup \cdots \cup A_{n}\right) \subseteq^{*}$ $B_{0} \cup \cdots \cup B_{m}$, e $X_{0} \backslash\left(A_{0} \cup \cdots \cup A_{n}\right)$ é infinito pois $X_{0} \in I^{+}\left(P_{0}^{g(0)}\right)$. Assim existe $Y \in P_{0}^{g(0)}$ tal que $Y \cap\left(B_{0} \cup \cdots \cup B_{m}\right)$ é infinito, o que é absurdo.

Suponha que foi definido $X_{n}$ e $g(n)$. Temos que $\mathcal{A}\left|X_{n}=\mathcal{P}_{n+1}^{0}\right| X_{n} \cup P_{n+1}^{1} \mid X_{n}$. Ambos $P_{n+1}^{0} \mid X_{n}$ e $P_{n+1}^{1} \mid X_{n}$ forem infinitos, um deles é não é nowhere MAD, e se um deles for finito, o outro não é nowhere $\mathrm{MAD}$ (O que se conclui removendo de $X_{n}$ a união do outro). Seja $g(n+1)$ tal que $\mathcal{P}_{n+1}^{g(n+1)} \mid X_{n}$ não é nowhere MAD e fixe $X_{n+1} \subseteq X_{n}$ que atesta isso, ou seja, fora do ideal livre em $X_{n}$ gerado por $\mathcal{P}_{n+1}^{g(n+1)} \mid X_{n}$ e tal que qualquer subconjunto infinito de $X_{n+1}$ intersecta infinitamente um elemento de $P_{n+1}^{g(n+1)} \mid X_{n}$. Pela proposição anterior, $\mathcal{P}_{n+1}^{g(n+1)} \mid X_{n+1}$ é MAD family. Resta apenas ver que $X_{n+1} \in \mathcal{I}^{+}(\mathcal{A})$. Suponha por absurdo que não. Então existem $A_{0}, \ldots, A_{k} \in P_{0}^{g(n+1)}$ e $B_{0}, \ldots, B_{m} \in P_{0}^{1-g(n+1)}$ tais que $X_{n+1} \subseteq^{*} \bigcup_{i \leq k} A_{i} \cup \bigcup_{i \leq m} B_{i}$. Daí, $X_{n+1} \backslash \bigcup_{i \leq k}\left(A_{i} \cap X_{n}\right) \subseteq^{*}$ $\bigcup_{i \leq m}\left(B_{i} \cap X_{n}\right)$ e $X_{n+1} \backslash \bigcup_{i<k}\left(A_{i} \cap X_{n}\right)$ é infinito, o que implica novamente em um absurdo.

Pela Proposição 2.50, existe $Y$ em $\mathcal{I}^{+}(\mathcal{A})$ pseudointerseção $\operatorname{dos} X_{n}$. Como $Y \in \mathcal{I}^{+}(\mathcal{A})$, existem infinitos $f \in 2^{\omega} \operatorname{com}\left|A_{f} \cap Y\right|=\omega$.

Sabemos que existe $f \neq g$ tal que $Y \cap A_{f}$ é infinito. Tome $n$ com $f(n) \neq g(n)$. Temos que $A_{g}$ e $A_{f}$ estão em partições distintas de $\left\{P_{n}^{0}, P_{n}^{1}\right\}$. Se $g(n)=i, P_{n}^{i} \mid X_{n}$ é uma MAD family em $X_{n}$. Como $f(n)=1-i, A_{f} \notin P_{n}^{i}$, logo $\left|A_{f} \cap A\right|<\omega$ para todo $A \in P_{n}^{i}$. Mas então $A_{f} \cap X_{n}$ é finito, ou isso violaria a maximalidade de $P_{n}^{i} \mid X_{n}$. Mas então $\left(Y \cap A_{f}\right) \backslash\left(A_{f} \cap X_{n}\right)$ é infinito. Mas então $Y \backslash X_{n}$ é infinito, o que contradiz $Y \subseteq^{*} X_{n}$.

Proposição 4.11. Existem dois espaços compactos Hausdorff separáveis de Fréchet cujo produto não é de Fréchet.

Demonstração. Pela Proposição 4.10, seja $\mathcal{A}$ uma MAD family que pode ser particionada em duas nowhere MAD almost disjoint families, $\mathcal{A}_{1}$ e $\mathcal{A}_{2}$. Suas respectivas compactificações por um ponto, $Y_{1}$ e $Y_{2}$, são espaços separáveis, compactos, Hausdorff e de Fréchet. Em $Y_{1} \times Y_{2}$, considere o conjunto $A=\{(n, n): n \in \omega\}$. Afirmo que $p:=(\infty, \infty) \in \bar{A}$. Verifiquemos isso. Um aberto básico de $p$ é do tipo $\left(Y_{1} \backslash K_{1}\right) \times\left(Y_{2} \backslash K_{2}\right)$, com $K_{1}, K_{2}$ compactos de $\Psi\left(\mathcal{A}_{1}\right), \Psi\left(\mathcal{A}_{2}\right)$, respectivamente. Por compacidade, existem $A_{1}, \cdots, A_{k} \in \mathcal{A}_{1}, B_{1}, \cdots, B_{l} \in \mathcal{A}_{2}$ e $m, n \in \omega$ tal que $Y_{1}=\left(Y_{1} \backslash K_{1}\right) \cup A_{1} \cup$ $\cdots \cup A_{k} \cup m$ e $Y_{2}=\left(Y_{2} \backslash K_{2}\right) \cup B_{1} \cup \cdots \cup B_{l} \cup n$ Se $B \in \mathcal{A}$ é um elemento de $\mathcal{A}$ não listado, ele tem interseção finita com cada $A_{i}, B_{j}, m, n$, então, existe um ponto $b$ de $B$ fora de todos esses ( $B$ é infinito). Segue que $(b, b) \in\left(Y_{1} \backslash K_{1}\right) \times\left(Y_{2} \backslash K_{2}\right)$. Dessa forma, $(\infty, \infty) \in \bar{A}$. 
Suponha por absurdo que existe uma sequência $\left(s_{n}, s_{n}\right)$ de elementos de $A$ que converge para $p$. Sem perda de generalidade, podemos considerar que $s_{n}$ é uma sequência injetora. Seja $B=\left\{s_{n}\right.$ : $n \in \omega\}$. Temos que $B$ é um subconjunto infinito de $\omega$. Afirmo que $|C \cap B|<\omega$ para cada $C \in \mathcal{A}$, o que é absurdo. Sem perda de generalidade, $C \in \mathcal{A}_{1}$. Como $s_{n} \rightarrow \infty$ em $Y_{1}$ e $\{C\} \cup C$ é compacto, todos os elementos de $B$ a menos de uma quantidade finita estão no complemento de $\{C\} \cup C$. Daí, há apenas uma quantidade finita de elementos de $B$ em $C$.

Isso encerra o nosso exemplo. Uma pergunta pertinente é se o espaço acima é sequencial. Veremos que sim. Para isso, provaremos agora uma condição suficiente para que o produto de dois espaços sequenciais seja sequencial.

Lema 4.12. Suponha que $X, C$ são espaços sequenciais e $C$ é sequencialmente compacto. Então se $F \subseteq X$ é sequencialmente fechado, temos que $\pi_{X}[F]$ é fechado.

Demonstração. Suponha que $\pi_{X}[F]$ não seja fechado. Como $X$ é sequencial, existe uma sequência $x_{n}$ em $\pi_{X}[F]$ e $x \in X \backslash F \operatorname{com} x_{n} \rightarrow x$. Para cada $n$, existe $y_{n} \in C$ com $\left\langle x_{n}, y_{n}\right\rangle \in F$. Existe uma subsequência de $y_{n}$ e $y \in C \operatorname{com} y_{n_{k}} \rightarrow y$. Mas então $\left\langle x_{n_{k}}, y_{n_{k}}\right\rangle \rightarrow\langle x, y\rangle \notin F$, e esta é uma sequência em $F$, o que viola que $F$ é sequencialmente fechado.

Proposição 4.13. Suponha que $X, Y$ são espaços sequenciais e $Y$ tem um sistema fundamental de vizinhanças fechadas sequencialmente compactas e $X$ é T1, então $X \times Y$ é sequencial.

Demonstração. Suponha por absurdo que $X \times Y$ não seja sequencial. Então existe um conjunto sequencialmente fechado $A \subseteq X \times Y$ e $p \in \bar{A} \backslash A$ tal que não existe uma sequência em $A$ que converge para $p$. Seja $p=\langle x, y\rangle$.

Seja $B=(\{x\} \times Y) \cap A$. Afirmo que $B$ é sequencialmente fechado. Para ver isso, note que $B=\{x\} \times\{y \in Y:\langle x, y\rangle \in A\}$. Temos que $\{x\}$ é fechado. Basta ver que o segundo conjunto também é fechado. Para ver isso, basta ver que ele é sequencialmente fechado. Ora, se $y_{n}$ é uma sequência neste conjunto que converge para $z,\left\langle x, y_{n}\right\rangle$ é uma sequência em $A$ que converge para $\langle x, z\rangle$. Logo, $\langle x, z\rangle \in A$, e, assim, $z$ está no segundo conjunto. Isso mostra que ele é sequencialmente fechado e portanto fechado.

Dessa forma, $B$ é fechado. Como $p \notin A$, temos que existe $U$ vizinhança aberta de $p$ disjunta de $B$. Seja $C$ uma vizinhança fechada sequencialmente compacta de $y$ com $p \in\{x\} \times C \subseteq U$. Seja $A_{0}=(X \times C) \cap A$. Temos que $A_{0}$ é sequencialmente fechado. $p \in \bar{A}_{0} \backslash A_{0}$ pois $p \notin A$ mas se $V \times W$ é uma vizinhança aberta básica de $p$, temos que $V \times(W \cap \operatorname{int} C)$ também é, e esta deve intersectar $A$ e portanto $A_{0}$.

Seja $P=\pi_{X}\left[A_{0}\right]$. Pelo lema anterior aplicado à $X \times C, P$ é fechado em $X$. Então $(X \backslash P) \times Y$ é aberto. Note que $p \in(X \backslash P) \times Y$, pois $x \notin P$, caso contrário existiria $z \in Y \operatorname{com}\langle x, z\rangle \in A_{0}$, mas daí $\langle x, z\rangle \in U \cap B$, absurdo. Além disso, $(X \backslash P) \times Y$ é disjunto de $A_{0}$, o que viola que $p \in \bar{A}_{0}$. 


\section{Capítulo 5}

\section{Os espaços $\beta(\Psi(\mathcal{A}))$}

No capítulo anterior, utilizamos a compactificação por um ponto de um espaço de Mrówka. Agora, veremos alguns teoremas que dizem respeito a propriedades de outras compactificações de espaços de Mrówka, dando maior enfoque para a compactificação de Stone-Čech.

Veremos quando que um $\Psi$-espaço admite uma compactificação por dois pontos, ou, equivalentemente, quando o seu remainder de sua compactificação de Stone-Čech é desconexo. A seguir, estudaremos o problema sobre quando que um $\Psi$-espaço possui uma única compactificação (sendo esta a compactificação por um ponto), e, por fim, veremos que todo espaço métrico compacto sem pontos isolados é um remainder de uma compactificação de Stone-Čech de um $\Psi$-espaço de uma MAD family de cardinalidade $\mathfrak{c}$.

\subsection{Compactificação por dois pontos}

Nessa seção, veremos uma condição para que $\Psi(\mathcal{A})$ possua uma compactificação por dois pontos. Para isso, introduziremos a noção de particionador.

Definição 5.1. Seja $\mathcal{A}$ uma almost disjoint family. Dizemos que $X \subseteq \omega$ é um particionador de $\mathcal{A}$ se para todo $A \in \mathcal{A}$, temos que $A \subseteq^{*} X$ ou $A \subseteq^{*} \omega \backslash X$.

Dizemos que um particionador $X$ de $\mathcal{A}$ é um particionador não trivial se, e somente se, $X \in$ $\mathcal{I}^{+}(\mathcal{A})$ e $\omega \backslash X \in \mathcal{I}^{+}(\mathcal{A})$.

Notemos que se $X$ é um particionador (não trivial) para $\mathcal{A}$, então $X \backslash A$ também é. Notemos também que não podem ocorrer ambos $A \subseteq^{*} X$ e $A \subseteq^{*} X \backslash A$ ao mesmo tempo, caso contrário teríamos que $A \subseteq^{*} X \cap(\omega \backslash X)=\emptyset$, o que implica em $|A|<\omega$, o que é absurdo.

Proposição 5.1. Seja $\mathcal{A}$ uma almost disjoint family e suponha que $X$ é um particionador de $\mathcal{A}$. Sejam $p, q$ distintos com $p, q \notin \Psi(\mathcal{A})$. Seja $\tau$ a topologia de $\Psi(\mathcal{A})$. Sejam:

$$
\begin{gathered}
\mathcal{A}_{1}=\left\{A \in \mathcal{A}: A \subseteq^{*} X\right\} \\
\mathcal{A}_{2}=\left\{A \in \mathcal{A}: A \subseteq^{*} \omega \backslash X\right\}
\end{gathered}
$$

Então, denotando por $Y=\omega \backslash X$, o seguinte conjunto é uma base para uma topologia em $K=\Psi(\mathcal{A}) \cup\{p, q\}:$

$$
\begin{aligned}
\tau^{\prime} & =\tau \cup\left\{\{p\} \cup\left(\tilde{X} \backslash \bigcup_{i<n} A_{i}\right) \cup\left(\mathcal{A}_{1} \backslash\left\{A_{i}\right\}_{(i<n)}\right):\left(A_{i}\right)_{(i<n)} \text { é seq. finita em } \mathcal{A}_{1} \text { e } \tilde{X} \subseteq X \text { é cofinito }\right\} \\
& \cup\left\{\{q\} \cup\left(\tilde{Y} \backslash \bigcup_{i<n} A_{i}\right) \cup\left(\mathcal{A}_{2} \backslash\left\{A_{i}\right\}_{(i<n)}\right):\left(A_{i}\right)_{(i<n)} \text { é seq. finita em } \mathcal{A}_{2} \text { e } \tilde{Y} \subseteq Y \text { é cofinito }\right\}
\end{aligned}
$$

Na notação anterior, permite-se que sequências finitas sejam vazias. 
Nessa topologia, $K$ é compacto Hausdorff. Além disso, $\Psi(\mathcal{A})$ é denso em $K$ se, e somente se, $X$ for um particionador não trivial.

Demonstração. Primeiro, vejamos que $\tau^{\prime}$ é base para uma topologia em $K$. Está claro que $\bigcup \tau^{\prime}=K$. Dados dois elementos de $\tau$, a interseção deles está em $\tau$. Também está claro que a interseção de dois elementos do segundo conjunto sendo unido na definição de $\tau^{\prime}$ também é um elemento do segundo conjunto, e o mesmo vale para o terceiro conjunto. A interseção de um conjunto do segundo tipo com um conjunto do terceiro tipo é um subconjunto de $\omega$, portanto, elemento de $\tau$.

Agora suponha que $U \in \tau$ e $\left(A_{i}\right)_{(i<n)}$ é uma sequência finita de elementos de $\mathcal{A}_{1}$ e $\tilde{X} \subseteq X$ é cofinito. Suponha que $x \in U \cap\left(\{p\} \cup\left(\tilde{X} \backslash \bigcup_{i<n} A_{i}\right) \cup\left(\mathcal{A}_{1} \backslash\left\{A_{i}\right\}_{(i<n)}\right)\right)$. Como $x \in U$, temos que $x \neq p$. Se $x \in \omega$, temos que $\{x\}$ é aberto e é subconjunto dessa interseção. Se $x \in \mathcal{A}$, temos que $x \in \mathcal{A}_{1}$ e $x=B \notin\left\{A_{i}\right\}_{(i<n)}$. Como $B \in \mathcal{A}_{1}$, temos que $B \subseteq^{*} \tilde{Y}$. Seja $n$ tal que $B \backslash n \subseteq \tilde{X}$ e tal que $B \cap\left(\bigcup_{i<n} A_{i}\right) \subseteq n$. Segue que $\{B\} \cup(B \backslash n) \in \tau$ está contido na interseção.

Analogamente, prova-se que para todo $U \in \tau$, para toda $\left(A_{i}\right)_{(i<n)}$ sequência finita de elementos de $\mathcal{A}_{2}$, para todo $\tilde{Y} \subseteq Y$ cofinito e para todo $x \in U \cap\left(\{q\} \cup\left(\tilde{Y} \backslash \bigcup_{i<n} A_{i}\right) \cup\left(\mathcal{A}_{2} \backslash\left\{A_{i}\right\}_{(i<n)}\right)\right)$, existe $V \in \tau$ subconjunto dessa interseção com $x \in V$.

Logo, $\tau^{\prime}$ é uma base para uma topologia em $K$. Temos que $K$ é um espaço de Hausdorff: quaisquer dois pontos de $\Psi(\mathcal{A})$ são separados por elementos de $\tau$. Quaisquer dois abertos básicos de $p, q$ são disjuntos, portanto os separam. Se $n$ é natural, temos que $\{n\},\{p\} \cup(X \backslash\{n\}) \cup \mathcal{A}_{1}$ e $\{q\} \cup(Y \backslash\{n\}) \cup \mathcal{A}_{2}$ são abertos básicos dois-a-dois disjuntos. Se $A \in \mathcal{A}_{1}$, temos que $\{A\} \cup A$, $\{p\} \cup(X \backslash A) \cup\left(\mathcal{A}_{1} \backslash\{A\}\right)$ e $\{q\} \cup Y \cup \mathcal{A}_{2}$ são abertos disjuntos. Finalmente, se $A \in \mathcal{A}_{2}$, temos que $\{A\} \cup A,\{q\} \cup(Y \backslash A) \cup\left(\mathcal{A}_{2} \backslash\{A\}\right)$ e $\{p\} \cup X \cup \mathcal{A}_{1}$ são abertos disjuntos.

Temos que o espaço é compacto: seja $\mathcal{U}$ uma cobertura de $K$ por abertos básicos. Temos que existem $A_{p} \in \mathcal{U}$ com $p \in A_{p}$, e $A_{q} \in \mathcal{U}$ com $q \in A_{q}$. Assim, existem $\tilde{X} \subseteq X$ cofinito, $\tilde{Y} \subseteq Y$ cofinito, $\left(A_{i}\right)_{(i<n)}$ sequência em $\mathcal{A}_{1},\left(B_{j}\right)_{(i<m)}$ sequência em $\mathcal{A}_{2}$ com:

$$
\begin{aligned}
& A_{p}=\{p\} \cup\left(\tilde{X} \backslash \bigcup_{i<n} A_{i}\right) \cup\left(\mathcal{A}_{1} \backslash\left\{A_{i}\right\}_{(i<n)}\right) \in \mathcal{U} \\
& A_{q}=\{q\} \cup\left(\tilde{Y} \backslash \bigcup_{i<m} B_{i}\right) \cup\left(\mathcal{A}_{2} \backslash\left\{b_{i}\right\}_{(i<m)}\right) \in \mathcal{U}
\end{aligned}
$$

Dado $i<n$, existe $U_{i} \in \mathcal{U}$ com $A_{i} \in U_{i}$, logo, existe $n_{i} \in \omega$ com $\left\{A_{i}\right\} \cup\left(A_{i} \backslash n_{i}\right) \subseteq U_{i}$. Dado $j<m$, existe $V_{j} \in \mathcal{U}$ com $B_{j} \in V_{j}$, logo, existe $m_{j} \in \omega \operatorname{com}\left\{B_{j}\right\} \cup\left(B_{j} \backslash m_{j}\right) \subseteq V_{j}$. Sendo $k \in \omega$ tal que $k>n_{i}$ para todo $i<n, k>m_{j}$ para todo $j<m$ e tal que $X \subseteq \tilde{X} \cup k, Y \subseteq \tilde{Y} \cup k$, temos que $K \backslash k \subseteq A_{p} \cup A_{q} \cup \bigcup_{i<n} U_{i} \cup \bigcup_{j<m} V_{j}$. Como $k$ é finito, segue a tese.

Para a última afirmação, no caso de $X$ ser um particionador não trivial, precisamos ver que $\{p\}$ não é um aberto básico, nem $\{q\}$ um aberto básico. Para ver que $\{p\}$ não é um aberto básico, notemos que, se for, então existem $A_{1}, \ldots, A_{n} \in \mathcal{A} \operatorname{com} X \subseteq^{*} A_{1} \cup \cdots \cup A_{n}$, o que viola o fato de que $X \in \mathcal{I}^{+}(\mathcal{A})$. Analogamente, $\{q\}$ não é aberto básico. Reciprocamente, se $\Psi(\mathcal{A})$ for denso, então $\omega$ é denso (pois $\omega$ é denso em $\Psi(\mathcal{A})$ ). Logo, dados $A_{0}, \ldots, A_{n-1} \in \mathcal{A}_{1}$, temos que $\left|X \backslash \bigcup_{i<n} A_{i}\right|=\omega$. Além disso, dados $B_{0}, \ldots, B_{m-1} \in \mathcal{A}_{2}$, temos que $\left|X \cap \bigcup_{j<m} B_{m}\right|<\omega, \operatorname{logo},\left|X \backslash \bigcup_{j<m} B_{j} \backslash \bigcup_{i<m} A_{i}\right|=\omega$. Isso mostra que $X \in \mathcal{I}^{+}(\mathcal{A})$. Analogamente, $Y \in \mathcal{I}^{+}(\mathcal{A})$.

Provaremos agora um fato geral sobre o remainder da compaciticação de Stone-Čech de um espaço topológico genérico.

Lema 5.2. Sejam $n>1$ natural e $X$ um espaço topológico de Tychonoff. Então se $X$ admite uma compactificação por $n$ pontos, temos que $X^{*}$ é desconexo.

Demonstração. Seja $K=X \cup\left\{x_{1}, \ldots, x_{n}\right\}$ uma compactificação por $n$ pontos de $X$, e tome $f$ : $X \rightarrow K$ a inclusão, que é contínua. Tome $F: \beta X \rightarrow K$ a extensão contínua de $f$. Temos por 1.35 
que $F[\beta X \backslash e[X]]=\left\{x_{1}, \ldots, x_{n}\right\}$, logo $F^{-1}\left[\left\{x_{1}\right\}\right] \backslash X, \ldots, F^{-1}\left[\left\{x_{n}\right\}\right] \backslash X$ é uma partição de $\beta X \backslash X$ por $n$ clopens de $X^{*}$ disjuntos.

Juntando as proposições anteriores, obtemos o corolário:

Corolário 5.3. Se $\mathcal{A}$ possui um particionador não trivial, então $\Psi(\mathcal{A})^{*}$ não é conexo.

Finalmente, sintetizamos o nosso teorema. Uma versão deste resultado pode ser encontrada no artigo [I.B80].

Teorema 5.4. Seja $\mathcal{A}$ uma almost disjoint family. São equivalentes:

(a) $\mathcal{A}$ admite um particionador não trivial.

(b) $\Psi(\mathcal{A})$ admite uma compactificação por 2 pontos.

(c) $\Psi(\mathcal{A})^{*}$ não é conexo.

Demonstração. Resta apenas mostrar que $(c) \rightarrow(a)$. Suponha que $\Psi(\mathcal{A})^{*}$ não é conexo e fixe $C$ clopen não vazio de $\Psi(\mathcal{A})^{*}$ tal que $\Psi(\mathcal{A})^{*} \backslash C$ seja não vazio. Por 1.53 , temos que $\Psi(\mathcal{A})^{*}$ é fechado em $\beta(\Psi(\mathcal{A}))$. Assim, ambos $C$ e $\Psi(\mathcal{A})^{*} \backslash C$ são fechados em $\beta(\Psi(\mathcal{A}))$, e esses são disjuntos. Então, por normalidade existem $U, V$ abertos disjuntos de $\beta(\Psi(\mathcal{A}))$ com $C \subseteq U, \Psi(\mathcal{A})^{*} \backslash C \subseteq V$.

Para os parágrafos seguintes, identificaremos $e[\Psi(\mathcal{A})] \operatorname{com} \Psi(\mathcal{A})$.

Seja $F=e[\mathcal{A}] \backslash(U \cup V)$. Temos que $F$ é finito, caso contrário teria um ponto de acumulação $x$. Como $\mathcal{A}$ não tem ponto de acumulação em $\Psi(\mathcal{A})$, temos que $x \in U \cup V$. Mas $U \cup V$ é um aberto que não intersecta $F$, absurdo.

Seja $P=(U \cap \omega) \backslash \bigcup F$. Afirmo que $P$ é um particionador não trivial: dado $A \in \mathcal{A}$, temos que $A \in U$, ou $A \in V$, ou $A \in F$, e apenas uma dessas três possibilidades vale. Se $A \in U$, temos que, como $U$ é aberto, $A \subseteq^{*} U \cap \omega$, e como $A \notin F$ e $\mathcal{A}$ é almost disjoint, segue que $A \in P$. Se $A \in V$, temos que $A \subseteq^{*} V \cap \omega \subseteq \omega \backslash P$, e se $A \in F$, temos que $A \subseteq \omega \backslash P$. Resta ver que $P$ é não trivial. Para ver que $P \in \mathcal{I}^{+}(\mathcal{A})$, basta ver que $U \cap \omega \in I^{+}(\mathcal{A})$, pois $F$ é finito. Suponha por absurdo que existem $A_{1}, \ldots, A_{n} \in \mathcal{A}$ e $k \in \omega$ com

$$
U \cap \omega \subseteq A_{1} \cup \cdots \cup A_{n} \cup k
$$

Se $B \in U \cap \mathcal{A}$ e $B \notin\left\{A_{1}, \ldots, A_{n}\right\}$, temos que intersectando a expressão acima com $B$, obtemos um conjunto infinito contido em um finito. Logo, $U \cap \mathcal{A} \subseteq\left\{A_{1}, \ldots, A_{n}\right\}$. Assim:

$$
U \cap \Psi(\mathcal{A}) \subseteq\left\{A_{1}\right\} \cup A_{1} \cup \cdots \cup\left\{A_{n}\right\} \cup A_{n} \cup k
$$

Percebamos que o lado direito da desigualdade é compacto. Assim, tomando o fecho de ambos os lados, temos que $\operatorname{cl} U \subseteq \Psi(\mathcal{A})$, o que viola o fato de que $C \subseteq U$.

Analogamente, $V \cap \omega \in \mathcal{I}^{+}(\mathcal{A})$, logo, $\omega \backslash P \in \mathcal{I}^{+}(\mathcal{A})$

Antes de passarmos para a próxima seção, aproveitaremos para provar um fato interessante.

Proposição 5.5. Existe uma MAD family $\mathcal{A}$ tal que $\left|\Psi(\mathcal{A})^{*}\right| \geq 2^{\omega}$.

Demonstração. Nessa demonstração, identificaremos $\Psi(\mathcal{A})$ dentro de $\beta \Psi(\mathcal{A})$. Seja $\mathcal{B}$ uma MAD family de cardinalidade $2^{\omega}$. Para cada $B \in \mathcal{B}$, seja $\mathcal{A}_{B}$ uma MAD family em $A \subseteq \omega$, ou seja, $\mathcal{A}_{B}$ é uma coleção infinita quase disjunta de subconjuntos de $A$ maximal nesse sentido. Seja $\mathcal{A}=$ $\bigcup_{B \in \mathcal{B}} \mathcal{A}_{B}$

Para cada $B \in \mathcal{B}$, o conjunto $\mathcal{A}_{B} \cup B$ é um zero set. Para ver isso, seja $f: \Psi(\mathcal{A}) \rightarrow\{0,1\}$ dada por $f(x)=0$ para todo $x \in A_{B} \cup B$, e 1 nos demais pontos. Temos que $f$ é contínua em $\omega$, pois esse é discreto. Se $A \in \mathcal{A}_{B}$ temos que $f$ é constante e igual a 0 em $\{A\} \cup A \subseteq\{A\} \cup B$. Finalmente, se $A \in \mathcal{A}_{B^{\prime}}$ com $B \neq B^{\prime}$, temos que existe $n$ natural tal que $A \cap B=B^{\prime} \cap B \subseteq n$. Assim temos que em $\{A\} \cup A \backslash n, f$ é constante e igual à 1 . Logo, $f$ é contínua e $f^{-1}[\{0\}]=\mathcal{A}_{B} \cup B$. Seja $Z_{B}=\mathcal{A}_{B} \cup B$. 
Se $B \neq B^{\prime}$ são elementos de $\mathcal{B}$, temos que, em $\beta(\Psi(\mathcal{A})), \operatorname{cl} Z_{B} \cap \operatorname{cl} Z_{B^{\prime}}=\operatorname{cl} B \cap B^{\prime}=B \cap B^{\prime}$, que é finito. Se $B \in \mathcal{B}$, temos que $\mathcal{A}_{B} \subseteq \mathcal{A}$ é infinito, logo, possui um ponto de acumulação $x_{B} \in \beta(\Psi(\mathcal{A}))$. Temos que em $\Psi(\mathcal{A})$, qualquer subconjunto de $\mathcal{A}$ é fechado e discreto, logo, $x_{B} \in$ $\beta(\Psi(\mathcal{A})) \backslash \Psi(\mathcal{A})$. Temos então que $x_{B} \in \operatorname{cl} Z_{B} \backslash \Psi(\mathcal{A})$, e se $B \neq B^{\prime}$, segue que $x_{B} \neq x_{B}^{\prime}$, pois $\left(\operatorname{cl} Z_{B} \backslash \Psi(\mathcal{A})\right) \cap\left(\operatorname{cl} Z_{B^{\prime}} \backslash \Psi(\mathcal{A})\right)=B \cap B^{\prime} \cap \Psi(\mathcal{A})=\emptyset$. Como $|\mathcal{A}|=2^{\omega}$, segue que $\left\{x_{B}: B \in \mathcal{B}\right\}$ tem cardinalidade $\geq 2^{\omega}$.

\subsection{Unicidade de compactificação}

Uma pergunta interessante é quando que, em um espaço localmente compacto, $\beta X$ coincide com a compactificação por um ponto de $X$ (isso implica que $X$ possui uma única compactificação). Sabemos que isso não é verdade, por exemplo, para $X=\omega$. Porém é verdade para $X=\omega_{1}$. Diremos que uma MAD family cujo $\Psi$-espaço tem essa propriedade é uma família de Mrówka. Por enquanto, ainda não provamos a existência deste objeto, e a existência de um tal objeto é não trivial.

Definição 5.2. Uma MAD family $\mathcal{A}$ é uma família de Mrówka se $\left|\Psi(\mathcal{A})^{*}\right|=1$.

Antes de provar que existem tais famílias, iremos provar alguns fatos mais básicos de topologia geral que precisaremos utilizar.

Proposição 5.6. Seja $X$ um espaço hereditariamente Lindelöf. Então existe um aberto enumerável que contém qualquer aberto enumerável.

Demonstração. Seja $U=\bigcup\{A \subseteq X: A$ é aberto e $|A| \leq \omega\}$. Como $U$ é Lindelöf, existe uma coleção enumerável de abertos da coleção anterior cuja união é $U$, então $U$ é uma união enumerável de conjuntos enumeráveis, portanto, enumerável.

Corolário 5.7. Se $X$ é um espaço hereditariamente Lindelöf, existe uma quantidade coenumerável de pontos em $X$ de pontos tais que toda vizinhança de cada um deles é não enumerável.

Demonstração. Tome o complemento do $U$ da proposição anterior.

Corolário 5.8. Seja $X$ um espaço zero dimensional, Hausdorff e hereditariamente Lindelöf. Suponha que $F$ é um fechado não enumerável em $X$. Então $F$ pode ser particionado em dois fechados não enumeráveis.

Demonstração. Como $F$ é hereditariamente Lindelöf, pelo corolário anterior $F$ possui dois pontos tais que toda vizinhança, ao ser intersectada com $F$ é não enumerável. Tome um clopen que contém um desses pontos e não o outro. O clopen e seu complemento intersectados com $F$ atestam o enunciado.

Lema 5.9. Seja $X$ um espaço compacto tal que para todo $F$ fechado, se $F$ é não enumerável então existem $F_{1}, F_{2}$ não enumeráveis e fechados disjuntos com $F_{1}, F_{2} \subseteq F$. Então para todo fechado $F$, vale que $|F| \geq \mathfrak{c}$.

Demonstração. Utilizaremos um argumento árvore. Seja $T=\bigcup_{n \in \omega} 2^{n}$. Denotemos a concatenação de $s, t \in T$ por $s \frown t$. Procedemos recursivamente definindo $F_{s}$ para cada $s \in 2^{<\omega}$. Define-se que $F_{\emptyset}=F$. Definidos $F_{s}$ fechados não enumeráveis para todo $s \in 2^{n}$, tomam-se $F_{s \frown(0)}, F_{s} \frown(1)$ fechados não enumeráveis disjuntos contidos em $F_{s}$.

Para cada $f: \omega \rightarrow 2$, temos que $\left(F_{f \mid n}: n \in \omega\right)$ é uma sequência decrescente de fechados não vazios contidos em $F$, e se $f, g \in 2^{\omega}$ são distintos, as sequências são eventualmente disjuntas. Por compacidade, dada $f$, existe $x_{f} \in \bigcap\left\{F_{f \mid n}: n \in \omega\right\}$, e pelas observações anteriores, $\left\{x_{f}: f \in \omega\right\} \subseteq F$ e a função $f \rightarrow x_{f}$ é injetora, $\operatorname{logo}|F| \geq 2^{\omega}$.

Corolário 5.10. Seja $X$ um espaço compacto, hereditariamente Lindelöf, zero dimensional e Hausdorff. Então todo subconjunto fechado de $X$ tem cardinalidade $\geq 2^{\omega}$. Em particular, todo subconjunto fechado não enumerável de ${ }^{\omega} 2$ tem cardinalidade $2^{\omega}$. 
Proposição 5.11. Existem MAD families de cardinalidade $\mathfrak{c}$ sem particionadores não triviais.

Demonstração. Seja $\mathcal{C}=\left\{A_{f}: f \in{ }^{\omega} 2\right\}$, onde $A_{f}=\{f \mid n: n \in \omega\}$. Temos que $\mathcal{C}$ é uma almost disjoint family em $2^{<\omega}$ de cardinalidade $\mathfrak{c}$. Podemos estende-la à uma MAD family $\mathcal{B}_{0}$ em $2^{<\omega}$.

Pelo Lema 2.2, existe uma MAD family $\mathcal{B}_{1}=\left\{B_{f}: f \in{ }^{\omega} 2\right\}$ tal que se $f \neq g, B_{f} \neq B_{g}$ e $A_{f} \subseteq B_{f}$.

Afirmo que se $X \subseteq \mathcal{B}_{1}$ é particionador não trivial de $B_{0}$, então $\left|\left\{A \in \mathcal{B}_{1}: A \subseteq{ }^{*} X\right\}\right|=\mathfrak{c}$.

Com efeito, seja $Z=\left\{f \in \omega_{2}: A_{f} \subseteq^{*} X\right\}$. Como $X$ é particionador, basta ver que $Z$ tem cardinalidade $\mathfrak{c}$. Por 4.9 , temos que $\mathcal{B}_{1} \mid X$ é MAD family, logo, não enumerável, o que implica em $Z$ ser não enumerável. Notemos que $Z=\bigcup_{n \in \omega}\left\{f \in \omega_{2}: \forall m \geq n(f \mid m \in X)\right\}$. Assim, existe $n$ tal que $\left\{f \in \omega_{2}: \forall m \geq n(f \mid m \in X)\right\}$ é não enumerável. Esse conjunto é fechado: o seu complemento é $\left\{f \in{ }^{\omega} 2: \exists m \geq n(f \mid m \notin X)\right\}$, que é aberto, pois se $f$ está nesse conjunto, fixe $m$ tal que $f \mid m \notin X$. O conjunto $\left\{g \in \omega_{2}: g|m=f| m\right\}$ é uma vizinhança aberta de $f$ contida nele. Pelo corolário anterior, ele tem cardinalidade $\mathfrak{c}$. Logo, $|Z|=\mathfrak{c}$.

Enumeremos todos os particionadores não triviais de $\mathcal{B}_{1}$ como $\left\{P_{\alpha}: \alpha<\kappa\right\}$ sem repetição para algum $\kappa \leq \mathfrak{c}$.

Recursivamente, tomemos $A_{\alpha}, B_{\alpha} \in \mathcal{B}_{1} \backslash\left\{A_{\beta}, B_{\beta}: \beta<\kappa\right\}$ para $\beta<\kappa$ com $A_{\alpha} \subseteq^{*} P_{\alpha}$ e $B_{\alpha} \cap P_{\alpha}={ }^{*} \emptyset$. Seja:

$$
\mathcal{B}_{2}=\left\{A_{\alpha} \cup B_{\alpha}: \alpha<\kappa\right\} \cup\left(\mathcal{B}_{1} \backslash\left\{A_{\alpha}, B_{\alpha}: \alpha<\kappa\right\}\right) .
$$

Temos que $\mathcal{B}_{2}$ é uma MAD family. Afirmo que $\mathcal{B}_{2}$ não tem particionador não trivial: se $P$ fosse um particionador não trivial de $\mathcal{B}_{2}$, também seria de $\mathcal{B}_{1}$, logo existiria $\alpha<\kappa \operatorname{com} P=P_{\alpha}$, mas $A_{\alpha} \subseteq^{*} P_{\alpha}$ e $B_{\alpha} \cap P={ }^{*} \emptyset$, absurdo.

Proposição 5.12. Dada uma almost disjoint family $\mathcal{B}$ de cardinalidade $\mathfrak{c}$, existe uma almost disjoint family $\mathcal{A}$ tal que:

a) Se $P$ é particionador não trivial de $\mathcal{A}$, então também é de $\mathcal{B}$,

b) $|\mathcal{A}|=\mathfrak{c}$,

c) $\beta \Psi(\mathcal{A})$ é zero dimensional,

d) $\mathcal{A}$ é MAD se, e somente se, $\mathcal{B}$ é MAD.

Demonstração. Seja $\left\{f_{\alpha}: \alpha<\mathfrak{c}\right\} \subseteq[0,1]^{\omega}$ o conjunto de todas as funções de $\omega$ em $[0,1]$ que possuem extensão contínua de $\Psi(\mathcal{B})$ sobre $[0,1]$. Recursivamente para $\alpha<\mathfrak{c}$, podemos escolher $A_{\alpha}, B_{\alpha} \in \mathcal{B} \backslash\left\{A_{\beta}, B_{\beta}: \beta<\alpha\right\}$ tais que se $f_{\alpha}$ se estende para uma (única) função contínua e sobrejetora $F_{\alpha}$, então $F_{\alpha}\left(A_{\alpha}\right) \neq F_{\alpha}\left(B_{\alpha}\right)$. Então seja:

$$
\mathcal{A}=\left\{A_{\alpha} \cup B_{\alpha}: \alpha<\mathfrak{c}\right\} \cup \mathcal{B} \backslash\left\{A_{\alpha}, B_{\alpha}: \alpha<\mathfrak{c}\right\}
$$

Temos que $\mathcal{A}$ é uma almost disjoint family de cardinalidade $\mathfrak{c}$ tal que todo particionador não trivial de $\mathcal{A}$ também é de $\mathcal{B}$. Temos ainda que $\mathcal{A}$ é MAD se, e somente se, $\mathcal{B}$ for MAD. Temos que não existe $F: \Psi(\mathcal{A}) \rightarrow[0,1]$ sobrejetora: se existisse, seja $f=F \mid \omega$. Temos que definindo-se $f\left(A_{\alpha}\right)=f\left(B_{\alpha}\right)=F\left(A_{\alpha} \cup B_{\alpha}\right)$ para todo $\alpha<c$ e $f(A)=F(A)$ para todo $A \in \mathcal{B} \backslash\left\{A_{\alpha}, B_{\alpha}: \alpha<\mathfrak{c}\right\}$, $f: \Psi(\mathcal{B}) \rightarrow[0,1]$ é contínua e sobrejetora. Mas então existe $\alpha$ tal que $f=f_{\alpha}$, o que implica em $f\left(A_{\alpha}\right) \neq f\left(B_{\alpha}\right)$, absurdo. Logo, pela Proposição 1.55 , temos que $\beta(\Psi(\mathcal{A}))$ é zero-dimensional.

Corolário 5.13. Existem famílias de Mrowka de cardinalidade $\mathfrak{c}$.

Demonstração. Combinemos as duas últimas proposições e o Teorema 5.4. Como o remainder da MAD family obtida é conexo e totalmente desconexo, ele é unitário. 
À luz da proposição acima, alguém poderia perguntar-se se todo espaço topológico localmente compacto cuja compactificação de Stone-Čech coincide com a compactificação por um ponto é homeomorfo ao $\Psi$-espaço de alguma MAD family. A proposição abaixo mostra que não, já que $\omega_{1}$ não é separável, mas qualquer $\Psi$-espaço é.

Proposição 5.14. Seja $\omega_{1}$ munido da topologia da ordem. Então:

a) Toda função $f: \omega_{1} \rightarrow \mathbb{R}$ contínua é eventualmente constante, ou seja, $\exists \alpha<\omega_{1} \forall \beta>\alpha(f(\beta)=$ $f(\alpha))$.

b) A topologia da ordem de $\omega_{1}$ coincide com a topologia de subespaço de $\omega_{1}+1$, e $\omega_{1}$ é $C$-imerso em $\omega_{1}+1$. Além disso, $\omega_{1}$ é denso em $\omega_{1}+1$.

c) Ordinais sucessores são compactos.

d) $\beta \omega_{1} \approx \omega_{1}+1$ é a compactificação por um ponto de $\omega_{1}$.

Demonstração. a) Suponha que $f: \omega_{1} \rightarrow \mathbb{R}$ é contínua. Fixe $\epsilon>0$. Para todo $\alpha<\omega_{1}$ limite, existe $g(\alpha)<\alpha$ tal que para todo $\beta \in(g(\alpha), \alpha],|f(\beta)-f(\alpha)|<\frac{\epsilon}{2}$.

Notemos que nas condições acima, se $\beta_{1}, \beta_{2} \in(g(\alpha), \alpha]$ então $\left.\mid f\left(\beta_{1}\right)-f\left(\beta_{2}\right)\right)|\leq| f\left(\beta_{1}\right)-$ $f(\alpha)|+| f\left(\beta_{2}-f(\alpha) \mid<\epsilon\right.$.

Assim, define-se $g: S \rightarrow \omega_{1}$, onde $S$ é o conjunto dos ordinais limites enumeráveis, que é um club de $\omega_{1}$, portanto, estacionário. $g$ é tal que $\forall \alpha \in S(g(\alpha)<\alpha)$. Pelo Lema de Fodor (Lema $1.24)$, existe $\delta$ tal que $g^{-1}[\{\delta\}]$ é estacionário, portanto limitado. Assim, se $x, y \in\left(\delta, \omega_{1}\right)$, temos que $|f(x)-f(y)|<\epsilon$, pois existe $z>x, y$ tal que $z \in g^{-1}(\{\delta\})$.

Assim, conclui-se que dado $\epsilon>0$ existe $\delta_{\epsilon}<\omega_{1}$ tais que $\forall \alpha, \beta \in\left(\delta_{\epsilon}, \omega_{1}\right),|f(\alpha)-f(\beta)|<\epsilon$. Seja $\delta=\sup \left\{\delta_{\frac{1}{n}}: n \in \omega, n>1\right\}$. Temos que se $\alpha, \beta>\delta$ então $f(\alpha)=f(\beta)$. Para a última afirmação, como $\omega_{1}$ é limite, uma base local de $\omega_{1}$ é da forma $\left(\alpha, \omega_{1}\right]$ para $\alpha<\omega_{1}$.

b) É fácil ver que em ambas as topologias, 0 e os ordinais sucessores são discretos, e que dado $\gamma$ limite, os conjuntos do tipo $(\alpha, \gamma]$ para $\alpha<\gamma$ formam uma base local. Pelo item anterior, se $f: \omega_{1} \rightarrow \mathbb{R}$ é contínua, ela é eventualmente constante igual à algum $x$. Definindo-se $f\left(\omega_{1}\right)=x$, temos uma extensão contínua de $f$.

c) Seja $\beta=\alpha+1$ um ordinal sucessor e suponha que $\mathcal{A}$ é uma cobertura aberta de $\beta$ sem subcobertura finita. Seja $\alpha_{0}=\alpha$. Existe $U_{0} \in \mathcal{A}$ tal que $\alpha_{0} \in U_{0}$. Suponha que escolhemos uma sequência finita $U_{0}, \ldots, U_{n}$ de elementos de $\mathcal{A}$ e $\alpha_{0}>\alpha_{1}>\cdots>\alpha_{n}$ uma sequência estritamente decrescente de ordinais tal que $\left[\alpha_{n}, \beta\right) \subseteq \bigcup_{i=0}^{n} U_{i}$. Como a cobertura $\mathcal{A}$ não possui subcobertura finita, temos que $\beta \backslash \bigcup_{i=0}^{n} U_{i} \neq \emptyset$, e esse conjunto é fechado. Logo, seu supremo pertence à ele, e, portanto, é seu máximo. Seja $\alpha_{n+1}=\max \beta \backslash \bigcup_{i=0}^{n} U_{i}$. Temos que $\alpha_{n+1}<\alpha_{n}$ pois $\left[\alpha_{n}, \beta\right) \subseteq \bigcup_{i=0}^{n} U_{i}$. Tome $U_{n+1} \in \mathcal{A} \operatorname{com} \alpha_{n+1} \in U_{n+1}$. Por construção, temos que $\left(\alpha_{n+1}, \beta\right) \subseteq \bigcup_{i=0}^{n} U_{i}, \operatorname{logo},\left[\alpha_{n+1}, \beta\right) \subseteq \bigcup_{i=0}^{n+1} U_{i}$.

Dessa forma, constrói-se uma sequência infinita estritamente decrescente de ordinais, o que é absurdo já que todo conjunto de ordinais possui elemento mínimo.

d) Pelos itens anteriores, vimos que $\omega_{1}+1$ é uma compactificação de $\omega_{1}$ na qual $\omega_{1}$ é $C$-imerso (e, portanto, $C^{*}$-imerso). Logo, $\omega_{1}+1 \approx \beta \omega_{1}$. Como $\left|\omega_{1}+1 \backslash \omega_{1}\right|=1, \beta \omega_{1}$ é a compactificação por um ponto de $\omega_{1}$.

\subsection{Mais sobre famílias de Mrówka}

Nessa seção, daremos uma construção clássica de famílias de Mrówka. Para isso, seguiremos ideias dos artigos [Mro70] e [Mro77]. 
Definição 5.3. Vamos definir alguns subconjuntos de $\mathbb{R}^{2}$. Sejam $R=\{(x, 0): x \in \mathbb{R}\}, R_{n}=$ $\left\{\left(x, \frac{1}{n}\right): x \in \mathbb{R}\right\}$ para $n>0$ natural. Seja $X=\left\{\left(\frac{m}{2 n}, \frac{1}{n}\right): m \in \mathbb{Z}, n \in \mathbb{N}, n>0\right\} \cup R$. Para cada $n>1$ natural e $p \in \mathbb{R}$ seja $V_{n}(p)$ a interseção do triângulo equilátero fechado e massivo que tem vértice em $(p, 0)$ e um lado contido em $R_{k} \operatorname{com} X$, ou seja, $V_{n}(p)=\left\{\left(\frac{m}{2 k}, \frac{1}{k}\right): k \in \omega \backslash n, m \in \mathbb{Z},\left|\frac{m}{2 k}-p\right|<\right.$ $\left.\frac{1}{\sqrt{3} k}\right\}$.

Consideraremos $X$ como espaço topológico onde os pontos de $X \backslash R$ são discretos, e se $(p, 0) \in \mathbb{R}$ então as vizinhanças desse ponto são dadas por $V_{n}(p)$, para $n>1$. Fica a cargo do leitor verificar que isso de fato gera uma topologia Hausdorff e que cada um desses abertos é um compacto (portanto, fechado). Notemos ainda que se $p \neq q$ e $m, n>1$, então $V_{n}(p) \cap V_{m}(p) \subseteq X \backslash R$ é finito, e que dado $n>1$, então se $k \geq n$ temos que existe $m \operatorname{com}\left(\frac{m}{2 k}, \frac{1}{k}\right),\left(\frac{m+1}{2 k}, \frac{1}{k}\right) \in V_{n}(p) \cap R_{k}$ e $\frac{m}{2 k} \leq p \leq \frac{m+1}{2 k}$. Para verificar esse último fato, seja $m$ o maior inteiro tal que $\frac{m}{2 k} \leq p$. Temos que $p-\frac{m}{2 k}<\frac{1}{\sqrt{3} k}$, ou teríamos que $p-\frac{m}{2 k} \geq \frac{1}{2 k}$, e, assim, $p \geq \frac{m+1}{2 k}$. Temos que $\left|\frac{m+1}{2 k}-p\right|=\frac{m+1}{2 k}-p<\frac{m+1}{2 k}-\frac{m}{2 k}=\frac{1}{2 k}<\frac{1}{\sqrt{3} k}$.

Com isso, podemos provar a seguinte proposição:

Proposição 5.15. O espaço $X$ definido acima é tal que para toda $f: X \rightarrow \mathbb{R}$ contínua, a função $f \circ i_{0}$ é um limite pontual de funções contínuas de $\mathbb{R}$ em $\mathbb{R}$, onde $i_{0}: \mathbb{R} \rightarrow R$ é a bijeção dada por $i_{0}(x)=(x, 0)$.

Demonstração. Seja, para cada $n>1, i_{n}: \mathbb{R} \rightarrow R_{n}$ bijetora dada por $i_{0}(x)=\left(x, \frac{1}{n}\right)$ Para cada $n>1$, estendamos $f \mid\left(X \cap R_{n}\right)$ para $f \mid R_{n}$ de modo que em cada intervalo entre algum $\frac{m}{2 n}$ e $\frac{m+1}{2 n}$, de $R_{n} \backslash X$, definamos $f\left(x, \frac{1}{2 n}\right)=f\left(\frac{m}{2 n}, \frac{1}{n}\right)-f\left(\frac{m+1}{2 n}, \frac{1}{n}\right) \frac{x-\frac{m}{2 n}}{\frac{1}{2 n}}$. Assim, temos que $f_{n}=f \circ i_{n}$ é contínua. Afirmo que dado $x$ real, $f \circ i_{0}(x)=R=\lim _{n \rightarrow \infty} f_{n}(x)$. Com efeito, fixe $\epsilon>0$. Como $f$ é contínua em $x$, existe $N>0$ tal que $f\left[V_{N}(p)\right] \subseteq B(x, \epsilon)$. Como dado $n \geq N$ existe $m$ como no parágrafo precedente à proposição, pela definição da extensão de $f$, temos que se $n \geq N, f_{n}(x) \in B(x, \epsilon)$.

Corolário 5.16. Existe uma almost disjoint family $\mathcal{A}$ de cardinalidade $\mathfrak{c}$ tal que existe $h: \mathbb{R} \rightarrow \mathcal{A}$ bijetora tal que $\forall f: \Psi(\mathcal{B}) \rightarrow \mathbb{R}$ contínua, a função $f \circ h: \mathbb{R} \rightarrow \mathbb{R}$ é um limite de funções contínuas de $\mathbb{R}$ em $\mathbb{R}$.

Demonstração. Consideremos o espaço $X$ definido nessa seção. Construiremos uma almost disjoint family sobre o conjunto enumerável $N=X \backslash R$. Para cada $r \in \mathbb{R}$, seja $A_{r}=V_{1}(r) \backslash\{r\}$. Temos que $\mathcal{A}=\left\{A_{r}: r \in \mathbb{R}\right\}$ é uma MAD family em $N$ de cardinalidade $\mathfrak{c}$, e $u: X \rightarrow \Psi(\mathcal{A})$ dada por $u(n)=n$ para todo $n \in N, u(r, 0)=A_{r}$ para todo $r \in R$ é um homeomorfismo. Seja $h=u \circ i_{0}$. Se $f: \Psi(\mathcal{A}) \rightarrow \mathbb{R}$ é contínua, temos que $f \circ u$ é contínua, logo, pela proposição anterior, $f \circ u \circ i_{0}=f \circ h$ é limite pontual de funções contínuas de $\mathbb{R}$ em $\mathbb{R}$.

Proposição 5.17. Existe uma MAD family $\mathcal{B}$ de cardinalidade $\mathfrak{c}$ tal que existe $h: \mathbb{R} \rightarrow \mathcal{B}$ bijetora tal que $\forall f: \Psi(\mathcal{A}) \rightarrow \mathbb{R}$ contínua, a função $f \circ h: \mathbb{R} \rightarrow \mathbb{R}$ é um limite de funções contínuas de $\mathbb{R}$ em $\mathbb{R}$.

Demonstração. Pelo corolário anterior, existe uma almost disjoint family $\mathcal{A}$ de cardinalidade $\mathfrak{c}$ tal que existe $h: \mathbb{R} \rightarrow \mathcal{A}$ bijetora tal que $\forall f: \Psi(\mathcal{A}) \rightarrow \mathbb{R}$, a função $f \circ h: \mathbb{R} \rightarrow \mathbb{R}$ contínua é um limite de funções contínuas de $\mathbb{R}$ em $\mathbb{R}$. Pelo Lema 2.2, existe uma MAD family $\mathcal{B}=\left\{B_{A}: A \in \mathcal{B}\right\}$ tal que se $A \neq A^{\prime}$ então $B_{A} \neq B_{A^{\prime}}$ e tal que $A \subseteq B_{A}$ para todos $A, A^{\prime} \in \mathcal{A}$. Seja $i: \Psi(\mathcal{A}) \rightarrow \Psi(\mathcal{B})$ dada por $i(A)=B_{A}$ para todo $A \in \mathcal{A}$, e $i(n)=n$ para todo $n \in \omega$. Temos que $i$ é contínua. Agora seja $h_{0}=h \circ i$. Tome $f: \Psi(\mathcal{B}) \rightarrow \mathbb{R}$ contínua. Temos que $f \circ h_{0}=(f \circ i) \circ\left(i^{-1} \circ h_{0}\right)=(f \circ i) \circ h$. Como $f \circ i$ é contínua, segue a tese.

Lema 5.18. Seja $\mathcal{A}$ uma almost disjoint family com a propriedade do Corolário 5.16. Então se $Z \subseteq \mathcal{A} \subseteq \Psi(\mathcal{A})$ é um zero-set não enumerável, temos que $|Z|=\mathfrak{c}$. Além disso, se $\mathcal{A} \backslash Z$ é não enumerável, temos que $|\mathcal{A} \backslash Z|<\mathfrak{c}$. 
Demonstração. Suponha que $f^{-1}[\{0\}] \subseteq \mathcal{A}$ é um zero-set, com $f: \Psi(\mathcal{A}) \rightarrow[0,1]$ contínua. Então $g=f \circ h: \mathbb{R} \rightarrow \mathbb{R}$ é limite pontual de funções reais $f_{n}$. Como $F_{\sigma}$ 's e $G_{\delta}$ 's de $\mathbb{R}$ não enumeráveis têm cardinalidade $\mathfrak{c}$, basta observar que $g^{-1}[\{0\}]$ é um $G_{\delta}$ e que $g^{-1}[\mathbb{R} \backslash\{0\}]$ é um $F_{\sigma}$. Provaremos a segunda observação (a primeira decorre desta). Basta observar que:

$$
g^{-1}[\mathbb{R} \backslash\{0\}]=\bigcup_{m>0} \bigcup_{k>0} \bigcap_{n \geq k}\left\{x \in \mathbb{R}:\left|f_{n}(x)\right| \geq \frac{1}{m}\right\}
$$

Questões para se falar da compactificação de Stone-Čech de um $\Psi$-espaço são questões acerca de como caracterizam-se seus zero-sets. O teorema abaixo é um pequeno resultado nessa direção.

Proposição 5.19. Seja $\mathcal{A}$ uma MAD family. Então todo zero set $Z \subseteq \mathcal{A} \subseteq \Psi(\mathcal{A})$ infinito é não enumerável.

Demonstração. Temos que $\Psi(\mathcal{A})$ é um espaço de Tychonoff pseudocompacto (Proposição 2.22). Se $Z \subseteq \mathcal{A}$ é infinito enumerável, temos que $Z$ é infinito e discreto, logo, não é compacto. Portanto, pelo Corolário 1.59 , temos que $Z$ não é um zero set.

Lema 5.20. Seja $\mathcal{A}$ uma almost disjoint family. Então $\mathcal{A}$ é um zero-set de $\Psi(\mathcal{A})$.

Demonstração. A função $f: \Psi(\mathcal{A}) \rightarrow[0,1]$ dada por $f(n)=\frac{1}{2^{n}}$ para $n$ natural e $f(A)=0$ para $A \in \mathcal{A}$ é contínua.

Abaixo, resolveremos um exercício de [Mro77].

Proposição 5.21. Seja $\mathcal{A}$ uma almost disjoint family e $X=\Psi(\mathcal{A})$. São equivalentes:

a) $\mathcal{A}$ é maximal.

b) $\psi(\mathcal{A})$ é pseudocompacto.

c) Todo subconjunto infinito de $\omega$ possui um ponto de acumulação em $\mathcal{A}$.

d) $\beta X \backslash e[\omega]=\operatorname{cl} e[\mathcal{A}]$.

e) Para todo subconjunto fechado $B$ de $X$, temos que cl $e[B] \cap X^{*} \subseteq \operatorname{cl} e[B \cap \mathcal{A}]$.

Demonstração. A Proposição 2.22 nos diz que a) e b) são equivalentes.

c) é equivalente à a): Se $\mathcal{A}$ for maximal, dado $B \subseteq \omega$ infinito, temos que existe $A \in \mathcal{A}$ com $B \cap A$ infinito, assim, $A$ é ponto de acumulação de $B$. Reciprocamente, se $\mathcal{A}$ não for maximal, existe $B \subseteq \omega$ infinito tal que $B \cap A$ é finito para todo $A \in \mathcal{A}$. Temos que $B$ é fechado e discreto, logo, não possui ponto de acumulação.

Agora, veremos que $c \rightarrow d \rightarrow e$ e que $\neg a \rightarrow \neg e$. Suponha $c$ ). Como $e[\omega] \subseteq \beta X$ é aberto disjunto de $e[\mathcal{A}]$, temos que cl $e[\mathcal{A}] \subseteq \beta X \backslash e[\omega]$. Agora tome $p$ um z-ultrafiltro livre de $X$. O lema anterior nos diz que $\mathcal{A}$ é um zero set de $X$. Basta ver que $\mathcal{A} \in p$. Pela Proposição 1.44 (a), basta ver que se $B \in p$, então $B \cap \mathcal{A} \neq \emptyset$. Como $p$ é livre, dado $B \in p$, temos que $B$ é infinito. Por c), $B \cap \mathcal{A} \neq \emptyset$ (pois $B$ é fechado).

Agora suponha d). Suponha que $p$ é um ultrafiltro livre tal que $p \in \beta X \backslash \operatorname{cl} e[B \cap \mathcal{A}]$. Veremos que $p \in \beta X \backslash \operatorname{cle} e[B]$. Como $p \in \beta X \backslash \operatorname{cl} e[B \cap \mathcal{A}]$, existe $Z$ zero set tal que $B \cap \mathcal{A} \subseteq Z$ e $Z \notin p$. Podemos supor que $Z \subseteq \mathcal{A}$, pois $\mathcal{A}$ é um zero-set. Existe $W \in p$ com $W \cap Z=\emptyset$, e podemos supor que $W \subseteq \mathcal{A}$, pois, por $\mathrm{d}), \mathcal{A} \in p$.

Afirmo que $Z \cup B=Z \cup(B \cap \omega)$ é um zero set. Com efeito, seja $f: X \rightarrow \mathbb{R}$ tal que $Z=f^{-1}[\{0\}]$. Seja $g: X \rightarrow \mathbb{R}$ dada por $f(x)=0$ para todo $x \in Z \cup B$, e $f(x)=g(x)$ para todo $x \in X \backslash(Z \cup B)$. Como $Z \cup B$ é aberto, $g$ é contínua em $X \backslash(Z \cup B)$. Temos que em $B \cap \omega$, a função é contínua pois $\omega$ é discreto. Finalmente, se $x \in Z, g$ é contínua em $x$ pois se $V$ é vizinhança aberta de 0 , existe 
$U$ vizinhança aberta de $x \operatorname{com} f[U] \subseteq V$. Como $0 \in V$, segue que $g[U] \subseteq V . g$ atesta que $Z \cup B$ é zero-set.

Temos que $p \in \operatorname{cl} e[W]$, logo, como $W \cap(Z \cup B)=\emptyset$, temos que $p \notin \operatorname{cl} e[B] \subseteq \operatorname{cl} e[Z \cup B]$.

Finalmente, provaremos que $\neg a) \rightarrow \neg e$ ). Se $\mathcal{A}$ não for maximal, existe $B \subseteq \omega$ tal que $B \cap A$ é finito para todo $A \in \mathcal{A}$, e tal que $B$ é infinito.

Temos que $B$ é fechado em $X$, mas não é compacto, logo, cl $e[B] \cap X^{*} \neq \emptyset$. Porém, $\operatorname{cl} e[B \cap \mathcal{A}]=\emptyset$.

Lema 5.22. Seja $\mathcal{A}$ uma MAD family. Então se $Z \subseteq \mathcal{A}$ é tal que $|\mathcal{A} \backslash Z| \leq \omega$, então $Z$ é um zero-set de $\Psi(\mathcal{A})$.

Demonstração. Para cada $A \in \mathcal{A}$, seja $Z_{A}=\mathcal{A} \backslash\{A\}$. A função $g: \Psi(\mathcal{A}) \rightarrow[0,1]$ dada por $f(A)=1$, $f(x)=1$ para todo $x \in A, f(B)=0$ para todo $B \in \mathcal{A} \backslash\{A\}$ e $f\left(x_{n}\right)=\frac{1}{2^{n}}$, onde $\left\{x_{n}: n \in \omega\right\}=\omega \backslash A$, enumerado em ordem crescente, é uma função contínua que atesta que $Z_{A}$ é zero-set. Logo, dada uma sequência $\left\{A_{n}: n \in \omega\right\}$ de elementos de $\mathcal{A}$, temos que $\mathcal{A} \backslash\left\{A_{n}: n \in \omega\right\}=\bigcap_{n \in \omega}\left(\mathcal{A} \backslash\left\{A_{n}\right\}\right)$ é um zero-set.

Antes de demonstrar a próxima proposição, provaremos um lema apresentado por S. Mrówka em [Mro77].

Lema 5.23. ?? Seja $\mathcal{A}=\left(A_{i}: i \in I\right)$ uma família não vazia e $\kappa$ um cardinal infinito tal que $|I| \leq \kappa$ e para todo $i \in I,\left|A_{i}\right|=\kappa$. Então existe $\left(A_{i}^{\prime}: i \in I\right)$ uma família tal que para todo $i \in I, A_{i}^{\prime} \in\left[A_{i}\right]^{\kappa}$ e para todos $i, j \in I, A_{i} \cap A_{j}=\emptyset$.

Demonstração. Seja $f: \kappa \rightarrow \kappa \times I$ bijetora. Podemos escrever $f=(g, h)$, onde $g=\pi_{1} \circ f, h=\pi_{2} \circ f$. Para cada $\alpha<\kappa$, seja $x_{\alpha} \in A_{g(\alpha)} \backslash\left\{x_{\beta}: \beta<\alpha\right\}$. Por fim, seja $A_{i}^{\prime}=\left\{x_{\alpha}: g(\alpha)=i\right\}$.

Lema 5.24 (S. Mrówka). Sejam $\kappa$ um cardinal infinito e $\mathcal{F}$ uma coleção de funções de domínio $R$ tal que $|R|=\kappa$ e $|\mathcal{F}| \leq \kappa$. Então existe $\pi: R \rightarrow R$ bijetora tal que para toda $f \in \mathcal{F}$, se $f \circ \pi \in \mathcal{F}$ então existe $y$ tal que $\left|R \backslash f^{-1}[\{y\}]\right|<\kappa$, e tal que $\pi^{2}$ é a identidade em $R$.

Demonstração. Seja $\mathcal{F}_{0} \subseteq \mathcal{F}$ a coleção dos elementos $f$ de $\mathcal{F}$ tais que para todo $y,\left|R \backslash f^{-1}[\{y\}]\right|=\kappa$. Basta construir uma permutação $\pi$ em $R$ tal que se $f \in \mathcal{F}_{0}$, então $f \circ \pi \notin \mathcal{F}$. Enumeremos $\mathcal{F}_{0}=\left\{f_{\xi}: \xi \in I\right\}$. Temos que $|I| \leq \kappa$. Sejam:

$$
I_{1}=\left\{\xi \in I: f_{\xi} \text { possui uma fibra de cardinalidade } \kappa\right\}
$$

$$
I_{2}=I \backslash I_{1}
$$

Para cada $\xi \in I_{1}$, seja $A_{\xi}$ uma fibra de $f_{\xi}$ de cardinalidade $\kappa, B_{\xi}=R \backslash A_{\xi}$. Por construção, $A_{\xi}, B_{\xi}$ tem cardinalidade $\kappa$. Para cada $\xi \in I_{2}$, seja $C_{\xi}=R$ e $D_{\xi, y}=R \backslash f_{\xi}^{-1}[\{y\}]$ para todo $y \in f_{\xi}[R]$. Assim, definimos uma família de no máximo $\kappa$ conjuntos de cardinalidade $\kappa$. Pelo Lema ??, para esses respectivos índices, existem conjuntos dois a dois disjuntos $A_{\xi}^{\prime} \in\left[A_{\xi}\right]^{\kappa}, B_{\xi}^{\prime} \in\left[B_{\xi}\right]^{\kappa}$, $C_{\xi}^{\prime} \in\left[C_{\xi}\right]^{\kappa}, D_{\xi, y}^{\prime} \in\left[D_{\xi, y}\right]^{\kappa}$.

Definiremos nossa bijeção nesses pedaços disjuntos de $R$. Dado $\xi \in I_{1}$, para cada $K \subseteq A_{\xi}^{\prime}$ seja $\pi_{K}$ uma permutação em $A_{\xi}^{\prime} \cup B_{\xi}^{\prime}$ tal que $\pi_{K}^{2}(x)=x$ e tal que $\pi_{K}(x)=x$ para todo $x \in K$ e tal que $\pi_{k}(x) \in B_{\xi}^{\prime}$ para todo $x \in B_{\xi}^{\prime}$. Note que se $K, K^{\prime} \subseteq A_{\xi}^{\prime}$ são distintos, então temos que $f_{\xi} \circ \pi_{K}$ e $f_{\xi} \circ \pi_{K^{\prime}}$ são distintos. Como existem $2^{\kappa}$ subconjuntos de $A_{\xi}^{\prime}$, existe $\pi_{\xi}$ permutação nesse domínio tal que $f_{\xi} \circ \pi_{\xi} \neq f \mid\left(A_{\xi}^{\prime} \cup B_{\xi}^{\prime}\right)$ para toda $f \in \mathcal{F}$.

Agora, fixe $\xi \in I_{2}$. Para cada $y$, seja $\kappa_{y}=\left|f_{\xi}^{-1}\left[\{y\} \cap C_{\xi}^{\prime}\right]\right|<\kappa$. Seja $E_{\xi}=f_{\xi}\left[C_{\xi}^{\prime}\right]$. Seja $C_{\xi}^{\prime \prime} \subseteq C_{\xi}^{\prime}$ tal que $f_{\xi} \mid C_{\xi}^{\prime \prime}$ seja injetora e que $f\left[C_{\xi}^{\prime \prime}\right]=E_{\xi}$. Para cada $y \in E_{\xi}$, seja $x_{y}$ o único elemento de $C_{\xi}^{\prime \prime}$ tal que $f_{\xi}\left(x_{y}\right)=y$. Seja $D=\prod_{y \in E_{\xi}} D_{\xi, y}^{\prime}$. Sendo $\kappa_{y}^{\prime}=\kappa$ para todo $y$, temos que, pela desigualdade de König:

$$
|D|=\prod_{y \in E_{\xi}} \kappa_{y}^{\prime}>\sum_{y \in E_{\xi}} \kappa_{y}=\left|C_{\xi}^{\prime}\right|=\kappa
$$


Para cada $d \in D$, seja $d=\left(d_{y}\right)_{y \in E_{y}}$. Seja $\pi_{d}$ a permutação em $C_{\xi}^{\prime \prime} \cup\left\{D_{\xi, y}^{\prime}: y \in E_{\xi}\right\}$ dada por $\pi_{d}\left(x_{y}\right)=d_{y}, \pi_{d}\left(d_{y}\right)=x_{y}$ para todo $y \in E_{\xi}$, e que age como a identidade nos demais pontos. Se $d, e \in D$ são distintos, então $f_{\xi} \circ \pi_{d} \neq f_{\xi} \circ \pi_{e}$. Como $|D|>\kappa$, existe $\pi_{\xi}$ permutação nesse domínio tal que $f_{\xi} \circ \pi_{\xi} \neq f \mid\left(C_{\xi}^{\prime \prime} \cup \bigcup_{y \in E_{\xi}} D_{\xi, y}^{\prime}\right)$ para cada $f \in \mathcal{F}$.

Unindo-se todas as $\pi_{\xi}$ construídas e completando com a identidade, segue a tese.

Proposição 5.25. Na notação da Proposição 2.31, considerando os $\Psi$-espaços $\mathcal{A} \cup P, \mathcal{B} \cup Q$, se $\mathcal{A}, \mathcal{B}$ possuem as propriedades da Proposição 5.17, então para todo $f$, se $E \subseteq \mathcal{A}_{f}$ é infinito, então $E \subseteq \mathcal{A}_{f}$ é um zero-set se, e somente se, $\mathcal{A} \backslash E$ é enumerável.

Demonstração. Seja $\mathcal{F}$ a coleção de todas as funções $g$ de $\mathcal{A}$ em $\mathbb{R}$ que admitem uma extensão contínua $\tilde{g}: \Psi(\mathcal{A}) \rightarrow \mathbb{R}$. Como a transformação $g \rightarrow \tilde{g} \mid P$ é injetora, temos que $|\mathcal{F}| \leq \mathfrak{c}$. Além disso, $|\mathcal{A}|=\mathfrak{c}$. Logo, pelo Lema 5.24, existe $\pi$ bijeção em $\mathcal{A}$ com $\pi^{2}$ sendo a identidade em $\mathcal{A}$ tal que se $g, g \circ \pi \in \mathcal{A}$ então existe $y \in \mathbb{R}$ tal que $\left|\mathbb{R} \backslash g^{-1}[\{0\}]\right|<\mathfrak{c}$.

Fixe $f^{\prime}$, e seja $f=f^{\prime} \circ \pi^{-1}$. Então $f^{\prime}=f \circ \pi$. Suponha que $E=g^{-1}[\{0\}] \subseteq \mathcal{A}_{f \circ \pi}$ é um zero-set.

Seja $\tilde{g}: \mathcal{A} \rightarrow \mathbb{R}$ dada por $\tilde{g}(A)=g(A \cup f \circ \pi(A))$. Temos que, definindo-se $\tilde{g}(n)=g(n)$ para $n \in$ $P$, temos que $\tilde{g}: \mathcal{A} \cup P \rightarrow \mathbb{R}$ é contínua. Logo, $\tilde{g} \in \mathcal{F}$. Além disso, $u(A)=\tilde{g} \circ \pi(A)=g(\pi(A) \cup f(A))$. Definindo-se $u(n)=g \circ f(n)$ para $n \in P$, temos que $u$ é contínua. Assim, $\tilde{g} \circ \pi \in \mathcal{F}$. Logo, existe $y$ tal que $\left|\mathcal{A} \backslash \tilde{g}^{-1}[\{y\}]\right|<\mathfrak{c}$.

Temos que $\left|\mathcal{A}_{f \circ \pi} \backslash E\right|=\left|\mathcal{A} \backslash \tilde{g}^{-1}[\{0\}]\right|$, pois $A \cup f \circ \pi(A) \rightarrow A$ é uma bijeção entre esses dois conjuntos. Da mesma forma, $\left|g^{-1}[\{0\}]\right|=\left|\tilde{g}^{-1}[\{0\}]\right|$. Pela Proposição 5.19, $E$ é não enumerável, e pelo Lema 5.18, $E$ tem cardinalidade c. Então $y=0$. Mas como $\mid \mathcal{A} \backslash g^{-1}[\{0\}]$ é um cozero-set, novamente pelo Lema $5.18,\left|\mathcal{A}_{f \circ \pi} \backslash E\right|=\left|\mathcal{A} \backslash \tilde{g}^{-1}[\{0\}]\right| \leq \omega$.

A recíproca decorre do lema anterior.

Utilizando a proposição 2.31 e a proposição acima, obtemos o seguinte corolário:

Corolário 5.26. Existem $2^{\mathfrak{c}}$ MAD families cujos $\Psi$-espaços não são homeomorfos tais que todo zero set infinito contido na MAD family é co-enumerável. Além disso, dada uma partição de $\omega$ por dois subconjuntos infinitos, podemos supor que cada elemento de cada uma dessas MAD families intersecta infinitamente ambos os elementos da partição.

Corolário 5.27. Existem $2^{\mathfrak{c}}$ MAD families cujos $\Psi$-espaços não são homeomorfos tais que suas compactificações de Stone-Čech coincidem com suas compactificações por um ponto.

Demonstração. Basta observar que se $p, q$ são ultrafiltros livres distintos em um $\Psi$-espaço de uma MAD family, então eles devem conter zero sets disjuntos contidos na MAD family.

\subsection{Espaços métricos compactos e remainders de $\Psi$-espaços}

Nessa seção, mostraremos que todo espaço métrico compacto sem pontos isolados é homeomorfo a um remainder de um $\Psi$-espaço de uma MAD family. Como referência, citamos o artigo [Ter80].

Proposição 5.28. Sejam $X$ pseudocompacto e $Y$ um espaço métrico compacto. Seja $f: X \rightarrow Y$ contínua. Então $\beta X=\bigcup\left\{\operatorname{cl} e\left[f^{-1}[\{y\}]\right]: y \in Y\right\}$.

Demonstração. Tome $p \in \beta X$. Como $X$ é pseudocompacto, $p$ tem a propriedade da interseção enumerável. Seja $F: \beta X \rightarrow Y$ contínua tal que $F \circ e=f$. Afirmo que $F(p) \in \bigcap\{\operatorname{cl} f[Z]: Z \in p\}$. Com efeito, dado $Z \in p$, temos que $p \in \operatorname{cl} e[Z]$. Assim, existe $\left(x_{\lambda}\right)_{\lambda \in \Lambda}$ rede em $Z$ tal que $e\left(x_{\lambda}\right) \rightarrow p$. Logo, $f\left(x_{\lambda}\right)=F \circ e\left(x_{\lambda}\right) \rightarrow F(p)$. Mas $f\left(x_{\lambda}\right)$ é uma rede em $f[Z]$, o que mostra que $F(p) \in \operatorname{cl} f[Z]$. Para $n>0$ natural, seja $U_{n}$ a bola fechada de centro $F(p)$ e raio $\frac{1}{n}$. Fixe $Z \in p$. Como $U_{n}$ é vizinhança de $F(p) \in Z$, temos que $U_{n} \cap f[Z] \neq \emptyset$. Logo, $f^{-1}\left[U_{n}\right] \cap Z \neq \emptyset$. Como $Z$ é arbitrário, temos que $f^{-1}\left[U_{n}\right] \in p$. Como $n$ é arbitrário e $p$ possui a propriedade da interseção enumerável, temos que $f^{-1}[\{F(p)\}]=\bigcap_{n \in \omega} f^{-1}\left[U_{n}\right] \neq \emptyset$. Devemos ter que $f^{-1}[\{F(p)\}] \in p$, ou existe $W \in p$ disjunto desse conjunto, o que implica que $\bigcap_{n \in \omega} f^{-1}\left[U_{n}\right] \cap W=\emptyset$, absurdo. Assim, temos que $f^{-1}[\{F(p)\}] \in p$, portanto $p \in \operatorname{cl} f^{-1}[\{F(p)\}]$. Segue a tese. 
Teorema 5.29. Seja $X$ um espaço metrizável compacto sem pontos isolados. Existe uma MAD family $\mathcal{A}$ de cardinalidade $\mathfrak{c}$, tal que $\psi(\mathcal{A})^{*} \approx X$.

Demonstração. Como $X$ é compacto e metrizável, temos que $X$ é separável. Seja $N \subseteq X$ enumerável denso. Como $X$ não tem pontos isolados, $N$ é infinito. Sem perda de generalidade, podemos supor que nenhum subconjunto infinito de $N$ é um elemento de $N$ (pois $X$ é homeomorfo a um espaço topológico cujo conjunto base é um cardinal, e $\omega=N)$.

Particionando $N$ em infinitos subconjuntos infinitos, temos que cada um desses conjuntos contém uma subsequência convergente injetora (pois $X$ é sequencialmente compacto). Logo, pelo Lema de Zorn, existe $\mathcal{A}_{1}$ maximal em

$$
P=\{\mathcal{B} \subseteq \mathscr{P}(X): \forall A \in \mathcal{B}(A \text { é a imagem de uma sequência convergente injetora }) \wedge|\mathcal{B}| \geq \omega
$$

$$
\wedge \forall A, B \in \mathcal{B}(A \neq B \rightarrow|A \cap B|<\omega)\}
$$

Temos que $\mathcal{A}_{1}$ é uma MAD family, pois se existir $B \subseteq N$ tal que $\left|B \cap A_{1}\right|<\omega$ para todo $A \in \mathcal{A}$, temos que existe $B^{\prime} \subseteq B$ imagem de uma sequência convergente injetora, e temos que $\mathcal{A}_{1} \cup\left\{B^{\prime}\right\} \in P$.

Dado $x \in X$, seja $\mathcal{A}_{1}(x)$ a coleção de todos os elementos de $\mathcal{A}_{1}$ que são imagens de uma sequência que converge para $x$. Temos que $\mathcal{A}_{1}(x) \neq \emptyset$, caso contrário, a imagem de uma sequência injetora em $N$ que converge para $x$ violaria a maximalidade de $\mathcal{A}_{1}$. Para cada $x$, escolhamos $\lambda(x) \in \mathcal{A}_{1}(x)$. No conjunto infinito enumerável $\lambda(x)$, existe uma MAD family de cardinalidade $\mathfrak{c}, \mathcal{A}_{2}(x)$, tal que todo zero-set em seu $\Psi$-espaço contido em $\mathcal{A}_{2}(x)$ é co-enumerável (em particular, todo zero-set não enumerável de $\mathcal{A}_{2}(x)$ tem cardinalidade $\left.\mathfrak{c}\right)$. Seja:

$$
\mathcal{A}_{3}(x)=\left(\mathcal{A}_{1}(x) \backslash\{\lambda(x)\}\right) \cup \mathcal{A}_{2}(x) .
$$

Seja $i_{x}: \mathcal{A}_{3}(x) \backslash \mathcal{A}_{2}(x) \rightarrow \mathcal{A}_{2}(x)$ injetora. Seja:

$$
\mathcal{A}_{4}(x)=\left(\mathcal{A}_{2}(x) \backslash \operatorname{ran}\left(i_{x}\right)\right) \cup\left\{\lambda \cup i_{x}(\lambda): \lambda \in \mathcal{A}_{3}(x) \backslash \mathcal{A}_{2}(x)\right\}
$$

Temos que $\mathcal{A}_{4}=\bigcup_{x \in X} \mathcal{A}_{4}(x)$ é uma MAD family de elementos de $N$. Seja $\mathcal{F}$ a coleção de todas as funções contínuas $f: N \cup \mathcal{A}_{4} \rightarrow[0,1]$ tais que existem $t, t^{\prime} \in[0,1]$ distintos e $x \in X$ tais que ambos $f^{-1}[\{t\}] \cap \mathcal{A}_{4}(x)$ e $f^{-1}\left[\left\{t^{\prime}\right\}\right] \cap \mathcal{A}_{4}(x)$ são infinitos. Como $N$ é enumerável denso, temos que $|\mathcal{F}| \leq \mathfrak{c}$. sendo $\tau$ a cardinalidade de $\mathcal{F}$, escrevamos $\mathcal{F}=\left\{f_{\alpha}: \alpha<\tau\right\}$ injetivamente. Para cada $\alpha<\tau$, sejam $t_{\alpha} \neq t_{\alpha}^{\prime} \in[0,1]$ e $x_{\alpha} \in X$ tais que $f_{\alpha}^{-1}\left[\left\{t_{\alpha}\right\}\right] \cap \mathcal{A}_{4}\left(x_{\alpha}\right)$ e $f_{\alpha}^{-1}\left[\left\{t_{\alpha^{\prime}}\right\}\right] \cap \mathcal{A}_{4}\left(x_{\alpha}\right)$ são infinitos. Temos que esses conjuntos são zero sets, pois cada $\mathcal{A}_{4}(x)$ é um zero set já que a função $g: N \cup\left\{\mathcal{A}_{4}\right\} \rightarrow X$ que fixa $N$ e leva elementos de $\mathcal{A}_{4}(x)$ em $x$ é contínua, e $\mathcal{A}_{4}$ é um zero set (pelo Lema 5.20).

Considere $k: \Psi\left(\mathcal{A}_{2}\left(x_{\alpha}\right)\right) \rightarrow \Psi\left(\mathcal{A}_{4}\right)$ que fixa $N$ e tal que $k(\lambda)=\lambda$, se $\lambda \in \mathcal{A}_{2}\left(x_{\alpha}\right) \backslash \operatorname{ran} i_{x_{\alpha}}$, e $k\left(i_{x_{\alpha}}(\lambda)\right)=i(\lambda) \cup \lambda$ caso $i_{x_{\alpha}}(\lambda) \in \mathcal{A}_{2}\left(x_{\alpha}\right)$. Temos que $k$ é uma contínua e sobrejeta $N \cup A_{4}\left(x_{\alpha}\right)$, logo, dado $\alpha$, pela propriedade de $\mathcal{A}_{2}\left(x_{\alpha}\right)$, temos que os seguintes conjuntos tem cardinalidade $\mathfrak{c}$ :

$$
\begin{aligned}
& k^{-1}\left(f_{\alpha}^{-1}\left[\left\{t_{\alpha}\right\}\right] \cap \mathcal{A}_{4}\left(x_{\alpha}\right)\right)=\left(f_{\alpha} \circ k\right)^{-1}\left[\left\{t_{\alpha}\right\}\right] \\
& k^{-1}\left(f_{\alpha}^{-1}\left[\left\{t_{\alpha}^{\prime}\right\}\right] \cap \mathcal{A}_{4}\left(x_{\alpha}\right)\right)=\left(f_{\alpha} \circ k\right)^{-1}\left[\left\{t_{\alpha}^{\prime}\right\}\right]
\end{aligned}
$$

Assim:

$$
\left|f_{\alpha}^{-1}\left[\left\{t_{\alpha}^{\prime}\right\}\right] \cap \mathcal{A}_{4}\left(x_{\alpha}\right)\right|=\left|f_{\alpha}^{-1}\left[\left\{t_{\alpha}^{\prime}\right\}\right] \cap \mathcal{A}_{4}\left(x_{\alpha}\right)\right|=\mathfrak{c}
$$

Procedendo por indução transfinita, podemos escolher pontos $\lambda_{\alpha} \neq \mu_{\alpha} \in \mathcal{A}_{4}\left(x_{\alpha}\right)$ tais que $f_{\alpha}\left(\lambda_{\alpha}\right) \neq f_{\alpha}\left(\mu_{\alpha}\right)$ para $\alpha<\tau$, todos distintos, e definir:

$$
\mathcal{A}_{5}=\left(\mathcal{A}_{4} \backslash\left\{\lambda_{\alpha}, \mu_{\alpha}: \alpha<\tau\right\}\right) \cup\left\{\lambda_{\alpha} \cup \mu_{\alpha}: \alpha<\tau\right\}
$$


Temos que $\mathcal{A}_{5}$ é uma MAD family de cardinalidade $\mathfrak{c}$.

Seja $h: \Psi\left(\mathcal{A}_{5}\right) \rightarrow X$ a função contínua que fixa $N$ e leva $A \in \mathcal{A}_{5}$ no limite da sequência de imagem $A$. Temos que $h$ é contínua. Seja $h^{\prime}: \beta \Psi\left(\mathcal{A}_{5}\right) \rightarrow X$ contínua tal que $h^{\prime} \circ e=h$. Seja $g=h^{\prime} \mid \Psi\left(\mathcal{A}_{5}\right)^{*}$. Veremos que $g: \Psi\left(\mathcal{A}_{5}\right)^{*} \rightarrow X$ é homeomorfismo.

Para cada $x$, temos que $\mathcal{A}_{5}(x)=\left\{\lambda \in \mathcal{A}_{5}: h(\lambda)=x\right\}$ é infinito, fechado e discreto em $\Psi\left(\mathcal{A}_{5}\right)$. Logo, e $\left[\mathcal{A}_{5}(x)\right]$ possui um ponto de acumulação em $\Psi\left(\mathcal{A}_{5}\right)^{*}$, e a imagem desse ponto por $g$ é $x$. Logo, $g$ é sobrejetora.

Pelo lema anterior, $\beta \Psi\left(\mathcal{A}_{5}\right)=\bigcup_{x \in X}$ cle $[\mathcal{A}(x)]$. Para ver que $g$ é injetora, basta mostrar que $\operatorname{cl} \mathcal{A}_{5}(x) \backslash e\left[\Psi\left(\mathcal{A}_{5}\right)\right]$ é unitário. Suponha que existam $p, q$ dois ultrafiltros distintos nesse conjunto. Tomemos $Z \in p, W \in q$ zero sets disjuntos contidos em $\mathcal{A}_{5}(x)$. Seja $f: \Psi\left(\mathcal{A}_{5}\right) \rightarrow[0,1]$ contínua tal que $f^{-1}[\{0\}]=Z, f^{-1}[\{1\}]=W$. Podemos definir $\tilde{f}: \Psi\left(\mathcal{A}_{4}\right) \rightarrow[0,1]$ dada por $\tilde{f}(x)=f(x)$ se $x \in N \cup\left(\mathcal{A}_{4} \backslash\left\{\lambda_{\alpha}, \mu_{\alpha}: \alpha<\tau\right\}\right)$, e $\tilde{f}\left(\lambda_{\alpha}\right)=\tilde{f}\left(\mu_{\alpha}\right)=f\left(\lambda_{\alpha} \cup \mu_{\alpha}\right)$ para $\alpha<\tau$. Temos que $\tilde{f}$ é contínua, logo, existe $\alpha$ tal que $\tilde{f}=f_{\alpha}$. Mas então $\tilde{f}\left(\lambda_{\alpha}\right) \neq \tilde{f}\left(\mu_{\alpha}\right)$, absurdo.

Resta apenas ver que $g$ é contínua. Mas isso ocorre pois $g$ é uma restrição de função contínua. Como os espaços são compactos, decorre que a inversa é contínua e segue a tese. 


\section{Capítulo 6}

\section{MAD families e os racionais}

\subsection{Introdução}

Neste capítulo, apresentaremos resultados do artigo [Hru01], bem como diversos pré-requisitos e noções necessárias para bem compreende-lo. As definições de árvore aqui dadas não são definições gerais, mas são suficientes e bastante intuitivas para o contexto em que iremos trabalhar. Além disso, as definições de $\mathbb{Q}$ aqui dadas, bem como as funções auxiliares que se relacionam com $\mathbb{Q}$, se aplicam apenas a este capítulo.

Neste capítulo, discutiremos a noção de destrutibilidade de um ideal alto e de MAD families, noções de forcing, e, em particular, o Forcing de Sacks.

Definição 6.1. Seja $I \subseteq \omega$ um ideal alto e livre, $M$ uma interpretação transitiva e enumerável de uma teoria estendendo ZFC e $P \in M$ um forcing poset. Dizemos que $I$ é $P$-indestrutível se para todo $G P$-genérico sobre $M, I$ é alto em $M[G]$. Caso contrário, $I$ é dito $P$-destrutível. Dizemos que uma MAD family $\mathcal{A}$ é $P$-indestrutível (destrutível) se $I(\mathcal{A})$ for $P$-indestrutível (destrutível), ou, equivalentemente, se $\mathcal{A}$ for $\operatorname{MAD}$ em $M[G]$ (não for mad).

\section{2 Árvores em $2^{<\omega}$}

Definição 6.2. Se $p \subseteq 2^{<\omega}$, define-se $[p]=\left\{f \in 2^{\omega}: \forall n \in \omega \exists s \in p(f \mid n \subseteq s)\right\}$. Notemos que se $M$ é uma interpretação transitiva de $Z F C$ tal que $p \in M$, então $[p]^{M}=[p] \cap M$. [ $[p]$ é o conjunto (fechado) codificado por $p$, e dizemos que $p$ codifica $[p]$.

Se $F \subseteq 2^{\omega}$, seja $C_{F}=\{f \mid n: f \in F, n \in \omega\}$. $C_{F}$ é o codificador do fecho de $F$. Notemos que se $F \in M$ é um fechado de $\left(2^{\omega}\right)^{M}$, então $\left(C_{F}\right)^{M}=C_{F}$. Seja $I(F)$ o conjunto dos pontos isolados de $F$.

Se $p \subseteq 2^{<\omega}$, seja o conjunto dos ramos isolados de $p$ o conjunto $I(p)=\left\{s \in p: \forall r, r^{\prime} \in p((p \subseteq\right.$ $\left.\left.r \wedge p \subseteq r^{\prime}\right) \rightarrow\left(r \subseteq r^{\prime} \vee r^{\prime} \subseteq r\right)\right)$. Notemos que se $p \in M, I^{M}(p)=I(p)$. Seja $B(p)=p \backslash I(p)$, e note que $B^{M}(p)=B(p)$ sempre que $p \in M$.

Lema 6.1. Se $p \subseteq 2^{<\omega}$, então $[p]$ é fechado. Se $F \subseteq 2^{\omega},\left[C_{F}\right]=\operatorname{cl} F$.

Demonstração. Suponha que $f \in 2^{\omega} \backslash p$. Existe $n \in \omega$ tal que $\forall s \in p, f \mid n \nsubseteq \subseteq s$. Logo, $V=\left\{g \in 2^{\omega}\right.$ : $g|n=f| n\}$ é uma vizinhança aberta de $f$ disjunta de $[p]$, o que mostra que $[p]$ é fechado.

Para a segunda afirmação, dado $f \in F$,para todo $n \in \omega$ temos que $f \mid n \in p$, $\operatorname{logo}, f \in\left[C_{F}\right]$. Assim, $F \subseteq C_{F}$. Como $\left[C_{F}\right]$ é fechado, temos que cl $F \subseteq F$. Para a inclusão contrária, fixe $f \in\left[C_{F}\right]$. Temos que dado $n \in \omega$ existe $g_{n} \in F \operatorname{com} g_{n}|n=f| n$. Assim, temos que $f \in \operatorname{cl}\left\{g_{n}: n \in \omega\right\} \subseteq$ cl F.

O lema e a definição acima nos dizem como codificar subconjuntos fechados de $2^{\omega}$ a partir de subconjuntos de $2^{<\omega}$. Utilizar tais codificações nos trará vantagens para lidar com relativizações a modelos transitivos de ZFC. Abaixo, definiremos noções relacionadas a àrvores. Tais noções não são 
tão gerais como poderiam ser, pois trataremos apenas de sub-árvores de $2^{<\omega}$. Para um tratamento mais geral sobre árvores, ver [Kun80] ou [Kun11].

Definição 6.3. Dizemos que $p \subseteq 2^{<\omega}$ é uma árvore se dado $s \in p$ e $n \in \mathbb{N}, s \mid n \in p$.

Dizemos que uma árvore $p$ é bem podada se dado $s \in p$, se $k>\operatorname{dom} p$ então existe $t \in 2^{k} \cap s$ com $s \subseteq t$.

Dizemos que uma árvore $p$ é perfeita se dado $s \in p$, existe $n \in \mathbb{N}$ e $t, t^{\prime} \in 2^{n} \cap p$ distintos com $s \subseteq t, t^{\prime}$.

Notemos que toda árvore perfeita é bem podada e que as noções acima são todas absolutas para interpretações transitivas de ZFC.

Dado $p \subseteq 2^{\omega}$, o conjunto tree $(p)=\left\{s \in 2^{<\omega}: \exists r \in p(s \subseteq r)\right\}$ é a árvore gerada por $p$. Notemos que tree $(p)$ é absoluto para interpretações transitivas de ZFC, e que tree $(p)$ é a menor árvore contendo $p$. Seja $\operatorname{prun}(p)=\left\{s \in p: \forall k>\operatorname{dom}(p) \exists t \in p \cap 2^{<\omega}(s \subsetneq t)\right\}$ a poda de $p$. Notemos que $\operatorname{prun}(p)$ é absoluto para interpretações transitivas de ZFC e é a maior árvore bem podada contida em $p$.

O conjunto de todas as árvores perfeitas não vazias, denotado por $\mathbb{S}$, ordenado pela inclusão, é chamado de Forcing de Sacks, cujo maior elemento é $2^{<\omega}$.

Se $p \in \mathbb{S}, s \in p$, define-se $p \mid s=\{t \in p: p \subseteq s$ ou $s \subseteq t\}$. Também define-se $\operatorname{Br}(p)=\{t \in p:$ $t \frown(0) \in p \wedge t \frown(1) \in p\}$ e $\operatorname{Br}_{n}(p)=\{t \in \operatorname{Br}(p):|\{s \in \operatorname{Br}(p): s \subseteq t\}|=n\}$

Intuitivamente, todas as árvores "nascem" do conjunto vazio, árvores bem podadas são árvores cujos ramos "nunca morrem", e árvores perfeitas são árvores tais que todos os ramos eventualmente sofrem uma divisão além de qualquer ponto dado.

Lema 6.2. Sejam $p \subseteq 2^{<\omega}$. Temos que $[p]=[\operatorname{tree}(p)]$. Se, além disso, $p$ é uma árvore, $[p]=[\operatorname{prun}(p)]$. Em particular, dado $p,[p]=[\operatorname{prun} \operatorname{tree}(p)]$, o que mostra que dado $p \subseteq 2^{<\omega}$ existe uma árvore bem podada $t$ tal que $[p]=[t]$.

Demonstração. Seja $p \subseteq 2^{<\omega}$. Como $p \subseteq \operatorname{tree}(p)$, temos que $[p] \subseteq[\operatorname{tree}(p)]$. Agora suponha que $f \in[\operatorname{tree}(p)]$. Queremos ver que dado $n \in \omega$, existe $s \in p$ com $f \mid n \subseteq s$. Sabemos que existe $t \in \operatorname{tree}(p) \operatorname{com} f \mid n \subseteq t$. Como $t \in \operatorname{tree}(p)$, temos que existe $s \in p$ com $t \subseteq s$, assim segue a tese.

Agora suponha que $p$ é uma árvore. Como $\operatorname{prun}(p) \subseteq p$, temos que $[\operatorname{prun}(p)] \subseteq[p]$. Agora fixe $f \in[p]$. Temos que dado $n \in \omega, f \mid n \in \omega$. Pela definição de $\operatorname{prun}(p)$, temos que para todo $n \in \omega$, $f \mid n \in \operatorname{prun}(p), \operatorname{logo}, f \in[\operatorname{prun}(p)]$.

O nome "árvore perfeita" não é por acaso: há uma relação entre árvores perfeitas e conjuntos perfeitos. Lembremos que um conjunto perfeito de um espaço topológico é um conjunto fechado sem pontos isolados.

Lema 6.3. Temos que $P \subseteq 2^{\omega}$ é perfeito se, e somente se, existe $p \in \mathbb{S}$ tal que $P=[p]$.

Demonstração. Primeiro, vejamos que se $p \in \mathbb{S},[p]$ é perfeito. Já sabemos que $[p]$ é fechado.

$[p]$ não tem pontos isolados: dado $f \in[p]$, uma vizinhança básica de $f$ é da forma $V=\left\{g \in 2^{\omega}\right.$ : $f \mid n \subseteq g\}$ para algum $n$ natural. Temos que $f \mid n \in p$, logo existe $k_{0}>n$ e $p_{0} \in 2^{k_{0}} \cap p$ com $f \mid n \subseteq p_{0}$ e tal que $p_{0}$ é incompatível com $f$. Definidos $k_{0}<k_{1}<\cdots<k_{m}$ e $p_{0} \subseteq \cdots \subseteq p_{m} \operatorname{com} p_{i} \in p \cap 2^{k_{i}}$, existe $k_{m+1}$ natural tal que existe $p_{m+1} \in p \cap 2^{k_{m+1}}$. Seja $g=\bigcup_{m \in \omega} p_{m}$. Temos que $g \in[p] \cap V$ e $g \neq f$.

Reciprocamente, suponha que $P$ é um conjunto perfeito e seja $p=C_{P}$. É claro que se $s \in p$ e $n \in \omega, s \mid n \in p$. Dado $f \mid n \in p$ com $f \in P$ e $n$ natural, existe $g \in P \cap\left\{h \in 2^{\omega}: f \mid n \subseteq h\right\}$ com $g \neq f$, pois $P$ não tem pontos isolados. Existe $k \geq n$ com $f(k) \neq g(k)$. Temos que $f|(k+1), g|(k+1)$ são dois elementos de $p$ distintos que contém $f \mid n$. Isso mostra que $p \in \mathbb{S}$. Finalmente, lembremos que $[p]=\left[C_{P}\right]=P$.

Seguindo a notação do artigo [Hru01], vamos identificar $\mathbb{Q}$ com um subconjunto de $2^{\omega}$ da seguinte forma: 
Definição 6.4. Nessa seção, $\mathbf{0} \in 2^{\omega}$ é a função nula, $\mathbb{R}=2^{\omega}$ e $\mathbb{Q} \subseteq 2^{\omega}$ é o conjunto das funções de suporte finito não nulas (ou seja, $f \in \mathbb{Q} \leftrightarrow|\{n \in \omega: f(n)=1\}|<\omega$ ). Se $q \in \mathbb{Q}, s_{q}=q \mid n$, onde $n=$ $\max \{k \in \omega: f(k)=1\}$. Se $s \in 2^{<\omega}, q(s)=s^{\frown}(1) \frown \mathbf{0} \in 2^{\omega}$. Se $p \in \mathbb{S}$, seja $\mathbb{Q}_{p}=\{q(s): s \in \operatorname{Br}(p)\}$.

Na notação acima, ordena-se $\mathbb{Q}$ fazendo que $q_{s} \frown(1) \leq q_{r} \frown(1)$ se, e somente se, $s \subseteq r$. Considera-se $\mathbb{Q}_{p} \subseteq \mathbb{Q}$ com a ordem de subespaço.

A ideia é que $\mathbb{Q}$ está identificado com $2^{<\omega}$ pelas funções inversas $q \rightarrow s_{q}$ e $s \rightarrow q(s)$. A ideia de remover a função nula de $\mathbb{Q}$ é ter como diferenciarmos os racionais induzidos, por exemplo, pelas sequências finitas $(0,1,0,0)$ e $(0,1)$ : o último 1 do racional demarca o seu fim, servindo como um separador entre a sequência que o induz e a sequência infinita de zeros.

Abaixo, provaremos alguns fatos sobre os racionais.

Lema 6.4. Sejam $p, q \in \mathbb{S}$. $\mathbb{Q}_{p}$ é ordem-isomorfo a $\mathbb{Q}$ e $p \leq q$ se, e somente se, $\mathbb{Q}_{p} \subseteq \mathbb{Q}_{q}$. Além disso, $\left(\operatorname{cl} \mathbb{Q}_{p}\right) \backslash \mathbb{Q}_{p} \subseteq[p]$.

Demonstração. Temos que $\mathbb{Q}=\left\{q(s): s \in 2^{<\omega}\right\}$. Escolhe-se recursivamente $t_{\sigma} \in \operatorname{Br}(p)$ para $\sigma \in 2^{<\omega}$ como se segue: $t_{\emptyset}$ é o elemento de $\operatorname{Br}(p)$ de menor domínio (e este é único: Se $n$ é o menor número natural tal que existem $t \in \operatorname{Br}(p) \cap 2^{n}$, se existirem $t, t^{\prime}$ distintos nesse conjunto, seja $k$ o maior natural tal que $t\left|k=t^{\prime}\right| k$. Temos que $0 \leq k<n$ e $t \mid k \in \operatorname{Br}(p)$. Isso viola a minimalidade de $n$ ). Definido $t_{\sigma}$, seja $t_{\sigma}-(0)$ o menor elemento de $\operatorname{Br}(p) \cap\left\{t \in p: t \geq t_{\sigma}\right\}$. Vejamos que ele está bem definido: sabemos que esse conjunto é não vazio. Sendo $n$ o primeiro natural tal $\operatorname{Br}(p) \cap\left\{t \in p: t \geq t_{\sigma}(0)\right\} \cap 2^{n}$ é não vazio, afirmo que $\operatorname{Br}(p) \cap\left\{t \in p: t \geq t_{\sigma}^{-}(0)\right\} \cap 2^{n}$ é unitário. Caso não seja, tomemos $t, t^{\prime}$ nesse conjunto. Sendo $k$ o maior natural tal que $t\left|k=t^{\prime}\right| k$, temos que $t_{\sigma}^{-}(0) \subseteq t\left|k, t^{\prime}\right| k, k<n$ e $t \mid k \in \operatorname{Br}(p) \cap\left\{t \in p: t \geq t_{\sigma}(0)\right\}$, o que viola a minimalidade de $n$. Logo, $t_{\sigma \frown(0)}$ está bem definido. Analogamente, define-se $t_{\sigma \frown(1)}$ como sendo o menor elemento de $\operatorname{Br}(p) \cap\left\{t \in p: t \geq t_{\sigma}(1)\right\}$

Agora considere $f: \mathbb{Q} \rightarrow \mathbb{Q}_{p}$ dado por $f(q(s))=q\left(t_{s}\right)$.

Temos que se $q(r) \leq q(s)$, então $r \subseteq s$ e então, por construção, $t_{r} \subseteq t_{s}$, assim, $f(q(r)) \leq f(q(s))$. Da mesma forma, se $q_{r \frown 1}<q_{s \frown 1}$ então $f(q(r \frown 1))<f(q(s))$. Agora suponha que $q(r), q(s)$ são incompatíveis. Então temos que $r, s$ são incompatíveis. Seja $n$ o maior natural tal que $r|n=s| n=l$. Sem perda de generalidade, $r(n)=0, s(n)=1$. Temos que $l^{\frown}(0) \subseteq t_{r}$ e $l^{\frown}(1) \subseteq t_{s}$. Logo, $t_{r}, t_{s}$ são incompatíveis, o que implica em $f(q(r)), f(q(s))$ serem incompatíveis. Isso mostra que $f$ preserva a ordem e é injetora. Resta apenas ver que $f$ é sobrejetora. Suponha que não seja, e seja $n$ o menor natural para o qual $\operatorname{Br}(p) \cap 2^{n} \backslash\left\{t_{\sigma}: \sigma \in 2^{\omega}\right\} \neq \emptyset$, e tomemos $t$ nessa diferença. Temos que $t$ não é o mínimo de $\operatorname{Br}(p)$, pois este é $t_{\emptyset}$. Logo, seja $t_{\sigma}$ o maior elemento de $\operatorname{Br}(p)$ estritamente abaixo de $t$. Por construção, temos que $t=t_{\sigma \frown(0)}$ ou $t=t_{\sigma \frown(1)}$, o que é absurdo.

Para a segunda afirmação, basta notar que $p \subseteq q$ se, e somente se, $\operatorname{Br}(p) \subseteq \operatorname{Br}(q)$, o que ocorre se, e somente se, $\mathbb{Q}_{p} \subseteq \mathbb{Q}_{q}$. A única implicação que pode causar dúvidas é a de que $\operatorname{Br}(p) \subseteq \operatorname{Br}(q)$ implica que $p \subseteq q$. Ora, se $p \backslash q \neq \emptyset$, tomando $t$ na diferença, existe $s \geq t$ com $s \in \operatorname{Br}(\mathrm{p})$, e $s \notin q$, $\operatorname{logo}, s \notin \operatorname{Br}(q)$.

Para a terceira afirmação, suponha que $s \in\left(\operatorname{cl} \mathbb{Q}_{p}\right) \backslash \mathbb{Q}_{p}$. Queremos ver que dado $n, s \mid n \in p$. Dado $n$, seja $m>n+1$ tal que existe $k$ com $n+1<k<m$ tal que $s(k)=1$, o que é possível pois $s \notin \mathbb{Q}_{p}$. Temos que $\left\{r \in 2^{\omega}: s \mid m \subseteq r\right\} \cap \mathbb{Q}_{p} \neq \emptyset$. Tome $r$ nesse conjunto. $r$ é da forma $q_{t} \frown(1)$ para algum $t \in \operatorname{Br}(p)$. Logo, $s \mid m \subseteq t \frown(1) \frown \mathbf{0}$. Isso implica que $s \mid n \subseteq t$, caso contrário teremos que $\mathbf{0}(k)=1$. Como $s \mid n \subseteq t \in p$, temos que $s \mid n \in p$ e segue a tese.

Lema 6.5. Seja $A \subseteq \mathbb{Q}$. Se $\operatorname{cl} A$ é não enumerável, então existe $B \subseteq \mathbb{Q}$ ordem-isomorfo à $\mathbb{Q}$. Reciprocamente, se $A$ contém um subconjunto ordem-isomorfo à $\mathbb{Q}$, então cl $A$ é não enumerável.

Demonstração. Seja $p=\left\{s_{q}: q \in A\right\}$. Primeiramente, notemos que Dado $s \in 2^{<\omega}$, seja $B(s)=$ $\{x \in[p]: s \subseteq x\}$.

Primeiramente, vejamos que $\operatorname{cl} A \backslash \mathbb{Q} \subseteq[p] \subseteq \operatorname{cl} A$. Para a primeira inclusão, fixe $x \in \operatorname{cl} A \backslash \mathbb{Q}$. Dado $n \in \omega$, seja $m>n$ tal que $x|m \backslash x| n$ assume o valor 1 duas vezes. Existe $q \in \mathbb{A}$ tal que $q|m=x| m$, e, pela escolha de $m$, segue que $s_{q}|n=x| n$. Logo, $x \in[p]$. Para a segunda inclusão, se 
$x \in[p]$, dado $n$, temos que existe $m \geq n$ tal que $x \mid m \in p$, mas então existe $q \in A$ tal que $x \mid n \subseteq s_{q}$, logo, existe $q \in A$ tal que $x \mid n \subseteq q$, e segue a tese.

Em particular, $|[p]|=\mathfrak{c}$.

Veremos agora que dado $s \in 2^{<\omega}$ existe $t \in 2^{<\omega}$ tal que $s \subseteq t$ e $\left|B\left(t^{\frown}(0)\right)\right|=\left|B\left(t^{\frown}(1)\right)\right|=\mathfrak{c}$. Pois suponha que não. Definiremos $g$ sequência em $\{0,1\}$ como se segue: Definido $g(i) \forall i<n$ de modo que $|B(s \frown g \mid n)|=\mathfrak{c}$, seja $g(n)$ tal que $\left|B\left(s^{\frown} g \mid(n+1)\right)\right|=\mathfrak{c}$. Seja $h=1-g$. Por construção, para todo $n|B(s \frown g \mid n)| \leq \omega$. Mas temos que $B(s) \subseteq \bigcup_{n \geq 0} B(s \frown g \mid n \frown(h(n))) \cup\{s \frown g\}$, mas este segundo conjunto é enumerável, absurdo.

Agora provaremos o seguinte lema: Se $|B(s)|=\mathfrak{c}$, então existem $t_{0}, t_{1} \supsetneq s$ incompatíveis em $p$ tais que $\left|B\left(t_{0}\right)\right|=\left|B\left(t_{1}\right)\right|=\mathfrak{c}$. Pelo parágrafo anterior, existe $t \in 2^{<\omega}$ tal que $s \subseteq t$ e $\left|B\left(t^{\frown}(0)\right)\right|=$ $\left|B\left(t^{\frown}(1)\right)\right|=\mathfrak{c}$. Dado $i \in\{0,1\}$, existe $t_{i} \supset s^{\frown} t^{\frown}(i)$ com $t_{i} \in p$ tal que $\left|B\left(t_{i}\right)\right|=\mathfrak{c}$.

Seja $s_{\emptyset} \in p$ tal que $|B(s)|=\mathfrak{c}$ (o que existe pois $p$ é enumerável). Definido $s_{\sigma}$ para algum $\sigma \in 2^{<\omega}$ de modo que $\left|B\left(s_{\sigma}\right)\right|=\mathfrak{c}$, tomemos $s_{\sigma \frown(0)}, s_{\sigma \frown(1)}$ incompatíveis contendo $s_{\sigma}$ tais que, para $i \in\{0,1\},\left|B\left(s_{\sigma}^{-}(i)\right)\right|=\mathfrak{c}$ (o que existe pelo lema acima).

Temos que $\left\{q\left(s_{\sigma}\right): \sigma \in 2^{<\omega}\right\}$ é um subconjunto de $A$ ordem-isomorfo à $\mathbb{Q}$.

Para a recíproca, se $B \subseteq A$ é ordem-isomorfo à $\mathbb{Q}$, então $p=\operatorname{tree}\left\{s_{b}: b z \in B\right\}$ é uma árvore perfeita e $[p] \subseteq \operatorname{cl} A$.

Lema 6.6. Seja $B \subseteq \mathbb{Q}_{p}$. Temos que se $\mathrm{cl} B$ é não enumerável, então existe $q \subseteq p$ árvore perfeita tal que $[q] \subseteq \operatorname{cl} B$ e para todo $x \in[q]$ e $n \in \omega$ existe $m \geq n$ tal que $q(x \mid m) \in B$.

Demonstração. Seja $A \subseteq \mathbb{B}$ ordem isomorfo à $\mathbb{Q}$, e seja $q=\operatorname{tree}\left\{s_{x}: x \in A\right\}$. Temos que $q$ é uma árvore perfeita contida em $p$. Se $x \in[q]$, dado $n$ existe $y \in A$ tal que $s_{y} \supset x \mid n$, logo, $y \supset x \mid n$, e, assim, $x \in \operatorname{cl} A$. Logo, $[q] \subseteq \operatorname{cl} A \subseteq \operatorname{cl} B$. Para verificarmos a última propriedade, tome $x \in[q]$ e $n \in \omega$. Por construção, existe $y \in A$ e $m \geq n$ tal que $x \mid m=s_{x}$. Daí, $q(x \mid m)=y \in A \subseteq B$, e segue a tese. de $2^{\omega}$.

Agora vamos começar a definir a decomposição de Cantor-Bendixon de um fechado enumerável

Lema 6.7. Suponha que $p \subseteq 2^{\omega}$ seja uma árvore bem podada. $[p \backslash I(p)]=[p] \backslash I([p])$.

Demonstração. Suponha que $f \in[p \backslash I(p)]$. Temos que $f \in[p]$ pois $p \backslash I(p) \subseteq p$. Veremos que $f \notin I([p])$. Suponha por absurdo que $f \in I([p])$. Então existe $n \in \omega$ tal que $V=\left\{g \in 2^{\omega}: g|n=f| n\right\}$ é tal que $V \cap[p]=\{f\}$. Temos que se $m \geq n$ então $f \mid m \in I(p)$, caso contrário teríamos que existe $l>m$ tal que existe $g \in p \cap 2^{l}$ incompatível com $f$. Seja $g_{l}=g$. Definido $g_{i}$ para $l \leq i<\omega$, seja $g_{i+1} \geq g_{i}$ com $g_{i+1} \in 2^{i} \cap p$, o que é possível pois $p$ é uma árvore bem podada. Temos que $\bigcup_{i>l} g_{i} \neq f$ está em $V$, absurdo. Como $m$ é arbitrário, temos que $f \notin[p \backslash I(p)]$, absurdo.

Para a outra inclusão, suponha que $f \in[p] \backslash I([p])$. Queremos ver que se $n \in \omega$, então $f \mid n \in$ $p \backslash I(p)$. Sabemos que $f \mid n \in p$. Resta ver que $f \mid n \notin I(p)$. Como $f \notin I([p])$, existe $g \in[p]$ tal que $g|n=f| n$ e $g \neq f$. Existe $k>n$ tal que $f(k)=g(k)$. Temos que $f \mid(k+1)$ e $g \mid(k+1)$ atestam que $f \mid n \notin I(p)$.

Proposição 6.8. Seja $M$ uma interpretação transitiva para $Z F C$ e $p \in M$ tal que $p \subseteq 2^{<\omega}$. Então $I([p])=I^{M}\left([p]^{M}\right)$, ou seja, o conjunto dos pontos isolados gerados por $p$ é absoluto.

Demonstração. Podemos supor que $p$ é uma árvore bem podada.

Notemos que se $A \in M, A \subseteq\left(2^{\omega}\right)^{M}$ e $x \in I^{M}(A)$, temos que existe $V \in M$ vizinhança básica de $x$ tal que $V \cap A=\{x\}$, logo, $x \in I(A)$. Assim, $I^{M}(A) \subseteq I(A)$. Temos que $I^{M}\left([p]^{M}\right) \subseteq$ $I\left([p]^{M}\right)=I([p] \cap M) \subseteq I([p])$. Precisamos verificar essa última inclusão. Suponha que $x \in I([p] \cap M)$. Existe $n$ tal que $\{y \in[p] \cap M: x|n=y| n\}=\{x\}$. Afirmo que $\{y \in[p]: x|n=y| n\}=\{x\}$. Caso contrário, existe $y \neq x$ nesse conjunto. Existe $m>n$ tal que $y(m) \neq x(m)$. Temos que $y \mid(m+1) \in M$. Existe, em $M$, uma função $z \in 2^{\omega}$ contendo $y \mid(m+1)$, o que viola o fato de que $\{y \in[p] \cap M: x|n=y| n\}=\{x\}$. Logo, $\{y \in[p]: x|n=y| n\}=\{x\}$, e, assim, $x \in I([p])$. 
Para a inclusão contrária, fixe $f \in I([p])$. Existe $n$ tal que $V=\left\{g \in 2^{\omega}: f|n=g| n\right\}$ é tal que $V \cap[p]=\{f\}$. Afirmo $f \mid n \in I(p)$, pois suponha o contrário. Temos que existe $s$ incompatível com $f$ tal que $f \mid n \subseteq s$ e $s \in p$. Então, Sendo $\operatorname{dom} s=k$, define-se recursivamente $s_{k}=k$ e, definido $s_{l}$ para algum $k \leq l<\omega$, define-se $s_{l+1} \in p \cap 2^{l+1}$ acima de $s_{l}$. Temos que $\bigcup_{l \geq k} g_{k} \in[p] \cap(V \backslash\{f\})$, absurdo.

Assim, $f \mid n \in I(p)$. Logo, $C=\{s \in p: f \mid n \subseteq s\}$ é tal que $f=\bigcup C$. Por absolutidade, temos que $C \in M$, logo, $f \in M$, logo, $f \in[p]^{M}$ e $\left\{g \in\left(2^{\bar{\omega}}\right)^{M}: f|n=g| n\right\} \in M$ atesta que $f \in I^{M}\left([p]^{M}\right)$.

Definição 6.5. Suponha que $F \subseteq 2^{\omega}$. Define-se recursivamente para $\alpha$ ordinal:

$$
I_{\alpha}(F)=I\left(F \backslash \bigcup_{\beta<\alpha} I_{\beta}(F)\right)
$$

Suponha que $p \subseteq 2^{<\omega}$. Define-se $p_{\alpha}$ para $\alpha \in M$ ordinal recursivamente por: $p_{0}=\operatorname{prun}$ tree $(p)$, $p_{\alpha+1}=\operatorname{prun} \operatorname{tree}\left(p_{\alpha} \backslash I\left(p_{\alpha}\right)\right)$ e, para $\gamma<\alpha$ limite, $p_{\gamma}=$ prun tree $\left(\bigcap_{\alpha<\gamma} p_{\gamma}\right)$. Está claro que essa definição é absoluta para interpretações transitivas de ZFC.

Lema 6.9. Seja $p \subseteq 2^{<\omega}$. Então para todo $\alpha,\left[p_{\alpha}\right]=[p] \backslash \bigcup_{\beta<\alpha} I_{\beta}([p])$.

Demonstração. Para $\alpha=0$, temos que $\left[p_{0}\right]=[\operatorname{prun} \operatorname{tree}(p)]=[p]$. Supondo que a afirmação vale para $\alpha$, temos que

$$
\begin{gathered}
{\left[p_{\alpha+1}\right]=\left[p_{\alpha} \backslash I\left(p_{\alpha}\right)\right]=\left[p_{\alpha}\right] \backslash I\left(\left[p_{\alpha}\right]\right)=\left([p] \backslash \bigcup_{\beta<\alpha} I_{\beta}([p])\right) \backslash I\left([p] \backslash \bigcup_{\beta<\alpha} I_{\beta}([p])\right)} \\
=\left([p] \backslash \bigcup_{\beta<\alpha} I_{\beta}([p])\right) \backslash I_{\alpha}([p])=[p] \backslash \bigcup_{\beta \leq \alpha} I_{\beta}([p]) .
\end{gathered}
$$

Supondo que $\gamma$ seja limite e seja $f \in\left[p_{\gamma}\right]$. É claro que $f \in[p]$. Fixe $\beta<\gamma$. Temos que $f \notin I_{\beta}([p])$ pois $I_{\beta}([p]) \cap\left[p_{\beta+1}\right]=\emptyset$ e $\left[p_{\gamma}\right] \subseteq\left[p_{\beta}\right]$. Logo, vale a inclusão $\left[p_{\gamma}\right] \subseteq[p] \backslash \bigcup_{\beta<\gamma} I_{\beta}([p])$. Reciprocamente, suponha que $f \in 2^{\omega}\left[p_{\gamma}\right]$. Então existe $n$ tal que $f \mid n \notin p_{\gamma}$. Logo, existe $\beta<\gamma$ tal que $f \mid n \notin p_{\beta}$. Logo, $f \notin\left[p_{\beta}\right]=[p] \backslash \bigcup_{\delta<\beta} I_{\delta}([p])$. Logo, $f \notin[p] \backslash \bigcup_{\delta<\gamma} I_{\delta}([p])$.

Por indução, segue a tese.

Proposição 6.10. Seja $M$ uma interpretação transitiva de ZFC e suponha que $p \subseteq 2^{<\omega}$ é tal que $p \in M$. Então para todo $\alpha \in M$ ordinal, temos que $I_{\alpha}^{M}\left([p]^{M}\right)=I_{\alpha}([p])$.

Demonstração. Provaremos a afirmação do enunciado recursivamente. Basta observar que, supondo que a tese vale para todo $\beta<\alpha$ :

$$
I_{\alpha}^{M}\left([p]^{M}\right)=I^{M}\left(\left([p] \backslash \bigcup_{\beta<\alpha} I_{\beta}([p])\right)^{M}\right)=I^{M}\left(\left[p_{\alpha}\right]^{M}\right)=I\left(\left[p_{\alpha}\right]\right)=I_{\alpha}([p])
$$

Proposição 6.11. Suponha que $p \subseteq 2^{<\omega}$ é tal que $[p]$ é enumerável. Então:

1. $I_{0}([p])$ é não vazio.

2. Existe $\alpha<\omega_{1}$ tal que $I_{\alpha}([p])=\emptyset$. Seja $\alpha_{p}$ o primeiro.

3. $[p]=\bigcup_{\alpha<\alpha_{p}} I_{\alpha}([p])$ e essa união é disjunta.

4. $\alpha_{p}$ é absoluto para interpretações transitivas de ZFC. Ou seja, se $M$ é interpretação transitiva de ZFC, $p \in M$ e $(|[p]| \leq \omega)^{M}$ então $\alpha_{p}^{M}=\alpha_{p}$. 
Demonstração. Lembremos que se $F \subseteq 2^{\omega}$ é fechado e não possui pontos isolados, então $F$ possui cardinalidade c. Assim, $[p]$ possui pontos isolados. Logo, segue 1. Notemos que os $I_{\alpha}([p])$ são subconjuntos de $[p]$ dois a dois disjuntos, logo, existe $\alpha<\omega_{1}$ tal que $I_{\alpha}([p])=\emptyset$. Isso prova 2 . Agora suponha por absurdo que $\left[p_{\alpha_{p}}\right]=[p] \backslash \bigcup_{\alpha<\alpha_{p}} I_{\alpha_{p}}([p]) \neq \emptyset$. Esse conjunto é enumerável, portanto, temos que $I_{\alpha_{p}}([p])=I\left(\left[p_{\alpha_{p}}\right]\right) \neq \emptyset$, o que é absurdo. Logo, segue 3. O último item segue da absolutidade de $I_{\alpha}([p])$ para $[p]$ enumerável.

A decomposição do item 3. é a decomposição de Cantor-Bendixon de $[p]$.

Corolário 6.12. Seja $M$ uma interpretação transitiva de ZFC e suponha que $p \subseteq 2^{<\omega}$ é tal que $p \in M$ é tal que $[p]^{M}$ é enumerável em $M$. Então $[p]=[p]^{M}$. Além disso, se $A \subseteq 2^{\omega}$ é tal que $A \in M$ e $\mathrm{cl}^{M} A$ é enumerável em $M$, então $\mathrm{cl}^{M} A=\operatorname{cl} M$.

Demonstração.

$$
[p]^{M}=\bigcup_{\alpha<\alpha_{p}^{M}} I_{\alpha}^{M}\left([p]^{M}\right)=\bigcup_{\alpha<\alpha_{p}} I_{\alpha}([p])=[p] .
$$

Para a segunda afirmação, temos que $\operatorname{cl}^{M} A=[A]^{M}=[A]=\operatorname{cl} A$.

\subsection{O Forcing de Sacks}

Lema 6.13. Se $M$ é uma interpretação transitiva e enumerável de ZFC, $\mathbb{S}^{M}=\mathbb{S} \cap M$ é uma noção de forcing não trivial sobre $\mathbb{S}^{M}$.

Demonstração. Primeiro, notemos que pela absolutidade da definição de $\mathbb{S}$, temos que $\mathbb{S}^{M}=\mathbb{S} \cap M$. Pelo Lema 1.66, é suficiente provar que se $p \in \mathbb{S} \cap M$ é uma árvore perfeita, então $p$ contém duas árvores perfeitas incompatíveis. Tome $s \in \operatorname{Br}_{1}(p)$ qualquer e considere $p \backslash\left\{t \in 2^{<\omega}: s \frown(0) \subseteq t\right\}$ e $p \backslash\left\{t \in 2^{<\omega}: s \frown(1) \subseteq t\right\}$. Por absolutidade, esses dois conjuntos são árvores perfeitas em $M$, e são incompatíveis.

Lema 6.14. Se $M$ é uma interpretação transitiva e enumerável de ZFC e $G$ é $\mathbb{S}^{M}$-genérico sobre $M$, então:

$$
f=\bigcup \bigcap G
$$

é uma função de $\omega$ em 2 .

Além disso, $f \notin M$ e $G=\left\{p \in \mathbb{S}^{M}:\{f \mid n: n \in \omega\} \subseteq p\right\}$.

Demonstração. Se $r, s \in \bigcap G$, devemos ter que $r, s$ são compatíveis, pois caso sejam incompatíveis, o conjunto $D=\left\{p \in \mathbb{S}^{M}: r \notin p \vee s \notin p\right\} \in M$ é denso (verificaremos a seguir), logo, como $D \cap G$ é não vazio, ambas as funções não podem estar em $\bigcap G$. Para ver que $D$ é denso, seja $p$ uma árvore perfeita com $r, s \in p$ e seja $n$ o maior natural tal que $r|n=s| n=t$. Temos que $t \in \operatorname{Br}(p)$, e $p \backslash\left\{l \in 2^{<\omega}: t \frown(0) \subseteq l\right\} \in D$.

Assim, $f$ é função. Queremos ver que $\operatorname{dom} f=\omega$. Dado $n \in \omega$, deve existir $s \in 2^{n}$ tal que $t \in \bigcap G$, caso contrário, dado $s \in 2^{n}$, seja $t_{s}$ tal que $s \notin t_{s}$. Temos que $\left\{t_{s}: s \in 2^{n}\right\} \subseteq G$ é um subconjunto finito do filtro $G$ sem extensão comum, pois uma tal extensão não possui elementos de $2^{n}$, o que é absurdo.

Para a segunda afirmação, se $f \in M$, teríamos que o conjunto $\left\{p \in \mathbb{S}^{M}: \exists n \in \omega(f \mid n \notin p)\right\} \in M$ é denso: Se $p \in M \cap \mathbb{S} \backslash D$, temos que existe $n$ tal que $f \mid n \in \operatorname{Br}(p)$, logo, $q=p \backslash\left\{s \in 2^{<\omega}\right.$ : $f \mid(n+1) \subseteq s\} \in D$. Mas $G \cap D=\emptyset$, absurdo.

Para a terceira afirmação, suponha que $p \in G$. Dado $n \in \omega$, temos que $f \mid n \in p$, do contrário, teremos que $f \mid m \notin \bigcap G$ para todo $m \geq n$, o que viola o fato de que $\operatorname{dom} f=\omega$. Logo, $p \in\{q \in$ $\left.\mathbb{S}^{M}:\{f \mid n: n \in \omega\} \subseteq q\right\}$. Reciprocamente, suponha que $\{f \mid n: n \in \omega\} \subseteq p$ e suponha por absurdo que $p \notin \Gamma$. Então existe $q \in \mathbb{S}^{M}$ tal que: 


$$
q \Vdash \bigcap \Gamma \subseteq \check{p} \wedge \check{p} \notin \Gamma
$$

Afirmo que $q \leq p$. Pois assuma o contrário. Então existe $s \in q \backslash p$, e temos que $q \mid s \leq q$. Seja $H$ genérico tal que $q \mid s \in H$. Então temos que $s \in \bigcap H$, pois, caso contrário, como $q \mid s \in H$, segue que $\bigcap H$ é finito, o que viola o fato de que $\bigcup \bigcap H$ tem domínio $\omega$. Mas então, como $q \mid s \Vdash \bigcap H \subseteq \check{p}$, segue que $s \in p$, absurdo.

Assim, $q \leq p$. Mas então $q \Vdash \check{p} \in \Gamma$, o que é absurdo, pois $q \Vdash \check{p} \notin \Gamma$.

Definição 6.6. Seja $M$ uma interpretação transitiva e enumerável de uma teoria que estende ZFC. Dizemos que uma noção de forcing $P$ em $M$ adiciona reais se ${ }^{\omega} 2 \cap M \subsetneq{ }^{\omega} 2 \cap M[G]$ para todo $G$ $P$-genérico sobre $M$.

Notemos que o último lema nos diz que $\mathbb{S}^{M}$ adiciona reais.

Definição 6.7. Sejam $p, q \in \mathbb{S}$. Dizemos que $p \leq_{n} q$ se, e somente se, $p \leq q$ e $\operatorname{Br}(p)=\operatorname{Br}(q)$. Uma sequência de fusão é uma sequência $\left(p_{n}\right)_{n \in \omega}$ de elementos de $\mathbb{S}$ tal que $p_{n+1} \leq_{n+1} p_{n}$ para todo $n \in \omega$.

Se $A \subseteq p$ é uma coleção de pontos incompatíveis de $p$ e para cada $s \in S$, suponha que $p_{s} \in \mathbb{S}$ é tal que $p_{s} \leq p \mid s$, então a fusão de $\left(p_{s}: s \in A\right)$ em $p$ é o conjunto:

$$
\left\{t \in p: \forall s \in A\left(t \supset s \rightarrow t \in p_{s}\right)\right\}
$$

Ou seja, substitui-se os pontos acima de $s$ pelos de $p_{s}$.

Lema 6.15. Se $\left(p_{n}\right)_{n \in \omega}$ é uma sequência de fusão, então $p=\bigcap_{n \in \omega} p_{n} \in \mathbb{S}$.

Demonstração. Suponha que $s \in p \cap 2^{n}$. Temos que $s \in p_{n+1}$. Temos que existe $x \in \operatorname{Br}_{n+1}\left(p_{n+1}\right)$ com $s \supset x$. Temos que $x \in \mathrm{Br}_{n+1}\left(p_{m}\right)$ para todo $M, \operatorname{logo}, x \in \mathrm{Br}_{n+1}(p)$. Como $x$ é arbitrário, segue a tese.

Lema 6.16. Seja $p \in \mathbb{S}$ e seja $A \subseteq p$ uma coleção de pontos incompatíveis de $p$. Para cada $s \in S$, suponha que $p_{s} \in \mathbb{S}$ é tal que $p_{s} \leq p \mid s$. Então a fusão $q$ de $\left(p_{s}: s \in A\right)$ em $p$ é uma árvore perfeita.

Demonstração. Suponha primeiramente que não existe $s \in S$ tal que $q \in p_{s}$. Nesse caso, $s \mid p \subseteq q$, logo, existem dois elementos incompatíveis acima de $s$ em $q$. Caso contrário, existe $s \in S$ tal que $s \in p_{s}$, e existem dois elementos incompatíveis acima de $s$ em $p_{s} \subseteq q$.

Como uma aplicação básica das sequências de fusão, provaremos que o forcing de Sacks preserva $\omega_{1}$.

Proposição 6.17. Seja $M$ uma interpretação transitiva de ZFC e $G$ um $\mathbb{S}^{M}$ genérico sobre $M$. Então se $X \in M[G]$ é um conjunto enumerável (em $M[G]$ ) de ordinais, existe $A \in M$ conjunto de ordinais enumerável em $M$ tal que $X \subseteq A$.

Demonstração. Em $M[G]$, seja $f: \omega \rightarrow X$ bijetora. Seja $\alpha \in M$ ordinal tal que $X \subseteq \alpha$. Seja $\tau$ um nome tal que $\tau_{G}=f$. Suponha que a tese seja falsa e seja $K=[\alpha]^{\leq \omega} \cap M$. Existe $p \mathbb{S}$-nome tal que $p \Vdash \tau$ é uma função em $\check{\alpha}$ e $\forall x \in \check{K}(\tau[\check{\omega}] \nsubseteq x)$.

Agora argumentemos em $M$. Seja $p_{0}=p$. Definido $p_{n}$, para cada $s \in \operatorname{Br}_{n+1}\left(p_{n}\right)$ e para cada $i \in\{0,1\}$, sejam $t_{s \frown(i)} \leq p_{n} \mid s^{\frown}(i), \alpha_{s \frown(i)} \in \alpha$ tais que:

$$
t_{s \frown(i)} \Vdash^{*} \tau(n)=\check{\alpha}_{s} \frown(i) .
$$

Considere $p_{n+1}$ a fusão de $\left(t_{s \frown(i)}: s \in \operatorname{Br}\left(p_{n}\right), i \in\{0,1\}\right)$ em $p_{n}$. Seja $q=\bigcap_{n \in \omega} p_{n}$ e $A=$ $\left\{\alpha_{s \frown(i)}: \operatorname{Br}\left(p_{n}\right), i \in\{0,1\}, n \in \omega\right\}$

Voltemos a argumentar em $\mathbf{V}$. Temos que $q \Vdash \tau[\check{\omega}] \subseteq \check{A}$. Pois suponha que não. Então existe $u \leq q$ e $n \in \omega$ tal que $q \Vdash \tau(\check{n}) \notin \check{A}$, mas então para algum existe $s \in \operatorname{Br}(p)$ e $i \in 0,1$ tal que $s \frown(i) \in u$, e segue que

$$
u \mid s \frown(i) \Vdash^{*} \tau(n)=\check{\alpha}_{s \frown(i)} .
$$


Seja $H$ genérico tal que $q \in H$. Seja $h=\tau_{H}$. Temos que $h[\omega] \subseteq A$, pois $q \in A$, porém $h[\omega] \nsubseteq A$, pois $A \in K$ e $p=p_{0} \in H$. Absurdo.

Corolário 6.18. Seja $M$ uma interpretação transitiva de ZFC e $G$ um $\mathbb{S}^{M}$ genérico sobre $M$. Então $\omega_{1}^{M}=\omega_{1}^{M[G]}$

Demonstração. Suponha por absurdo que $\omega_{1}^{M}<\omega_{1}^{M[G]}$. Então $\omega_{1}^{M}$ é enumerável em $M[G]$, logo, pela proposição anterior, existe $A \in M$ enumerável em $M$ tal que $\left(\omega_{1} \subseteq A\right)^{M}$, o que implica que $\omega_{1}^{M}$ é enumerável em $M$, absurdo.

Lema 6.19. Seja $p$ árvore perfeita não vazia e suponha que $f: \mathbb{Q} \rightarrow \mathbb{Q}_{p}$ é isomorfismo de ordem. Então dado $A \subseteq \mathbb{Q}_{p}$, temos que existe $\phi: \operatorname{cl} f^{-1}[A] \rightarrow \operatorname{cl} A$ injetora tal que $\phi\left|f^{-1}[A]=f\right| f^{-1}[A]$. Em particular, temos que se $\operatorname{cl} f^{-1}[A]$ é não enumerável, então $\operatorname{cl} A$ é não enumerável.

Demonstração. Seja $B=\phi^{-1}[A]$. Se $x \in B$, define-se $\phi(x)=f(x)$. Agora suponha que $x \in \operatorname{cl} B \backslash B$. Para cada $n$ natural, seja $x_{n} \in B$ tal que $x_{n}|n=x| n$. Afirmo que a sequência $\phi\left(x_{n}\right) \in A$ converge. Como $\phi$ é isomorfismo de ordem, temos que dado $n, \phi\left(q\left(x_{n} \mid n\right)\right) \leq \phi\left(x_{n}\right)$, e a sequência $\phi_{n}\left(q\left(x_{n} \mid n\right)\right)$ converge, logo, a sequência $\phi\left(x_{n}\right)$ converge. Definamos $\phi(x)=\lim \phi\left(x_{n}\right)$. Notemos que se $x \in A$, podemos tomar $x_{n}=x$ para todo $n$ e adotar a mesma definição para ambos os casos.

Vejamos que $\phi$ é injetora. Suponha que $x \neq y$ são elementos de $B$. Existe $n$ tal que $x(n) \neq$ $x(n)$, então para todos $m, m^{\prime}>n$ temos que $x_{m}, y_{m^{\prime}}$ são incompatíveis. Assim, $\phi\left(x_{m}\right), \phi\left(y_{m}^{\prime}\right)$ são incompatíveis para todos $m, m^{\prime}>n$. o que mostra que as duas sequências convergem para pontos diferentes.

Proposição 6.20. Seja, $M$ uma interpretação transitiva e enumerável de ZFC e $\mathcal{I} \in M$ um ideal livre alto (em $M)$ em $\omega$. São equivalentes:

1. $\mathcal{I}$ é $\mathbb{S}^{M}$-indestrutível.

2. I é $P$-indestrutível para alguma noção de forcing $P \in M$ que adiciona reais.

3. $\left(\forall f: \mathbb{Q} \rightarrow \omega \exists I \in \mathcal{I}\left(\operatorname{cl} f^{-1}[I] \text { é não enumerável }\right)\right)^{M}$.

Demonstração. 1. Implica 2. trivialmente. Provaremos 2. implica 3. pela contrapositiva. Então suponha que existe $f: \mathbb{Q} \rightarrow \omega$ tal que para todo $I \in \mathcal{I}, \operatorname{cl}^{M} f^{-1}[I]$ é enumerável em $M$. Então, para todo $I \in \mathcal{I}, \mathrm{cl}^{-1}[I]=\mathrm{cl}^{M} f^{-1}[I]$; Veremos que se $P$ é uma noção de forcing em $M$ que adiciona um real, então $I$ é $P$-destrutível. Tome $G P$-genérico sobre $M$ e seja $r \in M[G] \cap 2^{\omega} \backslash M$ um novo real. Temos que $r \in 2^{\omega} \backslash \bigcup_{I \in \mathcal{I}} \operatorname{cl} f^{-1}[I]$. Seja $A=f\left[\left\{q_{r \mid n}: n \in \omega\right\}\right]$. Temos que $r \in \operatorname{cl} f^{-1}[A]$, então $A$ é infinito. Por absolutidade, $A \in M[G]$. Suponha por absurdo que existe $I \in \mathcal{I}$ tal que $I \cap A$ é infinito. Temos que $q_{r \mid n}$ está em $f^{-1}[I]$ para infinitos $n \in \omega$, o que implica que $r \in \operatorname{cl} f^{-1}[I]$, absurdo.

Agora veremos que 3. implica 1. Suponha que $\mathcal{I}$ satisfaça 3 mas não satisfaça 1. Então existe $p \in \mathbb{S}^{M}$ e um nome $\tau$ tal que $p \Vdash \tau$ é um subconjunto infinito de $\check{\omega} \wedge \forall x \in \check{\mathcal{I}}(x \cap \tau$ é finito). Agora, trabalhando em $M$, construiremos uma sequência de fusão. Seja $p_{0}=p$. Definido $p_{n}$, para cada $s \in \operatorname{Br}_{n+1}\left(p_{n}\right)$ e $i \in\{0,1\}$, seja $t_{s \frown(i)} \leq p_{n} \mid s \frown(i), a_{s} \in[\omega]^{<\omega}$ tal que:

$$
t_{s \frown(i)} \Vdash^{*} \tau \cap(\check{n}+\check{1})=\check{a}_{s} .
$$

Considere $p_{n+1}$ a fusão de $\left(t_{s \frown(i)}: s \in \mathrm{Br}_{n+1}\left(p_{n}\right), i \in\{0,1\}\right)$ em $p_{n}$. Seja $p^{\prime}=\bigcap_{n \in \omega} p_{n}$. Notemos que se $s \in \mathrm{Br}_{n+1}\left(p^{\prime}\right)$, então sendo $s^{\prime} \in \operatorname{Br}_{n}\left(p^{\prime}\right)$ com $s^{\prime} \subseteq s$, temos que existe $i$ tal que $p^{\prime}\left|s \leq p_{n}\right| t_{s^{\prime} \frown(i)}$,

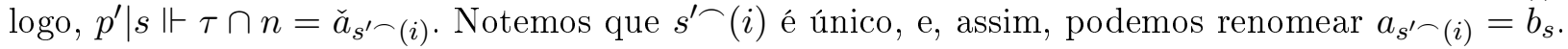
Assim, dado $s \in \operatorname{Br}_{n+1}\left(p^{\prime}\right)$ temos que existe $b_{s} \subseteq n$ tal que $p^{\prime} \mid s \Vdash^{*} \tau \cap n=\check{b}_{s}$.

Seja $f: \mathbb{Q}_{p^{\prime}} \rightarrow \omega$ dado por $f(q)=\max \left(\{0\} \cup\left\{k: p^{\prime} \mid s_{q} \Vdash \check{k} \in \tau\right\}\right)$. Verifiquemos que $f$ está bem definida. Dado $q$, suponha por absurdo que $\left\{k: p^{\prime} \mid s_{q} \Vdash \check{k} \in \tau\right\}$ é infinito. Então existe $I \in \mathcal{I}$ tal 
que $I \cap\left\{k: p^{\prime} \mid s_{q} \Vdash \check{k} \in \tau\right\}$ é infinito. Seja $H$ um filtro $\mathbb{S}^{M}$ genérico tal que $p^{\prime} \mid s_{q} \in H$. Temos que $p^{\prime} \mid s_{q} \leq p^{\prime} \leq p$, são elementos de $H$, então em $M[H]$ vale que $\left\{k: p^{\prime} \mid s_{q} \Vdash \check{k} \in \tau\right\} \cap I \subseteq \tau_{H}$, porém $I \cap \tau_{H}$ é finito. Absurdo.

Agora vamos trabalhar em $M$.

Afirmo que dado $k$ natural, $\mathrm{cl} f^{-1}[\{k\}]$ é enumerável. Pois suponha que não. Então existe $p^{\prime \prime} \in$ $\mathbb{S}^{M}$ tal que $p^{\prime \prime} \leq p^{\prime}$ e $\left[p^{\prime \prime}\right] \subseteq \operatorname{cl} f^{-1}[\{k\}]$. Como $p^{\prime \prime} \leq p$, então $p^{\prime \prime} \Vdash^{*} \exists m \in \check{\omega}(\tau \backslash(k \check{+} 1))$. Logo, existe $\tilde{p} \leq p^{\prime \prime}$ e $m \in \omega$ tal que $\tilde{p} \Vdash^{*} \check{m} \in \tau \backslash(k+1)$. Tomando $s \in \operatorname{Br}_{m+2}(\tilde{p})$, existe $l \geq m+2$ tal que $s \in \operatorname{Br}_{l+1}\left(p^{\prime}\right)$. Temos que $p^{\prime} \mid s \Vdash^{*} \tau \cap l=\check{b}_{s}$. Tomando um genérico, sai que $m \in b_{s}$. Seja $x \in[\tilde{p}] \backslash \mathbb{Q}$ tal que $s \subseteq x$. Seja $d$ tal que $x \mid d \backslash s$ assume o valor 1 duas vezes. Tomemos $t \in f^{-1}[\{k\}]$ tal que $t \supset x \mid d$. Temos que $s \subseteq s_{t}, \operatorname{logo}, p^{\prime}\left|s_{t} \leq p^{\prime}\right| s$. Por construção, $p^{\prime}\left|s_{t}\right| \nvdash^{*} \check{m} \in \tau$. Logo, existe $p^{*} \leq p^{\prime} \mid s_{t}$ tal que $p^{*} \Vdash^{*} \check{m} \notin \tau$, e, assim, $p^{*} \Vdash^{*} \check{m} \notin \check{b}_{s}$, mas isso implica que $m \notin b_{s}$, absurdo.

Seja $g: \mathbb{Q} \rightarrow \mathbb{Q}_{p^{\prime}}$ isomorfismo de ordem. Por 3., existe $I \in \mathcal{I}$ tal que $\operatorname{cl} g \circ f^{-1}[I]$ é não enumerável, logo, $\mathrm{cl}^{-1}[I]$ é não enumerável. Pelo parágrafo acima, temos que $I$ é infinito. Então existe $p^{\prime \prime} \in \mathbb{S}$ tal que $p^{\prime \prime} \leq p^{\prime},\left[p^{\prime \prime}\right] \subseteq \mathrm{cl}^{M} f^{-1}[I]$ e tal que para todo $x \in[p]$ e $n \in \omega$ existe $n^{\prime} \in \omega$ tal que $x|n \subseteq x| n^{\prime} \in p \cap f^{-^{\prime}}[I]$. Afirmo que $\left.p^{\prime \prime}\right|^{*}|\tau \cap \check{I}|=\check{\omega}$, o que gera um absurdo. Pois suponha que não.

Então existe $\tilde{p} \leq p^{\prime \prime}$ e $k \in \omega \backslash\{0\}$ tal que $\tilde{p} \Vdash^{*} \tau \cap \check{I} \subseteq \check{k}$. Seja $x \in[\tilde{p}] \backslash \bigcup_{n \in \omega} \operatorname{cl} f^{-1}[\{n\}]$. Então existe $m \in \omega$ tal que $x \mid m \in \operatorname{Br}(\tilde{p})$ e tal que $f\left(q_{x \mid m}\right) \geq k$, caso contrário teremos que $x \in \mathrm{cl} f^{-1}[k]$. Existe $t \in f^{-1}[I]$ tal que $x \mid m \subseteq s_{q}$ e $x \mid m \subseteq s_{t} \tilde{p}$. Seja $l=f(q)$. Como $x \mid m \subseteq s_{t}$, segue que $\left\{k \in \omega: p^{\prime}|x| m \Vdash^{*} \breve{k} \in \tau\right\} \subseteq\left\{k \in \omega: s_{t} \Vdash^{*} \breve{k} \in \tau\right\}$, e, portanto, $f(q(t)) \geq f\left(q_{x \mid m}\right)$. Segue que $p^{\prime} \mid q(t) \Vdash^{*} l \in \tau$, e, portanto, $\tilde{p} \mid q(t) \Vdash^{*} l \in \tau$, o que é absurdo.

Antes de provarmos o próximo teorema, provemos o seguinte lema:

Lema 6.21. $2^{\omega}$ pode ser escrito como uma união disjunta de $\mathfrak{c}$ subconjuntos perfeitos.

Demonstração. Sabemos que existe $f: 2^{\omega} \times 2^{\omega} \rightarrow 2^{\omega}$ homeomorfismo. Para cada $x \in 2^{\omega}$, o conjunto $P_{x}=\{x\} \times 2^{\omega}$ é perfeito e temos que $\bigcup_{x \in 2^{\omega}} P_{x}=2^{\omega}$.

Provaremos uma versão do Lema de König:

Lema 6.22 (König). Suponha que $p \subseteq 2^{<\omega}$ é uma árvore infinita. Então $[p] \neq \emptyset$.

Demonstração. Seja $s_{0}=\emptyset$. Definido $s_{m}$ para $m<n$ de modo que $s_{0} \subseteq s_{1} \ldots, s_{n-1}$, tal que $\left\{s \in p: s_{n-1} \subseteq s\right\}$ e tal que para cada $m<n$, o domínio de $s_{m}$ é $m$, seja $s_{n} \in 2^{n} \cap p$ tal que $s_{n} \supset s_{n-1}$ e tal $\left\{s \in p: s_{n} \subseteq s\right\}$ é infinito. Considere $\bigcup_{n \in \omega} s_{n}$.

Teorema 6.23. Em ZFC, toda MAD family de cardinalidade $<\mathfrak{c}$ é $\mathbb{S}$-indestrutível. Além disso, existe $\mathcal{A}$ MAD family $\mathbb{S}$-indestrutível.

Demonstração. Para a primeira afirmação, suponha que $\mathcal{A}$ é uma MAD family de cardinalidade $\kappa<\mathfrak{c}$. Se ela não for indestrutível, existe $f: \mathbb{Q} \rightarrow \omega$ tal que para todo $I \in \mathcal{I}(\mathcal{A})$, cl $f^{-1}[I]$ é enumerável. Tomemos $r \in 2^{\omega} \backslash \bigcup\left\{\operatorname{cl} f^{-1}[A]: A \in \mathcal{I}(\mathcal{A})\right\}$. Tome $B=f[\{q(r \mid n): n \in \omega\}]$. Como $r \in \mathrm{cl}^{-1}[B]$, temos que $B$ é finito e subconjuntos finitos de $\omega$ são elementos de $\mathcal{I}(\mathcal{A})$. Se $A \in \mathcal{I}(\mathcal{A})$, devemos ter que $A \cap B$ é finito, caso contrário teremos que $r \in \operatorname{cl}^{-1}[A]$. Mas isso viola que $\mathcal{A}$ é MAD.

Para a segunda afirmação, podemos supor, pela primeira afirmação, que $\mathfrak{a}=\mathfrak{c}$. Enumeremos todas as funções de $\mathbb{Q}$ em $\omega$ por $\left\{f_{\alpha}: \omega \leq \alpha<\mathfrak{c}\right\}$. Recursivamente, construiremos uma sequência de subconjuntos infinitos de omega $\mathcal{A}=\left\{A_{\alpha}: \alpha<\mathfrak{c}\right\}$ com as seguintes propriedades:

a) $\left\{A_{i}: i \in \omega\right\}$ é uma partição infinita de $\omega$

b) $\left|A_{\beta} \cap A_{\alpha}\right|<\omega$ sempre que $\beta<\alpha<\omega_{1}$.

c) $\exists \beta<\alpha\left(\operatorname{cl~} f_{\alpha}^{-1}\left[A_{\beta}\right]\right.$ é não enumerável) para todo $\beta<\mathfrak{c} \operatorname{com} \beta \geq \omega$. 
Suponha que é possível obter uma tal família. Então $\mathcal{A}$ é MAD, caso contrário, seja $B \subseteq \omega$ infinito tal que $\left|B \cap A_{\alpha}\right|<\omega$ para todo $\alpha<\mathfrak{c}$, e seja $f: \mathbb{Q} \rightarrow B$ bijetora. Existe $\alpha<\mathfrak{c}$ infinito tal que $f=f_{\alpha}$. Temos que existe $\beta<\alpha$ tal que $\operatorname{cl} f_{\alpha}^{-1}\left[A_{\beta}\right]=\operatorname{cl} f_{\alpha}^{-1}\left[A_{\beta} \cap B\right]$ é não enumerável, logo, $A_{\beta} \cap B$ é infinito já que $f_{\alpha}$ é injetora. Assim, segue a tese.

Devemos ver que é possível construir uma MAD family que satisfaz essas propriedades. Começamos fixando uma partição de $\omega$ infinita por subconjuntos infinitos $\left\{A_{i}: i \in \omega\right\}$. Suponha que $A_{\beta}$ foi construído para todo $\beta<\alpha$. Se existir $\gamma<\alpha$ tal que $\operatorname{cl~} f_{\alpha}\left[A_{\gamma}\right]$ é não enumerável, basta tomar $A_{\alpha}$ qualquer subconjunto infinito de $\omega$ almost disjoint de todos os anteriores (isso pode ser feito já que estamos supondo $\mathfrak{a}=\mathfrak{c})$.

Assim, suponha que não existe um tal $\gamma$. Seja $X=\bigcup\left\{\operatorname{cl} f_{\alpha}\left[A_{\beta}\right]: \beta<\alpha\right\}$. Temos que $|X|<\mathfrak{c}$. Devemos ter que, para todo natural $n, \operatorname{cl} f_{\alpha}^{-1}[\{n\}]$ é enumerável, ou teríamos que, para algum $i<\omega$, cl $f_{\alpha}^{-1}\left[A_{i}\right]$ é não enumerável. Seja $P \subseteq 2^{\omega}$ perfeito tal que $P \cap X=\emptyset$ (o que é possível, pois $2^{\omega}$ pode ser decomposto em $\mathfrak{c}$ subconjuntos perfeitos). Seja $A_{\alpha}=f_{\alpha}\left[\mathbb{Q}_{P}\right]$, onde $\mathbb{Q}_{P}=\mathbb{Q}_{p}$ para algum $p \in \mathbb{S}$ tal que $[p]=P$. Como cl $f^{-1}[F]$ é enumerável para todo $F \subseteq \omega$ finito, temos que $f_{\alpha}\left[\mathbb{Q}_{P}\right]$ é infinito, pois $P \subseteq \operatorname{cl} f_{\alpha}^{-1}\left[A_{\alpha}\right]$. Resta apenas ver que se $\beta<\alpha$ então $A_{\beta} \cap A_{\alpha}$ é finito. Assuma que não. Então existe uma sequência $\left\{s_{i}: i \in \omega\right\}$ tal que $s_{i} \in \operatorname{Br}(p)$ tal que se $i \neq j$ então $f\left(q\left(s_{i}\right)\right) \neq f\left(q\left(s_{j}\right)\right)$ e para todo $i, f_{\alpha}\left(q\left(s_{i}\right)\right) \in A_{\alpha} \cap A_{\beta}$. Tomando $g$ dada pela reunião de uma cadeia infinita na árvore $\left\{s_{i} \mid n: n, i \in \omega\right\}$, temos que $g \in P \cap \operatorname{cl} f_{\alpha}^{-1}\left[A_{\beta}\right]$ já que $g \in \operatorname{cl}\left\{q\left(s_{i}\right): i \in \omega\right\}$ (dado $n \in \omega$, por construção existe $i$ tal que $\left.s_{i}|n=g| n, \operatorname{logo}, q\left(s_{i}\right)|n=g| n\right)$. 


\section{Capítulo 7}

\section{Pseudocompacidade de hiperespaços}

\subsection{Introdução}

Neste capítulo, introduziremos a noção do hiperespaço (de Vietoris) associado a um espaço topológico arbitrário e em seguida provaremos algumas propriedades básicas, tomando como base o artigo [Mic51]. Depois disso, demonstraremos alguns resultados do artigo [HHR07] acerca de hiperespaços, incluindo construções envolvendo MAD families. Diversas topologias podem ser colocadas sobre a coleção dos fechados de um espaço topológico, e uma das construções mais antigas é a do hiperespaço de Vietoris, denotado por $\mathrm{CL}(X)$. Veremos que $\mathrm{CL}(X)$ é compacto se, e somente se $X$ é compacto, o que naturalmente induz a pergunta sobre se esta relação se generaliza de alguma forma para outras noções parecidas com compacidade. Em particular, estudaremos sobre se existe alguma relação entre a compacidade de $X^{\omega}$ e a de $\mathrm{CL}(X)$, questão colocada por J. Ginsburg em [Gin75].

Começaremos introduzindo a definição da topologia de Vietóris e provando alguns fatos básicos sobre estes hiperespaços.

Definição 7.1. Seja $X$ um espaço topológico. Dados $U, V_{0}, \ldots, V_{n-1} \subseteq X$, define-se:

$$
\left\langle U, V_{0}, \ldots, V_{n-1}\right\rangle=\left\{F \in \mathrm{CL}(X): F \subseteq U \wedge \forall i<n\left(F \cap V_{i} \neq \emptyset\right)\right\} .
$$

O hiperespaço (de Vietoris) de $X$ é o conjunto $C L(X)$ de todos os subconjuntos fechados não vazios de $X$ munido da topologia gerada pelos conjuntos da forma $\left\langle U, V_{0}, \ldots, V_{n-1}\right\rangle$ para $U$, $V_{0}, \ldots, V_{n}$ subconjuntos abertos de $X$.

Tal topologia é chamada de topologia de Vietoris.

Naturalmente, é interessante e útil verificar que os conjuntos acima formam uma base para a topologia gerada por eles. Tal fato é expresso pelo lema a seguir.

Lema 7.1. A coleção acima é uma base para uma topologia.

Demonstração. Temos que $\operatorname{CL}(X)=\langle X\rangle \mathrm{e}$

$$
\left\langle U, V_{0}, \ldots, V_{n-1}\right\rangle \cap\left\langle U^{\prime}, V_{0}^{\prime} \ldots, V_{m-1}^{\prime}\right\rangle=\left\langle U \cap U^{\prime}, V_{0}, \ldots, V_{n-1}, V_{0}^{\prime}, \ldots, V_{m-1}^{\prime}\right\} .
$$

Uma outra apresentação da topologia de Vietoris comum de ser encontrada na literatura é uma apresentação envolvendo a base abaixo. Por questão de completude, verificaremos a equivalência entre as duas.

Lema 7.2. Seja $X$ um espaço topológico. Então os conjuntos da forma $\{F \in \mathrm{CL}(X): F \subseteq$ $\left.\bigcup_{i<n} A_{i} \wedge \forall i<n\left(F \cap A_{i} \neq \emptyset\right)\right\}=\left\langle\bigcup_{i<n} A_{i}, A_{0}, \ldots, A_{n-1}\right\rangle$ para $A_{0}, \ldots, A_{n-1} \subseteq X$ abertos formam uma base para o espaço $\mathrm{CL}(X)$. 
Demonstração. Pela definição da topologia de Vietoris, cada um destes conjuntos é aberto. Reciprocamente, veremos que todo aberto básico pode ser escrito nessa forma. Dados $U, U_{0}, \ldots, U_{n-1}$, note que:

$$
\left\langle U, U_{0}, \ldots, U_{n-1}\right\rangle=\left\langle U \cup \bigcup_{i<n}\left(U_{i} \cap U\right), U, U_{0} \cap U, \ldots, U_{n-1} \cap U\right\rangle .
$$

E, assim, segue a tese.

Se $X$ é $T_{1}$, então $X$ pode ser facilmente identificado dentro de $\operatorname{CL}(X)$, como pode-se ver na proposição abaixo.

Proposição 7.3. Suponha que $X$ é $T_{1}$. Então $f: X \rightarrow \mathrm{CL}(X)$ dado por $f(x)=\{x\}$ é um homeomorfismo na imagem. Além disso, se $X$ é Hausdorff, então a imagem de $f$ é fechada.

Demonstração. $f$ é claramente injetora. Dados $U, V_{0}, \ldots, V_{n} \subseteq X$ abertos, temos que $f^{-1}\left[\left\langle U, V_{0} \cap\right.\right.$ $\left.\left.\cdots \cap V_{n-1}\right\rangle\right]=U \cap V_{0} \cdots \cap V_{n-1}$, que é aberto, e, se $U \subseteq X$ é aberto, temos que $f[U]=f[X] \cap\langle U\rangle$.

Agora suponha que $X$ é Hausdorff. Seja $F \in \mathrm{CL}(X) \backslash f[X]$. Temos que existem $x, y \in F$ tais que $x \neq y$. Sejam $U, V$ abertos tais que $x \in U, y \in V$ e $U \cap V=\emptyset$. Temos que $F \in\langle X, U, V\rangle \subseteq$ $\mathrm{CL}(X) \backslash f[X]$.

Vamos provar algumas propriedades sobre os abertos básicos.

Proposição 7.4. Sejam $X$ um espaço topológico, $U, V, U_{0}, \ldots, U_{n}, V_{0}, \ldots, V_{m} \subseteq X$. Então:

a) Se $U \subseteq V$ e para todo $j \leq m$ existe $i \leq n$ tal que $U_{i} \subseteq V_{j}$, então $\left\langle U, U_{0}, \ldots, U_{n}\right\rangle \subseteq$ $\left\langle V, V_{0}, \ldots, V_{m}\right\rangle$.

b) $\operatorname{cl}\left\langle U, U_{0}, \ldots, U_{n}\right\rangle \subseteq\left\langle\operatorname{cl} U, \operatorname{cl} U_{0}, \ldots, \operatorname{cl} U_{n}\right\rangle$. Se para todo $i \leq n, U_{i} \subseteq U$ e $U_{i}$ é aberto, então vale a igualdade.

Demonstração. a) Dado $F \in\left\langle U, U_{0}, \ldots, U_{n}\right\rangle$, temos que $F \subseteq U \subseteq V$ e dado $j \leq m$ temos que existe $i$ tal que $U_{i} \subseteq V_{j}$. Então temos que $\emptyset \neq U_{i} \cap F \subseteq V_{j} \cap F$. Isso prova que $F \in\left\langle V, V_{0}, \ldots, V_{m}\right\rangle$, e, como $F$ é arbitrário, segue que $\left\langle U, U_{0}, \ldots, U_{n}\right\rangle \subseteq\left\langle V, V_{0}, \ldots, V_{m}\right\rangle$.

b) Suponha que $F \in \operatorname{cl}\left\langle U, U_{0}, \ldots, U_{n}\right\rangle$. Vejamos primeiramente que $F \subseteq \operatorname{cl} U$. Suponha que não. $F \in\langle X, X \backslash \operatorname{cl} U\rangle$, mas $\langle X, X \backslash \operatorname{cl} U\rangle \cap\left\langle U, U_{0}, \ldots, U_{n}\right\rangle=\emptyset$, o que é absurdo. Além disso, dado $i \leq n$, temos que $F \cap c l U_{i} \neq \emptyset$, caso contrário teremos que $F \in\left\langle X \backslash \operatorname{cl} U_{i}\right\rangle$, mas $\left\langle X \backslash \operatorname{cl} U_{i}\right\rangle \cap\left\langle U, U_{0}, \ldots, U_{n}\right\rangle=$ $\emptyset$, o que é absurdo.

Para a segunda afirmação, tomemos $F \in\left\langle\operatorname{cl} U, \operatorname{cl} U_{0}, \ldots, \operatorname{cl} U_{n}\right\rangle$. Seja $\left\langle G, G_{0}, \ldots, G_{m}\right\rangle$ uma vizinhança aberta de $F$ tal que $G_{i} \subseteq G$. Dado $i \leq n$, existe $x_{i} \in F$ tal que $x_{i} \in F \cap$ cl $U_{i}$, logo, existe $y_{i} \in$ $G \cap U_{i}$. Para cada $j \leq m$, seja $z_{j} \in F \cap G_{j}$. Como $G_{j}$ é vizinhança aberta de $z_{j} \in \operatorname{cl} U$, existe $w_{j} \in G$ tal que $w_{j} \in G_{j} \cap U$. Segue que $\left\{y_{0}, \ldots, y_{n}, w_{0}, \ldots, w_{m}\right\} \in\left\langle G, G_{0}, \ldots, G_{m}\right\rangle \cap\left\langle U, U_{0}, \ldots, U_{m}\right\rangle \neq \emptyset$. Pela arbitrariedade da vizinhança de $F$, segue que $F \in \operatorname{cl}\left\langle U, U_{0}, \ldots, U_{n}\right\rangle$.

Agora começaremos a verificar algumas propriedades acerca da validade dos axiomas de separação nos hiperespaços.

Proposição 7.5. CL $(X)$ é $T_{0}$ qualquer que seja o espaço topológico $X$ (mesmo que $X$ não seja $\left.T_{0}\right)$. Além disso, se $X$ é $T_{1}$, segue que $\mathrm{CL}(X)$ é $T_{1}$ (e a recíproca não é verdadeira).

Demonstração. Se $F, K \subseteq X$ são fechados não vazios distintos, sem perda de generalidade existe $x \in K \backslash F$. Segue que $K \in\langle X, X \backslash F\rangle$ porém $F \notin\langle X, X \backslash F\rangle$. Isso mostra que $\operatorname{CL}(X)$ é $T_{0}$.

Se $X$ é $T_{1}$, sejam $F, K \subseteq X$ fechados distintos não vazios. Sem perda de generalidade existe $x \in K \backslash F$. Temos que $K \in\langle X, X \backslash F\rangle, F \notin\langle X, X \backslash F\rangle, F \in\langle X \backslash\{x\}\rangle$ e $K \notin\langle X \backslash\{x\}\rangle$.

Para um contra exemplo para a recíproca, considere qualquer conjunto $X$ com mais do que um elemento munido da topologia tal que os únicos abertos são $X$ e $\emptyset$. 
Proposição 7.6. Seja $X$ um espaço $T_{1}$. Temos que $\mathrm{CL}(X)$ é Hausdorff se, e somente se, $X$ é regular.

Demonstração. Suponha que $X$ é regular. Tomemos $F, G \subseteq X$ fechados distintos não vazios. Devemos separar $F, G$ por abertos em $\operatorname{CL}(X)$. Como $F \neq G$, sem perda de generalidade temos que $F \nsubseteq G$, logo, fixe $x \in F$ tal que $x \notin G$. Sejam $U, V \subseteq X$ abertos tais que $G \subseteq U, x \in V$ e $U \cap V=\emptyset$. Temos que $F \in\langle X \backslash G, V\rangle, G \in\langle U\rangle$, e que $\langle X \backslash G, V\rangle \cap\langle U\rangle=\emptyset$, pois se $H \in\langle X \backslash G, V\rangle \cap\langle U\rangle$, temos que $U \cap V \neq \emptyset$, o que é absurdo.

Reciprocamente, suponha que $X$ não é regular. Seja $F \subseteq X$ fechado e $x \in X$ tal que $x \notin F$ que não podem ser separados por abertos. Suponha que $U, U_{0}, \ldots, U_{n-1}, V, V_{0}, \ldots, V_{m-1}$ abertos de $X$ tais que:

$$
\begin{gathered}
F \in\left\langle U, U_{0}, \ldots, U_{n-1}\right\rangle, \\
F \cup\{x\} \in\left\langle V, V_{0}, \ldots, V_{m-1}\right\rangle .
\end{gathered}
$$

Podemos supor que para todos $i<n$ e $j<m$, temos que $U_{i} \subseteq U, V_{j} \subseteq V$.

Veremos que a interseção destes abertos básicos é não vazia. Para cada $j<m$, seja $a_{j} \in U \cap V_{j}$, o que existe pois, caso $x \in V_{j}$, então $V_{j} \cap U \neq \emptyset$ já que $F \subseteq U$ e $F, x$ não podem ser separados por abertos, e, caso $x \notin V_{j}$, temos que $(F \cup\{x\}) \cap V_{j}=F \cap V_{j} \neq \emptyset$. Para $i<n$, tomemos $b_{i} \in F \cap U_{i}$. Segue que $\left\{a_{0}, \ldots, a_{m-1}, b_{0}, \ldots, b_{n-1}\right\}$ é um ponto da interseção.

Proposição 7.7. Seja $X$ um espaço $T_{1}$. São equivalentes:

a) $X$ é normal.

b) $\mathrm{CL}(X)$ é regular.

Demonstração. Suponha que $X$ é normal. Sejam $F \subseteq X$ fechado e $U, U_{0}, \ldots, U_{n}$ tais que $F \in$ $\left\langle U, U_{0}, \ldots, U_{n}\right\rangle$. Como $F$ é normal, existe $V$ aberto tal que $F \subseteq V \subseteq \operatorname{cl} V \subseteq U$, e abertos $V_{i}$ para $i \leq n$ tais que $F \cap V_{i} \neq \emptyset$ e $\operatorname{cl} V_{i} \subseteq U_{i}$. Segue que $F \in\left\langle V, V_{0}, \ldots, V_{n}\right\rangle \subseteq \operatorname{cl}\left\langle V, V_{0}, \ldots, V_{n}\right\rangle \subseteq$ $\left\langle\operatorname{cl} V, \operatorname{cl} V_{0}, \ldots, \operatorname{cl} V_{n}\right\rangle \subseteq\left\langle U, U_{0}, \ldots, U_{n}\right\rangle$, e, portanto, $\mathrm{CL}(X)$ é regular.

Agora suponha que $\mathrm{CL}(X)$ é regular. Sejam $F \subseteq X$ fechado e $U \subseteq X$ aberto tal que $F \subseteq U$. Temos que $F \in\langle U\rangle$. Pela regularidade de $\mathrm{CL}(X)$, existem $V, V_{0}, \ldots, V_{n}$ abertos com $V_{i} \subseteq V$ para cada $i \leq n$ tal que $F \in\left\langle V, V_{0}, \ldots, V_{n}\right\rangle \subseteq \operatorname{cl}\left\langle V, V_{0}, \ldots, V_{n}\right\rangle=\left\langle\operatorname{cl} V, \operatorname{cl} V_{0}, \ldots, \operatorname{cl} V_{n}\right\rangle \subseteq\langle U\rangle$. Temos que $F \subseteq V \subseteq \operatorname{cl} V \subseteq U$, o que, pela arbitrariedade, atesta a normalidade de $X$ (a última desigualdade é verdadeira, caso contrário, se $x \in U \backslash \operatorname{cl} V$, teríamos que $\{x\} \in\langle U\rangle \backslash\left\langle\operatorname{cl} V, \operatorname{cl} V_{0}, \ldots, \operatorname{cl} V_{n}\right\rangle$, o que é absurdo).

A compacidade de $\mathrm{CL}(X)$ está intimamente relacionada com a compacidade de $X$, como veremos a seguir. Antes, vamos lembrar e provar o enunciado do Teorema da de Alexander.

Teorema 7.8. Seja $X$ um espaço topológico e $\mathcal{B}$ uma sub base de $X$. Se toda cobertura aberta de $\mathcal{B}$ possui uma subcobertura finita, então $X$ é compacto.

Demonstração. Suponha que não. Seja $\mathcal{U}$ a coleção de todas as coberturas abertas sem subcobertura finita. Pelo Lema de Zorn, $\mathcal{U}$ possui um elemento maximal, que iremos denotar por $\mathcal{C}$. Temos que $\mathcal{C} \cap \mathcal{B}$ não recobre $X$, ou teríamos, por hipótese, que $\mathcal{B} \cap \mathcal{C}$, e, portanto, $\mathcal{C}$, possui uma subcobertura finita. Seja $x \in X \backslash \bigcup \mathcal{B} \cap \mathcal{C}$. Existe $C \in \mathcal{C}$ tal que $x \in C$, e existem $B_{0}, \ldots, B_{n} \in \mathcal{B} \operatorname{com} x \in B_{1} \cap \cdots \cap B_{n} \subseteq C$. Pela escolha de $x$, temos que para cada $i \leq n, B_{i} \notin \mathcal{C}$. Pela maximalidade de $\mathcal{C}$, para cada $i \leq n$ temos que $\mathcal{C} \cup\left\{B_{i}\right\}$ possui uma subcobertura finita $\mathcal{C}^{i} \cup\left\{B_{i}\right\}$, com $\mathcal{C}^{i} \subseteq \mathcal{C}$. Temos que $\bigcup_{i \leq n} \mathcal{C}_{i} \cup\left\{\bigcap_{i \leq n} B_{i}\right\}$ recobre $X$. Mas então $\bigcup_{i \leq n} \mathcal{C}_{i} \cup\{C\} \subseteq \mathcal{C}$ recobre $X$, o que é absurdo.

Proposição 7.9. Seja $X$ um espaço topológico. Então $X$ é compacto se, e somente se, CL $(X)$ é compacto. 
Demonstração. Primeiro, suponha que $\mathrm{CL}(X)$ é compacto. Seja $\left\{U_{\alpha}: \alpha \in I\right\}$ uma cobertura aberta de $X$. Então $\left\{\left\langle X, U_{\alpha}\right\rangle: \alpha \in I\right\}$ é uma cobertura aberta de $\operatorname{CL}(X)$, e, portanto, existe $I_{0} \subseteq I$ finito tal que $\left\{\left\langle X, U_{\alpha}\right\rangle: \alpha \in I_{0}\right\}$ é uma cobertura aberta de $\operatorname{CL}(X)$. Então $X=\bigcup_{\alpha \in I_{0}} U_{\alpha}$, caso contrário $X \backslash \bigcup_{\alpha \in I_{0}} U_{\alpha}$ não é coberto por $\left\{\left\langle X, U_{\alpha}\right\rangle: \alpha \in I_{0}\right\}$.

Reciprocamente, suponha que $X$ é compacto. Para cada $U \subseteq X$, seja $U^{+}=\langle U\rangle, U^{-}=\langle X, U\rangle$. Notemos que $\left\langle U^{+}, U^{-}: U \subseteq X\right.$ aberto $\}$ forma uma subbase de $\operatorname{CL}(X)$. Seja $\mathcal{B}$ uma cobertura aberta de abertos subbásicos arbitrária. Podemos escrever $\mathcal{B}=\mathcal{B}_{0} \cup \mathcal{B}_{1}$, onde $\mathcal{B}_{0}=\left\{A^{+}: A \in \mathcal{A}_{0}\right\}$, $\mathcal{B}_{1}=\left\{A^{-}: A \in \mathcal{A}_{1}\right\}$, e $\mathcal{A}_{0}, \mathcal{A}_{1}$ são coleções de abertos de $X$. Pelo teorema da sub base de Alexander, é suficiente provar que $\mathcal{B}$ admite subcobertura finita. Seja $K=X \backslash \bigcup \mathcal{A}_{1}$, que é fechado. Existe $A_{0} \in \mathcal{A}_{0}$ tal que $K \in A^{+}$, caso contrário, existiria $A \in \mathcal{A}_{1}$ com $K \in A^{-}$, mas isso implica que existe $A \in \mathcal{A}_{1}$ tal que $A \cap K \neq \emptyset$, o que viola a definição de $K$. Fixe $A_{0}$ como acima. Como $K \cup \bigcup \mathcal{A}_{1}=X$, temos que $A_{0} \cup \cup A_{1}=X$. Assim, temos que existe $A_{1}^{\prime} \subseteq A_{1}$ finito tal que $X=A_{0} \cup \bigcup A_{1}^{\prime}$. Afirmo que $\left\{A_{0}^{+}\right\} \cup\left\{A^{-}: A \in \mathcal{A}_{1}^{\prime}\right\}$ é cobertura. Com efeito, se $F \subseteq K$, temos que $F \subseteq A_{0}$, e, assim, $F \in A_{0}^{+}$. Caso contrário, $F \cap \bigcup A_{1}^{\prime} \neq \emptyset$, e, assim, existe $A \in \mathcal{A}_{1}^{\prime}$ tal que $A \cap F=\emptyset$, o que nos diz que $F \in A^{-}$.

À luz do teorema acima, é natural perguntar-se qual a relação entre $X$ e $\operatorname{CL}(X)$ com respeito a noções mais fracas de compacidade, como compacidade enumerável e pseudocompacidade. J. Ginsburg, em [Gin75], provou que se CL $(X)$ é pseudocompacto, então $X^{n}$ é pseudocompacto para todo $n \in \omega$. Assim, ele perguntou se existe alguma relação entre a pseudocompacidade de $X^{\omega}$ e a de $\mathrm{CL}(X)$. Se restringirmos o universo dos espaços topológicos ao universo dos $\Psi$-espaços, a pergunta torna-se simplificada. Pelo Corolário 1.59 , se $\mathcal{A}$ é uma almost disjoint family e $X=\Psi(\mathcal{A}$, temos que $X^{\omega}$ é pseudocompacto se, e somente se $\mathcal{A}$ é MAD. Temos que se $\mathcal{A}$ não é MAD, $X$ não é pseudocompacto, portanto, $\mathrm{CL}(X)$ não é pseudocompacto. Assim, a pergunta se torna se $\mathcal{A}$ é uma mad family, o que ocorre com $\mathrm{CL}(\Psi(\mathcal{A})$ ? Veremos que sob $\mathfrak{h}<\mathfrak{c}$

No nosso contexto, o lema abaixo será de grande utilidade para a próxima seção.

Lema 7.10. Seja $X$ um espaço topológico Tychonoff e $N \subseteq X$ subconjunto denso de pontos isolados. Então $[N]^{<\omega} \backslash\{\emptyset\}$ é um denso em $\operatorname{CL}(X)$ de pontos isolados.

Demonstração. Primeiro, verificarmeos a densidade. Suponha que $\left\langle U, V_{0}, \ldots, V_{n-1}\right\rangle$ é não vazio e fixe $F \in\left\langle U, V_{0}, \ldots, V_{n-1}\right\rangle$. Para cada $i<n$, existe $x_{i} \in F$ tal que $x_{i} \in V_{i}$. Temos que $\left\{x_{i}\right\}_{i<n} \in$ $\left\langle U, V_{0}, \ldots, V_{n-1}\right\rangle$.

Para ver que se $F \subseteq N$ é finito então $F$ é isolado, sendo $F=\left\{x_{1}, \ldots, x_{n}\right\}$, temos que $\{F\}=$ $\left\langle F,\left\{x_{1}\right\}, \ldots,\left\{x_{n}\right\}\right\rangle$.

\subsection{Limites por ultrafiltros}

Antes de prosseguirmos, relembraremos a noção de $p$ limite para um filtro $p$ e provaremos algumas de suas propriedades mais básicas.

Definição 7.2. Sejam $p \subseteq \mathscr{P}(\omega)$ um filtro, $X$ um espaço topológico, $\left(x_{n}: n \in \omega\right)$ uma sequência em $X$ e $a \in X$. Dizemos que $a$ é $p$-limite de $\left(x_{n}: n \in \omega\right)$ se, e somente se, para toda vizinhança $V$ de $a$, temos que $\left\{n \in \omega: x_{n} \in V\right\} \in p$.

Lema 7.11. Seja $p \subseteq \mathscr{P}(\omega)$ um filtro, $X$ um espaço topológico e $x=\left(x_{n}: n \in \omega\right)$ uma sequência em $X$.

i) Se $X$ é Hausdorff e $p$ é um filtro próprio, a sequência $x$ possui no máximo um $p$-limite, e denotamos-o por $p-\lim x$ ou $p-\lim x_{n}$.

ii) Se $p$ é o ultrafiltro fixo gerado por $n$, então $x_{n}$ é $p$-limite de $x$.

iii) Se a sequência $x$ converge para $a \in X$ e se $p$ é um filtro livre, então $a$ é $p$-limite de $x$.

iv) Se $X$ é compacto e $p$ é um ultrafiltro, existe um $p$-limite de $x$. 
Demonstração. $\quad$ i) Se $a, b$ forem dois $p$-limites distintos de $x$, então tomemos $U, V$ vizinhanças disjuntas desses. Temos que $\emptyset=\left\{n \in \omega: x_{n} \in U\right\} \cap\left\{n \in \omega: x_{n} \in V\right\} \in p$, o que é absurdo.

ii) Se $x_{n} \in V$, temos que $n \in\left\{m \in \omega: x_{m} \in V\right\}$, $\log \left\{m \in \omega: x_{m} \in V\right\} \in p$. Assim, segue a tese.

iii) Seja $V$ uma vizinhança aberta de $a$. Temos que $\left\{n \in \omega: x_{n} \in V\right\}$ é cofinito, logo, está em $p$.

iv) Suponha que não. Então para todo $a \in X$ existe $V_{a} \subseteq X$ aberto tal que $U_{a}=\left\{n \in \omega: x_{n} \in\right.$ $\left.V_{a}\right\} \notin p$. Como $X$ é compacto, existe $A \subseteq X$ finito tal que $\bigcup_{a \in A} V_{a}=X$. Logo, $\bigcup_{a \in A} U_{a}=\omega$. Mas então, como $p$ é ultrafiltro, existe $a \in A$ tal que $U_{a} \in p$, o que é absurdo.

Devido aos itens $i$ ) e $i i i)$ do lema acima, a maioria dos textos exige que $p$ seja um filtro livre, pois, dessa forma, o $p$-limite é uma extensão do limite de função usual. Ainda, devido a $i v$ ), a maioria das aplicações faz uso apenas de ultrafiltros livres. Nossas aplicações não serão diferentes, porém optamos por introduzir a noção de $p$-limite através dessa definição mais geral. Veremos a seguir que a noção de $p$-limite preserva diversas propriedades boas das diferentes noções de convergência presentes em topologia. Começaremos mostrando que os $p$-limites são "levados" através de funções contínuas.

Lema 7.12. Seja $f: X \rightarrow Y$ uma função contínua entre espaços topológicos $X, Y$. Seja $p$ um filtro em $\omega, x=\left(x_{n}: n \in \omega\right)$ uma sequência em $X$ e $a \in X$. Se $a$ é $p$-limite de $x$, então $f(a)$ é $p$-limite de $f \circ x$.

Demonstração. Seja $V$ uma vizinhança de $f(a)$. Devemos ver que $\left\{n \in \omega: f\left(x_{n}\right) \in V\right\} \in p$. Seja $U$ vizinhança de $a$ tal que $f[U] \subseteq V$. Temos que $\left\{n \in \omega: x_{n} \in U\right\} \subseteq\left\{n \in \omega: f\left(x_{n}\right) \in V\right\}$. Como o primeiro está em $p$, o segundo também está.

Também é verdade que o $p$-limite se comporta bem em produtos.

Lema 7.13. Seja $\left(X_{\alpha}: \alpha \in I\right)$ uma família de espaços topológicos e $X=\prod_{\alpha \in I} X_{\alpha}$. Seja $a=\left(a_{\alpha}\right.$ : $\alpha \in I) \in X$, e seja $x$ uma sequência em $X$. Seja $p$ um filtro sobre $\omega$. Então $a$ é $p$-limite de $x$ se, e somente se, para todo $\alpha \in I, a_{\alpha}$ é $p$-limite de $((x(n))(\alpha): n \in \omega)$.

Demonstração. Se $a$ é $p$-limite de $x$, como a $\alpha$-ésima projeção é contínua, segue do lema anterior que $a_{\alpha}$ é $p$-limite de $((x(n))(\alpha): n \in \omega)$. Reciprocamente, suponha que para todo $\alpha \in I, a_{\alpha}$ é $p$-limite de $((x(n))(\alpha): n \in \omega)$. Seja $U=\bigcap_{\alpha \in J} \pi_{\alpha}^{-1}\left[A_{\alpha}\right]$, uma vizinhança básica de $a$ em $X$ onde $A_{\alpha} \subseteq X_{\alpha}$ é uma vizinhança aberta de $x_{\alpha}$ para todo $\alpha \in J \subseteq I$, onde $J \subseteq I$ é finito. Para cada $\alpha \in J$, temos que $\left\{n \in \omega:(x(n))(\alpha) \in A_{\alpha}\right\} \in p$. Como $J$ é finito e $\bigcap_{\alpha \in J}\{n \in \omega:(x(n))(\alpha) \in$ $\left.A_{\alpha}\right\} \subseteq\{n \in \omega: x(n) \in U\}$, segue a tese.

O lema abaixo é extremamente útil no estudo de compacidade enumerável.

Lema 7.14. Sejam $X$ um espaço topológico e $A=\left\{x_{n}: n \in \omega\right\}$ a imagem de uma sequência injetora $x$ em $X$. Temos que $a \in X$ é $\omega$-ponto de acumulação de $A$ se, e somente se, existe um ultrafiltro livre $p$ tal que $a$ é $p$-limite de $x$ em $X$.

Demonstração. Suponha que $p$ seja um $\omega$-ponto de acumulação de $A$. Então para toda vizinhança $V$ de $a$, o conjunto $\tilde{V}=\left\{n \in \omega: x_{n} \in V\right\}$ é infinito. Seja $\mathcal{V}$ o filtro das vizinhanças de $x$. Como $\mathcal{V}$ é fechado para interseções finitas, temos que $\{\tilde{V}: V \in \mathcal{V}\}$ possui a pif forte, logo, está contido em um ultrafiltro livre $p$. Por construção, segue que a $a$ é $p$ limite de $x$.

Reciprocamente, se $a$ é $p$-limite de $x$, é claro que toda vizinhança de $a$ intersecta infinitos elementos de $\left\{x_{n}: n \in \omega\right\}$, já que $p$ é livre e $x$ injetora.

Com isso, obtemos o seguinte corolário: 
Corolário 7.15. Seja $X$ um espaço topológico infinito e seja $A \subseteq X$ denso. Se toda sequência em $A$ possui um $p$-limite em $X$ para algum ultrafiltro livre $p$, então $A$ é relativamente enumeravelmente compacto em $X$, e, portanto, $X$ é pseudocompacto.

\subsection{Pseudocompacidade de $\mathrm{CL}(\Psi(\mathcal{A}))$ sob $\mathfrak{p}=\mathfrak{c}$}

O objetivo desta seção é provar que admitindo-se $\mathfrak{p}=\mathfrak{c}$, que é equivalente ao Axioma de Martin para ordens parciais $\sigma$-centradas, segue que para toda MAD family $\mathcal{A}, \mathrm{CL}(\Psi(\mathcal{A}))$ é pseudocompacto.

Daremos duas demonstrações, uma clássica, encontrada no artigo [HHR07] e outra inédita, desenvolvida utilizando técnicas diferentes e que consideramos ser mais simples.

Começaremos pela demonstração clássica. Para esta parte dessa seção, utilizaremos as seguintes definições:

Definição 7.3. Sejam $\mathcal{A}$ uma almost disjoint family, $Y=\left\{F_{n}: n \in \omega\right\}$ a imagem de uma sequência injetora de subconjuntos finitos não vazios de $\omega, A \subseteq \omega$ e $F \in \operatorname{CL}(\Psi(\mathcal{A}))$. Define-se:

- $I_{A}=\left\{n \in \omega: F_{n} \cap A \neq \emptyset\right\}$

- $M_{A}=\left\{n \in \omega: F_{n} \subseteq A\right\}$

- $\mathcal{F}_{F}=\left\{I_{A \backslash k}: A \in F \cap \mathcal{A}, k \in \omega\right\} \cup\left\{I_{\{n\}}: n \in \omega \cap F\right\}$.

Proposição 7.16. Na notação acima, são equivalentes:

(1) $F$ é ponto de acumulação da sequência $Y \operatorname{em~} \operatorname{CL}(\Psi(\mathcal{A}))$.

(2) Para todo $P \subseteq \omega$, se $F \cap \omega \subseteq P$ e se para todo $A \in F \cap \mathcal{A}, A \subseteq \subseteq^{*} P$, então a família $\mathcal{F}_{F} \cup\left\{M_{P}\right\}$ possui a propriedade da interseção finita forte.

Demonstração. Suponha que $F$ é ponto de acumulação da sequência $Y$ em $\operatorname{CL}(\Psi(\mathcal{A}))$. Seja $P$ tal que $F \cap \omega \subseteq P$ e tal que para todo $A \in F \cap \mathcal{A}, A \subseteq \subseteq^{*} P$. Seja $V=P \cup(F \cap \mathcal{A})$. Temos que $V$ é aberto em $\Psi(\mathcal{A})$ e $F \subseteq V$. Seja

$$
Q=\left\{I_{A_{0} \backslash k_{0}}, \ldots, I_{A_{m} \backslash k_{m}}\right\} \cup\left\{I_{\left\{a_{0}\right\}} \ldots I_{\left\{a_{l}\right\}}\right\} \cup\left\{M_{P}\right\},
$$

onde $A_{i} \in F \cap \mathcal{A}, k_{i} \in \omega$ para todo $i \leq m$, e $a_{j} \in \omega \cap F$ para todo $j \leq l$. Seja

$$
U=\left\langle V,\left\{A_{0}\right\} \cup A_{0} \backslash k_{0}, \ldots,\left\{A_{m}\right\} \cup A_{m} \backslash k_{m},\left\{a_{0}\right\}, \ldots,\left\{a_{l}\right\}\right\rangle .
$$

Temos que $U$ é uma vizinhança de $F$ em $\mathrm{CL}(\Psi(\mathcal{A}))$ e, portanto, $Y \cap U$ é infinito. Assim, existe $I \subseteq \omega$ infinito tal que se $k \in I$, então $F_{k} \subseteq V, F_{k} \cap\left(A_{i} \backslash k_{i}\right) \neq \emptyset$ e $a_{j} \in F_{k}$ para $i \leq m$ e $j \leq l$. Note que como $F_{n} \subseteq \omega$, segue que $F_{n} \subseteq P$. Logo, pelas definições, $I \in \bigcap \mathcal{Q}$. Isso mostra que $\mathcal{F}_{F} \cup\left\{M_{P}\right\}$ possui a propriedade da interseção finita forte.

Reciprocamente, suponha (2). Veremos que vale (1). Um aberto básico ao qual $F$ pertence é da forma:

$$
U=\left\langle V,\left\{A_{0}\right\} \cup A_{0} \backslash k_{0}, \ldots,\left\{A_{m}\right\} \cup A_{m} \backslash k_{m},\left\{a_{0}\right\}, \ldots,\left\{a_{l}\right\}\right\rangle,
$$

$\operatorname{com} F \subseteq V, V$ aberto, $A_{0}, \ldots, A_{m} \in F \cap \mathcal{A}, k_{0}, \ldots, k_{n} \in \omega$ e $a_{0}, \ldots, a_{l} \in \omega \cap F$.

Seja $P=\omega \cap V$. Como $F \subseteq V$, segue que $F \cap \omega \subseteq P$. Além disso, se $A \in \mathcal{A} \cap F$, temos que, como $A \in V$, que é aberto, existe $k^{\prime}$ tal que $A \backslash k^{\prime} \subseteq V$, mas então $A \subseteq \subseteq^{*} P$. Logo, $\mathcal{F}_{F} \cup\left\{M_{P}\right\}$ possui a propriedade da interseção finita forte. Logo, a interseção do conjunto abaixo é infinita:

$$
\left\{I_{A_{0} \backslash k_{0}}, \ldots, I_{A_{m} \backslash k_{m}}\right\} \cup\left\{I_{\left\{a_{0}\right\}} \ldots I_{\left\{a_{l}\right\}}\right\} \cup\left\{M_{P}\right\}
$$

Seja $I$ a interseção. Temos que $\left\{F_{i}: i \in I\right\} \subseteq U \cap Y$ e segue a tese. 
Utilizaremos o seguinte lema:

Lema 7.17. Seja $X$ um espaço topológico $T_{1}$ e $D \subseteq X$ infinito discreto denso em $X$. São equivalentes:

a) $X$ é pseudocompacto

b) $D$ é relativamente enumeravelmente compacto em $X$.

Demonstração. Provaremos as duas implicações pela contrapositiva. Primeiramente, suponha que $X$ não é pseudocompacto e tome $f: X \rightarrow[0, \infty)$ contínua e ilimitada. Dado $n \in \omega$ existe $d_{n} \in D$ com $f\left(d_{n}\right)>n$. Suponha por absurdo que existe $x$ ponto de acumulação de $D^{\prime}=\left\{d_{n}: n \in \omega\right\}$. Dado $n \in \omega$, temos que $f^{-1}[[n, \omega)]$ é um fechado que contém uma coleção cofinita de elementos de $D^{\prime}$, e, portanto, $x$ está nela. Logo, $f(x) \geq n$. Como $n$ é arbitrário, obtemos um absurdo.

Reciprocamente, suponha que $D$ não é relativamente enumeravelmente compacto em $X$. Então existe $D^{\prime} \subseteq D$ infinito enumerável sem pontos de acumulação. Como $D^{\prime}$ é clopen, podemos tomar $f: D^{\prime} \rightarrow \omega$ bijetora e $g: X \backslash D^{\prime} \rightarrow \mathbb{R}$ constante. Segue que $f \cup g: X \rightarrow \mathbb{R}$ é contínua e ilimitada.

Teorema $7.18(\mathfrak{p}=\mathfrak{c}) \cdot \operatorname{CL}(\Psi(\mathcal{A}))$ é pseudocompacto para toda MAD family $\mathcal{A}$.

Demonstração. Temos que $\omega$ é um subconjunto de $\Psi(\mathcal{A})$ denso de pontos isolados. Assim, $J=$ $[\omega]^{<\omega} \backslash \emptyset \subseteq \mathrm{CL}(\Psi(\mathcal{A}))$ é um subconjunto denso de pontos isolados. Basta ver que $[\omega]^{<\omega} \backslash \emptyset$ é relativamente enumeravelmente compacto em $\mathrm{CL}(\Psi(\mathcal{A}))$.

Para isso, fixe $Y=\left\{F_{n}: n \in \omega\right\} \subseteq[\omega]^{<\omega} \backslash \emptyset$ imagem de uma sequência injetora em $[\omega]^{<\omega} \backslash$ $\emptyset$. Escrevamos $[\omega]^{\omega}=\left\{P_{\alpha}: \alpha<\mathfrak{c}\right\}$ de modo que cada elemento se repete $\mathfrak{c}$ vezes e $P_{0}=\omega$. Recursivamente, construiremos uma família $\left\{E_{\alpha}: \alpha<\mathfrak{c}\right\}$ tal que:

(1) $E_{\alpha} \subseteq \Psi(\mathcal{A})$

(2) $\left|E_{\alpha}\right| \leq|\alpha|+|\omega|$

(3) $\alpha \leq \beta \Longrightarrow E_{\alpha} \subseteq E_{\beta}$,

(4) $\mathcal{F}_{\alpha}=\left\{I_{A \backslash k}: A \in E_{\alpha} \cap \mathcal{A}, k \in \omega\right\} \cup\left\{I_{\{n\}}: n \in E_{\alpha} \cap \omega\right\}$ possui a propriedade da interseção finita forte,

(5) um dos seguintes ocorre:

(a) $\left(E_{\alpha} \cap \omega\right) \backslash P_{\alpha} \neq \emptyset$,

(b) Existe $A \in E_{\alpha} \cap \mathcal{A}$ tal que $\left|A \backslash P_{\alpha}\right|=\omega$,

(c) $\mathcal{F}_{\alpha} \cup\left\{M_{P_{\alpha}}\right\}$ possui a propriedade da interseção finita forte.

Mostraremos que uma tal construção é possível. Depois, mostraremos que o fecho da união da sequência construída é um ponto de acumulação de $Y$, o que completa a prova.

Temos que $\bigcup Y$ é infinito, logo, existe $A \in \mathcal{A}$ tal que $\bigcup Y \cap A$ é infinito. Note que, dado $k \in \omega$ existe $n \in \omega$ tal que $F_{n} \backslash k \cap A \neq \emptyset$. Seja $E_{0}=\{A\}$. Temos que (1) é satisfeito pois $E_{0}=\subseteq \mathcal{A},(2)$ é satisfeito pois $\left|E_{0}\right|=1$. (3) é trivialmente satisfeito até $\beta=0$. Para (4), o segundo conjunto que define $\mathcal{F}_{0}$ é vazio. Assim, basta ver que $\left\{I_{A \backslash k}: k \in \omega\right\}$ possui a propriedade da interseção finita forte. Sejam $k_{0}, \ldots, k_{l} \in \omega$, e seja $k$ o maior deles. Temos que $I_{A \backslash k_{0}} \cap \cdots \cap I_{A \backslash k_{n}}=I_{A \backslash k}$, e este último é infinito pela escolha de $A$. Como $M_{P_{\alpha}}=M_{\omega}$, segue que vale (5)(c).

Suponha que $0<\alpha<\mathfrak{c}$ e que $E_{\beta}$ foi construído para todo $\beta<\alpha$. Como $\mathcal{F}_{\beta}$ possui a pif forte para todo $\beta<\alpha$, então $\mathcal{F}=\bigcup_{\beta<\alpha} F_{\beta}$ também possui. Se $\mathcal{F} \cup\left\{M_{P_{\alpha}}\right\}$ possui a pif forte, seja $E_{\alpha} \bigcup_{\beta<\alpha} E_{\beta}$. Segue que $\mathcal{F}_{\alpha}=\mathcal{F}$ e todas as propriedades são satisfeitas. Caso contrário, seja $J$ uma pseudointerseção de $\mathcal{F}$. Devemos ter que $J \backslash P_{\alpha}$ é infinito, pois se $J \subseteq^{*} P_{\alpha}$, teremos que $\mathcal{F} \cup\left\{M_{P_{\alpha}}\right\}$ possui a pif forte. Logo, tomando $J \backslash P_{\alpha}$, podemos supor que $J \cap P_{\alpha}=\emptyset$. Agora dividimos a situação em dois casos. 
Caso 1: Existe $m \in \omega \backslash P_{\alpha}$ tal que $\left\{n \in J: m \in F_{n}\right\}$ é infinito.

Nesse caso, seja $E_{\alpha}=\bigcup_{\beta<\alpha} E_{\beta} \cup\{m\}$. Claramente, valem (1), (2), (3) e (5)(a). Veremos que vale (4). Fixemos $A_{0}, \ldots, A_{s} \in \mathcal{F}_{\alpha} \cap \mathcal{A}, k_{0}, \ldots, k_{s} \in \omega a_{0}, \ldots, a_{t} \in E_{\alpha} \cap \omega \backslash\{m\}$. Seja:

$$
\mathcal{G}=\left\{I_{A_{0} \backslash k_{0}}, \ldots, I_{A_{s} \backslash k_{s}}, I_{a_{0}}, \ldots, I_{a_{t}}, I_{m}\right\}
$$

Temos que $\mathcal{G} \subseteq \mathcal{F} \cup\left\{I_{\{m\}}\right\}$. Temos que $\left\{n \in J: m \in F_{n}\right\}=J \cap I_{m}$ é uma pseudointerseção de $\mathcal{F}$ contida em $I_{\{m\}}$, então segue a tese.

Caso 2: Para todo $m \in \omega \backslash P_{\alpha},\left\{n \in J: m \in F_{n}\right\}$ é finito.

Lembremos primeiramente que $M_{P_{\alpha}} \cap J=\emptyset$. Logo, se $n \in J$, temos que $F_{n} \notin M_{P_{\alpha}}$, ou seja, $F_{n} \nsubseteq P_{\alpha}$. Assim, dado $n \in J$, temos que existe $h(n) \in F_{n} \backslash P_{\alpha}$. Assim, define-se $h: J \rightarrow \bigcup_{n \in J} F_{n} \backslash P_{\alpha}$. Temos que $J=\bigcup_{x \in h[J]} h^{-1}[\{x\}]$. Dado $x \in h[J]$, temos que $h^{-1}[\{x\}]=\{n \in J: h(n)=x\} \subseteq\{n \in$ $\left.J: x \in F_{n}\right\}$, que é finito. Como $J$ é infinito, segue que $h[J]$ é infinito.

Assim, existe $A \in \mathcal{A}$ tal que $|A \cap h[J]|=\omega$. Seja $E_{\alpha}=\bigcup_{\beta<\alpha} E_{\beta} \cup\{A\}$. Seguem trivialmente (1), (2), (3) e (5) (b). Verificaremos (4).

Fixemos $A_{0}, \ldots, A_{s} \in E_{\alpha} \cap \mathcal{A} \backslash\{A\}, k, k_{0}, \ldots, k_{s} \in \omega a_{0}, \ldots, a_{t} \in E_{\alpha} \cap \omega$. Seja:

$$
\mathcal{G}=\left\{I_{A_{0} \backslash k_{0}}, \ldots, I_{A_{s} \backslash k_{s}}, I_{a_{0}}, \ldots, I_{a_{t}}, I_{A \backslash k}\right\} \subseteq \mathcal{F} \cup\left\{I_{A \backslash k}\right\}
$$

Seja $K=h^{-1}[A]=h^{-1}[A \cap h[J]]$, que é infinito pois $A \cap h[J]$ é infinito. Como $K \subseteq J$, temos que $K \subseteq^{*} F$ para todo $F \in \mathcal{F}$. Resta ver que $K \subseteq^{*} I_{A \backslash k}$. Suponha por absurdo que $K \backslash I_{A \backslash k}$ seja infinito. Isso implica que existem infinitos $n \in K$ para os quais $F_{n} \cap A \backslash k=\emptyset$. Como $\forall n \in K\left(h(n) \in A \cap F_{n}\right)$, existem infinitos $n$ tais que $h(n)<k$, o que implica que a imagem inversa por $h$ de algum conjunto unitário é infinita, o que é absurdo.

Isso completa a construção da sequência. Seja $E=\operatorname{cl} \bigcup_{\alpha<\mathfrak{c}} E_{\alpha}$. Veremos que $E$ é ponto de acumulação de $Y$. Para isso, basta provar que para todo $P \subseteq \omega$, uma das três condições abaixo deve valer:

(1) $(E \cap \omega) \backslash P \neq \emptyset$,

(2) Existe $A \in E \cap \mathcal{A}$ tal que $|A \backslash P|=\omega$, ou

(3) $\mathcal{F}_{E} \cup\left\{M_{P}\right\}$ possui a pif forte.

Primeiro, veremos que $\mathcal{F}_{E}$ possui a pif forte. Temos que $\mathcal{F}=\bigcup_{\alpha<\mathfrak{c}} E_{\alpha}$ claramente possui a pif forte. Além disso, $E \backslash \bigcup_{\alpha<\mathfrak{c}} E_{\alpha} \subseteq \mathcal{A}$ pois todo ponto de $\omega$ é isolado. Além disso, para todo $A$ nesta diferença e $k \in \omega$, temos que $(A \backslash k) \cap \bigcup_{\alpha<\mathfrak{c}} E_{\alpha} \neq \emptyset$ Tomando $m$ neste conjunto, temos que $I_{\{m\}} \subseteq I_{A \backslash k}$. Isso implica que se $F \in \mathcal{F}_{E}$ existe $G \in \mathcal{F}$ tal que $G \subseteq F$. Assim, como $\mathcal{F}$ possui a pif forte, $\mathcal{F}_{E}$ também possui.

Agora fixemos $P \subseteq \omega$ e suponhamos que $\mathcal{F}_{E} \cup\left\{M_{P}\right\}$ não possua a pif forte. Existem $A_{0}, \ldots, A_{n} \in$ $E \cap \mathcal{A}, k_{0}, \ldots, k_{n} \in \omega, m_{0}, \ldots, m_{k} \in E \cap \omega$ tais que

$$
\bigcap_{i \leq n} I_{A_{i} \backslash k_{i}} \cap \bigcap_{j \leq k} I_{\left\{m_{j}\right\}} \cap M_{P}
$$

é finito. Como vimos no parágrafo anterior, para cada $i \leq n$ tal que $A_{i} \in E \backslash \bigcup_{\alpha<\mathfrak{c}} E_{\alpha}$, existe $\alpha_{i}<\mathfrak{c}$ e $\alpha_{i}$ tal que $I_{m_{i}} \subseteq I_{A_{i} \backslash k_{i}}$. Tomemos $\beta<\mathfrak{c}$ maior do que todos os $\alpha_{i}$, tal que $A_{i} \in E_{\beta}$ para todo $i$ para o qual $A_{i} \in \bigcup_{\alpha<\mathfrak{c}} E_{\alpha}$ e tal que $m_{j} \in E_{\beta}$ para todo $j \leq k$. Seja $\alpha>\beta$ tal que $P=P_{\alpha}$. Segue que $\mathcal{F}_{\alpha} \cup\left\{M_{P_{\alpha}}\right\}$ também não tem a pif forte. Logo, existe $A \in E_{\alpha} \cap A \subseteq E \cap A$ tal que $\left|A \backslash P_{\alpha}\right|=|A \backslash P|=\omega$, ou $\emptyset \neq E_{\alpha} \cap \omega \backslash P_{\alpha} \subseteq(E \cap \omega) \backslash P$. Assim, segue a tese.

Agora daremos nossa demonstração, que consideramos ser mais simples que a demonstração já apresentada nessa seção. 
Definição 7.4. Dado um ordinal $\alpha$, uma $\supset^{*} \alpha$-torre, ou, simplesmente, uma $\alpha$-torre, é uma família de subconjuntos infinitos de $\omega\left(A_{\beta}: \beta<\alpha\right)$ tal que se $\gamma<\beta<\alpha$ então $A_{\gamma} \supset^{*} A_{\beta}$. $\mathfrak{t}$ é o menor ordinal tal que existe uma $\mathbf{t}$-torre sem uma pseudointerseção.

O lema a seguir é de fácil demonstração e está provado em [Bla10].

Lema 7.19. $\mathfrak{t}$ é um cardinal regular e $\mathfrak{p} \leq \mathfrak{t}$.

Em 2012, S. Shelah e M. Malliaris provaram em [MS16] que $\mathfrak{p}=\mathfrak{t}$, assim, a afirmação que provaremos não é mais forte do que a original. Apesar disso, daremos uma demonstração direta sem utilizar diretamente o fato de que $\mathfrak{p}=\mathfrak{t}$, cuja única demonstração conhecida é a deste artigo mencionado, que envolve técnicas avançadas de Teoria dos Modelos.

Teorema 7.20. Suponha que $X$ é $T_{2}$. Seja $D \subseteq X$ denso em $X$. Se $D^{\mathfrak{c}}$ é relativamente enumeravalmente compacto em $X^{\mathfrak{c}}$, então $[D]^{<\omega} \backslash\{\emptyset\}$ é relativamente enumeravelmente compacto em $\mathrm{CL}(X)$ e $\mathrm{CL}(X)$ é pseudocompacto.

Demonstração. Seja $f: \omega \rightarrow[D]^{<\omega} \backslash\{\emptyset\}$ uma sequência de subconjuntos não vazios de $D$ e seja $F_{n}=f(n)$. Seja $\mathcal{G}=\left\{g \in D^{\omega}: g(n) \in F_{n}\right\}$. Segue que $|\mathcal{G}| \leq \mathfrak{c}$. Como $D^{\mathfrak{c}}$ é relativamente enumeravelmente compacto em $X^{\mathfrak{c}}$, existe um ultrafiltro livre $p$ tal que para todo $g \in \mathcal{G}$, existe $p-\lim g$ em $X$. Seja $Z=\{p-\lim g: g \in \mathcal{G}\}$ e $F=\operatorname{cl} Z$. Afirmamos que $p-\lim f=F$. Então seja $U$ uma vizinhança aberta de $F$. Devemos verificar que $\left\{n \in \omega: F_{n} \in U\right\} \in p$. É suficiente provar esta afirmação para $U$ sub-básico. Então seja $W \subseteq \omega$ um aberto. Se $U=\{K \in \operatorname{CL}(X): K \subseteq U\}$, suponha por absurdo que $\left\{n \in \omega: F_{n} \in U\right\} \notin p$. Então $A=\left\{n \in \omega: F_{n} \backslash W \neq \emptyset\right\} \in p$. Seja $g \in \mathcal{G}$ tal que $g(n) \in F_{n} \backslash W$ para todo $n \in A$. Então $p-\lim g \in X \backslash W$, absurdo já que $Z \subseteq W$.

Agora suponha que $U=\{K \in \mathrm{CL}(X): K \cap W \neq \emptyset\}$. Como $F \cap W \neq \emptyset$, existem $x \in Z \cap W$ e $g \in \mathcal{G}$ tal que $x$ é $p$-limite de $g$. Segue que $p \ni\{n \in \omega: g(n) \in W\} \subseteq\left\{n \in \omega: F_{n} \cap W \neq \emptyset\right\}$. Isso prova que $[D]^{<\omega}\{\emptyset\}$ é relativamente enumeravelmente compacto. Para ver que $\mathrm{CL}(X)$ é pseudocompacto, note que para todo espaço $Y$, se existe um denso relativamente enumeravelmente compacto em $Y$, então $Y$ é pseudocompacto.

Lema 7.21. Para toda MAD family $\mathcal{A}, \omega^{\mathfrak{t}}$ é relativamente enumeravelmente compacto em $\Psi(\mathcal{A})^{\mathfrak{t}}$.

Demonstração. Seja $F \in(\omega)^{\mathfrak{p}}$. Mostraremos que existe um ultrafiltro livre $p$ tal que $p-\lim F$ existe en $\Psi(\mathcal{A})^{\mathfrak{t}}$. Para cada $\delta<\mathfrak{c}$, seja $g_{\alpha}=\pi_{\alpha} \circ F$, onde $\pi_{\alpha}$ é a $\delta$-ésima projeção.

Recursivamente, defina uma $\supset^{*}$-torre $\left(A_{\alpha}: \alpha<\mathfrak{p}\right)$ tal que para cada $\alpha<\mathfrak{c}, g_{\alpha} \mid A_{\alpha+1}$ converge como se segue: seja $A_{0}=\omega$. Suponha que definimos $A_{\alpha}$. Existe $A \in \mathcal{A}$ tal que $\left|A \cap g_{\alpha}\left[A_{\alpha}\right]\right|=\omega$. Seja $A_{\alpha+1}=g_{\alpha}^{-1}\left[A \cap g_{\alpha}\left[A_{\alpha}\right]\right]$. Para um passo limite $\alpha$, seja $A_{\alpha}$ uma pseudointerseção de $\left(A_{\beta}: \beta<\alpha\right)$. Seja $p$ um ultrafiltro livre contendo cada elemento da torre. Note que para cada $g \in \mathcal{G}, p-\lim g$ existe, portanto, $p-\lim F$ existe.

Combinando o teorema e o lema anterior, segue o teorema desejado como corolário.

Corolário 7.22. Sob $\mathfrak{t}=\mathfrak{c}, \mathrm{CL}(\Psi(\mathcal{A}))$ é pseudocompacto para toda MAD family $\mathcal{A}$.

Demonstração. Basta aplicar o teorema em $X=\Psi(\mathcal{A}), D=\omega$, notando que $\omega$ é denso em $\Psi(\mathcal{A})$. Pelo lema, $\omega^{\mathfrak{c}}=\omega^{\mathfrak{t}}$ é enumeravelmente relativamente compacto em $X^{\mathfrak{c}}$, então segue a tese.

\subsection{O Base Tree Lemma}

Nosso objetivo agora será provar que é consistente que existe uma MAD family $\mathcal{A}$ tal que $\mathrm{CL}(\Psi(\mathcal{A}))$ não é pseudocompacto. Nessa seção, introduziremos o pequeno cardinal h e provaremos o Base Tree Lemma. Adaptaremos as demonstrações de [Bla10]. 
Definição 7.5. Dizemos que $D \subseteq[\omega]^{\omega}$ é aberto se para todos $A, B \in[\omega]^{\omega}$, se $A \subseteq^{*} B$ e $B \in D$, então $A \in D$. Além disso, dizemos que $D$ é um denso se para todo $B \in[\omega]^{\omega}$ existe $A \in D$ tal que $A \subseteq \subseteq^{*} B$.

Note que $D$ é aberto denso se, e somente se:

1. $A, B \in[\omega]^{\omega}$, se $A \subseteq^{*} B$ e $B \in D$, então $A \in D$, e

2. para todo $B \in[\omega]^{\omega}$ existe $A \in D$ tal que $A \subseteq B$.

As palavras utilizadas na definição acima não foram escolhidas de forma aleatória. De fato, há uma topologia em $[\omega]^{\omega}$ que dá sentido a elas, como veremos abaixo.

Lema 7.23. Na notação acima, os subconjuntos abertos formam uma topologia em $[\omega]^{\omega}$ e os abertos definidos acima são, de fato, os abertos dessa topologia. Assim como os densos.

Demonstração. $\emptyset$ é aberto por vacuidade. $X=[\omega]^{\omega}$ é aberto pois se $A, B \in[\omega]^{\omega}, B \in X$ (o que sempre ocorre) e $A \subseteq^{*} B$, então $A \in X=[\omega]^{\omega}$. Se $A, B$ são abertos, então $A \cap B$ também é, pois se $C, D \in[\omega]^{\omega}$ são tais que $C \subseteq^{*} D \in A \cap B$, então $C \subseteq^{*} D \in A$ e $C \subseteq^{*} D \in B, \operatorname{logo}, C \in A, C \in B$, e, portanto, $C \in A \cap B$. Finalmente, se $\mathcal{A}$ é uma coleção de abertos, $B, C \in X$ e $B \subseteq{ }^{*} C \in \bigcup \mathcal{A}$, então existe $D \in \mathcal{A}$ tal que $B \subseteq \subseteq^{*} C \in D$, assim, $B \in D$, e, portanto, $B \in \bigcup \mathcal{A}$. Isso mostra que os abertos formam uma topologia.

Agora suponha que $D$ seja tal que para todo $B \in X$ existe $A \in D$ tal que $A \subseteq^{*} B$. Veremos que $D$ é denso. Seja $U$ um aberto não vazio. Seja $B \in U$ qualquer. Existe $A \in D$ tal que $A \subseteq^{*} B$, mas, como $U$ é aberto, isso implica que $A \in U$. Logo, $A \in U \cap D$.

Reciprocamente, suponha que $D$ é um denso. Veremos que para todo $B \in X$ existe $A \in D$ tal que $A \subseteq^{*} B$. Fixe $B$. Seja $U=\left\{A \in[\omega]^{\omega}: A \subseteq^{*} B\right\}$. É claro que $U$ é aberto não vazio. Logo, se $A \in D \cap U$, segue que $A \in D$ e $A \subseteq^{*} B$.

Agora, iremos introduzir o pequeno cardinal $\mathfrak{h}$.

Definição 7.6. $\mathfrak{h}$ é o menor cardinal para o qual uma interseção de $\mathfrak{h}$ abertos densos é vazia. $\mathfrak{h}$ é chamado de número de distributividade.

Quando trabalharmos com abertos densos, os lemas abaixo se tornam úteis e relacionam o pequeno cardinal com MAD families.

Lema 7.24. Seja $\mathcal{A}$ uma MAD family. Então $\mathcal{A} \downarrow=\left\{X \in[\omega]^{\omega}: \exists A \in \mathcal{A}\left(X \subseteq \subseteq^{*} A\right)\right\}$ é um aberto denso. Além disso, todo aberto denso contém um outro dessa forma.

Demonstração. É imediato que $\mathcal{A} \downarrow$ é um aberto. Vejamos que $\mathcal{A} \downarrow$ é denso. Seja $B \subseteq \omega$ infinito. Existe $A \in \mathcal{A}$ tal que $A \cap B$ é infinito. Temos que $A \cap B \in \mathcal{A} \downarrow$ então segue a tese. Para a segunda afirmação, seja $D$ um aberto denso. Pelo lema de Zorn, existe $\mathcal{A} \subseteq \mathscr{P}(D)$ almost disjoint family maximal em $D$. É claro que $\mathcal{A} \downarrow \subseteq D$ (pois $D$ é aberto). Precisamos apenas verificar que $\mathcal{A}$ é MAD. Suponha que não seja e tome $X \in[\omega]^{\omega}$ tal que para todo $A \in \mathcal{A}, A \cap X$ é finito. Como $D$ é aberto denso, existe $Y \in D$ tal que $Y \subseteq X$. Temos que $\mathcal{A} \cup\{Y\}$ viola a maximalidade de $\mathcal{A}$, o que é um absurdo.

Lema 7.25. Seja $\mathcal{A}$ uma MAD family e $A \in[\omega]^{\omega}$. Então $A \notin \mathcal{A} \downarrow$ se, e somente se, existem $B, C \in \mathcal{A}$ distintos tais que $|A \cap B|=|A \cap C|=\omega$.

Demonstração. Suponha que $A \notin \mathcal{A} \downarrow$. Como $\mathcal{A}$ é MAD, existe $B \in \mathcal{A}$ tal que $|B \cap A|=\omega$. Como $B \notin \mathcal{A} \downarrow$, temos que $|A \backslash B|=\omega$. logo, existe $C \in \mathcal{A}$ tal que $|C \cap(A \backslash B)|=\omega$. Isso implica que $B \neq C$ e que $|A \cap C|=\omega$.

Reciprocamente, suponha que $A \in \mathcal{A} \downarrow$. Existe $B \in \mathcal{A}$ tal que $A \subseteq^{*} B$. Se $C \in \mathcal{A}$ é tal que $A \subseteq{ }^{*} C$, temos que $A \subseteq{ }^{*} B \cap C$, logo, $|B \cap C|=\omega$. Assim, $B=C$ e segue a tese. 
Como corolário dos lemas acima, obtemos outra caracterização do cardinal $\mathfrak{h}$.

Corolário 7.26. $\mathfrak{h}=\min \{\kappa:$ Existe uma coleção $\mathscr{A}$ de $\mathrm{MAD}$ families tal que $|\mathscr{A}|=\kappa \mathrm{e}$ $\left.\forall X \in[\omega]^{\omega} \exists \mathcal{A} \in \mathscr{A}(\exists A, B \in \mathcal{A}(A \neq B \wedge|A \cap X|=|B \cap X|=\omega))\right\} \leq \mathfrak{c}$.

Demonstração. Mostremos primeiro a segunda desigualdade. Dado $X \subseteq \omega$ infinito, escolhamos arbitrariamente $X_{1}, X_{2}$ subconjuntos disjuntos de $X$ e seja $\mathcal{A}_{X}$ uma MAD family contendo $X_{1}, X_{2}$. Temos que $\mid\left\{A_{X}: X \in[\omega]^{\omega} \mid=\mathfrak{c}\right.$, logo, vale a desigualdade desejada.

Agora provaremos a igualdade. Começaremos provando $\leq$. Suponha que $\mathscr{A}$ é uma tal coleção de MAD families que define o lado direito e seja $\kappa=|\mathcal{A}|$. Afirmo que $\{\mathcal{A} \downarrow: \mathcal{A} \in \mathscr{A}\}$ possui interseção vazia. Com efeito, dado $X \in[\omega]^{\omega}$, existe $\mathcal{A} \in \mathscr{A}$ tal que existem $A, B$ distintos em $\mathcal{A}$ com $|X \cap A|=|X \cap B|=\omega$. Pelo lema anterior, segue que $X \notin \mathcal{A} \downarrow$. Isso prova $\leq$.

Para provar $\geq$, suponha que $\mathcal{D}$ é uma coleção de abertos densos de cardinalidade $\mathfrak{h}$ cuja interseção é vazia. Para cada $D \in \mathcal{D}$, seja $\mathcal{A}_{D}$ uma MAD family tal que $\mathcal{A}_{D} \downarrow \subseteq D$. Seja $\mathscr{A}=\left\{\mathcal{A}_{D}: D \in \mathcal{D}\right\}$. Temos que $|\mathcal{A}| \leq \mathfrak{h}$ e $\bigcap\left\{\mathcal{A}_{D} \downarrow: D \in \mathcal{D}\right\}$ é vazio. Assim, dado $X \in \mathcal{A}$, existe $D$ tal que $X \notin \mathcal{A}_{D} \downarrow$, e, pelo lema anterior, existem $A, B \in \mathcal{A}$ distintos tais que $|A \cap X|=|B \cap X|=\omega$. Assim, segue a tese.

É interessante encontrar cotas superiores e inferiores para $\mathfrak{h}$ para saber onde ele se situa em meio aos outros pequenos cardinais definidos no Capítulo 2.

Proposição 7.27. $\mathfrak{t} \leq \mathfrak{h}$.

Demonstração. Suponha que $\kappa<\mathfrak{t}$ e suponha que $\mathcal{D}$ é uma coleção de $\kappa$ abertos densos. $\bigcap \mathcal{D}$ é aberto, pois se $A \in \bigcap \mathcal{D}$ e $B \subseteq^{*} A$, como cada membro de $\mathcal{D}$ é aberto segue que $B \in \bigcap \mathcal{D}$. Escrevemos $\mathcal{D}=\left\{D_{\alpha}: \alpha \leq \kappa\right\}$. Recursivamente, tomamos $A_{\alpha} \in D_{\alpha}$ para $\alpha \leq \kappa$ de modo que $A_{0}$ seja arbitrário, definido $A_{\alpha}, A_{\alpha+1} \subseteq^{*} A_{\alpha}$ (o que é possível pela densidade), e se $\alpha$ for limite, tomemos $P$ pseudointerseção de $A_{\beta}(\beta<\alpha)$ e seja $A_{\alpha} \subseteq^{*} P \operatorname{com} A_{\alpha} \in D_{\alpha}$. Temos que $A_{\kappa} \in D_{\alpha}$ para todo $\alpha \leq \kappa$, pois se $\alpha \leq \kappa$, temos que $A_{\kappa} \subseteq^{*} A_{\alpha} \in \mathcal{D}_{\alpha}$, e este é aberto.

Assim, temos uma cota inferior para $\mathfrak{h}$. Ainda mostraremos uma cota superior menor (ou igual) à c. Mas antes veremos que h é regular. Para tanto, utilizaremos o seguinte lema, que por si só já tem a sua importância.

Proposição 7.28. A interseção de menos do que $\mathfrak{h}$ abertos densos é um aberto denso.

Demonstração. Seja $\mathcal{D}$ uma tal coleção de cardinalidade $\kappa<\mathfrak{h}$. $\bigcap \mathcal{D}$ é aberto, pois se $A \in \bigcap \mathcal{D}$ e $B \subseteq^{*} A$, como cada membro de $\mathcal{D}$ é aberto segue que $B \in \bigcap \mathcal{D}$. Para a densidade, fixe $X \in[\omega]^{\omega}$. Basta ver que $\bigcap_{\alpha<k}\left\{Y \in D_{\alpha}: Y \subseteq X\right\}$ é não vazio, pois então, tomando $Y$ na interseção, segue que $Y \in \mathcal{D}_{\alpha}$ para todo $\alpha<\kappa$ e $Y \subseteq X$. Então, verificaremos isso.

Seja $f: X \rightarrow \omega$ bijeção. Seja $g: \mathscr{P}(X) \rightarrow \mathscr{P}(\omega)$ dada por $g(A)=f[X]$. Afirmo que $\bigcap_{\alpha<k} g\left[D_{\alpha}\right]$ é não vazio. Para isso, basta ver que cada $g\left[D_{\alpha}\right]$ é um aberto denso. Suponha que $B \subseteq^{*} g(A)$ para algum $A \in D_{\alpha}$. Temos que $f^{-1}[B] \subseteq^{*} A \in D_{\alpha}$, logo, $f^{-1}[B] \in D_{\alpha}$ e, assim, $B \in g\left[D_{\alpha}\right]$. Assim, cada $g\left[D_{\alpha}\right]$ é aberto. Para ver que $g\left[D_{\alpha}\right]$ é denso, se $A \in \omega$, temos que $f^{-1}[A] \subseteq X$ e existe $B \in D_{\alpha}$ com $B \subseteq f^{-1}[A]$. Assim, $g(B) \subseteq A$ e $g(B) \in g\left[D_{\alpha}\right]$. Logo, $\bigcap_{\alpha<\kappa} g\left[D_{\alpha}\right]$ é não vazio. Tomando $Y$ na interseção, temos que $g^{-1}[Y] \in \bigcap_{\alpha<\kappa}\left\{A \in D_{\alpha}: A \subseteq X\right\}$ e segue a tese.

Corolário 7.29. h é regular.

Demonstração. Suponha por absurdo que não seja e seja $\kappa=\operatorname{cf} \mathfrak{h}<\mathfrak{h}$. Seja $f: \kappa \rightarrow \mathfrak{h}$ cofinal estritamente crescente e seja $\left\{D_{\alpha}: \alpha<\mathfrak{h}\right\}$ uma coleção de abertos densos cuja interseção é vazia. Pelo proposição anterior, definindo-se $C_{\alpha}=\bigcap_{\beta<\alpha} D_{\alpha}$, onde $C_{0}=[\omega]^{\omega}$, temos que $C_{\alpha}(\alpha<\kappa)$ é uma família decrescente de abertos densos cuja interseção é vazia. Assim, $C_{f(\alpha)}(\alpha<\kappa)$ também é, o que viola a minimalidade de $\mathfrak{h}$.

Para determinar uma cota superior para $\mathfrak{h}$ melhor do que $\mathfrak{c}$, provaremos que $\mathfrak{b} \geq \mathfrak{h}$. Na verdade, faremos um pouco mais do que isso. Introduziremos outro conhecido pequeno cardinal. 
Definição 7.7. $H \subseteq \omega$ é homogêneo para $f:[\omega]^{n} \rightarrow k$ se $f \mid[H]^{n}$ é constante. $H$ é quase homogêneo para $f$ se existe $F \subseteq \omega$ finito tal que $H \backslash F$ é homogêneo para $f$. par é o menor cardinal $\kappa$ para o qual existe $\mathcal{A} \subseteq 2^{[\omega]^{2}}$ de cardinalidade $\kappa$ tal que não existe $X \subseteq \omega$ infinito tal que $X$ é quase homogêneo para toda $f \in \mathcal{A}$.

Provaremos uma desigualdade entre $\mathfrak{h}$ e $\mathfrak{p a r}$, mas, para isso, precisaremos do famoso Teorema de Ramsey.

Teorema 7.30 (Teorema de Ramsey). Sejam $n, k$ naturais não nulos. Seja $f:[S]^{n} \rightarrow k$. Existe $H \subseteq S$ infinito tal que $f \mid[H]^{n}$ é constante.

Demonstração. Podemos supor que $S=\omega$.

Indução em $n$. Para $n=1$ a prova é imediata. Suponha que o teorema vale para $n$. Provaremos que ele vale para $n+1$. Para cada $a \in \omega$, seja $f_{a}: \omega \backslash\{a\}:[\omega]^{n} \Leftarrow \rightarrow k$ dada por $f_{a}(X)=f(X \cup\{a\})$. Por hipótese de indução, para todo $a \in \omega$ e para todo $S \subseteq \omega$ infinito, existe $H_{a}^{S} \subseteq \omega$ infinito tal que $f_{a} \mid\left[H_{a}^{S}\right]^{n}$ é constante.

Agora, recursivamente escolheremos subconjuntos infinitos de $\omega, S_{i}$, e números naturais $a_{i}$ como se segue: Seja $S_{0}=\omega$ e $a_{0}=0$. Definido $S_{i}, a_{i}$, seja $S_{i+1}=H_{i}^{S_{i}}$ e $a_{i+1}>a_{i}$ tal que $a_{i+1} \in S_{i+1}$. Está claro que $i \in \omega$ então $f_{a_{i}} \mid\left[\left\{a_{m}: m \geq i\right\}\right]^{n}$ é constante. Seja $G\left(a_{i}\right)$ o valor assumido por esta função neste conjunto. Como $G:\left\{a_{i}: i \in \omega\right\} \rightarrow k$, existe $S \subseteq\left\{a_{i}: i \in \omega\right\}$ infinito tal que $G$ é constante. Segue que $f \mid[S]^{n+1}$ é constante.

É comum pensar que $f$ é uma função que associa $n$-uplas não ordenadas de elementos de $S$ a um conjunto finito de $k$ cores. Neste contexto, $f$ costuma ser chamada de coloração, e se $H \subset S$ é tal que $f \mid[H]^{n}$ é constante, $H$ é dito ser monocromático.

Proposição 7.31. $\mathfrak{h} \leq \mathfrak{p a r} \leq \mathfrak{b}$.

Demonstração. Provaremos primeiro a segunda desigualdade. Seja $\mathcal{B} \subseteq \omega^{\omega}$ uma família ilimitada de tamanho b. Podemos assumir que se $g \in \mathcal{B}$ então $g$ é estritamente crescente. Para cada $g \in \mathcal{B}$, seja $\tilde{g}[\omega]^{2} \rightarrow 2$ dada por $\tilde{g}(\{x, y\})=0$ caso $x<y$ e $g(x)<y$, e $\tilde{g}(\{x, y\})=1$ caso $x<y$ e $y<g(x)$. Veremos que dado $H \subseteq \omega$ infinito, existe $g \in \mathcal{B}$ tal que $H$ não é quase homogêneo para $\tilde{g}$, o que completa a prova. Primeiramente, notemos que dada $g$, se $\tilde{g} \mid[J]^{2}$ é constante igual à 1 , então $J$ é finito, já que sendo $x=\min H$, temos que $y \leq g(x)$ para todo $y \in J \backslash\{x\}$. Assim, basta encontrar $g$ tal que para todo $F \subseteq \omega$ finito, $\tilde{g} \mid[H \backslash F]$ não é constante igual à 0 Suponha que isso não ocorre. Seja $h: \omega \rightarrow H$ tal que $|(x, h(x)) \cap H|=1$, e denotemos o elemento deste conjunto por $y_{x}$. Fixe $g \in \mathcal{B}$. Veremos que $g \leq^{*} h$, o que viola a escolha de $\mathcal{B}$. Fixe $n$ tal que se $x, y \geq n$ são distintos e $x, y \in H$ então $\tilde{g}(\{x, y\})=0$. Segue que se $x \geq n$ então $x<y_{h}<h(x)$. Mas também temos que $y_{h}, h(x) \in H$, logo, $g\left(y_{h}\right)<h(x)$, e, como $g$ é estritamente crescente, $g(x)<h(x)$, o que completa a prova.

Agora provaremos a primeira desigualdade. Suponha que $\kappa<\mathfrak{h}$. Veremos que $\kappa<$ par. Para cada $\alpha<\kappa$, fixe $f_{\alpha}:[\omega]^{2} \rightarrow 2$. Basta ver que existe $H$ infinito que é quase homogêneo para $f_{\alpha}$ para todo $\alpha<\kappa$. Para cada $\alpha<\kappa$, seja $D_{\alpha}=\left\{H \in[\omega]^{\omega}: H\right.$ é quase homogêneo para $\left.f_{\alpha}\right\}$. $D_{\alpha}$ é claramente aberto, e, pelo Teorema de Ramsey, é denso. Logo, existe $H \in \bigcap_{\alpha<\kappa} D_{\alpha}$ e segue a tese.

Tendo já provado diversas propriedades sobre o cardinal $\mathfrak{h}$, vamos iniciar os lemas preparatórios para provar o Base Tree Lemma. Para tanto, lembraremos das seguintes definições gerais sobre árvores e provaremos um último lema.

Definição 7.8. Seja $\leq$ uma ordem parcial em $T$. $T$ é uma árvore se dado $s \in T$ o conjunto $\{t \in T: t \leq s\}$ é bem ordenado por $\leq$. Nesse caso, ht $(t)$ é o tipo dessa boa ordem, $\operatorname{Lev}_{\alpha}(T)=\{t \in$ $T: \operatorname{ht}(t)=\alpha \operatorname{e~ht}(T)=\sup \{h t(t): t \in T\}$. Caso no nível 0 tenha apenas um elemento, dizemos que ele é a raiz de $T$.

Lema 7.32. Seja $\mathcal{A}$ uma MAD family. Existe uma MAD family $\mathcal{B}$ tal que: 
(a) Para todo $A \in \mathcal{A},|\{X \in B: X \subseteq A\}|=\mathfrak{c}$.

(b) Para todo $B \in \mathcal{B}$ existe $A \in \mathcal{A}$ com $B \subseteq A$.

(c) Para todo $X \subseteq \omega$, se $|\{A \in \mathcal{A}:|A \cap X|=\omega\}|=\mathfrak{c}$ então existe $B \in \mathcal{B}$ tal que $B \subseteq X$.

Demonstração. Primeiramente, se $|\mathcal{A}|<\mathfrak{c}$, seja para cada $A \in \mathcal{A}$ uma MAD family $\mathcal{B}_{A}$ em $A$ de cardinalidade $\mathfrak{c}$. Então $\mathcal{B}=\bigcup_{A \in \mathcal{A}} \mathcal{B}_{A}$ satisfaz o pedido.

Agora suponha que $|\mathcal{A}|=\mathfrak{c}$. Seja $\mathcal{X}=\{X \subseteq \omega:|\{A \in \mathcal{A}:|A \cap X|=\omega\}|=\mathfrak{c}\}$ e enumeremos $\mathcal{X}=\left\{X_{\alpha}: \alpha<\kappa\right\}$ de forma injetora. Define-se recursivamente $\phi\left(X_{\alpha}\right) \in\{A \in \mathcal{A}:|A \cap X|=$ $\omega\} \backslash\left\{\phi\left(X_{\xi}\right): \xi<\alpha\right\}$. Dessa forma, $\phi: \mathcal{X} \rightarrow \mathcal{A}$ é uma função injetora tal que $|\phi(X) \cap X|=\omega$ para todo $X \in \mathcal{X}$.

Para cada $X \in \mathcal{X}$, seja $\left\{\phi_{X}^{0}, \phi_{X}^{1}\right\}$ uma partição de $\phi(X)$ tal que ambos elementos são infinitos e tal que $\phi_{X}^{0} \subseteq X \cap \phi(X)$. Seja $\mathcal{B}_{\phi(X)}=\mathcal{B}_{X}^{\prime} \cup\left\{\phi_{X}^{1}\right\}$, onde $\phi_{X}^{1}$, onde $\mathcal{B}_{X}^{\prime}$ é uma MAD family em $\phi_{X}^{0}$ de cardinalidade $\boldsymbol{c}$. Note que $\mathcal{B}_{\phi(X)}$ é uma MAD family em $\phi(X)$. Se $A \notin \phi[\mathcal{X}]$, seja $\mathcal{B}_{A}$ uma MAD family em $A$ de cardinalidade $\mathfrak{c}$. Então $\mathcal{B}=\bigcup_{A \in \mathcal{A}} \mathcal{B}_{A}$ satisfaz o pedido. Para verificar a última condição, dado $X$ como no enunciado, temos que $X \in \mathcal{X}$. Basta tomar $B \in \mathcal{B}_{X}^{\prime}$.

Teorema 7.33 (Base Tree Lemma). Existe $T \subseteq[\omega]^{\omega}$ com as seguintes propriedades:

1. $T$, ordenada por $\supset^{*}$, é uma árvore de altura $\mathfrak{h}$ de raiz $\omega$.

2. Todo nível de $T$, exceto pela raiz, é uma MAD family de cardinalidade $\mathfrak{c}$.

3. Cada elemento de $T$ possui $\mathfrak{c} \supset$-sucessores imediatos.

4. Se $X \subseteq \omega$ é infinito, então existe $Y \in T$ tal que $Y \subseteq X$.

Uma árvore $T$ satisfazendo as condições acima de altura $\kappa$ é uma base tree de altura $\kappa$.

Demonstração. Sejam $D_{\alpha}^{\prime}(\alpha<\mathfrak{h})$ uma coleção de abertos densos com interseção vazia. Para cada $\alpha \in(0, \mathfrak{h})$, seja $D_{\alpha}=\bigcup_{\beta<\alpha} D_{\alpha}^{\prime}$. Temos que cada um destes é um aberto denso, que se $\alpha$ é limite, $D_{\alpha}=\bigcap_{0<\beta<\alpha} D_{\beta}$ e que $\bigcap_{0<\beta<\mathfrak{h}} D_{\beta}=\emptyset$.

Definimos recursivamente para $\alpha<\mathfrak{h}$ o conjunto $T_{\alpha}$, o que veremos mais tarde ser o nível $\alpha$ da árvore. Seja $T_{0}=\{\omega\}$. Se $0<\gamma<\mathfrak{h}$ e $T_{\beta}$ já foi definido para todo $\beta<\gamma$, seja $T_{\gamma}^{\prime}$ uma MAD family tal que $T_{\gamma}^{\prime} \downarrow \subseteq \bigcap_{\beta<\gamma} T_{\beta} \downarrow \cap D_{\gamma}$, e então seja $T_{\gamma}$ uma MAD family obtida aplicando o lema anterior à $T_{\gamma}^{\prime}$.

É fácil ver que se $\beta<\alpha<\mathfrak{h}$ então para todo $A \in T_{\alpha}$ existe $A^{\prime} \in T_{\alpha}^{\prime}$ e $B^{\prime} \in T_{\beta}$ tal que $A \subseteq A^{\prime} \subseteq{ }^{*} B^{\prime}$.

Provemos a seguinte afirmação: Para todos $\alpha, \beta<\mathfrak{h}$, se $\beta<\alpha, A \in T_{\alpha}, B \in T_{\beta}$, então $B \backslash A$ é infinito. Fixe $A, B$ como antes. Existe $A^{\prime} \in T_{\alpha}^{\prime}$ com $A \subseteq A^{\prime}$ e temos que $A^{\prime} \backslash A$ é infinito. Existe $B^{\prime} \in T_{\beta}$ com $A^{\prime} \subseteq B^{\prime}$. Se $B=B^{\prime}$ então $|B \backslash A|=\left|A^{\prime} \backslash A\right|=\omega$. Caso contrário, $\left|B \backslash B^{\prime}\right|=\left|B \backslash A^{\prime}\right|=$ $|B \backslash A|=\omega$, pois $T_{\beta}$ é uma MAD family.

Veremos agora que se $A \in T_{\alpha}$ para algum $\alpha<\mathfrak{h}$ então $\left\{B \in T: B \supset^{*} A \wedge B \neq A\right\}$ é uma boa ordem sob a relação $\supset^{*}$ de tipo $\alpha$, o que prova 1 . Pelo parágrafo anterior, temos que $\left\{B \in T: B \supset^{*} A \wedge B \neq A\right\} \subseteq \bigcup_{\beta \leq \alpha} T_{\beta}$. Como $T_{\alpha}$ é uma MAD family, vale que $\left\{B \in T: B \supset^{*}\right.$ $A \wedge B \neq A\} \subseteq \bigcup_{\beta<\alpha} T_{\beta}$. Também vimos que se $\beta<\alpha$ então $\left|\left\{B \in T: B \supset^{*} A \wedge B \neq A\right\} \cap T_{\beta}\right| \geq 1$. Veremos que vale a igualdade. Com efeito, se $B, B^{\prime}$ estão na interseção, como $A \subseteq^{*} B \cap B^{\prime}$ e $T_{\beta}$ é uma MAD family, segue que $B=B^{\prime}$. Para cada $\beta<\alpha$, seja $B_{\beta}$ o elemento desta interseção. Resta ver apenas que $\beta \rightarrow B_{\beta}$ é um isomorfismo de ordem. Se $\beta<\gamma<\alpha$, como vimos, existe $B^{\prime} \in T_{\beta}$ com $B_{\gamma} \subseteq^{*} B^{\prime}$. Então $B^{\prime} \cap B_{\beta}$ é infinito, o que implica que $B=B_{\beta}$. Pelo parágrafo acima, segue que $B_{\gamma} \nsubseteq^{*} B_{\beta}$, porém $B_{\beta} \subseteq^{*} B_{\gamma}$. Assim, segue a tese.

2. é claro. Para ver 3, fixe $\alpha<\mathfrak{h}$ e $A \in T_{\alpha}$. Primeiro, veremos que existe $B \in T_{\alpha+1}^{\prime} \operatorname{com} B \subseteq \subseteq^{*} A$. Primeiramente, note que $\bigcap_{\beta<\alpha+1} T_{\beta} \downarrow \cap D_{\alpha+1}=T_{\alpha} \downarrow \cap D_{\alpha+1}$. Existe $A^{\prime} \in D_{\alpha} \operatorname{com} A^{\prime} \subseteq^{*} A$ nessa interseção. Afirmo que existe $X \in T_{\alpha+1}^{\prime}$ tal que $X \subseteq^{*} A$. Tome $X \in T_{\alpha+1}^{\prime}$ tal que $X \cap A^{\prime}$ é infinito. 
Existe $B \in T_{\alpha}$ e $B^{\prime} \in D_{\alpha}$ com $X \subseteq^{*} B^{\prime} \subseteq^{*} B$. Logo, $B \cap A$ é infinito. Assim, segue que $X \subseteq^{*} B=A$. Agora, $X$ possui $\mathfrak{c}$ subconjuntos em $T_{\alpha+1}$, e segue que todos estes são sucessores imediatos de $A$.

Resta verificar 4. Fixe $X \subseteq \omega$ infinito. Construiremos uma subárvore $S$ de $T$ de altura $\omega$ como se segue: Define-se $S_{n}$ (que será o $n$-ésimo nível da sub-árvore) recursivamente de modo que:

i) $S_{0}=\{\omega\}$

ii) Se $Z \in S_{n}$, então $X \cap Z$ é infinito.

iii) $\left|S_{n}\right|=2^{n}$.

iv) Existe (um único) $\alpha_{n}<\mathfrak{h}$ tal que $S_{n} \subseteq T_{\alpha_{n}}$, e temos que se $n<m, \alpha_{n}<\alpha_{m}$.

v) Para todo $Z \in S_{n+1}$ existe $W \in S_{n} \operatorname{com} Z \subseteq^{*} W$.

vi) Para todo $Z \in S_{n}$ existem $Z^{\prime}, Z^{\prime \prime} \in S_{n+1}$ distintos com $Z \supset^{*} Z^{\prime}, Z^{\prime \prime}$.

Note que, feito isso, i), iv) e v) garantem que $S$ é uma árvore de raiz $\omega$, altura $\omega$ e de $n$-ésimo nível $S_{n}$, e iii), vi) garantem que cada ponto de $S$ possui exatamente dois sucessores imediatos. Para ver que podemos fazer essa construção recursiva, define-se $S_{0}$ como em i), e é claro que valem as i), ii), iii) e iv) para $n=0$, sendo $\alpha_{0}=0$.

Definido $T_{n}$, fixe $Z \in S_{n}$. Temos que $X \cap Z$ é infinito (pois ii)), logo, como $D_{\alpha}$ é uma sequência decrescente com interseção vazia, existe $\alpha_{Z}>\alpha_{n}$ tal que $Z \cap X \notin D_{\alpha_{Z}}$. Seja $\beta=\max \left\{\alpha_{Z}: Z \in S_{n}\right\}$, que, por iii), está bem definido e vale que $\alpha_{n}<\beta<\mathfrak{h}$. Como a sequência dos $D_{\alpha}$ é decrescente, dado $Z \in S_{n}$, temos que $Z \cap X \notin D_{\beta}$, portanto, $Z \cap X \notin T_{\beta} \downarrow \subseteq D_{\beta}$, o que implica que existem $Z^{\prime}, Z^{\prime \prime}$ distintos em $T_{\beta}$ quase-subconjuntos de $Z$. Seja $\alpha_{n+1}=\beta$ e $S_{n+1}=\left\{Z^{\prime}, Z^{\prime \prime}: Z \in S_{n}\right\}$, o que conclui a construção.

Podemos indexar $S$ (recursivamente) utilizando $2^{<\omega}$, escrevendo $S=\left\{Z_{s}: s \in 2^{\omega}\right\}$ de modo que $Z_{s} \in S_{|s|}$ e de modo que se $s \subseteq t$ são elementos de $2^{\omega}$ então $Z_{s} \supset^{*} Z_{t}$. Seja $\gamma=\sup \left\{\alpha_{n}: n \in \omega\right\}<\mathfrak{h}$, pois $\mathfrak{h}$ é regular. Dado $f \in 2^{\omega}$, seja $P_{f} \subseteq X$ uma pseudointerseção de $\left\{Z_{f \mid n} \cap X: n \in \omega\right\}$.

Temos que $P_{f} \subseteq^{*} Z_{f \mid n} \in T_{\alpha_{n}}$ para todo $n \in \omega$, logo, $P_{f} \in T_{\alpha_{n} \downarrow}$ para todo $n$, o que implica que $P_{f} \in \bigcap_{\beta<\gamma} T_{\beta} \downarrow \cap D_{\gamma}=\bigcap_{\beta<\gamma} T_{\beta} \downarrow$. Por construção, $T_{\gamma}^{\prime}$ é uma MAD family e $T_{\gamma}^{\prime} \downarrow \subseteq \bigcap_{\beta<\gamma} T_{\beta} \downarrow$. Fixe $Z_{f} \in T_{\gamma}^{\prime}$ tal que $Z_{f} \cap P_{f}$ é infinito. Como $Z_{f} \in T_{\gamma}^{\prime}$ temos que dado $n$ existe $U_{n} \in T_{\alpha_{n}} \operatorname{com} Z_{f} \subseteq^{*} U_{n}$. Como $Z_{f} \cap P_{f} \subseteq^{*} P_{f \mid n}$, segue que $U_{n}=P_{f \mid n}$. Assim, $Z_{f}$ é uma pseudointerseção de $\left\{Z_{f \mid n}: n \in \omega\right\}$ em $T_{\gamma}^{\prime}$. A coleção $\left\{Z_{f}: f \in \omega\right\} \subseteq T_{\gamma}^{\prime}$ é uma coleção de $\mathfrak{c}$ elementos distintos que intersectam $X$ infinitamente (pois cada um intersecta $P_{f} \subseteq X$ infinitamente). Logo, por construção, existe $Y \in T_{\gamma}$ tal que $Y \subseteq X$. Assim, segue a tese.

\subsection{Pseudocompacidade de $\operatorname{CL}(\Psi(\mathcal{A}))$ sob $\mathfrak{h}<\mathfrak{c}$}

Na seção 7.3 , vimos que é consistente que $\mathrm{CL}(\Psi(\mathcal{A}))$ é pseudocompacto para toda MAD family $\mathcal{A}$. Nesta seção, veremos que é consistente que existe uma MAD family $\mathcal{A}$ tal que $\operatorname{CL}(\Psi(\mathcal{A})$ ) não é pseudocompacto. Para isso, utilizaremos a hipótese de que $\mathfrak{h}<\mathfrak{c}$. No Apêndice B., vemos que é consistente com os axiomas de ZFC que $\mathfrak{h}<\mathfrak{c}$. Assim, estará provada a independência da existência de uma MAD family cujo hiperespaço de seu $\Psi$-espaço não é pseudocompacto.

Surpreendentemente, não se sabe se existe, em ZFC, uma MAD family $\mathcal{A}$ tal que $\operatorname{CL}(\Psi(\mathcal{A}))$ é pseudocompacto [Hru14].

Teorema $7.34(\mathfrak{h}<\mathfrak{c})$. Existe uma MAD family $\mathcal{A}$ tal que $\mathrm{CL}(\Psi(\mathcal{A}))$ não é pseudocompacto.

Demonstração. Seja $\mathcal{T}$ uma base tree de altura $\mathfrak{h}$. Para $A \subseteq 2^{<\omega}$, seja $\pi_{A}=\left\{n \in \omega: A \cap 2^{n} \neq \emptyset\right\}$. Seja $\mathcal{A} \subseteq\left[2^{<\omega}\right]^{\omega}$ ser tal que:

(1) $\mathcal{A}$ é uma MAD family (de subconjuntos de $2^{<\omega}$ ). 
(2) Todo $A \in \mathcal{A}$ é uma cadeia ou uma anticadeia em $2^{<\omega}$.

(3) $\pi_{A} \in \mathcal{T}$ para todo $A \in \mathcal{A}$

(4) Se $A, B \in \mathcal{A}$ e $A \neq B$ então $\pi_{A} \neq \pi_{B}$.

Para ver que $\mathcal{A}$ existe, construiremos por recursão. Enumeremos $[\omega]^{\omega}=\left\{X_{\alpha}: \alpha<\mathfrak{c}\right\}$. Para $\alpha<\mathfrak{c}$, definimos $g(\alpha) \in\{0,1\}$ e $A_{\alpha}$ como se segue: supondo que a construção está feita para todo $\beta<\alpha<\mathfrak{c}$, se $X_{\alpha} \cap A_{\beta}$ é finito para todo $\beta<\alpha$, seja $A_{\alpha} \subseteq X_{\alpha}$ cadeia ou anticadeia infinita tal que $\pi_{A_{\alpha}} \in \mathcal{T}$ e tal que para todo $\beta<\alpha, \pi_{A_{\beta}} \neq \pi_{A_{\alpha}}$. Isso é possível de ser feito pois como $X_{\alpha}$ é infinito, este possui uma cadeia infinita ou uma anticadeia infinita. Tomamos um subconjunto desta cadeia ou anticadeia de modo que a projeção esteja na árvore, e então tomamos um dos $\mathfrak{c}$ sucessores imediatos que seja distinto de todos os já escolhidos. Nesse caso seja $g(\alpha)=1$. A função $g$ serve como uma função indicadora de que um conjunto relevante foi construído nesse estágio. Caso contrário, seja $g(\alpha)=0$ e $X_{\alpha}=\emptyset$. Seja $\mathcal{A}=\left\{A_{\alpha}: \alpha \in g^{-1}[\{1\}]\right.$. É claro que $\mathcal{A}$ é uma almost disjoint family que satisfaz (2), (3) e (4). Devemos ver que ela é MAD. Dado $X \subseteq \omega$ infinito, existe $\alpha$ tal que $X=X_{\alpha}$. Se $g(\alpha)=0$, existe $\beta<\alpha$ tal que $A_{\beta} \cap X$ é infinito. Temos que $g(\beta)=1$, caso contrário teríamos que $A_{\beta} \cap X=\emptyset$, o que é absurdo. Caso contrário, $A_{\alpha}$ é um subconjunto infinito de $X_{\alpha}$ e segue a tese.

Seja $Y=\left\{2^{m}: m>0\right\} \subseteq \operatorname{CL}(\Psi(\mathcal{A}))$. Afirmo que todo ponto de $Y$ é isolado. Com efeito, dado $m,\left\{2^{m}\right\}=\left\langle 2^{m},\left\{s_{1}\right\}, \ldots,\left\{s_{2^{m}}\right\}\right\rangle$ onde $2^{m}=\left\{s_{1}, \ldots, s_{2^{m}}\right\}$. Temos que cada $2^{m}$ é finito, portanto fechado. Isso mostra que $Y$ é um aberto. Veremos que $Y$ é fechado, o que mostra que o espaço não é pseudocompacto. Para isso, basta ver que $Y$ não possui ponto de acumulação. Fixe $F \in \operatorname{CL}(\Psi(\mathcal{A}))$. Veremos que $F$ não é ponto de acumulação de $Y$. Primeiramente, notemos que se $s \in F \cap 2^{<\omega}$ então $U=\langle\Psi(\mathcal{A}),\{s\}\rangle$ é uma vizinhança de $f$ que intersecta no máximo um ponto de $Y$. Então resta apenas verificar o caso em que $F \subseteq \mathcal{A}$.

Caso 1: $|F|<\mathfrak{c}$. Nesse caso, existe $f \in 2^{\omega}$ tal que $A_{f}=\{f \mid n: n \in \omega\}$ intersecta finitamente todo elemento de $F$. Pois suponha que não. Então dado $f \in 2^{\omega}$ existe $B_{f} \in F \subseteq \mathcal{A}$ tal que $\left|A_{f} \cap B_{f}\right|=\omega$. Note que se $f \neq g$, então $B_{f} \neq B_{g}$, pois suponha que $B_{f}=B_{g}$. Como $A_{f} \cap B_{f}$ é infinito, $B_{f}$ possui infinitos elementos compatíveis, logo, por (2), é uma cadeia. Porém, como $B_{f}$ também contém infinitos elementos de $A_{g}$, temos que $B_{f}$ deve conter dois elementos incompatíveis, o que é absurdo. Assim, existe $f \in 2^{\omega}$ tal que $A_{f}=\{f \mid n: n \in \omega\}$ intersecta finitamente todo elemento de $F$. Seja:

$$
U=\left\{H \in \Psi(\mathcal{A}): H \cap \mathrm{cl}_{\Psi(\mathcal{A})}\left(A_{f}\right)\right\}=\left\langle\Psi(\mathcal{A}) \backslash \operatorname{cl}_{\Psi(\mathcal{A})}\left(A_{f}\right)\right\rangle
$$

Temos que $U$ é uma vizinhança de $F$ que não contém nenhum $2^{m}$ para $m>1$.

Caso 2: $|F|=\mathfrak{c}$.

Por (3) e (4), temos que $\left|\left\{\pi_{A}: A \in \mathcal{F}\right\}\right|=\mathfrak{c}>\mathfrak{h} \operatorname{logo}$ existem $A, B \in F$ tais que $\pi_{A}, \pi_{B}$ são elementos distintos em um mesmo nível de $\mathcal{F}$. Assim, existe $k>0$ tal que $\pi_{A} \cap \pi_{B} \subseteq k$. Seja $W=\left\langle\Psi(\mathcal{A}),\{A\} \cup A \backslash 2^{<k},\{B\} \cup B \backslash 2^{<k}\right\}$ é uma vizinhança de $F$ disjunta de $Y$. Assim, segue a tese.

Note que é possível enfraquecer a hipótese de $\mathfrak{h}<\mathfrak{c}$ para a existência de uma base tree sem cadeias de cardinalidade $\mathfrak{c}$.

\subsection{Um exemplo em ZFC}

Por enquanto, vimos resultados de consistência relativa sobre o problema inicial sobre se existe alguma relação entre a pseudocompacidade de $X^{\omega}$ e a de CL $(X)$. Nesta seção, veremos que, em ZFC, existe um subespaço $X$ de $\beta \omega$ tal que $X^{\omega}$ é pseudocompacto, porém $\operatorname{CL}(X)$ não é pseudocompacto. 
A construção não utilizará o conceito de MAD family, nem o de $\Psi$-espaço. Porém, o resultado foi incluído nesta dissertação pois acreditamos que ele é pertinente ao assunto em questão. Vimos que é consistente que para toda MAD family $\mathcal{A}, \mathrm{CL}(\Psi(\mathcal{A}))$ é pseudocompacto, e que é consistente que existe uma MAD family $\mathcal{A}$ tal que $\mathrm{CL}(\Psi(\mathcal{A}))$ não é pseudocompacto. Porém, não se sabe se, em ZFC, existe uma MAD family $\mathcal{A}$ tal que $\mathrm{CL}(\Psi(\mathcal{A}))$ é pseudocompacto, nem se é consistente que para toda MAD family $\mathcal{A}, \operatorname{CL}(\Psi(\mathcal{A}))$ não é pseudocompacto. Assim, mostraremos um exemplo de um subespaço $X$ de $\beta \omega$ cuja $\omega$-ésima potência é pseudocompacta, porém tal que $\mathrm{CL}(X)$ não é pseudocompacto. Tal exemplo é construído no artigo [HHR07]. Verificamos que é possível construir um espaço $X$ com essas propriedades tal que $X^{\kappa}$ é enumeravelmente compacto para todo $\kappa<\mathfrak{h}$ (em particular, $\kappa=\omega$ ). Tal construção aparecerá em um artigo que está em fase final de revisão para ser submetido para publicação.

Dado um ordinal $\alpha, \lim \alpha$ é o conjunto dos ordinais limites menores do que $\alpha$.

Teorema 7.35. Existe $X \subseteq \beta \omega$ tal que $X^{\omega}$ é pseudocompacto, porém $\operatorname{CL}(X)$ não é.

Demonstração. Enumeremos $\left(\omega^{\omega}\right)^{\omega}=\left\{f_{\alpha}: \alpha \in \lim \mathfrak{c}\right\}$, onde $f_{\alpha}=\left(f_{\alpha, n}: n \in \omega\right)$. Seja $F_{n}=$ $\left[2^{n}, 2^{n+1}\right)$ para cada $n \in \omega$, e seja $Y=\left\{F_{n}: n \in \omega\right\}$. Dado $U \subseteq \omega$, seja $\pi_{U}=\left\{n \in \omega: U \cap F_{n} \neq \emptyset\right\}$, e para um ultrafiltro $p$, seja $\pi(p)=\left\{\pi_{U}: U \in p\right\}$.

Afirmo que, para todo ultrafiltro $p, \pi(p)$ é um ultrafiltro: dado $U \in p$ e $V$ tal que $\pi_{U} \subseteq V$, temos que, adicionando em $U$, para cada $n \in V$, algum elemento de $F_{n}$, obtemos $U^{\prime}$ tal que $\pi_{U^{\prime}}=V$, e temos que $U^{\prime} \in p$. Notemos que $\pi_{U \cap V} \subseteq \pi_{U} \cap \pi_{V}$, logo, $\pi(p)$ é fechado por interseção. Temos que dado $U \in p, \pi(U) \neq \emptyset$, pois $\bigcup_{n \in \omega} F_{n}=\omega \backslash\{\emptyset\}$. Assim, $\pi(p)$ é filtro. Resta ver que $\pi(p)$ é ultrafiltro. Fixe $A \subseteq \omega$. Seja $U=\bigcup\left\{F_{n}: n \in A\right\}$, e seja $V=\bigcup\left\{F_{n}: n \in \omega \backslash A\right\} \cup\{0\}$. Temos que $U \cup V=\omega$, logo, um deles está em $p$. Porém, $\pi_{U}=A$ e $\pi_{V}=\omega \backslash A$, assim, $A \in \pi(p)$ ou $\omega \backslash A \in \pi(p)$.

Construiremos recursivamente para $\alpha \in \lim \mathfrak{c}$, um ultrafiltro livre $q_{\alpha}$ e um conjunto $X_{\alpha}=$ $\left\{p_{\alpha+m}: m \in \omega\right\} \subseteq \beta \omega$ tal que para cada $\alpha \in \lim \mathfrak{c}$ e para cada $m \in \omega$ :

(1) $p_{\alpha+m}=q_{\alpha}-\lim \left(f_{\alpha, n}(m): n \in \omega\right)$,

(2) Existe $U \in p_{\alpha+m}$ tal que $\left|F_{k} \cap U\right| \leq 1$ para todo $k \in \omega$,

(3) Para todo $\beta<\alpha$, existem $U \in p_{\alpha+m}$ e $V \in p_{\beta}$ tal que $\pi_{U} \cap \pi_{V}=\emptyset$.

Feito isso, seja $X=\omega \cup \bigcup\left\{X_{\alpha}: \alpha \in \lim \mathfrak{c}\right\}$. Veremos que $Y$ não tem ponto de acumulação em $\mathrm{CL}(X)$. Suponha que exista e seja ele $F$.

Temos que $F \cap \omega=\emptyset$, pois se $m \in F \cap \omega$, então $W=\langle X,\{m\}\rangle$ é uma vizinhança aberta de $F$. Se $m>0$, tome $k$ tal que $m \in F_{k}$. Então $W \cap Y=\left\{F_{k}\right\}$, o que é absurdo. Já se $m=0$, temos que $W \cap Y=\emptyset$, o que é absurdo. Então dividimos o problema em dois casos.

Caso 1: $F$ é enumerável.

Pela propriedade (2), para todo $p \in F$ existe $U_{p} \in p$ tal que $\left|F_{k} \cap U_{p}\right| \leq 1$ para todo $k \in \omega$. Afirmo que existe $K=\left\{x_{m}: m \in \omega\right\}$ tal que para cada $m, x_{m} \in F_{m}$ e tal que $\left|K \cap U_{p}\right|<\omega$ para todo $p \in F$.

Para ver isso, escrevamos $F=\left\{p_{n}: n \in \omega\right\}$ e $U_{p_{n}}=U_{n}$. Note que $\left|F_{n}\right|=2^{n+1}-2^{n}=2^{n}$. Para $n \geq 1$, tome $x_{n} \in F_{n} \backslash \bigcup_{k<n} U_{n}$, seja $x_{0}=0$ e segue a tese.

Seja $W=\{H \in \mathrm{CL}(X): H \cap \operatorname{cl} K=\emptyset\}=\langle X \backslash \operatorname{cl} K\rangle$. Note que como cada $p \in F$ é livre e como $K \cap U_{p}$ é finito, segue que $K \notin p$, e, portanto, $p \notin \mathrm{cl} K$. Logo, $F \in W$. Porém, $Y \cap W=\emptyset$ já que dado $m, F_{m} \cap \operatorname{cl} K \neq \emptyset$.

Caso 2: $F$ é não enumerável.

Por (3), existem $p, q \in F, U \in p$ e $V \in q$ tais que $\pi_{U} \cap \pi_{V}=\emptyset$. Seja $W=\langle X, \operatorname{cl} U \cap X, \operatorname{cl} V \cap X\rangle$. $w$ é uma vizinhança de $F$ disjunta de todo $F_{k}$.

Agora verificaremos que $X^{\omega}$ é pseudocompacto. Seja $\left(h_{n}: n \in \omega\right)$ uma sequência de elementos de $\omega^{\omega} \subseteq X^{\omega}$. Existe $\alpha \in \lim \mathfrak{c}$ tal que $f_{\alpha}=\left(h_{n}: n \in \omega\right)$. Tome $h \in X^{\omega}$ dada por 
$h(m)=q_{\alpha}-\lim f_{\alpha, n}(m)$, e (1) garante que $h \in X^{\omega}$ e é $q_{\alpha}$ limite de $\left(h_{n}: n \in \omega\right)$.

Resta apenas mostrar que podemos definir sequências de ultrafiltros como acima.

Suponha que $\alpha$ é um ordinal limite e que escolhemos $X_{\beta}, q_{\beta}$ para todo $\beta \in \lim \alpha$. Para todos $n, m \in \omega$, seja $g_{m}(n)=f_{\alpha, n}(m)$. Afirmo que existe $C \in[\omega]^{\omega}$ tal que:

(a) Para todo $m \in \omega$ existe $k \in \omega$ tal que $g_{m} \mid(C \backslash k)$ é constante ou injetora, e $\left|g[C \backslash k] \cap F_{j}\right| \leq 1$ para todo $j \in \omega$,

(b) Se $m \neq n$ são naturais, então $g_{m}\left|C={ }^{*} g_{n}\right| C$ ou $g_{m}[C] \cap g_{n}[C]={ }^{*} \emptyset$.

(c) Para todo $\beta<\alpha$ e $m \in \omega, \pi_{g_{m}[C]} \cap \pi_{V}=\emptyset$ para algum $V \in p_{\beta}$.

Para ver isso, seja $N=\left\{\pi(p) \in \omega^{*}:(\exists \beta<\alpha)\left(p \in X_{\beta}\right)\right\}$. Recursivamente, definiremos uma sequência decrescente $\left(A_{m}: m \in \omega\right)$ de subconjuntos infinitos de $\omega$ satisfazendo:

(i) $g_{m} \mid A_{m}$ é constante ou injetora, e existe $k \in \omega$ tal que $\left|g_{m}\left[A_{m}\right] \cap F_{k}\right| \leq 1$ para todo $k \in \omega$.

(ii) Se $k<m$, então $g_{m}\left|A_{m}={ }^{*} g_{k}\right| A_{m}$ ou $g_{m}\left[A_{m}\right] \cap g_{k}\left[A_{m}\right]={ }^{*} \emptyset$.

(iii) $\left(\pi_{g_{m}\left[A_{m}\right]}\right)^{*} \cap N=\emptyset$.

Seja $A_{-1}=\omega$. Assuma que $\left\{A_{k}: k<m\right\}$ foi construído para algum $m \in \omega$. Existe $D \subseteq A_{m-1}$ infinito tal que $g_{m} \mid D$ é constante ou injetora. Se $g_{m} \mid D$ é constante, é claro que $\left|g_{m}[D] \cap F_{k}\right| \leq 1$ para todo $k<m$ natural. Caso contrário, é possível tomar $E \subseteq D$ infinito tal que $\left|g_{m}[E] \cap F_{k}\right| \leq 1$. Em qualquer caso, mostra-se que existe $E \subseteq A_{m-1}$ infinito tal que $g_{m} \mid E$ é constante ou injetora e $\left|g_{m}[E] \cap F_{k}\right| \leq 1$ para todo $k<m$ natural. Agora, é possível tomar $B \subseteq E$ de modo a satisfazer (2) com $B$ no lugar de $A_{m}$.

Como $|N|<\mathfrak{c}$, temos que int $\operatorname{cl} N=\emptyset$. É possível, então, tomar $U \subseteq g_{m}[B]$ infinito tal que $U \subseteq g_{m}[B]$ e $\left(\pi_{U}\right)^{*} \cap N=\emptyset$. Sendo $A_{m}=g_{m}^{-1}[U]$, segue a tese.

Seja $C$ tal que $C \subseteq^{*} A_{m}$ para todo $m \in \omega$. Está claro que $C$ satisfaz (a) e (b). $C$ também satisfaz (c), pois dado $\beta<\alpha$ e $m \in \omega$, temos que $C \subseteq^{*} A_{m},\left(\pi_{g_{m}[C]}\right)^{*} \cap N=\emptyset$, o que implica que $\pi\left(p_{\beta}\right) \notin \pi_{g_{m}[C]}$, logo, existe $V \in p_{\beta}$ tal que $\pi_{V} \cap \pi_{g_{m}[C]}=\emptyset$.

Agora seja $q_{\alpha} \in C^{*}$ arbitrário e seja $p_{\alpha+m}=q_{\alpha}-\lim \left(g_{m}(n): n \in \omega\right)$ (o $q_{\alpha}$ limite existe pois o espaço $\beta \omega$ é compacto).

Assim, é claro que $q_{\alpha}$ e $X_{\alpha}$ satisfazem (1). Como $C \in q_{\alpha}$, segue que $g_{m}[C] \in p_{\alpha+m}$. Logo, por (a), (2) é satisfeito. Para verificar (3), temos que se $\beta<\alpha$, por (c), $\pi_{g_{m}[C]} \cap \pi_{V}=\emptyset$ para algum $V \in p_{\beta}$. Como $g_{m}[C] \in p_{\alpha+m}$, segue a tese. 


\section{Apêndice A}

\section{MA $\sigma$-Centrado}

Um princípio mais fraco do que o Axioma de Martin é o Axioma de Martin centrado, que restringe o axioma de Martin a uma classe menos geral do que a das pré-ordens com c.c.c. Para isso, vamos definir a noção de subconjunto centrado em uma pré-ordem.

Definição A.1. Seja $P$ uma ordem parcial. Dizemos que $S \subseteq P$ é centrado se, e somente se, para todos $s_{1}, \ldots, s_{n} \in A$ existe $p \in P$ tal que $p \leq s_{1}, \ldots, s_{n}$. Dizemos que $P$ é $\sigma$-centrado se $P=\bigcup_{n \in \omega} A_{n}$, onde cada $A_{n}$ é centrado.

Por exemplo, se tomarmos $P=[\omega]^{\omega}$ ordenado por $\subseteq^{*}$, então $S \subseteq P$ é centrados se, e somente se, possui a pif forte.

Lema A.1. Se $P$ é uma pré-ordem $\sigma$-centrado, então $P$ tem c.c.c.

Demonstração. Se $\mathcal{A}$ é uma anticadeia em $P$. Escrevamos $P=\bigcup_{n \in \omega} A_{n}$, onde cada $A_{n}$ é centrado. Seja $f: \mathcal{A} \rightarrow \omega$ tal que $f(a)$ é o menor natural $n$ pra o qual $a \in A_{n}$. Se $f(a)=f(b)$, temos que $a, b$ são compatíveis, logo, $a=b$. Como $f$ é injetora, segue que $|\mathcal{A}| \leq \omega$.

Assim, poderíamos definir o Axioma de Martin centrado, substituindo c.c.c. por $\sigma$-centrado. Tal definição não é necessária pois o cardinal $\mathfrak{p}$ já faz esse papel.

Proposição A.2. Seja $\mathfrak{m}_{\sigma}$ o menor cardinal $\kappa$ tal que $\mathrm{MA}_{P}(\kappa)$ é falso para alguma pré-ordem $\sigma$-centrada. Então $\mathfrak{p} \geq \mathfrak{m}_{\sigma}$.

Demonstração. A pré ordem utilizada para provar que $\mathfrak{m} \leq \mathfrak{p}$ é $\sigma$-centrada (Teorema 2.18), logo, obtivemos uma prova de que $\mathfrak{m}_{\sigma} \leq \mathfrak{c}$. Para ver isso, se $\kappa<\mathfrak{m}_{\sigma}$ e $\mathcal{A}$ é uma família com a propriedade da interseção finita forte de cardinalidade $\kappa$, seja $P=[\omega]^{<\omega} \times[\mathcal{A}]^{<\omega}$ ordenado por:

$$
\left(s^{\prime}, F^{\prime}\right) \leq(s, F) \leftrightarrow s \subseteq s^{\prime} \wedge F \subseteq F^{\prime} \wedge \forall x \in F\left(s^{\prime} \backslash s \subseteq x\right)
$$

Notemos novamente que se $(s, F)$ e $\left(s, F^{\prime}\right)$ forem elementos de $P$ com $s=s^{\prime}$, então $\left(s, F \cup F^{\prime}\right)$ é extensão comum. Logo, $A_{s}=\left\{(s, F): F \in[\mathcal{A}]^{<\omega}\right\}$ é centrado para todo $s \in[\omega]^{<\omega}$. Como $P=\bigcup_{s \in[\omega]<\omega} A_{s}$, segue que $P$ é $\sigma$-centrado e o resto da prova segue de forma análoga.

Para a outra direção, mais trabalhosa, precisaremos de algumas proposições adicionais.

Lema A.3. Suponha que $P$ é uma pré-ordem para a qual $\neg \mathrm{MA}_{P}(\kappa)$. Então existe $Q \subseteq P$ tal que $|Q| \leq \kappa$ e $\neg \mathrm{MA}_{Q}(\kappa)$. Além disso, se $P$ tem c.c.c., então $Q$ tem c.c.c., e se $P$ é $\sigma$-centrado, então $Q$ é $\sigma$-centrado.

Demonstração. Seja $\mathcal{D}$ uma coleção de $\kappa$ subconjuntos densos em $P$ tal que não existe um filtro $G$ em $P$ que intersecta cada elemento de $\mathcal{D}$.

Para cada $D \in \mathcal{D}$, seja $f_{D}: P \rightarrow D$ tal que $f(p) \leq p$ para todo $p \in P$. Além disso, seja $g: P \times P \rightarrow P$ tal que se $p, q$ são compatíveis, então $g(p, q) \leq p, q$. Seja $Q$ o fecho de qualquer 
subconjunto não vazio de $P$ pelas funções $f_{D}$ e $g$. Pela Proposição 1.9 , temos que $|Q| \leq \kappa$. Como $Q$ é fechado por $g$, temos que $p, q \in Q$ são compatíveis em $Q$ se, e somente se, são compatíveis em $P$, e, como $Q$ é fechado por $f_{D}$, temos que $\mathcal{D}^{\prime}=\{D \cap Q: D \in \mathcal{D}\}$ é uma coleção de $\leq \kappa$ densos. Se existisse um filtro $H$ em $Q$ intersectando cada elemento de $\mathcal{D}^{\prime}$, então $G=\{x \in P: \exists y \in H(y \leq x)\}$ seria um filtro em $P$ intersectando cada elemento de $\mathcal{D}$, absurdo.

Como toda anticadeia em $Q$ é uma anticadeia em $P$, temos que se $P$ tem c.c.c. então $Q$ tem c.c.c.

Se $P$ é $\sigma$-centrado, sejam $A_{n} \subseteq P(n \in \omega)$ centrados em $P$ tais que $P=\bigcup_{n \in \omega} A_{n}$. Temos que $Q=\bigcup_{n \in \omega}\left(A_{n} \cap Q\right)$ e cada $A_{n} \cap Q$ é $\sigma$-centrado, pois se $p, q \in A_{n} \cap Q$, temos que $p, q$ são compatíveis em $P, \log$, são em $Q$. Assim, $Q$ é $\sigma$-centrado.

Definiremos abaixo a noção de família ligada, que pode ser pensado como um oposto de anticadeia.

Definição A.2. Seja $P$ uma ordem parcial. $L \subseteq P$ é uma família ligada se para todos $p, q \in L$ existe $s \in P$ tal que $s \leq p, q$. Ou seja, $L$ é uma coleção de elementos dois-a-dois compatíveis.

Está claro que todo filtro é uma família ligada.

Lema A.4. Seja $P$ uma ordem parcial tal que para qualquer coleção $\mathcal{D}$ com $|\mathcal{D}| \leq \kappa$ de subconjuntos densos de $P$ fechados para baixo, existe uma família ligada $L$ intersectando cada $D \in \mathcal{D}$. Então vale $\mathrm{MA}_{P}(\kappa)$.

Demonstração. Seja $\mathcal{D}$ com $|\mathcal{D}| \leq \kappa$ de subconjuntos densos de $P$. Queremos ver que existe $G$ filtro em $P$ que intersecta cada denso. Enumeremos $\mathcal{D}=\left\{D_{\alpha}: \alpha<\kappa\right\}$. Para cada $\alpha$, seja $M_{\alpha} \subseteq D_{\alpha}$ uma anticadeia maximal nesse sentido (o que existe pelo lema de Zorn). Seja $C_{\alpha}=\{p \in P: \exists q \in$ $\left.M_{\alpha}(p \leq q)\right\}$. Afirmo que cada $C_{\alpha}$ é denso, pois suponha que não.

Tome $x$ tal que se $y \leq x$ então $y \notin C_{\alpha}$. Temos que não existe $y \in M_{\alpha}$ tal que $x \leq y$, do contrário teríamos que $x \in C_{\alpha}$. Tome $d \in D_{\alpha}$ tal que $d \leq x$. Temos que $d \notin M_{\alpha}$. Dado $m \in M \alpha$, temos que $d, m$ são incompatíveis, pois se fossem compatíveis existiria $n \in P$ com $n \leq m, d$. Como $n \leq m$, temos que $n \in C_{\alpha}$, e como $n \leq d \leq x$, temos que $n \notin C_{\alpha}$, o que é absurdo. Logo, $\{d\} \cup M_{\alpha}$ é uma anticadeia contida dem $D_{\alpha}$, o que viola a maximalidade de $M_{\alpha}$ visto que $d \notin M_{\alpha}$.

Assim, cada $C_{\alpha}$ é denso.

Observemos que uma interseção finita de densos fechados para baixo é um denso fechado para baixo. Para cada $s \in \kappa^{<\omega} \backslash\{\emptyset\}$, define-se recursivamente no domínio de $s$ densos $C_{s}$ e anticadeias $M_{s}$ como se segue: se $\operatorname{dom}(s)=1$, define-se $C_{s}=C_{s(0)}$ e $M_{s}=M_{s(0)}$. Definidos $C_{s}$ para todo $s \in \kappa^{<n} \backslash\{\emptyset\}$ com $n \leq 2$, dado $s \in \kappa^{n}$ define-se $C_{s}^{\prime}=\bigcap\left\{C_{t}: t \in \operatorname{ran}(s)^{<n} \backslash\{\emptyset\}\right\}$, que é denso, e então toma-se $M_{s}$ anticadeia maximal em $C_{s}^{\prime}$ e define-se $C_{s}=\left\{p \in P: \exists q \in M_{s}(p \leq q)\right\}$.

Notemos que dado $s, t \in k^{<\omega} \backslash\{0\}$, temos que $M_{s \frown t} \subseteq C_{s} \frown t \subseteq C_{s} \cap C_{t}$, e que $C_{s}=\{p \in P$ : $\left.\exists q \in M_{s}(p \leq q)\right\}$.

Seja $L$ família ligada tal que $L \cap C_{s} \neq \emptyset$ para todo $s \in k^{<\omega} \backslash\{0\}$. Para cada $s$, tome $q_{s} \in L \cap C_{s}$. Existe um único $m(s) \in C_{s}$ tal que $q_{s} \leq m(s)$.

Dados $s, t$, temos que $m(s \frown t) \leq m(s), m(t)$, pois, se por exemplo, $m(s \neg t) \not \leq m(s)$, como $m(s \frown t) \in C_{s}$ temos que existe $u \in M_{s}$ tal que $m(s \frown t) \leq u$ distinto de $m(s)$, logo, $m(s \frown t)$ e $m(s)$ são incompatíveis, o que é absurdo pois $q_{s \frown t} \leq m(s \frown t), q_{s} \leq m(s)$ e $q_{s}, q_{s \frown t}$ são compatíveis.

Seja $G=\left\{r \in P: \exists s \in \kappa^{<\omega} \backslash\{0\}\left(p_{\alpha} \leq r\right)\right\}$. Temos que $G$ é um filtro e para cada $\alpha$, $m((\alpha)) \in M_{\alpha} \cap G \subseteq D_{\alpha} \cap G$.

O lema abaixo é de fácil de demonstração e fica a cargo do leitor.

Lema A.5. Seja $P$ uma pré-ordem sem elemento máximo (ou seja, um elemento 1 tal que para todo $p \in P, p \leq 1$ e se $1 \leq p$ então $1=p$ ). Então tomando $1 \notin P$ e $Q=\{P\} \cup\{1\}$, estendendo a ordem $\leq$ de $P$ adicionando os pares $(1,1),(p, 1)$ para todo $p \in P$, obtém-se que $Q$ é uma pré ordem que é c.c.c. se, e somente se, $P$ for, e que é $\sigma$-centrada se, e somente se, $P$ for.

Lembremos da seguinte definição: 
Definição A.3. Seja $P$ uma pré-ordem. Dizemos que $p \in P$ é um átomo se, e somente se, para todos $r, s \leq p$, temos que $r, s$ são compatíveis.

Lema A.6. Suponha que $P$ é uma pré-ordem sem átomos. Então $P$ possui uma anticadeia infinita.

Demonstração. Fixe $p \in P$. Como $P$ não tem átomos, existem $r_{0}, p_{0} \leq p$ incompatíveis. Definidos $r_{n}, p_{n}$, sejam $r_{n+1}, p_{n+1} \leq p_{n}$ incompatíveis. Dessa forma, temos que $p_{0} \geq p_{1} \geq p_{2} \geq \ldots$ e dados $r_{i}, r_{j}$, temos que $r_{i}, r_{j}$ são incompatíveis, pois se $q \leq r_{i}, r_{j}$, sem perda de generalidade $i<j$, segue que $q \leq r_{i}, p_{i}$, o que é absurdo.

Lema A.7. Seja $P$ uma pré-ordem e suponha que $p$ possua um átomo $p$. Então existe um filtro $G$ que intersecta qualquer subconjunto denso de $P$.

Demonstração. Fixe $p$ como no enunciado e seja $G$ a coleção dos pontos compatíveis com $p$. $G$ é não vazio, pois $p \in G$. Se $u, v \in G$, existem $a, b \in P$ tais que $a \leq p, u, b \leq p, v$. Como $a, b \leq p$, então existe $c \in P$ tal que $c \leq a, b \leq p$, e como $c \leq p$, segue que $c \in G$. Assim, $c \leq u, v$ e $c \in G$. Além disso, se $u \in G$ e $u \leq v$, temos que existe $a \in P$ tal que $a \leq p, u$, e, portanto, existe $a \leq p, v$, assim, $v \in G$.

Se $D$ é denso, existe $d \in D$ tal que $d \leq p$, assim, $d, p$ são compatíveis, e, portanto, $d \in D \cap G$.

A demonstração do teorema abaixo é uma adaptação da demonstração encontrada em [Kun11], que por sua vez é uma adaptação da demonstração original do artigo [Bel81].

Teorema A.8. Seja $\mathfrak{m}_{\sigma}$ o menor cardinal $\kappa$ tal que $\mathrm{MA}_{P}(\kappa)$ é falso para alguma pré-ordem $\sigma$ centrada. Então $\mathfrak{p}=\mathfrak{m}_{\sigma}$.

Demonstração. Já vimos que $\mathfrak{p} \geq \mathfrak{m}_{\sigma}$. Resta ver que $\mathfrak{p} \leq \mathfrak{m}_{\sigma}$. Para isso, fixe $P$ pré-ordem $\sigma$-centrada. Pelos nossos lemas, podemos supor que $|P| \leq \kappa$, que $p$ possui elemento máximo, denotado por 1 , e que $P$ não tem átomos. Fixe $D_{\alpha}(\alpha<\kappa)$ uma família de subconjuntos densos fechados para baixo de $P$. Veremos que existe uma família ligada que intersecta cada um destes densos.

Podemos escrever $P=\bigcup_{l \in \omega} C_{l}$, onde cada $C_{l}$ é centrado. Podemos supor que para cada $l$, $1 \in C_{l}$. Para cada $p \in P$, seja $p_{\downarrow}=\{q \in P: q \leq p\}$. Para cada $\alpha<\kappa$ e $p \in P$, seja $B_{\alpha}(p)=\{l \in$ $\left.\omega: D_{\alpha} \cap C_{l} \cap p_{\downarrow} \neq \emptyset\right\}$.

Afirmo que dado $m \in \omega$, o conjunto $\mathcal{A}_{m}=\left\{B_{\alpha}(p): p \in C_{m} \wedge \alpha<\kappa\right\}$ possui a propriedade da interseção forte. Com efeito, fixemos $p_{0}, \ldots, p_{n} \in P, \alpha_{0}, \ldots, \alpha_{n}<\kappa$. Temos que ver que $\bigcap_{i \leq n} B_{\alpha_{i}}\left(p_{i}\right)$ é infinito. Seja $E=\bigcap_{i \leq n} D_{\alpha_{i}}$, que é um denso fechado para baixo. Como $C_{m}$ é centrado, seja $q \in P$ $\operatorname{com} q \leq p_{0}, \ldots, p_{n}$. Temos que $I:=\left\{l \in \omega: E \cap C_{l} \cap q_{\downarrow} \neq \emptyset\right\} \subseteq \bigcap_{i \leq n} B_{\alpha_{i}}\left(p_{i}\right)$. Como $P$ não tem átomos, tomemos uma anticadeia infinita $\left\{a_{j}: j \in \omega\right\} \subseteq q_{\downarrow}$. Para cada $j$, tomemos $b_{j} \leq a_{j}$ com $b_{j} \in E$. Para cada $j$, existe $l_{j} \operatorname{com} b_{j} \in C_{l_{j}}$. Como os $b_{j}$ são dois a dois incompatíveis, segue que se $j \neq k$ então $l_{j} \neq l_{k}$, e temos que $b_{j}$ atesta que $l_{j} \in I$.

Como $\left|C_{m}\right|, \kappa<\mathfrak{p}$, para cada $m$ seja $Z_{m}$ pseudointerseção de $\mathcal{A}_{m}$. Para cada $s \in \omega^{<\omega}$, seja $\lambda(s)$ o último elemento de $s$, sendo $\lambda(\emptyset)=0$. Seja $T=\left\{s \in \omega^{<\omega}: \forall n<\lambda(s)\left(s(n) \in Z_{\lambda(s \mid n)}\right)\right\}$. Notemos que $\emptyset \in T$ e que, por exemplo, $(3,1,4) \in T$ se, e somente se, $3 \in Z_{0}, 1 \in Z_{3}$ e $4 \in Z_{1}$.

Para cada $\alpha<\kappa$, recursivamente define-se $f_{\alpha}: T \rightarrow P$ tal que $f_{\alpha}(\emptyset)=1$ (o elemento máximo de $P$ ) e tal que, para definir-se $s \frown(l) \in T$ :

1. Se $l \in B_{\alpha}\left(f_{\alpha}(s)\right)$, então $f_{\alpha}(s \frown(l)) \in D_{\alpha} \cap C_{l} \cap f_{\alpha}(s)_{\downarrow}$.

2. Caso contrário, $f_{\alpha}(s \frown(l))=1$.

Note que, como $1 \in C_{l}$ para todo $l$, temos que para toda $s$ e para todo $\alpha, f_{\alpha}(s) \in C_{\lambda(s)}$.

Para cada $\alpha<\kappa$ e cada $s \in T$, se $s \frown(l) \in T$ então $l \in Z_{\lambda(s)} \subseteq^{*} B_{\alpha}\left(f_{\alpha}(s)\right)$ (pois $f_{\alpha}(s) \in C_{\lambda(s)}$ ). Então existe $\phi_{\alpha}(s) \in \omega$ tal que $l \in B_{\alpha}\left(f_{\alpha}(s)\right)$ para todo $l \geq \phi_{\alpha}(s)$ tal que $s \frown(l) \in T$.

Para cada $\alpha, \phi_{\alpha}: T \rightarrow \omega$ e T é enumerável, então, como $\mathfrak{p} \leq \mathfrak{b}$, tome $\Gamma: T \rightarrow \omega$ tal que $\phi_{\alpha} \leq^{*} \Gamma$ para todo $\alpha<\kappa$. Recursivamente em $n$, fixemos $g: \omega \rightarrow \omega$ tal que toda $g \mid n \in T$ e tal que $g(n)$ é o menor $l \geq \Gamma(g \mid n)$ tal que $(g \mid n) \frown(l) \in T$. 
Para cada $\alpha$, como $\phi_{\alpha} \leq^{*} \Gamma$, existe $k_{\alpha} \in \omega$ tal que $\phi_{\alpha}(g \mid n) \leq \Gamma(g \mid n)$ para todo $n \geq k_{\alpha}$. Para $n \geq k_{\alpha}$, temos que $g(n) \in B_{\alpha}\left(f_{\alpha}(g \mid n)\right)$ ) (pois $g \mid n \in T$, por escolha, $l=g(n) \in Z_{\lambda(g \mid n)}$ e $l \geq \Gamma(g \mid n) \geq \phi_{\alpha}(g \mid n)$, logo, pela definição de $\phi_{\alpha}$, como $g \mid(n+1) \in T$ segue que $l=g(n) \in B_{\alpha}\left(f_{\alpha}(s)\right)$, logo, por 1., temos que $f_{\alpha}(g \mid(n+1)) \leq f_{\alpha}(g \mid n)$.

Para cada $\alpha$, seja $p_{\alpha}=f_{\alpha}\left(g \mid\left(k_{\alpha}+1\right)\right.$. Então $p_{\alpha} \in D_{\alpha} \cap C_{g\left(k_{\alpha}\right)} \cap f_{\alpha}\left(g \mid k_{\alpha}\right)_{\downarrow}$, e seja $L=\left\{p_{\alpha}: \alpha<\kappa\right\}$. Temos que $L \cap D_{\alpha} \neq \emptyset$ para todo $\alpha$. Devemos ver que $L$ é uma família ligada. Sejam $\alpha, \beta$ e assuma que $k_{\alpha} \leq k_{\beta}$. Se $k_{\alpha}=k_{\beta}$, então $p_{\alpha}, p_{\beta} \in C_{g\left(k_{\alpha}\right)}$, logo, estes são compatíveis. Se $k_{\alpha}<k_{\beta}$, temos que como $f_{\alpha}(g \mid(n+1)) \leq f_{\alpha}(g \mid n)$ para todo $n \geq k_{\alpha}$, obtém-se $f_{\alpha}\left(g \mid\left(k_{\beta}+1\right)\right) \leq f_{\alpha}\left(g \mid\left(k_{\alpha}+1\right)\right)=p_{\alpha}$, e $\left.\left.f_{\alpha}\left(g \mid k_{\beta}+1\right)\right), f_{\beta}\left(g \mid k_{\beta}+1\right)\right)=p_{\beta}$ estão ambos na família centrada $C_{g\left(k_{\beta}\right)}$, e, portanto, são compatíveis. 


\section{Apêndice B}

\section{O Forcing de Cohen e pequenos cardinais}

Neste apêndice, definiremos e introduziremos os fatos básicos sobre modelos de Cohen. Veremos que, neste modelo, desde que $\mathrm{CH}$ valha no modelo base, $\mathfrak{c}=\kappa=\mathfrak{d}$ e $\omega_{1}=\mathfrak{b}$, o que, em particular, prova a consistência de $\omega_{1}=\mathfrak{a}=\mathfrak{b}<\mathfrak{d}=\mathfrak{c}$.

\section{B.1 O modelo de Cohen}

Definição B.1. Sejam $I, J$ quaisquer. Define-se $\operatorname{Fn}(I, J)=\bigcup_{F \in[I]<\omega} J^{F}$. Ou seja, $\operatorname{Fn}(I, J)$ é o conjunto das funções de um subconjunto finito de $I$ em $J$. Ordena-se $\operatorname{Fn}(I, J)$ pela inclusão reversa. Assim, $\emptyset$ é o maior elemento de $\operatorname{Fn}(I, J)$.

Notemos que, nesta ordem, dois elementos são compatíveis se, e somente se, eles são funções compatíveis e que $\mathrm{F}_{\mathrm{n}}(I, J)$ é absoluto para interpretações transitivas e enumeráveis de ZFC.

O (um) modelo de Cohen é uma extensão genérica de uma interpretação transitiva e enumerável de $M$ quando $I$ é infinito, $|J| \geq 2$ e $J$ é enumerável em $M$. Veremos que as extensões por Forcing por $\operatorname{Fn}(I, J)$ dessa forma só dependem da cardinalidade de $I$ e de $M$. Veremos ainda que a lei zero-um vale para forcing com $\operatorname{Fn}(I, J)$, ou seja, se $\phi$ é uma sentença, então $1 \Vdash \phi$ ou $1 \Vdash \neg \phi$. Então, qualquer extensão genérica por $\operatorname{Fn}(I, J)$ satisfaz exatamente as mesmas sentenças.

Lembremos da definição de delta sistema e do seu famoso lema associado.

Definição B.2. Dizemos que $D$ é um delta sistema de raiz $r$ se para todos $a, b \in D, a \cap b=r$.

Teorema B.1 (Lema do Delta Sistema). Seja $A$ uma coleção não enumerável de conjuntos finitos. Existe $D \subseteq A$ e $r$ tal que $D$ forma um delta sistema de raiz $r$ não enumerável.

Demonstração. Notemos que existe $n>0$ natural tal que $\{s \in A:|s|=n\}$ é não enumerável (caso contrário, $A$ seria enumerável). Assim, basta provarmos, por indução, o seguinte lema: Para todo $n>0$ natural, se $A$ é uma coleção não enumerável de conjuntos de $n$ elementos então existem $D \subseteq A$ de mesma cardinalidade de $A$ e $r$ tais que $D$ é um delta sistema de raiz $r$. Provaremos isso por indução em $n$.

Para $n=1$, temos que $A$ é um delta sistema de raiz $\emptyset$.

Suponha que o lema é verdadeiro para um natural $n$ e seja $A$ uma coleção não enumerável de conjuntos de cardinalidade $n+1$. Dividimos a prova em dois casos.

Caso 1: Existe $B \subseteq A$ não enumerável tal que existe $x$ tal que $\forall F \in B(x \in F)$. Nesse caso, seja $B^{\prime}=\{F \backslash\{x\}: F \in B\}$. Temos que $B^{\prime}$ é uma coleção não enumerável de conjuntos de cardinalidade $n$, logo, existe $D^{\prime} \subseteq B^{\prime}$ não enumerável que forma um delta sistema de raiz $r$. Temos que $\left\{F \cup\{x\}: F \in D^{\prime}\right\} \subseteq B \subseteq A$ é um delta sistema de raiz $r \cup\{x\}$.

Caso 2: Para todo $B \subseteq A$ não enumerável e para todo $x$, existe $F \in B$ tal que $x \notin F$.

Recursivamente, construiremos $F_{\alpha} \in A$ para $\alpha<\omega_{1}$ dois a dois disjuntos. Feito isso, obtivemos um delta sistema de raiz $\emptyset$. Para ver que é possível construir essa sequência, definimos-a como se segue: 
Suponha definidos $F_{\beta}$ para todo $\beta<\alpha$ para algum $\alpha<\omega_{1}$ de modo que se $\beta<\beta^{\prime}<\alpha$ então $F_{\beta} \cap F_{\beta^{\prime}}=\emptyset$. Afirmo que existe $F \in A$ tal que para todo $\beta<\alpha, F \cap F_{\alpha}=\emptyset$. Pois suponha que não. Seja $U=\bigcup_{\beta<\alpha} F_{\alpha}$. Temos que $U$ é enumerável. Por hipótese de absurdo, para todo $F \in A$ existe $x_{F} \in U \cap F$. Como $U$ é enumerável e $A$ não enumerável, existe $B \subseteq A$ não enumerável tal que para todos $F, G \in B, x_{F}=x_{G}$. Assim, $B$ é um subconjunto não enumerável de $A$ tal que todos os elementos possuem um elemento em comum, o que viola a hipótese. Logo, existe $F$ como queremos. Sendo $F=F_{\alpha}$, completa-se a recursão.

Lema B.2. $\operatorname{Fn}(I, J)$ tem a condição da cadeia enumerável para quaisquer $I, J$ tal que $J$ é enumerável. Logo, forcings com essas ordens preservam cofinalidades e cardinalidades.

Demonstração. Seja $A \subseteq \operatorname{Fn}(I, J)$ não enumerável. Considere $B=\{\operatorname{dom} s: s \in A\}$. Define-se $C, r$ como se segue:

Se $B$ é enumerável, existe $r \in[I]^{<\omega}$ tal que $C=\{s \in A: \operatorname{dom} s=r\}$ é não enumerável.

Se $B$ é não enumerável, pelo lema do delta sistema existe $C^{\prime} \subseteq B$ não enumerável e $r \in[I]^{<\omega}$ tal que para todos $u, v \in C^{\prime}, u \cap v=r$. Nesse segundo caso, seja $C=\left\{s \in A: \operatorname{dom} s \in C^{\prime}\right\}$.

Em qualquer um dos dois casos, existe $C \subseteq A$ não enumerável tal que para todos $s, t \in C$, temos que $\operatorname{dom} s \cap \operatorname{dom} t=r$. Temos que $\{s \mid r: s \in C\} \subseteq J^{r}$. Como $J^{r}$ é enumerável, existe $s_{0} \in J^{r}$ tal que $D=\left\{s \in C: s \mid r=s_{0}\right\}$ é não enumerável. Se $s, t \in D$, temos que $\operatorname{dom} s \cap \operatorname{dom} t=r \mathrm{e}$ $s|r=t| r=s_{0}$, portanto, $s, t$ são compatíveis. Assim, $A$ não é uma anticadeia.

Logo, toda anticadeia de $\operatorname{Fn}(I, J)$ é enumerável e segue a tese.

Lema B.3. Seja $M$ uma interpretação transitiva e enumerável de ZFC e $I, J \in M$, com $I$ infinito e $J$ não vazio. Seja $P=\operatorname{Fn}(I, J)$ e $G$ um filtro $P$-genérico sobre $M$. Então $f=\bigcup G$ é uma função sobrejetora de $I$ em $J$.

Demonstração. $G$ é uma coleção de funções compatíveis, logo, $f=\bigcup G$ é uma função de um subconjunto de $I$ em $J$. Para cada $i \in I$, seja $D_{i}=\{s \in P: i \in \operatorname{dom} s\}$. $D_{i}$ é denso, pois dado $s \in P$, se $i \notin \operatorname{dom} s$, então $s \cup\{(i, j)\}$ é uma extensão de $s$ para todo $j \in J$. Logo, existe $s \in G$ com $i \in \operatorname{dom} s$, e, portanto, $i \in \operatorname{dom} f$. Como $i$ é arbitrário, $\operatorname{dom} f=I$.

Dado $j \in J$, seja $E_{j}=\{s \in P: j \in \operatorname{ran} s\}$. Temos $E_{j}$ é denso pois dado $s \in P$, temos que $s \cup\{(i, j)\}$ é uma extensão de $s$ para todo $i \in I \backslash \operatorname{dom} s$, que é não vazio já que $I$ é infinito. Isso mostra que a imagem de $f$ é $J$.

O lema abaixo nos mostra que se $I$ é enumerável e $2 \leq|J| \leq \omega$, então as extensões genéricas dadas por $\operatorname{Fn}(I, J)$ independem da escolha de $I, J$.

Lema B.4. Seja $P=\omega^{<\omega}$ a subordem de $\operatorname{Fn}(\omega, \omega)$. Seja $Q$ um forcing poset enumerável sem átomos. Então existe $i: P \rightarrow Q$ imersão densa.

Demonstração. Denotemos por 1 o elemento máximo de $Q$. Enumeremos $Q=\left\{p_{n}: n \in \omega \backslash\{0\}\right\}$. Definiremos recursivamente funções $i_{n}$ para $n \in \omega$ de modo que:

a) $i_{n}: \omega \leq n \rightarrow Q$.

b) Se $n \geq 1$, existe $s \in \omega \leq n$ tal que $i_{n}(s) \leq p_{n}$.

c) Se $s, t \in \omega \leq n$ e $s \leq t$ (ou seja, $t \subseteq s$ ) então $i_{n}(s) \leq i_{n}(t)$.

d) Se $n \leq m$ então $i_{n} \subseteq i_{m}$.

e) Para todo $s \in \omega^{n}$, temos que $i_{n+1}\left[\left\{s^{\frown}(j): j \in \omega\right\}\right]$ é uma anticadeia infinita maximal de $i_{n}(s) \downarrow$, e $i_{n+1} \mid \omega^{n+1}$ é injetora.

f) $i_{0}(\emptyset)=1$. 
Feito isso, seja $i=\bigcup_{n \in \omega} i_{n}$. Temos que d) garante que $i$ é função, a) e b) garantem que $\operatorname{dom} i=\omega$ e que $i\left[\omega^{<\omega}\right] \subseteq Q$ é denso. Para ver que $i$ preserva incompatibilidade, sejam $s, t \in \omega^{<\omega}$ incompatíveis. Seja $n=\max \{m \in \omega: s|m=t| m\}$. O item e) nos garante que $i(s \mid(n+1))$ e $i(t \mid(n+1))$ são incompatíveis, e por d), temos que $i(s)$ e $i(t)$ são incompatíveis (pois se fossem compatíveis, as duas condições anteriores também seriam compatíveis).

Define-se $i_{0}$ como em $\mathrm{f}$ ). Suponha que $i_{m}$ foi definida para todo $m \leq n$. Devemos definir $i_{n+1}$. Define-se $i_{n+1} \mid \omega^{\leq n}=i_{n}$, o que garante d). Resta definir $i_{n+1} \mid \omega^{n+1}$. Notemos que se e) for satisfeito, então c) também será.

Chamemos de $(*)$ o seguinte parágrafo:

(*) Como $Q$ não tem átomos, para todo $s \in \omega^{n}$ existe uma anticadeia infinita maximal em $i(s) \downarrow$. Escolhendo uma anticadeia maximal para cada $s$, define-se $i_{n+1}\left(s^{\frown}(j)\right)$ para cada $j \in \omega$ de modo que $i_{n+1}[\{s \frown(j): j \in \omega\}]$ é uma anticadeia infinita maximal de $i_{n}(s) \downarrow$, e $i_{n+1} \mid \omega^{n+1}$ é injetora.

Terminar a construção aplicando (*) não é o suficiente pois temos que fazer b) ser satisfeito. Então dividimos a construção em casos:

Caso 1: Existe $s \in \omega^{\leq n}$ tal que $i_{n}(s) \leq p_{n+1}$. Terminando a construção utilizando (*), temos que e) e b) são satisfeitos.

Caso 2: Não existe $s \in \omega^{\leq n}$ tal que $i_{n}(s) \leq p_{n+1}$. Seja $k=\max \left\{\exists t \in \omega^{<\omega}\left(i_{n}(t) \geq p_{n+1}\right)\right\}$, e $t \in \omega^{k}$ tal que $i_{n}(t) \geq s$. Note que $k$ está bem definido pois $i(\emptyset)=1$. Dividimos este caso em dois subcasos:

Subcaso 1: $k=n$. Neste caso, basta terminarmos a prova com o parágrafo $(*)$ com a exigência adicional de que $p_{n+1} \downarrow \cap i_{n+1}[\{t \frown(j): j \in \omega\}] \neq \emptyset$.

Subcaso 2: $k<n$. Neste caso, definiremos recursivamente duas sequências finitas $t_{j}(k \leq j \leq n)$ satisfazendo:

i) Para todo $j$ tal que $k \leq j \leq n, q_{j} \in Q$ e $t_{j} \in \omega^{j}$.

ii) $t_{k}=t, q_{k}=p_{n+1}$.

iii) Para todo $j$ tal que $k<j \leq n, q_{j} \leq i_{n}\left(t_{j}\right), q_{j-1}$.

Antes de provar que isso é possível, note que, feito isso, $q_{n} \leq i\left(t_{n}\right)$, sendo $t_{n} \in \omega^{n}$, e $q_{n} \leq q_{n-1} \leq$ $\cdots \leq q_{k}=p_{n+1}$, assim, para completar a prova basta aplicar $(*)$ com a exigência adicional de que $q_{n} \downarrow \cap i_{n+1}\left[\left\{t_{n}(j): j \in \omega\right\}\right] \neq \emptyset$. Agora provaremos que é possível construir recursivamente estas sequências.

Define-se $q_{k}, t_{k}$ como em ii). Definidos $q_{j}, t_{j}$ com $k \leq j<n$, temos que $q_{j} \leq i_{n}\left(t_{j}\right)$. Temos que $q_{j} \notin i_{n+1}\left[\left\{t_{j}(l): l \in \omega\right\}\right]$, caso contrário, teríamos que existe $s \in \omega \leq n$ tal que $i_{n}(s) \leq p_{n+1}$, o que é absurdo. Assim, $q_{j} \notin i_{n+1}\left[\left\{t_{j}(l): l \in \omega\right\}\right]$ e este conjunto é uma anticadeia maximal, existe $l \in \omega$ e $q_{j+1} \in Q$ tal que $q_{j+1} \leq i\left(t_{j}(l)\right), q_{j}$. Sendo $t_{j+1}=t_{j}(l)$, segue a tese.

Vamos agora trabalhar para mostrar que para todos $I, J \operatorname{com}|I|=\kappa$ fixado e $2 \leq|J| \leq \omega$ qualquer, $\operatorname{Fn}(I, J)$ nos dá as mesmas extensões genéricas. Assim, as propriedades de forcing da ordem $\operatorname{Fn}(I, J)$ com $2 \leq|J| \leq \omega$ dependem apenas da cardinalidade de $I$.

Definição B.3. Seja $P$ um forcing poset e $\kappa$ um cardinal. Define-se $\operatorname{FP}(P, \kappa)=\left\{f \in P^{\kappa}: \mid\{\alpha<\kappa\right.$ : $f(\alpha) \neq 1\} \mid<\omega\}$ e, para $f \in \operatorname{FP}(P, \kappa)$, define-se $\operatorname{supp}(f)=\{\alpha<\kappa: f(\alpha) \neq 1\}$. Ordena-se $\operatorname{FP}(P, \kappa)$ coordenada-a-coordenada. Note que a $\kappa$-sequência constante igual à 1 é o elemento máximo de $\mathrm{FP}(P, \kappa)$.

Lema B.5. Sejam $P$ forcing posets e $\kappa$ um cardinal infinito. Para todos $f, g \in \operatorname{FP}(P, \kappa)$, temos que $f \perp g$ se, e somente se, existe $\alpha<\kappa$ tal que $f(\alpha) \perp g(\alpha)$.

Demonstração. Suponha que existe $\alpha<\kappa$ tal que $f(\alpha) \perp g(\alpha)$. Então não existe $h \in \operatorname{FP}(P, \kappa)$ tal que $h \leq f, g$, caso contrário, teríamos que $h(\alpha) \leq f(\alpha), g(\alpha)$, o que é absurdo. Assim, $f \perp g$. 
Reciprocamente, suponha que para todo $\alpha<\kappa$ existe $p_{\alpha} \in P$ com $p_{\alpha} \leq f(\alpha), g(\alpha)$. Temos que, definindo-se $h: \kappa \rightarrow P$ dada por $h(\alpha)=q_{\alpha}$ para $\alpha \in \operatorname{supp} f \cup \operatorname{supp} g, h(\alpha)=1$ caso contrário, segue que $h \in \operatorname{FP}(P, \kappa)$ e $h \leq f, g$.

Lema B.6. Sejam $P, Q$ forcing posets e $\kappa$ um cardinal infinito. Suponha que $i: P \rightarrow Q$ é uma imersão densa. Seja $j: \operatorname{FP}(P, \kappa) \rightarrow \operatorname{FP}(Q, \kappa)$ dada por $j(f)(\alpha)=i(f(\alpha))$ é uma imersão densa.

Demonstração. Sejam $1_{P}, 1_{Q}$ os elementos máximos, respectivamente, de $P, Q$. Primeiramente, notemos que se $f \in \mathrm{FP}(P, \kappa)$, então $j(f) \in \mathrm{FP}(Q, \kappa)$. Isso ocorre pois, como $i\left(1_{P}\right)=1_{Q},\{\alpha<\kappa$ : $\left.i(f(\alpha)) \neq 1_{Q}\right\} \subseteq\left\{\alpha<\kappa: f(\alpha) \neq 1_{P}\right\}$.

Temos que $j$ preserva a ordem: Sejam $f, g \in \operatorname{FP}(P, \kappa)$ tais que $f \leq g$. Dado $\alpha$, temos que $f(\alpha) \leq g(\alpha)$. Assim, $j(f)(\alpha)=i(f(\alpha)) \leq i(g(\alpha))=j(g)(\alpha)$. Como $\alpha$ é arbitrário, segue que $i(f) \leq i(g)$.

Temos que $j$ preserva a incompatibilidade: Suponha que $f, g \in \operatorname{FP}(P, \kappa)$ são tais que $f \perp g$. Existe $\alpha<\kappa$ tal que $f(\alpha) \perp g(\alpha)$. Assim, $j(f)(\alpha)=i(f(\alpha)) \perp i(g(\alpha))=j(g)(\alpha)$, e, portanto, $j(f) \perp j(g)$.

$j$ é densa: tome $h \in \operatorname{FP}(Q, \kappa)$. Para cada $\alpha \in \operatorname{supp} h$, seja $p_{\alpha} \in P$ tal que $i\left(p_{\alpha}\right) \leq h(\alpha)$. Seja $f: \kappa \rightarrow P$ dada por $f(\alpha)=p_{\alpha}$, caso $\alpha \in \operatorname{supp} Q$, e $f(\alpha)=1_{P}$, caso contrário. Temos que $f \in \operatorname{FP}(P, \kappa)$ e $i(f) \leq h$.

Lema B.7. Seja $\kappa$ um cardinal infinito. Então:

a) Para todos $I, I^{\prime}$ tais que $|I|=\left|I^{\prime}\right|$ e para todo $J$, temos que $\operatorname{Fn}(I, J)$ é isomorfo à $\operatorname{Fn}\left(I^{\prime}, J\right)$.

b) $\operatorname{FP}(\operatorname{Fn}(\omega, J), \kappa)$ é isomorfo à $\operatorname{Fn}(\omega \times \kappa, J)$.

Demonstração. a) Seja $u: I \rightarrow I^{\prime}$ bijeção. Segue que $\phi: \operatorname{Fn}\left(I^{\prime}, J\right) \rightarrow \operatorname{Fn}(I, J)$ dada por $\phi(s)=s \circ u$ é isomorfismo.

b) Seja $\phi: \operatorname{FP}(\operatorname{Fn}(\omega, J), \kappa) \rightarrow \operatorname{Fn}(\omega \times \kappa, J)$ tal que $\phi(f):\{(n, \alpha) \in \omega \times \operatorname{supp} f: n \in$ $\operatorname{dom} f(\alpha)\} \rightarrow J$ é dada por $\phi(f)(n, \alpha)=f(\alpha)(n)$. Está claro que $\phi$ está bem definida.

$\phi$ preserva a ordem: Suponha que $f, g \in \operatorname{FP}(\operatorname{Fn}(\omega, J), \kappa)$ são tais que $f \leq g$. Devemos ver que $\phi(g) \subseteq \phi(f)$. Tome $(n, \alpha) \in \operatorname{dom} \phi(g)$. Temos que $n \in \operatorname{dom} f(\alpha)$. Como $f(\alpha) \leq g(\alpha)$, segue que $g(\alpha) \subseteq f(\alpha)$. Assim, $n \in \operatorname{dom} f(\alpha)$ e $\alpha \in \operatorname{supp} f$. Assim, $(n, \alpha) \in \operatorname{supp} \phi(f)$ e $\phi(f)(n, \alpha)=$ $f(\alpha)(n)=g(\alpha)(n)=\phi(g)(n, \alpha)$.

$\phi$ é injetora: Suponha que $f, g \in \operatorname{FP}(\operatorname{Fn}(\omega, J), \kappa)$ são tais que $f \neq g$. Existe $\alpha<\kappa$ tal que $f(\alpha) \neq g(\alpha)$. Se $\operatorname{dom} f(\alpha) \neq \operatorname{dom} g(\alpha)$, sem perda de generalidade existe $n \in \operatorname{dom} f(\alpha) \backslash \operatorname{dom} g(\alpha)$. Segue que $(n, \alpha) \in \operatorname{dom} \phi(f) \backslash \operatorname{dom} \phi(g)$. Se $\operatorname{dom} f(\alpha)=\operatorname{dom} g(\alpha)$, existe $n \in \operatorname{dom} f(\alpha)$ tal que $f(\alpha)(n) \leq g(\alpha)(n)$. Segue que $(n, \alpha) \in \operatorname{dom} \phi(f) \cap \phi(g) \operatorname{mas} \phi(f)(n, \alpha) \neq \phi(g)(n, \alpha)$.

$\phi$ é sobrejetora: tome $p \in Q$. Seja $S=\pi_{2}[\operatorname{supp} p]=\{\alpha<\kappa: \exists n((n, \alpha) \in \operatorname{dom} p)\}$. Seja $f \in \operatorname{FP}(\operatorname{Fn}(\omega, J), \kappa)$ de suporte $S$, onde para $\alpha \in s, \operatorname{dom} f(\alpha)=\{n \in \omega:(n, \alpha) \in \operatorname{dom} p\}$ e, para $n \in \operatorname{dom} f(\alpha)$, seja $f(\alpha)(n)=g(n, \alpha)$. Temos que $\phi(f)=g$.

Finalmente, suponha que $f, g \in \operatorname{FP}(\operatorname{Fn}(\omega, J), \kappa)$ são tais que $\phi(f) \leq \phi(g)$. Devemos ver que $f \leq g$. Fixe $\alpha<\kappa$. Devemos ver que $g(\alpha) \subseteq f(\alpha)$. Fixe $n \in \operatorname{dom} g(\alpha)$. Temos que $(n, \alpha) \in$ $\operatorname{dom} \phi(g) \subseteq \operatorname{dom} \phi(f), \log 0, n \in \operatorname{dom} f(\alpha)$ e $\phi(f)(n, \alpha)=f(\alpha)(n)=g(\alpha)(n)=\phi(g)(n, \alpha)$. Assim, segue a tese.

Com o corolário abaixo, concluímos que Forcing com modelo de Cohen independe de qual exatamente são os conjuntos escolhidos como parâmetros de Fn.

Corolário B.8. Seja $\kappa$ um cardinal infinito, $I, I^{\prime}, J, J^{\prime}$ tais que $|I|=\left|I^{\prime}\right|=\kappa, 2 \leq|J|,\left|J^{\prime}\right| \leq \omega$. Então existem imersões densas de $\operatorname{FP}\left(\omega^{<\omega}, \kappa\right)$ em $\operatorname{Fn}(I, J)$ e $\operatorname{Fn}\left(I^{\prime}, J^{\prime}\right)$, o que implica em essas duas últimas ordens gerarem as mesmas extensões via forcing. 
Demonstração. Basta provar que existe uma imersão densa de $\operatorname{FP}\left(\omega^{<\omega}, \kappa\right)$ em $\operatorname{Fn}(I, J)$. Vimos que existe $i: \omega^{<\omega} \rightarrow \operatorname{Fn}(\omega, J)$ imersão densa. Logo, existe $j: \operatorname{FP}\left(\omega^{<\omega}, \kappa\right) \rightarrow \operatorname{FP}(\operatorname{Fn}(\omega, J), \kappa)$ imersão densa. Mas $\operatorname{FP}(\operatorname{Fn}(\omega, J), \kappa)$ é isomorfo à $\operatorname{Fn}(\omega \times \kappa, J)$, que, por sua vez, é isomorfo à $\operatorname{Fn}(I, J)$ (pois $|I|=|\omega \times \kappa|)$. Assim, segue a tese.

Por fim, vamos enunciar e provar a lei zero-um, mostrando que ela vale para $\operatorname{Fn}(I, J)$ quaisquer que sejam $I, J$ com $I$ infinito.

Definição B.4. Seja $P$ um forcing poset.

Seja $\operatorname{Aut}(P)$ o conjunto dos isomorfismos de $P$ em $P$. Dizemos que $P$ é fracamente homogêneo se, e somente se, para todos $p, q \in P$ existe um automorfismo $i: P \rightarrow P$ tal que $i(p) \not \perp q$.

Proposição B.9. Dados $I, J \operatorname{com} I$ infinito, o forcing poset $P=\operatorname{Fn}(I, J)$ é fracamente homogêneo.

Demonstração. Fixe $q, p \in P$. Seja $u: I \rightarrow I$ bijetora tal que $u[\operatorname{dom} q] \cap \operatorname{dom} p=\emptyset$. Temos que $i: P \rightarrow P$ dado por $i(s)=s \circ u$ é isomorfismo. Note que $\operatorname{dom}(p \circ u)=u^{-1}[\operatorname{dom} p]$ e $u^{-1}[\operatorname{dom} p] \cap$ $\operatorname{dom} q=\emptyset, \operatorname{logo}, i(p), q$ são compatíveis.

Teorema B.10 (Lei Zero-Um). Seja $P$ um forcing poset fracamente homogêneo. Então para toda sentença $\phi$, temos que $1 \Vdash \phi$ ou $1 \Vdash \neg \phi$.

Demonstração. Suponha por absurdo que $\neg(1 \Vdash \phi)$ e $\neg(1 \Vdash \neg \phi)$. Existem $p, q \in P$ tais que $p \Vdash \phi$ e $q \Vdash \neg \phi$. Seja $i: P \rightarrow P$ isomorfismo tal que $i(p)$, $q$ são compatíveis. Temos que $i(p) \Vdash \phi$. Sendo $t \leq i(p), q$ extensão comum, temos que $t \Vdash 0=1$, absurdo.

\section{B.2 O modelo de Cohen e o cardinal $\mathfrak{c}$}

Nesta seção, mostraremos como manipular o cardinal $\mathfrak{c}$ com Forcing de Cohen.

Proposição B.11. Seja $M$ uma interpretação transitiva e enumerável de ZFC. Seja $\kappa \in M$ tal que $(\kappa \text { é um cardinal })^{M}$ e tal que $(\operatorname{cf} \kappa>\omega)^{M}$. Seja $P=\operatorname{Fn}(I, J)$, onde $J \in M,|J|>1$, $(J \text { é enumerável })^{M}$ e $(|I|=\kappa)^{M}$. Seja $G$ um filtro $P$-genérico sobre $M$. Então $(\mathfrak{c} \geq \kappa)^{M[G]}$.

Demonstração. Notemos que $\operatorname{Fn}(I, J)$ é isomorfo, em $M$ à $\operatorname{Fn}(\kappa \times \omega, J)$. Assim, podemos supor, sem perda de generalidade, que $I=\kappa \times \omega$.

Pelo lema anterior, $f: \kappa \times \omega \rightarrow J$ é uma função sobrejetora. Para cada $\alpha \in \kappa$ seja $f_{\alpha}: \omega \rightarrow 2$ dada por $f_{\alpha}(n)=f(\alpha, n)$. Por absolutidade, temos que $\left\{f_{\alpha}: \alpha<\kappa\right\} \in M[G]$. Se mostrarmos que se $\alpha<\beta<\kappa$ então $f_{\alpha} \neq f_{\beta}$, segue que, em $M[G], \kappa=\left|\left\{f_{\alpha}: \alpha<\kappa\right\}\right| \leq\left|J^{\omega}\right|=\mathfrak{c}$. Então provaremos isso. Fixe $j_{0}, j_{1} \in J$ distintos. Suponha que $\alpha<\beta<\kappa$. Seja $D=\{s \in P: \exists n \in$ $\left.\left.\omega\left(\left((n, \alpha), j_{0}\right),\left((n, \beta), j_{1}\right) \in s\right)\right)\right\}$. Se $D$ for denso, segue que $f_{\alpha}(n) \neq f_{\beta}(n)$ para algum $n$, e, assim, segue a tese. Então basta ver que $D$ é denso. Dado $s \in P$, seja $n \in \omega$ tal que $n \neq m$ para todo $m$ tal que existe $\delta$ tal que $(\delta, m) \notin \operatorname{dom} s$, o que é possível pois $s$ é finito (i.e, tome $n \in \omega \backslash \pi_{2}[\operatorname{dom} s]$ ). Temos que $s \cup\left\{\left((\alpha, n), j_{0}\right),\left((\beta, n), j_{1}\right)\right\} \in D$ é uma extensão de $s$.

Notemos que com o que temos em mãos até agora, já podemos afirmar que a consistência de ZFC implica na consistência de ZFC $+\neg \mathrm{CH}$. Veremos a seguir que $(\kappa=\mathfrak{c})^{M[G]}$ desde que $M$ satisfaça $\mathrm{GCH}$. Podemos atingir isso de diversas formas. Mencionaremos duas formas: Trabalhando na teoria $\mathrm{ZFC}+\mathrm{V}=\mathrm{L}+\mathrm{M}$, em que $M$ é um símbolo de constante transitivo e enumerável que satisfaz cada axioma de $\mathrm{ZFC}+\mathrm{V}=\mathrm{L}$, ou podemos utilizar a teoria $\mathrm{ZFCM}$ ', em que M é um símbolo de constante transitivo e enumerável que satisfaz os axiomas de $\mathrm{ZFC}+\mathrm{V}=\mathrm{L}$, mas em que $\mathrm{V}=\mathrm{L}$ não é um axioma (diferentemente da teoria $\mathrm{ZFC}+\mathrm{V}=\mathrm{L}+\mathrm{M}$ ). Essa teoria também é conservativa sobre $\mathrm{ZFC}$ com uma demonstração análoga à da teoria ZFCM, pois é possível demonstrar que para qualquer quantidade finita axiomas de $\mathrm{ZFC}+\mathrm{V}=\mathrm{L}$, $\mathrm{ZFC}$ prova que existe um conjunto transitivo e enumerável que satisfaz esses axiomas. 
Proposição B.12. Suponha que $M$ é uma interpretação transitiva e enumerável de ZFC, $P \in M$ um forcing poset com c.c.c., $\kappa, \lambda \in M$ um cardinal infinito de $M$, e que em $M$, vale que $|P|=\kappa$. Seja $\theta=\left(\kappa^{\lambda}\right)^{M}$. Seja $G$ um filtro $P$-genérico sobre $M$. Então:

1. Em $M$, existem no máximo $k^{\lambda}$ nice names para subconjuntos de $\check{\lambda}$.

2. Em $M[G]$, vale que $2^{\lambda} \leq \theta$.

Demonstração. Trabalhemos em $M$. A ideia aqui é contar os nice names de subconjuntos de $\check{\lambda}$. Seja $\mathcal{A}$ a coleção de todas as anticadeias de $P$, e $\mathcal{F}=\mathcal{A}^{\lambda}$. Seja $F$ função de domínio $\mathcal{F}$ dada por $F(f)=\bigcup_{\alpha \in \lambda}\{\check{\alpha}\} \times f(\alpha)$. Temos que a imagem de $F$ é o conjunto de todos os nice names de subconjuntos de $\lambda$. Seja $\mathcal{B}$ este conjunto. Temos, portanto, que $|\mathcal{B}| \leq|\mathcal{F}|$. Temos que todo elemento de $\mathcal{A}$ é um subconjunto enumerável de $P$, logo, $|\mathcal{F}|=\left|A^{\lambda}\right|=|A|^{\lambda} \leq\left|[P]^{<\omega}\right|^{\lambda}=\left(\kappa^{\omega}\right)^{\lambda}=\kappa^{\lambda}=\theta$.

Paremos de trabalhar em $M$. Vimos que existe $f \in M$, tal que $f: \mathcal{B} \rightarrow \theta$ injetora. Seja $g: \mathcal{B} \rightarrow$ $\mathscr{P}(\lambda)$ dada por $g(\tau)=\tau_{G}$. Por absolutidade, $g \in M[G]$. Assim, temos que $g: \mathcal{B} \rightarrow(\mathscr{P}(\lambda))^{M[G]}$. Esta função é sobrejetora, pois seja $X \in(\mathscr{P}(\lambda))^{M[G]}$. Seja $\rho$ um $P$-nome em $M$ para $X$. Existe $p \in G$ tal que $p \Vdash \rho \subseteq \check{\lambda}$. Existe $\tau \in M^{P}$ tal que $\tau$ é um nice name para um subconjunto de $\check{\lambda}$ e $1 \Vdash(\rho \subseteq \check{\lambda} \rightarrow \tau=\rho)$. Em particular, $p \Vdash(\rho \subseteq \check{\lambda} \rightarrow \tau=\rho)$. Mas então, $p \Vdash \tau=\rho$. Como $p \in G$, segue que $g(\tau)=\tau_{G}=\rho_{G}=X$. Assim, como $P$ preserva cardinais e $f, g \in M$, segue que, em $M[G]$ :

$$
2^{\lambda}=|\mathscr{P}(\lambda)| \leq|\mathcal{B}| \leq|\theta|=\theta
$$

Corolário B.13. Suponha que $M$ é uma interpretação transitiva e enumerável de ZFC, $\kappa, \lambda \in M$ um cardinal infinito de $M, P=\operatorname{Fn}(I, J)$, onde $|J| \geq 2, J \in M$ e ( $J$ é enumerável, $|I|=\kappa)^{M}$. Seja $G$ um filtro $P$-genérico sobre $M$. Então:

i) Se $\left(\kappa=\kappa^{\omega}\right)^{M}$ então $(\mathfrak{c}=\kappa)^{M[G]}$.

ii) Se $M$ satisfaz GCH e se $(\operatorname{cf} \kappa>\omega)^{M}$ então $(\mathfrak{c}=\kappa)^{M[G]}$.

Demonstração. Pela Proposição B.11, temos que $\left(2^{\omega} \geq \kappa\right)^{M[G]}$. Assim, basta mostrar que ( $2^{\omega} \leq$ $\kappa)^{M[G]}$. Se vale i), isso segue diretamente da proposição anterior. Se valem as hipóteses de ii), então as hipóteses de i) decorrem de iii) da Proposição 1.11.

\section{B.3 O modelo de Cohen e o cardinal $\mathfrak{b}$}

Nesta seção, veremos que, no modelo de Cohen, $\mathfrak{b}=\omega_{1}$.

Lema B.14. Seja $M$ uma interpretação transitiva e enumerável de ZFC. Seja $Q \in M$ uma noção de forcing enumerável em $M$, e seja $G$ um filtro $\mathbb{Q}$-genérico sobre $M$. Então $\omega^{\omega} \cap M$ é ilimitado em $M[G]$.

Demonstração. Suponha que não. Existe $h \in \omega^{\omega} \cap M[G]$ tal que $f \leq^{*} h$ para todo $f \in \omega^{\omega} \cap M$. Seja $\tau \in M^{Q}$ tal que $\tau_{G}=h$. Seja $W=\omega^{\omega} \cap M$. Fixe $p \in G$ tal que $p \Vdash^{*} \tau: \check{\omega} \rightarrow \check{\omega} \wedge \forall x \in \check{W}\left[x \leq^{*} \tau\right]$. Trabalhando em $M$, seja $\{q \in Q: q \leq p\}=\left\{r_{j}: j \in \omega\right\}$. Para cada $n$, seja $E_{n}=\{l \in \omega: \exists j<$ $\left.n\left[r_{j} \Vdash^{*} \check{l} \leq \tau(n)\right]\right\}$. Como para cada $j<n$ existe no máximo um $l$ tal que $r_{j} \Vdash^{*} \check{l} \leq \tau(n)$, segue que para todo $n \in \omega, E_{n}$ é finito. Seja $f(n)=\max \left(E_{n}\right)+1$, definindo-se arbitrariamente $\max \emptyset=0$. Temos que $f \in \omega^{\omega}$.

Voltemos para fora de $M$. Como trabalhamos em $M$, segue que $f \in W$. Segue que $p \Vdash \exists m \in$ $\check{\omega} \forall n \geq m[\check{f}(n) \leq \tau(n)]$. Seja $q \leq p$ e $m \in \omega$ tal que $p \Vdash \forall n \geq \check{m}[\check{f}(n) \leq \tau(n)]$. Existe $j$ tal que $q=r_{j}$. Fixe $n$ tal que $n>m, j$. Seja $l=f(n)$. Temos que $r_{j} \Vdash \breve{l} \leq \tau(n)$. Logo, pela definição de $f$, temos que $f(n)>l$ o que é absurdo. 
Lema B.15. Seja, $I, J$ quaisquer e $K \subseteq I$. Seja $i: \operatorname{Fn}(K, J) \rightarrow \operatorname{Fn}(I, J)$ a inclusão. Então $i$ é uma imersão completa.

Demonstração. É claro que $i$ preserva a ordem, assim, se $s, t \operatorname{Fn}(K, J)$ são compatíveis, também o são em $\operatorname{Fn}(I, J)$. Reciprocamente, se $s, t$ são compatíveis em $\operatorname{Fn}(K, J)$, existe $p \in \operatorname{Fn}(I, J)$ tal que $p \subseteq s, t$. Logo, $\operatorname{dom} p \subseteq \operatorname{dom} s \subseteq K$, e, assim, $s, t$ são compatíveis em $\operatorname{Fn}(K, J)$.

Por fim, seja $q \in \operatorname{Fn}(I, J)$. Devemos encontrar uma redução de $q$ em $\operatorname{Fn}(K, J)$, ou seja, devemos encontrar $p \in \operatorname{Fn}(K, J)$ tal que para todo $p^{\prime} \in P$, se $p^{\prime} \leq p$, então $p^{\prime}$ e $q$ são compatíveis em $\operatorname{Fn}(I, J)$. Afirmo que $p=q \mid K$ faz esse papel. Com efeito, se $p^{\prime} \leq p$ então $\operatorname{dom} p^{\prime} \cap \operatorname{dom} q=\operatorname{dom} p^{\prime} \cap \operatorname{dom} p=$ $\operatorname{dom} p$, e $q, p^{\prime}$ coincidem em $\operatorname{dom} p$ (ambas, restritas, são $p$ ). Assim, segue a tese.

Proposição B.16. Seja $M$ uma interpretação transitiva e enumerável de ZFC. Seja $P=\operatorname{Fn}(I, J)$, onde $I, J \in M$ e $(|J| \leq \omega)^{M}$. Seja $G$ um filtro $P$-genérico sobre $M$. Então não existe $h \in \omega^{\omega} \cap M[G]$ tal que $f \leq^{*} h$ para todo $f \in \omega^{\omega} \cap M$. Em particular, se $M$ satisfaz a hipótese do contínuo, então, em $M[G], \mathfrak{b}=\omega_{1}$.

Demonstração. Suponha por absurdo que exista uma função $h$ como no enunciado e seja $\tau \in M^{P}$ um nice name para um subconjunto de $(\omega \check{\times} \omega)$ tal que $\tau_{G}=h$. Trabalhemos em $M$. Seja $S$ a união enumerável das anticadeias enumeráveis (pois $P$ tem c.c.c.) de $\tau$. Temos que $S$ é enumerável. Temos que $K=\bigcup\{\operatorname{dom} q: q \in S\} \subseteq I$ é enumerável. Seja $Q=\operatorname{Fn}(K, J)$. Se $H=G \cap Q$, temos que $H$ é $Q$-genérico sobre $M$ e $M \subseteq M[H] \subseteq M[G]$. Temos que $h=\tau_{G}=\tau_{H} \in M[H]$ e $f \leq^{*} h$ para todo $f \in \omega^{\omega} \cap M$. Mas isso contradiz o lema anterior.

A segunda afirmação decorre do fato de que, em $M[G], W=\omega^{\omega} \cap M$ é uma família ilimitada de cardinalidade $\omega_{1}$ (pela preservação de $\omega_{1}$ ).

\section{B.4 O Modelo de Cohen e o cardinal a}

Nesta seção, veremos que, no modelo de Cohen, $\mathfrak{a}=\omega_{1}$.

Proposição B.17. Seja $M$ uma interpretação transitiva e enumerável de $\mathrm{ZFC}+\mathrm{CH}$. Existe $\mathcal{A} \in M$ MAD family de $M$ tal que para todos $I, J$ em $M$ tais que $I$ é infinito e $(2 \leq|J| \leq \omega)^{M}, \mathcal{A}$ é $\operatorname{Fn}(I, J)$-indestrutível. Em particular, se $G$ é $\operatorname{Fn}(I, J)$-genérico sobre $M$, segue que $\left(\mathfrak{a}=\omega_{1}\right)^{M[G]}$.

Demonstração. Trabalhemos em $M$. Seja $P=\omega^{<\omega}$. Note que, pela Proposição B.12, existem no máximo $\omega^{\omega}=\omega_{1}$ nice $P$-names para subconjuntos de $\omega$. Seja $\left\{\left(\tau_{\xi}, p_{\xi}\right): \omega \leq \xi<\omega_{1}\right\}$ a coleção de todos os pares $(\tau, p)$ tais que $\tau$ é um nice $P$-name para um subconjunto de $\check{\omega}, p \in P$ e $p \Vdash|\tau|=\omega$.

Seja $\left\{A_{n}: n \in \omega\right\}$ uma partição infinita de $\omega$. Suponha definido $A_{\xi}$ para todo $\xi<\alpha$ para algum $\alpha$ tal que $\omega \leq \alpha<\omega_{1}$. Escolhe-se $A_{\alpha}$ tal que:

a) $\left|A_{\alpha} \cap A_{\xi}\right|<\check{\omega}$ para todo $\xi<\alpha$.

b) Se $\forall \xi<\alpha\left(p_{\alpha} \Vdash^{*}\left|\tau_{\alpha} \cap \check{A}_{\xi}\right|<\omega\right)$, então $\forall m \in \omega \forall q \leq p_{\alpha} \exists r \leq q \exists n>m\left(n \in A_{\alpha} \wedge r \Vdash^{*} \check{n} \in \tau_{\alpha}\right)$.

Isso pode ser feito: Se a hipótese em b) é falsa, então basta tomar $A_{\alpha} \in[\omega]^{\omega}$ que mostra que $\left\{A_{\xi}: \xi<\alpha\right\}$ não é MAD (pois é enumerável). Caso seja satisfeita, seja $\left\{B_{i}: i<\omega\right\}=\left\{A_{\xi}: \xi<\alpha\right\}$ e $\left\{\left(n_{i}, q_{i}\right): i<\omega\right\}=\omega \times\left\{q \in P: q \leq p_{\alpha}\right\}$. Para cada $i$, temos que $q_{i} \Vdash\left(\left|\tau_{\alpha} \backslash\left(\check{B}_{0} \cup \cdots \cup \check{B}_{i}\right)\right|=\check{\omega}\right)$. Assim, existem $r_{i} \leq q_{i}$ e $m_{i} \geq n_{i}$ tais que $m_{i} \notin B_{0} \cup \cdots \cup B_{i}$ e $r_{i} \Vdash \check{m}_{i} \in \tau_{\alpha}$. Seja $A_{\alpha}=\left\{m_{i}: i \in \omega\right\}$ e a recursão está completa.

Seja $G$ um filtro $P$-genérico sobre $M$. Afirmo que $\mathcal{A}$ é MAD em $M[G]$ (note que isso implica que $\mathcal{A}$ é MAD em $M$, e que é $P$-indestrutível). Pois suponha que não. Existe $X \in M$ com $X \subseteq \omega$ infinito tal que $\left|X \cap A_{\alpha}\right|<\omega$ para todo $\alpha<\omega_{1}^{M}$. Seja $\tau \in M$ um $P$-nice name tal que $\tau_{G}=X$. Existe $p \in P$ tal que $p \Vdash(|\tau|=\omega$ e $\forall A \in \check{\mathcal{A}}(|A \cap \tau|<\check{\omega}))$. Existe $\alpha<\omega_{1}^{M}$ tal que $(\tau, p)=\left(\tau_{\alpha}, p_{\alpha}\right)$. Note que hipótese de b) vale para o passo $\alpha$ da recursão. Temos que $p_{\alpha} \Vdash^{*}\left|\tau_{\alpha} \cap \check{A}_{\alpha}\right|<\omega$, logo, existe $m \in \omega$ e $q \leq p_{\alpha}$ tal que $q \Vdash \tau_{\alpha} \cap \check{A}_{\alpha} \subseteq \check{m}$. Mas existe $\leq q$ e $n>m$ tal que $n \in A_{\alpha}$ e 
$r \Vdash \check{n} \in \tau_{\alpha}$, o que é absurdo.

Agora seja $Q=\operatorname{Fn}(I, J)$ como no enunciado. Suponha por absurdo que exista $G$ filtro $P$-genérico sobre $M$ tal que $\mathcal{A}$ não é MAD em $M[G]$. Existe $X \in M[G]$ tal que $X \subseteq \omega$ é infinito e $X \cap A$ é finito para todo $A \in \mathcal{A}$. Seja $\tau$ um nice name para um subconjunto de $\check{\omega}$ tal que $\tau_{G}=X$. Novamente, as união das anticadeias (enumeráveis) de $G$ é um conjunto enumerável $K$. Seja $Q^{\prime}=\operatorname{Fn}(K, J)$ Notemos que $\tau$ é um nice $Q^{\prime}$-name para um subconjunto de $\check{\omega}$. Seja $H=G \cap Q^{\prime}$. Temos que $H$ é $Q^{\prime}$-genérico sobre $M$ e $\tau_{h}=\tau_{G}$, então $\mathcal{A}$ não é MAD em $M[H]$. Existe $i: P \rightarrow Q^{\prime}$ imersão densa. Segue que $i^{-1}[H]$ é $P$-genérico sobre $M$ e $M\left[i^{-1}[H]\right]=M[H]$. Mas isso é absurdo pois $\mathcal{A}$ é MAD em $M\left[i^{-1}[H]\right]$.

\section{B.5 O Modelo de Cohen e o cardinal d}

Nesta seção, veremos que, no modelo de Cohen, $\mathfrak{d}=\omega_{1}$.

Lema B.18. Seja $M$ uma interpretação transitiva e enumerável de ZFC. Sejam $I, W \in M$ e $P=\operatorname{Fn}(I, J), Q=\operatorname{Fn}(W, J)$ e $R=\operatorname{Fn}(I \backslash W, J)$. Seja $K$ um filtro $P$-genérico sobre $M$. Seja $G=K \cap Q$ e $H=K \cap R$. Então $G$ é $\operatorname{Fn}(W, J)$-genérciso sobre $M, H$ é $\operatorname{Fn}(I \backslash W, J)$-genérico sobre $M[G]$ e $M[K]=M[G][H]$.

Demonstração. Seja $\phi: Q \times R \rightarrow P$ dada por $\phi(p, q)=p \cup q$. Verifica-se que $\phi$ é um isomorfismo. Seja $\tilde{K}=\phi^{-1}[K]$. Temos que $\tilde{K}$ é $R \times S$-genérico sobre $M$ e $M[K]=M[\tilde{K}]$. Notemos que $\tilde{K}=G \times H$. Assim, segue que $G$ é $Q$-genérico sobre $M, H$ é $K$-genérico sobre $M[G]$ e $M[K]=M[\tilde{K}]=$ $M[G][H]$.

Lema B.19. Seja $P=\operatorname{Fn}(I, J)$ e $M$ uma interpretação transitiva e enumerável de ZFC tal que $I, J \in M$ e $(2 \leq|J| \leq \omega,|I|=\omega)^{M}$. Seja $X=\omega^{\omega} \cap M$. Temos que $\left.1 \Vdash_{P} \exists f \in \omega^{\omega} \forall h \in \check{X} \neg\left(f \leq^{*}\right)\right\}$.

Demonstração. Como forcing com $\operatorname{Fn}(I, J)$ e $\operatorname{Fn}(\omega, \omega)$ são equivalentes, podemos supor que $I=$ $J=\omega$. Seja $G$ uma filtro $P$-genérico sobre $M$ e seja $f=\bigcup G: \omega \rightarrow \omega$. Afirmo que se $h \in K$ então $\neg\left(f \leq^{*} h\right)$. Para isso, fixe $h$. Para cada $m \in \omega$, temos que, por absolutidade, $D_{m}=\{p \in$ $P: \exists n \in \operatorname{dom} p(n>m \wedge p(n)>h(n))\} \in M$. $D_{m}$ é denso em $P$, pois dado $s \in P$, sendo $n>m$, $m \notin \operatorname{dom} p$, temos que $p \cup\{(n, h(n)+1)\} \in D_{m}$. Agora suponha por absurdo que $f \leq{ }^{*} h$. Existe $m$ tal que se $n>m$ então $f(n) \leq h(n)$. Mas, como existe $s \in D_{m} \cap G$, segue que existe $n>m$ tal que $f(n)>h(n)$, o que é absurdo.

Proposição B.20. Seja $M$ uma interpretação transitiva e enumerável de ZFC. Seja $\kappa$ um cardinal de $M$ e seja $P=\operatorname{Fn}(I, J)$, onde $I, J \in M$ e $(|I|=\kappa, 2 \leq|J| \leq \omega)^{M}$. Seja $K$ um filtro $P$-genérico sobre $M$. Então $(\mathfrak{d} \geq \kappa)^{M[K]}$.

Demonstração. Suponha por absurdo que o resultado é falso. Existe, em $M[K]$, um cardinal $\theta<\kappa$ e uma função $h: \theta \times \omega \rightarrow \omega$ tal que $\left\{h_{\alpha}: \alpha<\theta\right\}$ é uma família dominante, onde $h_{\alpha}(n)=h(\alpha, n)$ para todos $\alpha<\theta, n \in \omega$. Como toda família dominante é ilimitada e não existem famílias ilimitadas enumeráveis, temos que $\theta \geq \omega_{1}^{M}=\omega_{1}^{M[K]}$. Seja $\tau$ um nice name para um subconjunto de $(\theta \times \check{\omega}) \times \omega$ tal que $\tau_{K}=h$.

Trabalhemos em $M$. Seja $U \subseteq \kappa$ tal que $|U| \leq \theta$ união de todos os domínios dos elementos das anticadeias (enumeráveis) de $\tau$. Tomemos $W$ tal que $U \subseteq W \subseteq \kappa$ e $|\kappa \backslash W|=\omega$. Temos que $\tau$ é um $\operatorname{Fn}(W, J)$-nome. Seja $Q=\operatorname{Fn}(W, J), R=\operatorname{Fn}(\kappa \backslash W, J), G=Q \cap K, H=Q \cap K$. Temos que $G$ é $Q$-genérico sobre $M, H$ é $R$-genérico sobre $M[G]$ e $M[K]=M[G][H]$. Temos que $h=\tau_{H} \in M[H]$. Pelo lema anterior, existe $f \in M[G][H]$ tal que $\forall \alpha<\theta \neg\left(f \leq^{*} h_{\alpha}\right)$, o que é absurdo.

Assim, neste apêndice, utilizando o fato de que $\omega_{1} \leq \mathfrak{h} \leq \mathfrak{b}$, chegamos ao seguinte corolário: 
Corolário B.21. Seja $M$ uma interpretação transitiva e enumerável de ZFC + GCH. Suponha que, em $M, \kappa$ é um cardinal infinito e cf $\kappa>\omega$. Sejam $I, J \in M$ tais que $(|I|=\kappa, 2 \leq|J| \leq \omega$. Seja $G$ um filtro $\operatorname{Fn}(I, J)$ genérico sobre $M$. Então $\left(\omega_{1}=\mathfrak{h}=\mathfrak{a}=\mathfrak{b} \leq \kappa=\mathfrak{d}=\mathfrak{c}\right)^{M[G]}$. Em particular:

$$
\operatorname{Con}(\mathrm{ZFC}) \rightarrow \operatorname{Con}\left(\mathrm{ZFC}+\left(\omega_{1}=\mathfrak{h}=\mathfrak{a}=\mathfrak{b}<\mathfrak{d}=\mathfrak{c}\right)\right)
$$


APÊNDICE B 


\section{Referências Bibliográficas}

[Bel81] M.G. Bell. On combinatorial principle p(c). Fund. Math., 114(2):149-157, 1981. 103

[Bla10] Andreas Blass. Combinatorial Cardinal Characteristics of the Continuum, páginas 395489. Springer Netherlands, 2010. 1, 30, 91

[CN74] W.W. Comfort e S. Negrepontis. The Theory of Ultrafilters. Grundlehren der mathematischen Wissenschaften in Einzeldarstellungen mit besonderer Berücksichtigung der Anwendungsgebiete. Springer-Verlag, 1974. 16

[Dow95] Alan Dow. More set-theory for topologists. Topology and its Applications, 64(3):243 300, 1995. 1

[DS06] Alan Dow e Petr Simon. Spaces of continuous functions over a $\psi$-space. Topology and its Applications, 153(13):2260 - 2271, 2006. 1, 45

[Fra65] S. P. Franklin. Spaces in which sequences suffice. Fund. Math., 57:106-115, 1965. 1, 55

[Fra67] S. P. Franklin. Spaces in which sequences suffice ii. Fund. Math., 61:51-56, 1967. 1, 55

[Gin75] J. Ginsburg. Some results on the countable compactness and pseudocompactness of hyperspaces. Canad. J. Math., 27(6):1392-1399, 1975. 83, 86

[HHR07] M. Hrušák, F. Hernández e I. Ruiz. Pseudocompactness of hyperspaces. Topology and it's Applications, 154:3048-3055, 2007. 1, 34, 83, 88, 98

[Hru01] Michael Hrušák. Mad families and the rationals. Comment. Math. Univ. Carolin., 42:345$352,2001.1,73,74$

[Hru14] Michael Hrušák. Almost Disjoint Families and Topology, páginas 601-638. Atlantis Press, Paris, 2014. 1, 27, 96

[I.B80] A. I. A. I.Bashkirov. On continuous maps of isbell spaces and strong 0-dimensionality. Bull. Acad. Polon. Sci. Sér. Sci. Math., 27:605-611, 1980. 63

[Kun80] K. Kunen. Set Theory: An Introduction to Independence Proofs. Mathematical Programming Study. North-Holland Publishing Company, 1980. 1, 21, 27, 74

[Kun11] K. Kunen. Set Theory. Studies in logic. College Publications, 2011. 1, 2, 29, 74, 103

[Mat77] A.R.D. Mathias. Happy families. Annals of Mathematical Logic, 12(1):59 - 111, 1977. 1, 40

[Mic51] Ernest Michael. Topologies on spaces of subsets. Trans. Amer. Math. Soc, 71:151-182, 1951. 1,83

[Mro70] S. Mrowka. Some comments on the author's example of a non-R-compact space. Bull. Acad. Polon. Sci. S?r. Sci. Math. Astronom. Phys., 18:443-448, 1970. 1, 27, 66 
[Mro77] S. Mrowka. Some set-theoretic constructions in topology. Fundamenta Mathematicae, 94(2):83-92, 1977. 1, 35, 66, 68, 69

[MS16] M. Malliaris e S. Shelah. Cofinality spectrum theorems in model theory, set theory, and general topology. J. Amer. Math. Soc., 29:237-297, 2016. 91

[Sim80] Petr Simon. A compact fréchet space whose square is not fréchet. Commentationes Mathematicae Universitatis Carolinae, 021(4):749-753, 1980. 1

[Sim03] Petr Simon. d-3 - Fréchet and Sequential Spaces. Em Encyclopedia of General Topology, páginas 162 - 164. Elsevier, Amsterdam, 2003. 55

[Ter80] Jun Terasawa. Spaces $N \cup R$ and their dimensions. Topology and its Applications, 11(1):93 $-102,1980.1,70$

[Wal75] R.C. Walker. The Stone-Cech Compactification. Ergebnisse der Mathematik und ihrer Grenzgebiete. 2. Folge. Springer, 1975. 1, 12, 57

[Wil04] S. Willard. General Topology. Addison-Wesley series in mathematics. Dover Publications, 2004. 12,56 